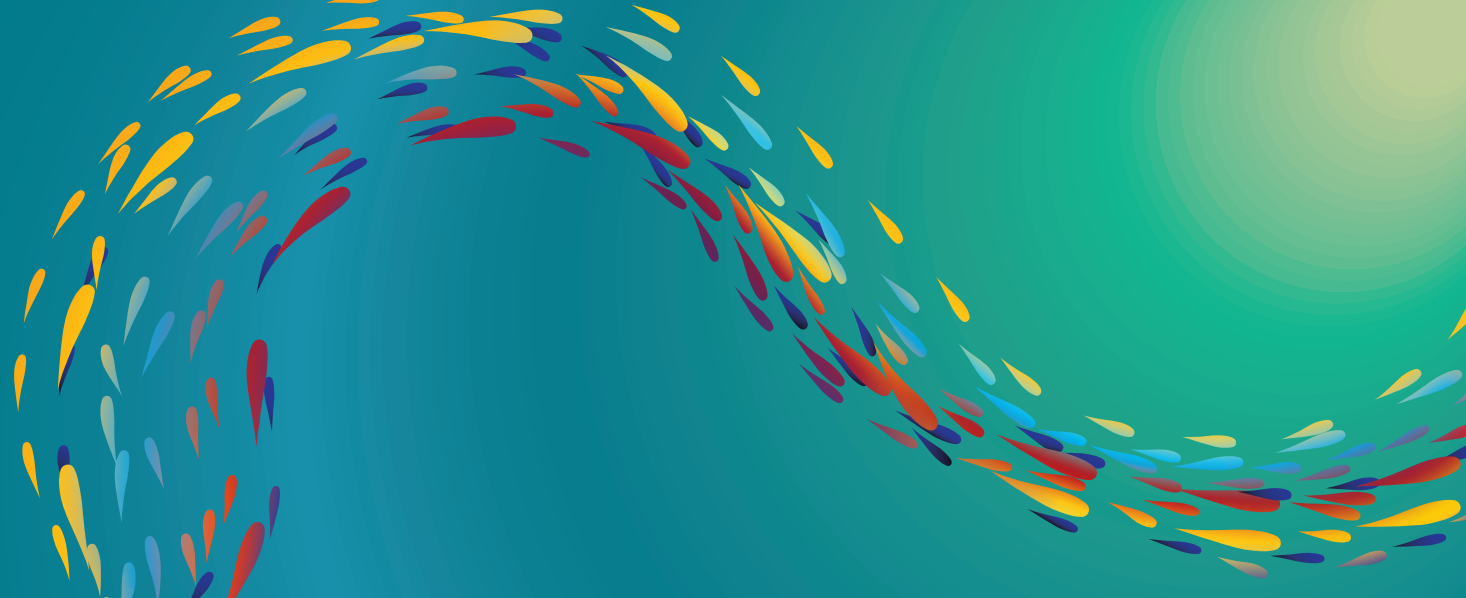

\title{
Polarized innate immunity: conservation of macrophage polarization in carp
}




\section{Propositions}

1. Carp macrophages have the ability to steer and polarize innate immune responses. (this thesis)

2. Polarization states of macrophages should be determined using a set of markers rather than a single one. (this thesis)

3. If mastered by many, computer programming can take life sciences to the next level.

4. Empirically derived food supplements without ample underlying evidence for safety and efficacy should not lightheadedly be approved for human consumption.

5. To help bridge the gap between science and society, scientists should get and take the time to inform the general public about their research results.

6. Obliged quota aimed at increasing the proportion of women in science will reduce the acceptance and respect towards women.

7. In line with the protection of laboratory animals by the 'five freedoms', a proof of proficiency should be implemented before buying or adopting domestic animals.

Propositions belonging to the thesis, entitled

Polarized innate immunity: conservation of macrophage polarization in carp

Annelieke Simone Wentzel

Wageningen, 22 June 2020 



\section{Polarized innate immunity: conservation of macrophage polarization in carp}




\section{Thesis Committee}

\section{Promotors}

Prof. Dr G. F. Wiegertjes

Professor of Aquaculture and Fisheries

Wageningen University \& Research

Dr M. Forlenza

Associate professor, Cell biology and Immunology Group

Wageningen University \& Research

\section{Other members}

Prof. Dr A. H. Kersten, Wageningen University \& Research

Dr H. J. W. C. Megens, Wageningen University \& Research

Dr A. Lammers, Wageningen University \& Research

Dr M. E. Nguyen-Chi, Université Montpellier, France

This research was conducted under the auspices of the Graduate School Wageningen Institute of Animal Sciences. 


\title{
Polarized innate immunity: conservation of macrophage polarization in carp
}

\author{
Annelieke S. Wentzel
}

\section{Thesis}

Submitted in fulfilment of the requirements for the degree of doctor at Wageningen University

by the authority of the Rector Magnificus,

Prof. Dr A. P. J. Mol,

in the presence of the

Thesis Committee appointed by the Academic Board

to be defended in public

on Monday 22 June 2020

at 4 p.m. in the Aula 


\section{A.S. Wentzel}

Polarized innate immunity: conservation of macrophage polarization in carp, 236 pages.

PhD thesis, Wageningen University, Wageningen, the Netherlands (2020)

With references, with summaries in English and Dutch

ISBN: 978-94-6395-378-8

DOI: $10.18174 / 519758$ 




\section{Table of contents}

Chapter $1 \quad$ General introduction

Chapter 2 Polarization of immune responses in fish: the 27

'macrophages first' point of view

Chapter 3 Transcriptome sequencing supports a striking

conservation of macrophage polarization in fish

Chapter 4 Paralogs of common carp granulocyte colonystimulating factor (G-CSF) have different functions regarding development, trafficking and activation of neutrophils

Chapter 5 Fish macrophages show distinct metabolic signatures upon polarization

Chapter 6 General discussion

Summaries

Summary (English)

Samenvatting (Nederlands)

About the author

About the author

List of publications

Overview of completed training activities

Acknowledgements 

1

General introduction

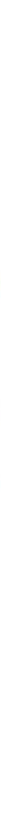




\section{Aquaculture of common carp}

In the past decades, the aquaculture industry has undergone rapid growth driven by the increasing need to feed a growing world population. Cultured fish production has increased almost fourfold over the last twenty years and is expected to increase even further [1, 2] (Figure 1.1A). These increases in production drive a strong demand for efficient and sustainable culture methods that also ensure good quality of life for the animals and without straining the environment. One of the most important issues in aquaculture is the number of infectious diseases and thus threat to the health and welfare of fish living in dense populations and stressful conditions. Particularly in aquaculture, poor knowledge of microbial diversity in farm systems leads to sudden outbreaks of previously unknown pathogens [3]. To prevent and combat infectious disease pressure, livestock and aquaculture production systems have sometimes turned to the use of antibiotics. However, the release of antibiotics in open water increases the risk of developing antibiotic resistance of microorganisms and leads to environmental pollution. Vaccines can be a preventive rather than curative measure contributing to the combat, but relatively few protective vaccines are currently available for fish and their development can be challenging and costly. Especially for fish species of relatively low economic value, the drive to develop costly vaccines can be minimal. In aquaculture, this results in a further need to explore health-promoting preventive approaches based on immunomodulation in the broadest sense. To investigate immunomodulating approaches both fundamental and applied knowledge of immune responses of cultured fish is of crucial importance.

Teleost fish are among the first vertebrates that have developed adaptive immunity [4], which likely took shape due to 2 rounds of whole genome duplications at the start of the vertebrate lineage and the emerging recombination-activating-gene (RAG) transposon [5] (Figure 1.1B). By some, they are considered an ideal model to study the underpinnings of immune systems because of their phylogenetic position [6, 7] . Common carp (Cyprinus carpio) are not only among the oldest cultured fish species [8], it is also the fourth most cultured fish species worldwide (over 4 million tons in 2017) comprising approximately $5 \%$ of all and $10 \%$ of fresh water aquaculture [2] (Figure 1.1A). Moreover, their omnivorous nature means they are a more sustainable option than carnivorous species because they rely less on fish-based diets. Furthermore, carp are important representatives of the family of cyprinid fishes and are therefore an interesting species to study the development 
of their immune system. The combined advantages of i) their economic importance for aquaculture and ii) their evolutionary position and close genetic relation to the well-described animal model species zebrafish (Danio rerio) (Figure 1.1B) makes carp an interesting species to study. For these reasons, together with the access to its genome, is common carp our experimental species of choice.

A

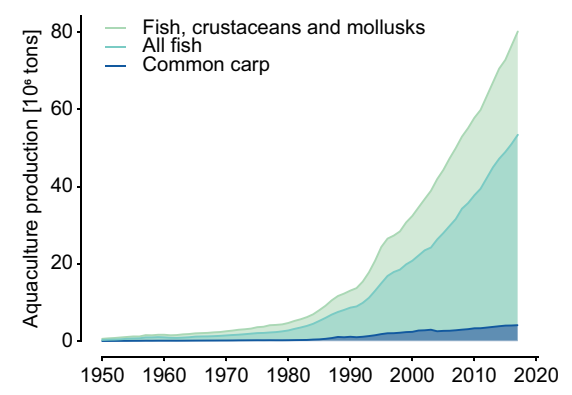

B

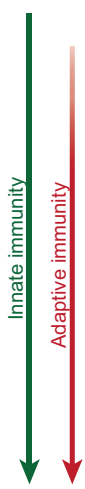

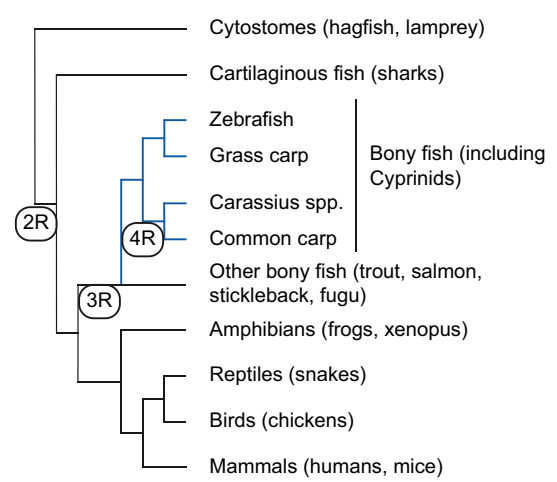

Figure 1.1 Importance of common carp in aquaculture and its place in evolution. A) Aquaculture production growth from 1950 until 2016 in million tons. Lines indicate total aquaculture production (all fish, crustaceans, mollusks and excluding plants), production of all fish species and production of common carp specifically ${ }^{1}$. B) Schematic overview of phylogenetic relationships of carp and other (but not all) key vertebrate species. Arrows indicate the presence of innate immunity (green) and the development of adaptive immunity (red). Rounds (R) of whole genome duplication events are indicated as $2 \mathrm{R}$, 3R (teleost specific duplication) and $4 \mathrm{R}$ (duplication in goldfish and carp). Other lineages that underwent separate $4 \mathrm{R}$ duplications (for example salmon and trout) are not indicated. Figure based on $[5,9-11]$

\section{Macrophage recognition of pathogens and activation}

Cold-blooded species rely more heavily on innate immunity, particularly at lower temperatures when adaptive immune responses are delayed or even impaired [12, 13]. Classically, innate immunity has been viewed to be rapid but with limited specificity and diversity, which has caused it to stay in the shadow of the rapidly evolving field of adaptive immune responses [14]. However, immunologists have realized over the past decades that innate immune responses do not only deal with the bulk of the pathogen load, but they are also much more tailored towards the specific pathogen than originally presumed (Medzhitov and Janeway, 2000). In mammals, but also in fish, innate immunity may even display a form of memory

1 Statistics derived from FAO database on 2020-03-02 http://www.fao.org/fishery/statistics/global-aquaculture-production/query/en 
termed 'trained immunity' [16-18]. Therefore, innate immunity plays a prominent role in host defense of mammals, and even more so of fish.

Macrophages are essential for the development of efficient innate immune responses and involved in every stage of infection. For example, macrophages phagocytose pathogens, produce pro-inflammatory cytokines, produce antimicrobial nitric oxide (NO) and other reactive oxygen radicals (ROS) in the inflammatory phase of infections. The vast amount of pro- and anti-inflammatory cytokines and chemokines produced can recruit and activate other immune cells such as neutrophils, which are particularly important in the inflammatory phase of infection and are studied in this thesis (chapter 4). Macrophages also present antigens to activate adaptive immune responses and clean up dead cells and debris at later stages of infection. Furthermore, macrophages play a crucial role in tissue remodeling through production of proline and polyamines and regulate immune responses through the production of anti-inflammatory cytokines. This extreme plasticity of macrophages allows these cells to perform seemingly opposite functions, which are triggered by signals in their direct microenvironment such as the presence of micro-organisms and cytokines produced by other cells.

Pattern recognition receptors (PRRs) are the main mechanism responsible for the recognition of micro-organisms through particular structures referred to as pathogen associated molecular patterns (PAMPs). Pattern recognition receptors can be membrane-bound or cytosolic receptors and recognize PAMPs such as lipopolysaccharide (LPS) from gram-negative bacteria, viral DNA or RNA and glycans from the cell wall of fungi and bacteria [19] but also damage associated patterns (DAMPs) from necrotic host cells. Examples of PRRs are C-type lectin receptors and NOD-like receptors but probably the most well-known group of pattern recognition receptors are the Toll-like receptors (TLRs), which were discovered as homologs of the Toll receptor in Drosophila melanogaster (fruit fly) [20]. Also in fish, a large number of TLRs was identified, some of which are homologs of mammalian TLRs, although PAMP specificity has not always been confirmed [21]. Many PRRs drive intracellular signaling pathways that are MyD88 dependent and lead to the activation of nuclear factor kappa-light-chain-enhancer of activated $\mathrm{B}$ cells $(\mathrm{NF}-\mathrm{\kappa B})$ and the transcription of several proinflammatory genes in innate immune cells, including macrophages [reviewed by $(22,23])$. 
In this thesis we frequently use LPS as a typical PAMP to activate pro-inflammatory responses in carp macrophages. Mammalian macrophages are highly sensitive to LPS, which is mediated by the 'lipid A' recognized by a protein complex of TLR4 and myeloid differentiation factor 2 (MD-2) [24, 25]. Although tlr4 genes have been identified in zebrafish [26, 27] grass carp (Ctenopharyngodon idella) [28] and carp [29], the exact role of these Tlr4 genes in the recognition of LPS remains ambiguous. Studies in zebrafish, tetraodon (Tetraodon nigroviridis) and gilthead seabream (Sparus aurata) have suggested that LPS does not signal through Tlr4 in fish [30] and that Tlr4 even mediates downregulation of NF- $\kappa B$, which would explain the relative LPS insensitivity of fish when compared to mammals [31]. In contrast, early studies did report activation of MyD88 signaling pathways [32] and recent studies reported the presence of Md-2 in zebrafish. The expression of zebrafish Md-2 appears to correlate with expression of the macrophage marker mpeg1 and of Tlr4 [27]. Alternative LPS-recognition pathways have also been examined in various fish species including intracellular LPS recognition via Nod1 [33] and recognition by scavenger receptor B2a with subsequent Nod1 and Nod2 activation [34], but all with subsequent NF- $\mathrm{BB}$ activation. No matter what exact signaling pathway is activated, LPS stimulation still results in NF- $\kappa$ B activation and elicits inflammatory responses in carp innate immune cells [35-37] which led us to use this canonical PAMP to induce inflammatory responses in carp macrophages.

Other PAMPs but also DAMPs such as host DNA, as well as cytokines and chemokines released from cells in the environment can also activate and polarize macrophages, but the functions performed may be different from those stimulated with LPS. Signals from apoptotic cells and anti-inflammatory cytokines drive macrophages to show anti-inflammatory phenotypes [38-41]. For decades, scientist have grasped the importance of regulating inflammatory responses and in that process identified the second messenger cyclic adenosine monophosphate (cAMP) as important in mitigating these responses [42]. Over the years many studies have reported on the anti-inflammatory effects in various immune cells when intracellular cAMP is increased, primarily through interaction with NF- $\mathrm{BB}$ (extensively reviewed by [43]). Moreover, anti-inflammatory genes such as arginase are increased by the anti-inflammatory Il-4 through upregulation of intracellular cAMP [44]. Similarly, prostaglandins [44, 45] and adenosine [46] present at sites of inflammation or wound healing can elicit anti-inflammatory responses through increases of 
intracellular cAMP. This led us to use cAMP to induce anti-inflammatory responses in carp macrophages.

\section{Polarized macrophages: lessons from mammals}

The concept that macrophages can be driven to show seemingly opposite functions is known as macrophage polarization. Of particular interest is the capability of macrophages to polarize in the presence of only 'innate signals' such as LPS or increased intracellular cAMP as a result of signaling molecules such as interleukin (IL) 4 and prostaglandins. Other signals that drive macrophages to polarize or enhance already-polarized phenotypes include inflammatory cytokines such as interferon- $\gamma$, which is often associated with adaptive immune cells, as is IL-4. Their role is concisely described in the next paragraph for mammals and will be extensively reviewed in chapter 2 for fish.

In mammals, polarized macrophages were first described as M1 and M2 as functional innate extensions of polarized adaptive responses driven by Th1 and Th2 cells, respectively, and are also referred to as 'classically activated' (M1) or 'alternatively activated' (M2) [47] (Figure 1.2A). M1 macrophages (especially in mice) were, and still are, characterized by their production of $\mathrm{NO}$ and pro-inflammatory cytokines and M2 macrophages by their increased arginase activity and production of antiinflammatory cytokines. The differential use of L-arginine for the production of NO (by M1) or for production of prolines and polyamines via the enzyme arginase (by M2), placed these macrophages as functional opposites in the 'arginine fork'. Over time the relatively simple distinction between M1 and M2 has been refined, first by the introduction of the M2 macrophage subtypes M2a, M2b and M2c (Figure 1.2B) [39] and later by the introduction of differently-activated macrophages being part of a full spectrum (Figure 1.2C) [41, 48]. The nomenclature of different macrophage subsets within the full spectrum remains complex as every stimulus added to macrophages may result in a slightly different phenotype. Therefore, the proposition was made to adapt macrophage nomenclature to include the specific stimulus [for example M(LPS)]. One of the most advanced views on this matter is based on a three-dimensional visualization of macrophage transcriptomes after stimulation with many different stimuli showing that many of the frequentlyused pro- and anti-inflammatory stimuli each induce their own transcriptional profile, but clearly cluster together in groups of a similar nature [41]. For example, transcriptomes of macrophages stimulated with LPS, interferon- $\gamma$ (IFN- $\gamma$ ) or the 
combination of both, all cluster together towards one extreme of the spectrum, while transcriptomes of macrophages stimulated with IL-4, IL-13, and to a lesser degree also IL-10 and glucocorticoid hormones such as cortisol, all cluster towards the other extreme of the spectrum [41]. For clarity, in this thesis we have chosen to use M1 and M2 to refer to the extremes of the spectrum and indicate the specific stimuli used for polarization in our experiments. Throughout this thesis, we will compare polarized carp macrophages with known phenotypes of mammalian macrophages and scrutinize the M1 and M2 classification for carp macrophages.

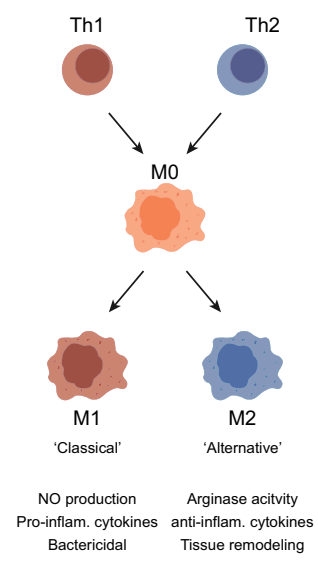

B

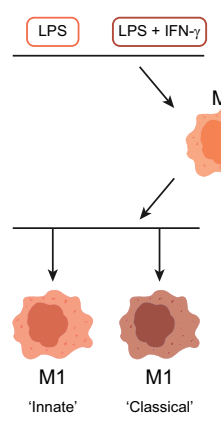

NO production
Pro-inflam. cytokines Bactericidal
C

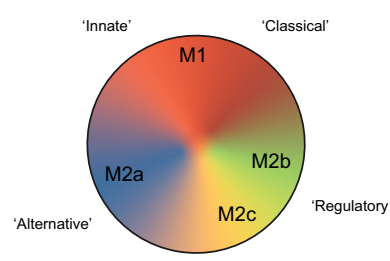

Figure 1.2 Macrophage polarization in mammals is more complex than just M1 and M2. Developing views on macrophage polarization. A) First, M1 and M2 macrophages were primarily viewed as the extension of polarized T helper (Th) cell responses. B) Then, M1 and M2 views expanded into multiple subsets which are often induced by the indicated stimuli $[39,40]$. Macrophage phenotypes are accompanied by their different nomenclature, functional significance and frequently used (in vitro) stimuli, including lipopolysaccharide (LPS), interferon- $\gamma$ (IFN- $\gamma$ ), interleukin-4 (IL-4), interleukin-13 (IL13), immune complexes (IC), toll-like receptor ligands (TLR-lig.), interleukin-10 (IL-10), transforming growth factor $\beta$ (TGF- $\beta$ ). C) Macrophage subtypes are now often viewed as part of a continuous spectrum of macrophage polarization states in which each stimulus induces a different phenotype, but similar phenotypes cluster together [41, 48]. Figure based on [39, 41, 47, 48].

Although macrophages from mice and humans can both be roughly classified as belonging to the same functional groups indicated in Figure 1.1B, clear differences in macrophage phenotypes also exist between these two well-studied species. These differences can at least in part be ascribed to the fact that murine macrophages are usually derived from precursors in bone marrow while human macrophages are most often differentiated from monocytes in the blood. One of 
the most striking differences between murine and human macrophage function is their use of L-arginine. While murine macrophages readily polarize to produce NO or show increased arginase activity, these hallmarks are debated in human macrophages [49]. Conversely, many of the pro-inflammatory cytokines produced after polarization towards M1 are shared between mouse and human. Welldocumented examples include IL-6, IL-12p35 and tumor necrosis factor- $\alpha$ [40]. Other responses shared between murine and human macrophages is the increase in expression of mannose receptor C-type 1 and transglutaminase-2 following activation by IL-4 [50]. Also comparable between mouse and human macrophages is the increase in the IL-4 receptor alpha chain (IL4RA) and suppressor of cytokine signaling 3 (SOCS3), both associated with IL-10-stimulated regulatory macrophages [40]. The degree of conservation between mammalian and carp macrophages will be addressed in this thesis. A relatively new and alternative approach to study macrophage polarization focusses on the differences in energy metabolism between M1 and M2 macrophages. In mammals, M1 macrophages have been shown to rely primarily on glycolysis for the generation of energy while M2 macrophage rely primarily on oxidative phosphorylation [51,52]. Whether these differences in energy metabolism also extend to fish, will be addressed in chapter 5 . Last but not least, a recent comparison of macrophage phenotypes from pig indicated that bone marrow derived pig macrophages were more similar to human monocyte-derived than to human bone marrow-derived macrophages, although only few markers were examined [53]. This could be of relevance to our study because we address macrophages derived from head kidney, the organ in fish considered equivalent to mammalian bone marrow.

\section{Preliminary evidence for the existence of polarized macrophages in fish}

Prior to the start of this thesis an increasing amount of functional evidence already suggested that polarized macrophages should also exist in fish, for which reason this thesis starts with a review of the state-of-the art (chapter 2). Indeed, several studies in various fish species already indicated the presence of inflammatory macrophages in response to microbial ligands such as LPS. In carp, early studies already indicated the production of NO and a number of pro-inflammatory cytokines by macrophages [35, 36]. Carp macrophages had been shown to increase arginase activity in response to cAMP [35] and a few years later, the discovery of an ancestral il-4/13 gene led to the study of Th2 related immune responses [54, 55]. This was followed by studies which compared Il-4/13-induced arginase activity 
with cAMP-induced arginase activity in goldfish (Carassius auratus) [56]. Moreover, increases in arginase activity were studied with a combination of Il-4/13 and LPS in grass carp [57] and later also with Il-4/13 alone in grass carp [58]. Taking another approach, in vivo tracking suggested the presence of M1 and M2-like phenotypes in zebrafish larvae based on the presence or absence of $\operatorname{tnf} \alpha(a)$ and expression studies on sorted mpeg+ macrophages showed the expression of some genes also reported for mammalian M1 and M2 macrophages [59, 60]. The great value of zebrafish for in vivo tracking studies however stands opposite to the difficulty of obtaining large numbers of zebrafish macrophages for gene transcription studies such as RNA sequencing. Here, the much larger size of carp provides the advantage of isolating large numbers of immature precursors from the head kidney, culturing them into mature macrophages and subsequently polarizing these macrophages to determine their respective phenotypes as described in this thesis.

\section{The challenges of the carp genome}

The value and level of detail one can obtain from studying gene expression profiles of polarized carp macrophages, heavily depends on the quality and annotation of the corresponding genome. Draft genomes of cyprinids including common carp have been assembled and annotated, always primarily based on the zebrafish genome. The common carp genome was first published in 2011 [61] with significant improvements published in 2012 and 2016 [62, 63] resulting in a draft genome with over 50.000 predicted genes. Only very recently an improved version of the common carp genome was released [64] and a further improvement based on long-read RNA sequencing is in the making (unpublished data), both very promising developments that came too late for the data in this thesis. Despite major improvements made over the years, polyploidy causes the presence of many duplicated genes which have complicated the assembly. At this moment, the predictions and annotations of the common carp genome have sometimes remained incomplete, particularly for genes that are rarely expressed.

Compared to other vertebrates, teleost fish are believed to have undergone an extra $\left(3^{\text {rd }}\right)$ round of whole genome duplication resulting in roughly a doubling of genes in most teleost fish, including zebrafish (3R). Carp (4R) amongst several other cyprinid species, has undergone a $4^{\text {th }}$ round of whole genome duplication relatively recently, around 12 million years ago [reviewed by (Petit et al., 2017)] often resulting in four copies for each gene found in mammals (see also Figure 1.1B). As recently reviewed, 
although sometimes duplicated genes are lost over evolution, $60-90 \%$ of the duplications are estimated to have been retained in the common carp genome [9]. Besides loss of function, gene duplications can also allow for sub functionalization (retention of different subsets of ancestral functions by different paralogs) or neofunctionalization (mutations allowing for a new function). As published only recently, the current hypothesis is that the carp genome can be divided into two sub-genomes, with one classified as the dominant genome [64]. Indeed, we sometimes noticed clear differences in basal- or induced expression of different paralogs as detected by quantitative PCR, which is likely due to dominance of one sub-genome over the other. However, upon first sight it looks like the number of genes $>2$ fold higher expressed in one of the two sub-genomes cannot consistently be ascribed to the same sub-genome. This indicates it may be hard to predict which paralog will be highest expressed and stresses the importance of studying all gene paralogs when studying common carp. Overall, different gene copy numbers, imperfect gene annotations and sometimes limited knowledge on functional aspects of signaling pathways well-described for mammals significantly challenges the direct comparison of particularly transcriptional studies from mammalian to fish macrophages.

\section{Implications for aquaculture}

Polarized immune responses, whether driven by different Th cell subsets or not, can be very effective or sometimes counter effective, depending on (the nature of) the pathogen and stage of infection. For example, different types of immune activity are often required to clear intracellular pathogens or extracellular pathogens. Furthermore, early phase immune activity during phases of exponential pathogen growth is often different from immune activity during lag and healing phases. The outcome of such host-pathogen interactions in vivo is complex and difficult to predict, let alone steer. However, one can imagine it could be of practical value to have the ability to balance sometimes unbalanced immune activities as for example, during exacerbated inflammation. Prior fundamental knowledge of innate immune responses in fish, including macrophage polarization, is important for practical application. So-called 'health feeds', specialized fish feeds which include constituents with assumed or proven immunomodulating effects, are increasingly becoming available to fish farmers. Immunomodulating activities of some of these added constituents can rely on them being sensed by pattern recognition receptors on, for example macrophages. Alternatively, they may rely on prebiotic effects 
on microbial populations and downstream sensing of short chain fatty acids of, for example macrophages. As a result, such macrophages may become 'trained' or 'polarized' with often beneficial and sometimes counter effective effects. It may be clear that a more detailed knowledge of these innate immune responses is not only informative but should be considered essential for implementation of immunomodulators in aquaculture. Therefore, the overall aim of this thesis is to characterize polarized innate immune responses in carp with the emphasis on macrophages.

\section{Outline of this thesis}

In the General introduction (chapter 1), we first discuss the relevance of innate immunity for common carp as a cultured fish species and the central role of macrophages in innate immune responses. We introduce macrophages as an innate immune cell type with high plasticity and discuss the concept of macrophage polarization as it has been characterized and defined for mammalian macrophages. Moreover, we provide a brief overview of the indications for the presence of polarized macrophages in carp and place the potential contribution of our studies in the context of aquaculture for the development of immunomodulators to improve fish health.

In chapter 2 we thoroughly review the existing literature on macrophage polarization in fish. Here, we review both the stimuli frequently used to polarize macrophages in mammals and discuss preliminary but promising markers to read out M1 and M2 macrophage responses in fish. We discuss the value of inos as a conserved marker for M1 and arginase 2 as a marker for M2 fish macrophages. Moreover, we provide first insights and criteria for additional M2 markers in fish.

In chapter 3 we set out to describe a comprehensive functional and transcriptional phenotype of polarized carp macrophages. We combine information on the established nitric oxide and arginase assays with morphological differences to first, confirm M1 and M2 macrophage polarization and second, use a sequencing approach to elucidate transcriptional profiles of these cells. The phenotypes of polarized carp macrophages proved strikingly similar in function to those described in mammals. We discuss how polarization towards M1 and M2 phenotypes using stimulators such as only LPS and cAMP strengthens the 'macrophages first' 
hypothesis. Finally, we describe an extensive set of potential marker genes able to discriminate between M1 and M2 carp macrophages.

In chapter 4 we study how M1 macrophages polarized with LPS contribute to neutrophil responses as the major producers of granulocyte colony stimulating factor (Csf3/G-csf). We study basal expression patters of two Csf3 paralogs in different organ and cell-types and pinpoint M1 macrophages as the major producers of Csf3. We characterize the function of the paralogue Csf3b as primarily growth factor for neutrophils and the function of Csf3a as an inducer of a more mixed phenotype of macrophages and neutrophils.

In chapter 5 we hypothesize that functional conservation of polarized macrophages in carp could also indicate conservation of associated energy metabolism. Therefore, we study the metabolic profiles of carp macrophages polarized towards M1 and M2 extremes. For this we do not only use enzymatic assays, but also realtime extracellular flux analysis (Seahorse) to determine oxidative phosphorylation and glycolysis. Here we conclude that carp M1 macrophages, similar to mammalian M1 macrophages, show impaired oxidative capacity while M2 macrophages do not.

In the General discussion (chapter 6) we discuss our findings primarily in light of the evolutionary conservation of macrophage polarization. We discuss the conservation and use of polarizing cytokines in carp macrophages as a next step to expand and refine our understanding of carp macrophage responses. Additionally, we apply our proposed M1 and M2 markers to elucidate a 'common inflammatory' phenotype and scrutinize their use as markers for in vivo studies. Finally, we discuss preliminary findings regarding the M2 side of the macrophage spectrum. 


\section{References}

1. $\quad$ FAO (2018) The State of Fisheries and Aquaculture in the world 2018

2. FAO (2019) FAO yearbook Fishery and Aquaculture Statistics 2017. FOOD AND AGRICULTURE ORGANIZATION OF THE UNITED NATIONS, Rome

3. Stentiford GD, Sritunyalucksana K, Flegel TW, Williams BAP, Withyachumnarnkul B, Itsathitphaisarn O, Bass D (2017) New Paradigms to Help Solve the Global Aquaculture Disease Crisis. PLOS Pathog 13:e1006160.

4. Ward AE, Rosenthal BM (2014) Evolutionary responses of innate immunity to adaptive immunity. Infect Genet Evol 21:492-496. doi:10.1016/j.meegid.2013.12.021

5. Flajnik MF, Kasahara M (2010) Origin and evolution of the adaptive immune system: genetic events and selective pressures. Nat Rev Genet 11:47-59. doi:10.1038/nrg2703

6. Magor BG, Magor KE (2001) Evolution of effectors and receptors of innate immunity. Dev Comp Immunol 25:651-682. doi:10.1016/S0145-305X(01)00029-5

7. Flajnik MF (2018) A cold-blooded view of adaptive immunity. Nat Rev Immunol 18:438-453. doi:10.1038/s41577-018-0003-9

8. Balon EK (1995) Origin and domestication of the wild carp, Cyprinus carpio: from Roman gourmets to the swimming flowers. Aquaculture 129:3-48. doi:10.1016/0044-8486(94)00227-F

9. Petit J, David L, Dirks R, Wiegertjes GF (2017) Genomic and transcriptomic approaches to study immunology in cyprinids: What is next? Dev Comp Immunol 75:48-62. doi:10.1016/j. dci.2017.02.022

10. Chen Z, Omori Y, Koren S, Shirokiya T, Kuroda T, Miyamoto A, Wada H, Fujiyama A, Toyoda A, Zhang S, Wolfsberg TG, Kawakami K, Phillippy AM, Mullikin JC, Burgess SM (2019) De novo assembly of the goldfish (\&lt;em\&gt;Carassius auratus \&lt;/em\&gt;) genome and the evolution of genes after whole-genome duplication. Sci Adv 5:eaav0547. doi:10.1126/sciadv.aav0547

11. Li J-T, Hou G-Y, Kong X-F, Li C-Y, Zeng J-M, Li H-D, Xiao G-B, Li X-M, Sun X-W (2015) The fate of recent duplicated genes following a fourth-round whole genome duplication in a tetraploid fish, common carp (Cyprinus carpio). Sci Rep 5:8199. doi:10.1038/srep08199

12. Ellis AE (2001) Innate host defense mechanisms of fish against viruses and bacteria. Dev Comp Immunol 25:827-839. doi:10.1016/S0145-305X(01)00038-6

13. Abram QH, Dixon B, Katzenback BA (2017) Impacts of Low Temperature on the Teleost Immune System. Biology (Basel) 6:39. doi:10.3390/biology6040039

14. Parham P (2003) Innate immunity: The unsung heroes. Nature 423:20. doi:10.1038/423020a

15. Medzhitov R, Janeway CJ (2000) Innate immune recognition: mechanisms and pathways. Immunol Rev 173:89-97. doi:10.1034/j.1600-065X.2000.917309.x

16. Bowdish DME, Loffredo MS, Mukhopadhyay S, Mantovani A, Gordon S (2007) Macrophage receptors implicated in the "adaptive" form of innate immunity. Microbes Infect 9:1680-1687. doi:10.1016/j.micinf.2007.09.002

17. Netea MG, Schlitzer A, Placek K, Joosten LAB, Schultze JL (2019) Innate and Adaptive Immune Memory: an Evolutionary Continuum in the Host's Response to Pathogens. Cell Host Microbe 25:13-26. doi:10.1016/j.chom.2018.12.006 
18. Petit J, Embregts CWE, Forlenza M, Wiegertjes GF (2019) Evidence of Trained Immunity in a Fish: Conserved Features in Carp Macrophages. J Immunol 203(1):216-224. doi:10.4049/ jimmunol.1900137

19. Aderem A, Ulevitch RJ (2000) Toll-like receptors in the induction of the innate immune response. Nature 406:782-787. doi:10.1038/35021228

20. Anderson K, Jürgens G, Nüsslein-Volhard C (1985) Establishment of dorsal-ventral polarity in the Drosophila embryo: Genetic studies on the role of the Toll gene product. Cell 42:779-789. doi:10.1016/0092-8674(85)90274-0

21. Rebl A, Goldammer T, Seyfert H-M (2010) Toll-like receptor signaling in bony fish. Vet Immunol Immunopathol 134:139-150. doi:10.1016/j.vetimm.2009.09.021

22. Akira S, Takeda K (2004) Toll-like receptor signalling. Nat Rev Immunol 4:499-511. doi:10.1038/ nri1391

23. Kawasaki T, Kawai T (2014) Toll-like receptor signaling pathways. Front Immunol 5:461. doi:10.3389/fimmu.2014.00461

24. Poltorak A, He X, Smirnova I, Liu M-Y, Huffel C Van, Du X, Birdwell D, Alejos E, Silva M, Galanos C, Freudenberg M, Ricciardi-Castagnoli P, Layton B, Beutler B (1998) Defective LPS Signaling in C3H/HeJ and C57BL/10ScCr Mice: Mutations in Tlr4 Gene. Science (80- ) 282:2085 LP - 2088. doi:10.1126/science.282.5396.2085

25. Raetz CRH, Whitfield C (2002) Lipopolysaccharide Endotoxins. Annu Rev Biochem 71:635-700. doi:10.1146/annurev.biochem.71.110601.135414

26. Sullivan C, Charette J, Catchen J, Lage CR, Giasson G, Postlethwait JH, Millard PJ, Kim CH (2009) The Gene History of Zebrafish tlr4a and tlr4b Is Predictive of Their Divergent Functions. $J$ Immunol 183:5896 LP - 5908. doi:10.4049/jimmunol.0803285

27. Loes AN, Hinman MN, Farnsworth DR, Miller AC, Guillemin K, Harms MJ (2019) Identification and characterization of zebrafish Tlr4 co-receptor Md-2. bioRxiv 817528. doi:10.1101/817528

28. Huang R, Dong F, Jang S, Liao L, Zhu Z, Wang Y (2012) Isolation and analysis of a novel grass carp toll-like receptor 4 (tlr4) gene cluster involved in the response to grass carp reovirus. Dev Comp Immunol 38:383-388. doi:10.1016/j.dci.2012.06.002

29. Pietretti D, Forlenza M, Fink IR, Wiegertjes G (2013) A closer look at Toll-like receptor 4 (TLR4) and toll-like receptor 20 (TLR20) of common carp (Cyprinus carpio). Fish Shellish Immunol 34:1673. doi:10.1016/j.fsi.2013.03.121

30. Sepulcre MP, Alcaraz-Pérez F, López-Muñoz A, Roca FJ, Meseguer J, Cayuela ML, Mulero V (2009) Evolution of Lipopolysaccharide (LPS) Recognition and Signaling: Fish TLR4 Does Not Recognize LPS and Negatively Regulates NF-кB Activation. J Immunol 182:1836 LP - 1845. doi:10.4049/jimmunol.0801755

31. Swain P, Nayak SK, Nanda PK, Dash S (2008) Biological effects of bacterial lipopolysaccharide (endotoxin) in fish: A review. Fish Shellfish Immunol 25:191-201. doi:10.1016/j.fsi.2008.04.009

32. van der Sar AM, Stockhammer OW, van der Laan C, Spaink HP, Bitter W, Meijer AH (2006) MyD88 Innate Immune Function in a Zebrafish Embryo Infection Model. Infect Immun 74:2436 LP - 2441. doi:10.1128/IAI.74.4.2436-2441.2006 
33. Bi D, Wang Y, Gao Y, Li X, Chu Q, Cui J, Xu T (2018) Recognition of Lipopolysaccharide and Activation of NF- $\kappa B$ by Cytosolic Sensor NOD1 in Teleost Fish. Front. Immunol. 9:1413

34. Lu X-J, Ning Y-J, Liu H, Nie L, Chen J (2018) A Novel Lipopolysaccharide Recognition Mechanism Mediated by Internalization in Teleost Macrophages. Front. Immunol. 9:2758

35. Joerink M, Ribeiro CMS, Stet RJM, Hermsen T, Savelkoul HFJ, Wiegertjes GF (2006) Head Kidney-Derived Macrophages of Common Carp (Cyprinus carpio L.) Show Plasticity and Functional Polarization upon Differential Stimulation. J Immunol 177:61-69. doi:10.4049/ jimmunol.177.1.61

36. Arts JAJ, Tijhaar EJ, Chadzinska M, Savelkoul HFJ, Verburg-van Kemenade BML (2010) Functional analysis of carp interferon-: Evolutionary conservation of classical phagocyte activation. Fish Shellfish Immunol 29:793-802. doi:10.1016/J.FSI.2010.07.010

37. Piazzon MC, Savelkoul HFJ, Pietretti D, Wiegertjes GF, Forlenza M (2015) Carp Il10 Has Anti-Inflammatory Activities on Phagocytes, Promotes Proliferation of Memory T Cells, and Regulates B Cell Differentiation and Antibody Secretion. J Immunol 194:187-199. doi:10.4049/ jimmunol.1402093

38. Martinez FO, Helming L, Milde R, Varin A, Melgert BN, Draijer C, Thomas B, Fabbri M, Crawshaw A, Ho LP, Ten Hacken NH, Cobos Jiménez V, Kootstra NA, Hamann J, Greaves DR, Locati M, Mantovani A, Gordon S (2013) Genetic programs expressed in resting and IL-4 alternatively activated mouse and human macrophages: similarities and differences. Blood 121:e57-e69. doi:10.1182/blood-2012-06-436212

39. Mantovani A, Sica A, Sozzani S, Allavena P, Vecchi A, Locati M (2004) The chemokine system in diverse forms of macrophage activation and polarization. Trends Immunol 25:677-686. doi:10.1016/j.it.2004.09.015

40. Murray PJ, Allen JE, Biswas SK, Fisher EA, Gilroy DW, Goerdt S, Gordon S, Hamilton JA, Ivashkiv LB, Lawrence T, Locati M, Mantovani A, Martinez FO, Mege J-L, Mosser DM, Natoli G, Saeij JP, Schultze JL, Shirey KA, Sica A, Suttles J, Udalova I, van Ginderachter JA, Vogel SN, Wynn TA (2014) Macrophage activation and polarization: nomenclature and experimental guidelines. Immunity 41:14-20. doi:10.1016/j.immuni.2014.06.008

41. Xue J, Schmidt S V, Sander J, Draffehn A, Krebs W, Quester I, De Nardo D, Gohel TD, Emde M, Schmidleithner L, Ganesan H, Nino-Castro A, Mallmann MR, Labzin L, Theis H, Kraut M, Beyer M, Latz E, Freeman TC, Ulas T, Schultze JL (2014) Transcriptome-based network analysis reveals a spectrum model of human macrophage activation. Immunity 40:274-288. doi:10.1016/j. immuni.2014.01.006

42. Bourne HR, Weinstein Y, Melmon KL, Lichtenstein LM, Henney CS, Shearer GM (1974) Modulation of Inflammation and Immunity by Cyclic AMP. Science (80- ) 184:19 LP - 28. doi:10.1126/science.184.4132.19

43. Gerlo S, Kooijman R, Beck IM, Kolmus K, Spooren A, Haegeman G (2011) Cyclic AMP: a selective modulator of NF- $\kappa B$ action. Cell Mol Life Sci 68:3823-3841. doi:10.1007/s00018-011-0757-8

44. Corraliza IM, Soler G, Eichmann K, Modolell M (1995) Arginase Induction by Suppressors of Nitric Oxide Synthesis (IL-4, IL-10 and PGE2) in Murine Bone-Marrow-Derived Macrophages. Biochem Biophys Res Commun 206:667-673. doi:10.1006/bbrc.1995.1094 
45. Luan B, Yoon Y-S, Le Lay J, Kaestner KH, Hedrick S, Montminy M (2015) CREB pathway links PGE2 signaling with macrophage polarization. Proc Natl Acad Sci 112:15642 LP - 15647. doi:10.1073/pnas.1519644112

46. Csóka B, Selmeczy Z, Koscsó B, Németh ZH, Pacher P, Murray PJ, Kepka-Lenhart D, Morris Jr SM, Gause WC, Leibovich SJ, Haskó G (2012) Adenosine promotes alternative macrophage activation via A2A and A2B receptors. FASEB J 26:376-386. doi:10.1096/fj.11-190934

47. Mills CD, Kincaid K, Alt JM, Heilman MJ, Hill AM (2000) M-1/M-2 Macrophages and the Th1/ Th2 Paradigm. J Immunol. https://doi.org/10.4049/jimmunol.164.12.6166

48. Mills CD, Ley K (2014) M1 and M2 Macrophages: The Chicken and the Egg of Immunity. 92037:716-726. doi:10.1159/000364945

49. Thomas AC, Mattila JT (2014) "Of mice and men": arginine metabolism in macrophages. Front Immunol 5:479. doi:10.3389/fimmu.2014.00479

50. Spiller KL, Wrona EA, Romero-Torres S, Pallotta I, Graney PL, Witherel CE, Panicker LM, Feldman RA, Urbanska AM, Santambrogio L, Vunjak-Novakovic G, Freytes DO (2016) Differential gene expression in human, murine, and cell line-derived macrophages upon polarization. Exp Cell Res 347:1-13. doi:10.1016/j.yexcr.2015.10.017

51. Van den Bossche J, Baardman J, de Winther MPJ (2015) Metabolic Characterization of Polarized M1 and M2 Bone Marrow-derived Macrophages Using Real-time Extracellular Flux Analysis. J Vis Exp 53424. doi:10.3791/53424

52. Van den Bossche J, Baardman J, Otto NA, van der Velden S, Neele AE, van den Berg SM, LuqueMartin R, Chen H-J, Boshuizen MCS, Ahmed M, Hoeksema MA, de Vos AF, de Winther MPJ (2016) Mitochondrial Dysfunction Prevents Repolarization of Inflammatory Macrophages. Cell Rep 17:684-696. doi:10.1016/j.celrep.2016.09.008

53. Gao J, Scheenstra MR, van Dijk A, Veldhuizen EJA, Haagsman HP (2018) A new and efficient culture method for porcine bone marrow-derived M1- and M2-polarized macrophages. Vet Immunol Immunopathol 200:7-15. doi:10.1016/j.vetimm.2018.04.002

54. Dijkstra JM, Grimholt U, Leong J, Koop BF, Hashimoto K (2013) Comprehensive analysis of MHC class II genes in teleost fish genomes reveals dispensability of the peptide-loading DM system in a large part of vertebrates. BMC Evol Biol 13:260. doi:10.1186/1471-2148-13-260

55. Yamaguchi T, Takizawa F, Fischer U, Dijkstra J (2015) Along the Axis between Type 1 and Type 2 Immunity; Principles Conserved in Evolution from Fish to Mammals. Biology (Basel) 4:814-859. doi:10.3390/biology4040814

56. Hodgkinson JW, Fibke C, Belosevic M (2017) Recombinant IL-4/13A and IL-4/13B induce arginase activity and down-regulate nitric oxide response of primary goldfish (Carassius auratus L.) macrophages. Dev Comp Immunol 67:377-384. doi:10.1016/j.dci.2016.08.014

57. Yang Z-J, Li C-H, Chen J, Zhang H, Li M-Y, Chen J (2016) Molecular characterization of an interleukin-4/13B homolog in grass carp (Ctenopharyngodon idella) and its role in fish against Aeromonas hydrophila infection. Fish Shellfish Immunol 57:136-147. doi:10.1016/j. fsi.2016.08.022 
58. Yang K, Feng S, Zhang S, Yin L, Zhou H, Zhang A, Wang X (2019) Characterization of a new il-4/13 homologue in grass carp (Ctenopharyngodon idella) and its cooperation with M-CSF to promote macrophage proliferation. Fish Shellfish Immunol 93:508-516. doi:10.1016/j. fsi.2019.07.070

59. Nguyen-Chi M, Laplace-Builhe B, Travnickova J, Luz-Crawford P, Tejedor G, Phan QT, DurouxRichard I, Levraud JP, Kissa K, Lutfalla G, Jorgensen C, Djouad F (2015) Identification of polarized macrophage subsets in zebrafish. Elife 4:1-14. doi:10.7554/eLife.07288

60. Rougeot J, Torraca V, Zakrzewska A, Kanwal Z, Jansen H, Spaink HP, Meijer AH (2019) RNAseq profiling of leukocyte populations in zebrafish larvae reveals a cxcl11 chemokine gene as a marker of macrophage polarization during mycobacterial infection. bioRxiv 554808 . doi:10.1101/554808

61. Zhang Y, Stupka E, Henkel C V, Jansen HJ, Spaink HP, Verbeek FJ (2011) Identification of Common Carp Innate Immune Genes with Whole-Genome Sequencing and RNA-Seq Data. J. Integr. Bioinform. 8:165. doi: 10.2390/biecoll-jib-2011-169

62. Henkel C V, Dirks RP, Jansen HJ, Forlenza M, Wiegertjes GF, Howe K, van den Thillart GEEJM, Spaink HP (2012) Comparison of the exomes of common carp (Cyprinus carpio) and zebrafish (Danio rerio). Zebrafish 9:59-67. doi:10.1089/zeb.2012.0773

63. Kolder ICRM, van der Plas-Duivesteijn SJ, Tan G, Wiegertjes GF, Forlenza M, Guler AT, Travin DY, Nakao M, Moritomo T, Irnazarow I, den Dunnen JT, Anvar SY, Jansen HJ, Dirks RP, Palmblad M, Lenhard B, Henkel C V, Spaink HP (2016) A full-body transcriptome and proteome resource for the European common carp. BMC Genomics 17:701. doi:10.1186/s12864-016-3038-y

64. Xu P, Xu J, Liu G, Chen L, Zhou Z, Peng W, Jiang Y, Zhao Z, Jia Z, Sun Y, Wu Y, Chen B, Pu F, Feng J, Luo J, Chai J, Zhang H, Wang H, Dong C, Jiang W, Sun X (2019) The allotetraploid origin and asymmetrical genome evolution of the common carp Cyprinus carpio. Nat Commun 10:4625. doi:10.1038/s41467-019-12644-1 



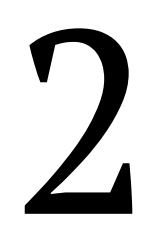

\section{Polarization of immune responses in fish: the 'macrophages first' point of view}

Geert F. Wiegertjes ${ }^{1}$, Annelieke S. Wentzel ${ }^{1}$, Herman P. Spaink ${ }^{2}$, Philip M. Elks $^{3}$ and Inge R. Fink ${ }^{1}$

${ }^{1}$ Cell Biology and Immunology Group, Wageningen Institute of Animal Sciences, Wageningen University, Wageningen, The Netherlands

${ }^{2}$ Institute of Biology, Leiden University, Leiden, The Netherlands

${ }^{3}$ Department of Infection and Immunity, University of Sheffield, Sheffield, South Yorkshire, United Kingdom 


\begin{abstract}
In this review, we support taking polarized immune responses in teleost fish from a 'macrophage first' point of view, a hypothesis that reverts the dichotomous $\mathrm{T}$ helper $\left(\mathrm{T}_{\mathrm{H}} 1\right.$ and $\mathrm{T}_{\mathrm{H}} 2$ driving forces by building on the idea of conservation of innate immune responses in lower vertebrates. It is plausible that the initial trigger for macrophage polarization into M1 (inflammation) or M2 (healing) could rely only on sensing microbial/parasite infection or other innate danger signals, without the influence of adaptive immunity. Given the long and ongoing debate on the presence/absence of a typical $T_{H} 1$ cytokine environment and, in particular, $T_{H} 2$ cytokine environment in fish immune responses, it stands out that the presence of macrophages with polarized phenotypes, alike M1 and M2, have been relatively easy to demonstrate for fish. We summarize in short present knowledge in teleost fish on those cytokines considered most critical to the dichotomous development of $\mathrm{T}_{\mathrm{H}} 1 / \mathrm{M} 1$ and $\mathrm{T}_{\mathrm{H}} 2 / \mathrm{M} 2$ polarization, in particular, but not exclusively, interferon- $\gamma$ and interleukin (IL)-4/IL-13. We review, in more detail, polarization of fish immune responses taken from the macrophage point of view for which we adopted the simple nomenclature of M1 and M2. We discuss inducible nitric oxide synthase, or NOS-2, as a reliable M1 marker and arginase-2 as a reliable M2 marker for teleost fish and discuss the value of these macrophage markers for the generation of zebrafish reporter lines to study M1/M2 polarization in vivo.
\end{abstract}




\section{Introduction}

Traditionally, polarization of immune responses into inflammatory and antiinflammatory types of immunity have been based on the presence of polarizing cytokine profiles secreted by, among others, T helper $1\left(\mathrm{~T}_{\mathrm{H}} 1\right)$ and $\mathrm{T}$ helper $2\left(\mathrm{~T}_{\mathrm{H}} 2\right)$ cell types. It is well-accepted that the presence in mice and humans of these polarizing $\mathrm{T}$ cell-derived cytokines, including interferon gamma (IFN- $\left.\gamma ; \mathrm{T}_{\mathrm{H}} 1\right)$ and interleukin-4 and -13 (IL-4/-13; $\mathrm{T}_{\mathrm{H}} 2$ ) can drive in vitro cultures of bone marrowderived macrophages from mice, and differentiated peripheral blood monocytes from humans, into polarized populations referred to as classically activated and alternatively activated, respectively [1]. In this concept, classically-activated macrophages are induced and enhanced by an inflammatory $\mathrm{T}_{\mathrm{H}} 1$ cytokine environment, whereas alternatively-activated macrophages are induced and enhanced by an anti-inflammatory $\mathrm{T}_{\mathrm{H}} 2$ cytokine environment. To mirror the $\mathrm{T}$ helper dichotomy [2], classically-activated macrophages are also simply referred to as M1 and alternatively-activated macrophages referred to as M2 macrophages.

Over the years the traditional view on the exact role of macrophages in polarized immune responses has been frequently fine-tuned to incorporate innate-activated macrophages next to the IFN- $\gamma$ driven classically-activated M1 and to incorporate next to IL-4/-13 stimulated M2 macrophages (also called M2a), macrophages coactivated by immune complexes or apoptotic cells (called M2b) or macrophages de-activated by glucocorticoids, transforming growth factor (TGF)- $\beta$, or IL-10 (called M2c), the latter also referred to as regulatory macrophages [3]. Recently, the somewhat over-simplified M1/M2 classification has been challenged by transcriptome comparisons of human monocyte-derived macrophages, identifying a large array of macrophage activation states and profiles [4]. Indeed, M1 and M2 macrophages may simply represent the extremes of the large scale of macrophage activation states [5]. As a reasonable compromise, the use of a common framework for macrophage-activation nomenclature based on i) a defined set of standards encompassing the source of macrophages (e.g. bone marrow-derived versus monocyte-derived), ii) definition of the activators (e.g. IFN- $\gamma$ versus IL-4/-13), and iii) a consensus collection of markers to describe macrophage activation, would unify experimental standards for diverse experimental scenarios in a more detailed manner [6]. The latter, however, applies primarily to human and mouse macrophages and thus for this review we have chosen to adopt the definition of M1 and M2 as groups of stimulated macrophages, combining several stimuli 


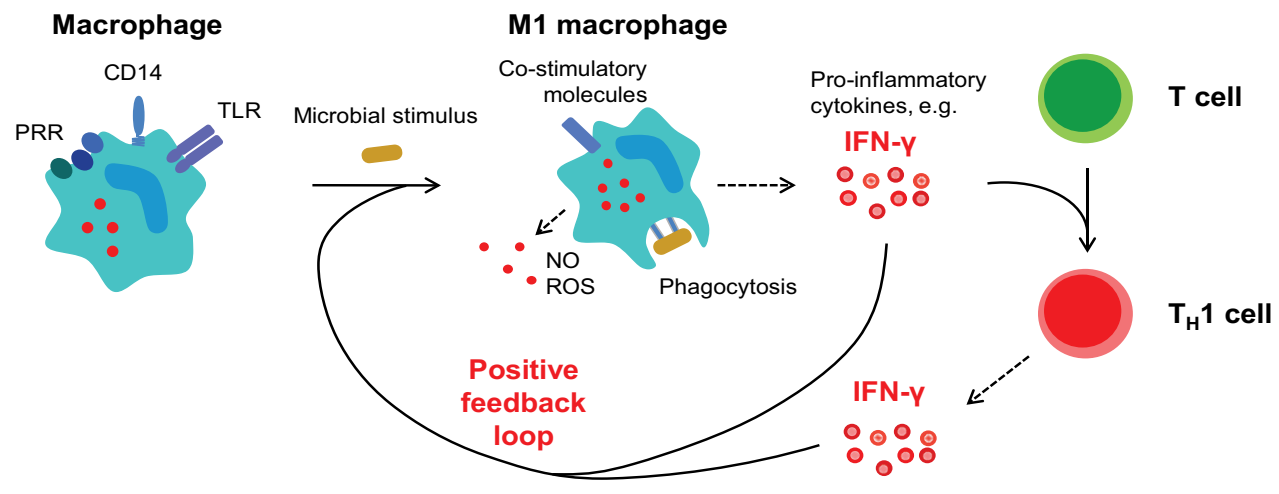

\section{Macrophage}

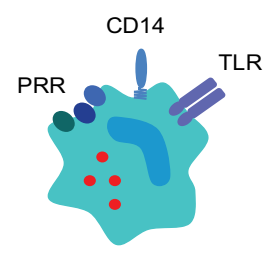

M2 macrophage

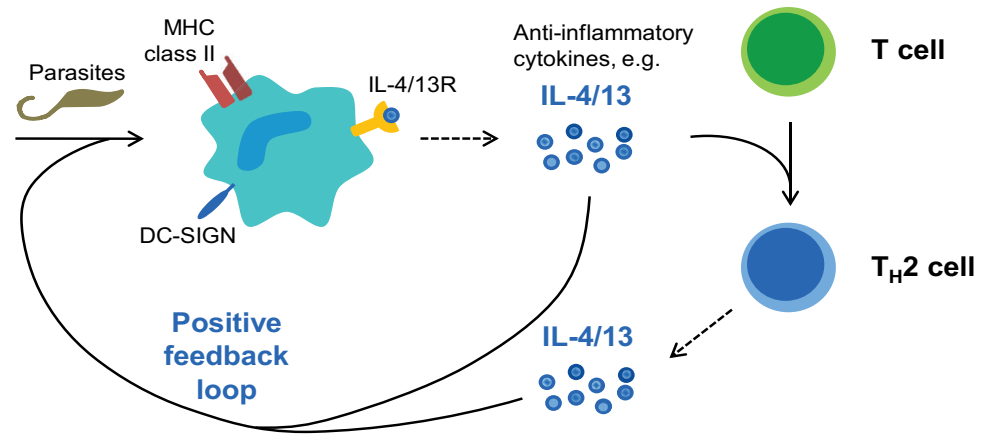

Figure 2.1 'Macrophage first' point of view: polarized immune responses are initiated by activation of macrophages and sustained by TH1 (IFN- $\gamma$ ) and TH2 (IL-4/13) cytokines.

Microbial stimuli are sensed by macrophages through Toll-like receptors (TLRs) along with CD14, or other pattern recognition receptors (PRR). Sensing of microbial stimuli (e.g. LPS from Gram-negative bacteria) leads to the development of innate-activated M1 macrophages with increased phagocytic activity, production of reactive oxygen species (ROS), nitric oxide (NO) and pro-inflammatory cytokines, among which IFN- $\gamma$. This cytokine drives T cells into a TH1 response, and in a feedback loop IFN- $\gamma$ induces classically-activated M1 macrophages characterized by a higher expression of MHC class II and co-stimulatory molecules in addition to an increase of the inflammatory functions already described for innate-activated M1 macrophages.

Parasite-related stimuli (e.g. chitin from helminths) are sensed by macrophages, leading to the development of alternatively-activated M2 macrophages. These have increased arginase activity, associated decreased microbicidal activity, and increased production of collagen and polyamines for cell growth and healing processes. T cells are skewed toward a TH2 profile and in a feedback loop, the TH2 cytokines IL-4 and/or IL-13 further induce alternatively-activated M2 macrophages which express DC-SIGN as well as higher levels of MHC class II molecules, in addition to an increase of the antiinflammatory functions already described. 
able to induce a similar functional profile [7]. No matter what, independent of the exact name and number of macrophage sub-classifications, the consensus is that polarized populations of macrophages are present during $\mathrm{T}_{\mathrm{H}}$-driven immune responses.

One current view is challenging the traditional paradigm of analyzing polarized immune responses by reverting the initial driving forces behind polarized immune responses, IFN- $\gamma$ and IL-4/-13, and by building on the importance of innate immunity, taking polarized immune responses from a 'macrophage first' point of view $[5,6]$. In this view, M1 or M2 macrophages would differentiate first during an immune response and subsequently direct $T$ lymphocytes to produce $T_{H} 1$ or $\mathrm{T}_{\mathrm{H}} 2$ responses, respectively, to amplify, rather than initiate, M1/M2 macrophage polarization in positive feed-back loops (Fig. 2.1). This would imply that the initial trigger for macrophage polarization could simply rely on sensing microbial/parasite infection or innate danger signals alone, without the necessary influence of adaptive immunity. Of course, the 'macrophage first' view does not exclude that $\mathrm{T}_{\mathrm{H}} 1$ and $\mathrm{T}_{\mathrm{H}} 2$ cytokine profiles amplify macrophage M1/M2 dichotomy, finally resulting in a selfsustaining $\mathrm{T}_{\mathrm{H}} 1-\mathrm{M} 1 / \mathrm{T}_{\mathrm{H}} 2-\mathrm{M} 2$ polarization during complex immune responses.

It is likely that macrophages are able to perform their main properties including phagocytosis, endocytosis, secretion and microbial killing in the steady state, with M1 and M2 polarizations contributing to a further modulation and tuning of immune responses [6]. Also a very attractive point of view is that M2 'heal' states could be the default mode of tissue macrophages with M1 states developing only under critical conditions into a potentially terminal phenotype [5]. In this respect it will be important to examine in future studies exactly which differences exist between steady state and M2 macrophages. It will also be important to determine if all activated macrophages can be grouped within M1 and M2 designations, especially with regard to macrophage phenotypes induced by non-T cell drivers such as those derived from innate immunity. Following the fate of individual macrophages in vivo, for example in live zebrafish (Danio rerio) (see also 'future developments'), may help examine if one-directional transition states from M2 to M1 exist. 
It is possible that the M1-M2 dichotomy arose early in evolution, prior to the development of mammalian vertebrates and could be an evolutionary conserved, intrinsic property of macrophages associated with transitions from healing (M2) to inflammation (M1) [5]. Comparative immunologists would tend to favor the 'macrophage first' view simply based on the fact that the ability of macrophage-like cell types to phagocytize foreign objects and repair cellular damage already existed in the first primitive animals, whereas the requirement for cytokine-mediated adaptive immunity developed only later in evolution [8]. Teleost fish are among the evolutionarily oldest vertebrates with both an innate and classical adaptive immune system, and are crucially important for studies on evolutionarily conserved functions of the immune system [9]. Examining macrophage function in teleost fish is highly interesting in particular with respect to determining the effects of T-cell derived cytokines on macrophage polarization. There has been a long-standing debate, which is still ongoing [10], about whether immune responses in fish can be characterized as truly polarized based on the presence/absence of a typical $\mathrm{T}_{\mathrm{H}} 1$ cytokine environment and, in particular, a typical $\mathrm{T}_{\mathrm{H}} 2$ cytokine environment [1113]. In contrast to the ongoing discussion on the presence of polarized $T_{H}$ subsets in fish, the presence of macrophages with polarized profiles alike mammalian M1 and M2 [14-16] has been relatively easy to demonstrate.

We summarize, in short, the present knowledge in teleost fish on those cytokines considered most critical to the development of M1 and M2, in particular but not exclusively, IFN- $\gamma$ and IL-4/-13. We discuss, in more detail, polarization of immune responses in fish taken from the macrophage point of view for which, for the purpose of this review, we have chosen to adopt the simple and informative [6], although sometimes confusing [6], nomenclature of M1 and M2. We will discuss the use of inducible nitric oxide synthase (iNOS) and arginase which, although sometimes debated [17], have proven useful markers to discriminate between mammalian M1 and M2 [18], but also appear useful markers of M1 and M2 of common carp [16]. The enzymes iNOS and arginase, by competing for L-arginine as substrate for both M1 and M2, in a process sometimes referred to as the arginine-fork, determine the balance between 'inflammatory' M1 type of macrophages and 'healing' M2 type of macrophages. We discuss evidence for the use of iNOS(B), or NOS-2(B), as marker for M1 and the use of arginase-2 as marker for M2 macrophages of teleost fish. 


\section{M1 macrophages in fish}

Notably, macrophages can be activated by microbial infection or innate danger signals without any influence of adaptive immune cells, leading to a form of M1 macrophages also defined as innate-activated [19]. These microbial stimuli can activate macrophages in an innate manner via detection through a large array of pattern recognition receptors (PRRs) [20]. Here, it is not the intention to extensively review the detection of these innate signals by PRRs in fish, for which several reviews exist already discussing the presence and function of, among others, Toll-like and viral nucleic acid receptors [21-29]. Overall, M1 macrophages can be grouped [6] as to include macrophages activated by bacterial PAMPs, of which probably the most frequently studied is Gram-negative bacterial lipopolysaccharides (LPS), macrophages activated by (microbial ligands in combination with) IFN- $\gamma$ and macrophages activated by granulocyte macrophage colony stimulating factor (GMCSF).

LPS from Gram-negative bacteria such as Escherichia coli probably are among the best-studied microbial stimuli. Despite the fact that a TLR4 receptor complex, as we know it from mammals, may not be functional in fish [30, 31], it is not especially relevant to understanding M1 activation whether LPS is sensed by a true TLR4 receptor complex or by other receptors sensing possible contaminations with peptidoglycan and/or DNA in impure LPS preparations [32] as the final activation state will still fit the M1 profile. In mammals, sensing of LPS by the TLR4 complex [33] will lead to activation of the relevant transcription factors, including nuclear factor kappa-light-chain-enhancer of activated B cells (NFKB) and activator protein 1 (AP-1), and downstream up-regulation of expression of pro-inflammatory cytokines, including IFN- $\gamma$ and tumor necrosis factor (TNF)- $\alpha$, resulting in the development of M1-like phenotypes. There are numerous publications that show that in vitro stimulation of fish macrophages with LPS leads to increased respiratory burst activity and associated production of oxygen radicals, production of nitrogen radicals and secretion of pro-inflammatory cytokines [34]. Thus, regardless of exactly which receptor senses exactly what compound in LPS preparations and subsequently leads to activation of macrophages, in vitro studies with LPS have clearly proven the existence of innate activated (M1) macrophages in fish. 
IFN- $\gamma$, as a combination stimulus together with LPS, forms the best characterized inflammatory (M1) side of the balance in the M1/M2 paradigm. LPS+IFN- $\gamma$ (classically) activated macrophages have higher respiratory burst activity and nitric oxide synthase expression as well as increased ability for antigen presentation (major histocompatibility complex (MHC) class II) and co-stimulation (CD86) than have LPS-only stimulated macrophages [20]. Gene expression profiles induced by either LPS or IFN- $\gamma$ on one hand, or a combination of LPS+IFN- $\gamma$ on the other, show a large degree of overlap but also enough differences not to consider each of these stimuli homologous, at least not for human macrophages [35, 36]. The promoter of mouse inducible nitric oxide (iNOS) contains two regions termed RI and RII of which both are mediated by LPS but only RII is mediated by IFN- $\gamma$, thus amplifying the effect of LPS $[37,38]$. The 'killing' profiles of innate- and classically-activated macrophages groups them together as M1 macrophages.

Members of the type II IFN family have been well characterized for fish [13, 39]. Recombinant grass carp (Ctenopharyngodon idella) IFN- $\gamma$ alone boosts NO production of monocytes/macrophages [40] whereas recombinant common carp (Cyprinus carpio) IFN- $\gamma$, as a combination stimulus with LPS, stimulates a powerful synergistic gene expression of pro-inflammatory cytokines and iNOS, and induces an enhanced respiratory burst activity and nitric oxide production [41]. Similar to the mammalian immune system, fish $\mathrm{T}$ cells and natural killer cells could be likely producers of IFN $-\gamma$. Furthermore, recombinant IFN $-\gamma$ appears to signal via a STAT1-dependent mechanism to increase expression of MHC class I and class II molecules, increase expression of several chemokines and cytokines, among which IL-1 $\beta$, IL-6, IL-12 and TNF- $\alpha$, and increase phagocytosis, oxidative burst and nitric oxide response of several fish species [reviewed by 13], all characteristics of proinflammatory responses linked with M1 macrophages. Similar to the mammalian situation, suppressor of cytokine signaling (SOCS)-1 is important for the control of IFN- $\gamma$ signaling [42]. It would be interesting, in this context, to study gene expression profiles of LPS- versus LPS + IFN- $\gamma$-stimulated fish macrophages in more detail to determine the degree of overlap in activation profile and conservation with mammalian M1 macrophages.

In contrast to mammals, the fish IFN- $\gamma$ family consists of two members; IFN- $\gamma$ and IFN- $\gamma$-related (IFN- $\gamma$ rel), although this is probably not of direct relevance to M1/M2 polarization. The distinction made between 'true' IFN- $\gamma$ and IFN- $\gamma$ rel is primarily 
based on a lack of nuclear localization signal in IFN- $\gamma$ rel sequences. Deletion in rainbow trout (Oncorhynchus mykiss) IFN- $\gamma$ of this particular site abolished its ability to induce chemokine expression (Zou et al 2005), in line with the essential role played by mammalian IFN- $\gamma$. Recombinant goldfish (Carassius auratus) IFN$\gamma$ rel elicits a robust but relatively short-lived priming of monocytes for respiratory burst, and down-regulates the priming potential of IFN- $\gamma$ and of TNF- $\alpha$ [43, 44]. Although knock-down studies in zebrafish indicated partly overlapping functions based on the fact that knock-down of either IFN- $\gamma$ isoform did not interfere with the ability to clear bacterial infection in vivo [45], recombinant common carp IFN- $\gamma$ rel fails to prime antimicrobial activity in phagocytes [41]. The most detailed studies on IFN- $\gamma$ rel have been performed on another cyprinid fish species; Ginbuna crucian carp (Carassius auratus langsdorfii), where two IFN- $\gamma$ rel genes exist. Recombinant IFN $\gamma$-rel1 and IFN- $\gamma$ rel2 both showed high antiviral activity. The fact that both IFN- $\gamma$ rel molecules exhibit biological activity as monomers [46] and not in the homodimeric conformation usual for IFN- $\gamma$, confirms that IFN- $\gamma$ rel molecules are likely to have biological activity different from IFN- $\gamma$. Most probably IFN- $\gamma$ rel proteins are antiviral proteins without direct effects on M1/M2 polarization in fish.

Once activated, M1 macrophages are characterized by cell-mediated immunity and the capacity to kill ingested and intracellular pathogens; a status driven and/ or reinforced, by a series of pro-inflammatory cytokines [3, 47, 48]. It is therefore difficult to assign to one of these cytokines in particular a more prominent role as a marker for M1 macrophages, but here we have chosen to shortly discuss the role of TNF- $\alpha$. Recently, transgenic zebrafish were used as a live model to identify and track, via timelapse microscopy, macrophage subtypes expressing TNF- $\alpha$ [49]. Aseptic wounding and also infection with E. coli triggered macrophage recruitment, of which only a subset started to express TNF- $\alpha$ as well as some other pro-inflammatory cytokines, including IL- $1 \beta$ and IL-6. Of interest, fate tracing of TNF- $\alpha(+)$ macrophages during the time-course of inflammation demonstrated that these pro-inflammatory macrophages converted into other phenotypes. This study elegantly confirms that the diversity and plasticity typical of mammalian macrophages is also common to fish macrophages and may contribute to the ongoing discussion on the existence of one-directional transition states from a M2 to a M1 phenotype, or vice versa. 
One complicating factor in studies on the role of TNF- $\alpha$ is that most teleost fish species possess multiple isoforms [13, 50]. Functional studies with recombinant proteins have indicated conserved functions in goldfish and trout [51, 52] but also non-conserved functions of TNF- $\alpha$ in carp and seabream [53, 54]. So far, most studies have addressed two isoforms (TNF- $\alpha 1$ and TNF- $\alpha 2$ ), but recently a third isoform (TNF- $\alpha 3$ ) with only low identities to the other two TNF isoforms was described [55]. The new 'type II' TNF- $\alpha$ has only a short stalk suggesting it might function as a membrane form only, and could be especially interesting because it appears to have a low basal, but relatively early induced gene expression after LPS stimulation of macrophages. It is clear that studying TNF- $\alpha$ in relation to M1 polarization of fish macrophages is an area of research which requires further investigation of TNF- $\alpha$ isoforms while taking advantage of in vivo studies building on the availability of zebrafish transgenic for TNF- $\alpha$.

Granulocyte macrophage colony-stimulating factor (GM-CSF, CSF-2) is the latest addition to the M1 category of stimuli [6]. GM-CSF is a member of a discrete family of cytokines which also includes IL- 3 and IL- 5 which regulate the growth, differentiation, migration and effector function activities of many hematopoietic cells and leukocytes with receptors comprised of a cytokine-specific alpha chain $(C S F 2 R \alpha)$ and a shared beta chain (CSF2-R $\beta$ ) for signaling [1]. Although this cytokine family appears very poorly conserved [56], the existence of CSF2-R $\beta$ in fish genomes has been previously described [57]. Only recently, candidates for IL-3/ IL-5/GM-CSF family members have been identified in the elephant shark genome [58] and in genomes of some (cyprinid) fish species [10]. This recent identification of candidate GM-CSF-like cytokines in fish genomes opens the way for functional studies on activation of macrophages with recombinant GM-CSF to study the conservation of this form of activation of fish cells into macrophages with M1 profiles.

\section{M2 macrophages in fish}

M2 macrophages can be generally characterized as having 'anti-inflammatory' phenotypes, when developed in the presence of the $\mathrm{T}_{\mathrm{H}} 2$ cytokines IL-4 and/or IL-13 [1]. Typically, these M2 macrophages show increased arginase activity and produce proteins important for 'healing' processes, including the generation of extracellular matrix and polyamines (putrescine, spermidine, and spermine) important for cell growth and division. Whereas the enzyme iNOS is omnipresent 
in $\mathrm{M} 1$ and converts the amino acid L-arginine into L-citrulline and NO, M2 macrophages up-regulate arginase enzymatic activity to compete and convert the same substrate into L-ornithine and urea, counter-balancing the activity of M1. Here, it is not the intention to extensively discuss all different stimuli that can lead to the development of mammalian M2 macrophages but rather we will try and put in perspective the relevant data available for fish macrophages that fit M2 phenotypes. Overall, M2 macrophages can be grouped [6] to include i) macrophages activated by IL-4 or IL-13 [also named M2a: 3], ii) macrophages activated during parasite infection with protozoans or helminths either as a combination stimulus with apoptotic cells or immune complexes (also named M2b), iii) macrophages activated by macrophage colony stimulating factor CSF-1 but also iv) macrophages de-activated by glucocorticoids or by cytokines such as TGF- $\beta$ or IL-10 (also named M2c or regulatory macrophages).

Stimulation of macrophages with IL-4/-13 forms the best characterized antiinflammatory (M2) side of the balance in the M1/M2 paradigm. In mammals, IL-4 and IL-13 have overlapping but distinct roles in inflammatory responses to extracellular parasites via production of IgE, differentiation of $\mathrm{T}_{\mathrm{H}} 2$ lymphocytes and activation of M2 [59]. The heterodimeric receptor complex for IL-4 and IL-13 consists of a common receptor subunit (IL-4R $\alpha$ ), which is the molecular basis for their overlap in biological functions, and a receptor subunit specific for each cytokine [60]. The evolution of IL-4 and IL-13 and their receptor subunits has recently been reviewed [61]. IL-4 and IL-13 are found side by side in the mammalian genome. Given this genomic organization it seems likely that a single IL-4/13 ancestor gene existed in jawless vertebrates, which has been duplicated in different lineages by whole genome duplication and/or tandem duplication events [61]. In fish, at least two IL-4/ IL-13 genes exist (IL-4/13A and IL-4/13B), both with low homology to IL-4 and IL13 [62, 63], of which maybe IL-4/13A shows the most complete synteny with other genes in the $\mathrm{T}_{\mathrm{H}} 2$ cytokine complex [10]. This could fit the observation that zebrafish injected with recombinant IL-4/13A show increased numbers of DC-SIGN+ (CD209) cells and IgZ-2+ B cells in peripheral blood $[64,65]$. Recombinant zebrafish IL$4 / 13 \mathrm{~A}$ was shown to bind to the zebrafish IL- $4 \mathrm{R} \alpha$ chain [66]. Of interest, a $\mathrm{T}$ cell line (TCR+, CD4-1+) of common carp was established that expresses IL-4/13B, thus showing a $\mathrm{T}_{\mathrm{H}} 2$-like phenotype [67]. In vitro testing of supernatants of this cell line on common carp macrophages could verify the ability, or inability, of the second IL-4/13 isoform to drive macrophages to express an M2 phenotype. Without doubt, 
it would be interesting within the $\mathrm{T}_{\mathrm{H}} 2 / \mathrm{M} 2$ context, to study gene expression profiles of IL-4/13-stimulated fish macrophages for the presence of typical M2 markers, including arginase, to determine the degree of conservation in activation profile within the M2 group of macrophages found in a lower vertebrate.

Studies in mice have shown that arginase gene expression and activity can be induced by IL-13 via the increase of intracellular cAMP and tyrosine kinase phosphorylation. Exogenous cAMP can be used for a cytokine-independent activation of M2 macrophages based on the fact that cAMP-mediated activation of protein kinase A can mimic the effect of IL-13 as the primary signaling pathway responsible for the production of arginase [68]. Mouse macrophages isolated from the resolving (healing) phase of an acute peritonitis express an M2 phenotype controlled by cAMP and in particular, characterized by an increase in arginase-I and IL-10 and expression of the mannose receptor, the latter considered a typical M2 marker. Apparently, cAMP can control the balance of pro-inflammatory versus anti-inflammatory cytokines once signals that drive inflammation are dampened [69]. Human macrophages can also directly sense extracellular cAMP, inducing a phenotype resembling M2 macrophages. It is likely that a direct influx of cAMP into the cell or activation of adenylyl cyclases via adenosine receptors leads to an increase of intracellular second messenger cAMP, controlling the anti-inflammatory effects on monocytes [70]. Macrophages of common carp stimulated with exogenous cAMP increase arginase activity but not NO production, and the induced arginase activity could be specifically inhibited by the NOS inhibitor N(omega)-hydroxynor-L-arginine (NOHA) [14]. Therefore, common carp macrophages show the intrinsic capability to polarize into M2-type/healing state as shown by high levels of arginase.

Susceptibility to both protozoan and helminth infections have been linked to the presence of M2 macrophages [71-73]. Exactly which (sub) types develop in vivo depends on parasite virulence, host genotype and infection stage, but also possible co-infections with other parasites such as helminths that can be co-endemic with protozoan parasites [72]. For fish, there are a number of studies that have measured arginase gene expression during bacterial or parasitic infection [16], but gene expression studies based on whole organ transcription analysis cannot be more than indicative for the presence of either M1 or M2, although maybe the use of an iNOS/arginase gene expression ratio [74] might be considered an interesting 
approach to quantify macrophage polarization in vivo. Atlantic salmon (Salmo salar) infected with salmon louse show an inflammatory pattern of gene expression in damaged skin with signs of $\mathrm{T}_{\mathrm{H}} 2$-like responses. Of interest, up-regulation of arginase-1, among others, was seen in the intact skin of infected fish and of the major components of extracellular matrix, TGF- $\beta$ and IL-10, but only at the adult stage of sea louse infection [75]. Taken together with up-regulation of matrix metalloproteinases (MMP), this classifies the wounds afflicted by sea louse as chronic and possibly presents an interesting infection model to study iNOS/ arginase gene expression ratio and thus M1/M2 polarization in salmon.

Few studies have addressed macrophage polarization in vivo; some indications exist from our studies on experimental infections of common carp with either of two related Kinetoplastid protozoan parasites. Infections with Trypanoplasma borreli appear characterized by the presence of large numbers of M1 macrophages as deduced from an increase in IFN- $\gamma$, TNF- $\alpha$ and iNOS gene expression and associated increase in serum nitrite levels and tissue nitration [76-82]. Experimental infections with Trypanosoma carassii, in contrast, do not result in a prominent NO response $[77,83]$ but instead result in elevated levels of arginase enzyme activity, particularly during the later phase of infection [78]. Of interest, ex vivo studies with head kidney leukocytes from T. borreli-infected carp, re-stimulated with LPS showed significant amounts of NO production, whereas re-stimulation of head kidney leukocytes from T. carassii-infected carp with cAMP show higher levels of arginase, but not vice versa [78]. This suggests that macrophages from $T$. borreli-infected carp could be polarized toward an M1 phenotype, whereas macrophages from T. carassiiinfected could be polarized toward an M2 phenotype. Overall, it is clear that more experimental studies with parasite-infected fish are required to characterize in vivo activation of M2 in fish.

Macrophage colony-stimulating factor (M-CSF, CSF-1) is the latest addition to the M2 category of stimuli [6]. M-CSF is the primary regulator of proliferation, differentiation, and survival of macrophages via its cognate colony-stimulating factor-1 receptor (CSF1-R) [reviewed in 84], which leads to activation of STAT6 and other regulators, many of which are also part of the IL-4/-13 signaling pathways. M-CSF appears relatively well-conserved, although rainbow trout, zebrafish and goldfish CSF-1 each have a unique exon/intron structure [85]. Signaling through CSF1-R mediates the proliferation, differentiation, and activation of macrophages 
and their progenitors. In goldfish, CSF1-R is expressed on all macrophage sub-populations (progenitors, monocytes, macrophages), and increases with macrophage development [86]. Recombinant trout CSF-1 promotes the growth of head kidney leukocytes, and up-regulates the expression of CXCR-3 in macrophages, suggesting a role in the trafficking of macrophages to sites of inflammation or injury [85]. Recombinant goldfish CSF-1 induces the differentiation of monocytes into macrophages and proliferation of monocyte-like cells with pro-inflammatory effects on these cell types [43]. The latter could be abrogated by anti-CSF1-R antibody and also by a soluble CSF1-R, which presence appears to be a mechanism for the regulation of CSF-1 function seemingly unique to fish [87-89]. Soluble CSF1-R modulates reactive oxygen production, nitric oxide synthesis, chemotaxis, and phagocytosis in cultured macrophages and plays a role in the control of inflammation in vivo, which includes modulation of macrophage function and recruitment [90, 91]. Although there is as yet no clear evidence that soluble CSF1-R plays a role in determining fish M2 macrophage phenotypes, it might be interesting to further investigate soluble CSF1-R in the context of macrophage polarization.

Macrophages de-activated by glucocorticoids or by the cytokines TGF- $\beta$ or IL10 can also be included in the group of M2(c) macrophages. In lower animals including snails, the expression of arginase can be induced by the presence of parasite antigens and TGF- $\beta$ leading to collagen synthesis, without the presence of $\mathrm{T}_{\mathrm{H}} 2$ cytokines [92]. In fact, it has been suggested that TGF- $\beta$-signaling, rather than microbial stimuli, would be the evolutionarily oldest trigger to initiate M1/M2 macrophage polarization [92]. Of the large TGF- $\beta$ superfamily, TGF- $\beta 1$ seems to be the most potent regulator of iNOS [93]. The extremely high degree of conservation of TGF- $\beta 1$ [94], suggests that its signaling function in inflammatory responses and tissue necrosis may have been preserved over time [92]. Recombinant grass carp IL-10 and recombinant grass carp TGF- $\beta 1$ both attenuate LPS-stimulated inflammatory gene expression in monocytes/macrophages [95]. Recombinant goldfish IL-10 reduces radical production in monocytes and transcription of specific pro-inflammatory cytokines [96]. Recombinant common carp IL-10 deactivates both neutrophils and macrophages, as shown by inhibition of oxygen and nitrogen radical production as well as reduced expression of pro-inflammatory genes, but also MHC genes involved in antigen presentation. Similar to mammalian IL10, carp IL-10 acts through a signaling pathway involving phosphorylation of STAT-3, ultimately leading to early up-regulation of SOCS-3 expression [97]. It would be of 
interest to study more extensively the gene expression profiles of TGF- $\beta 1$ - or IL- 10 stimulated fish macrophages in order to examine the degree of conservation in the (de)activation profiles and effects on expression of M2 markers of fish macrophages.

\section{The relevance of catabolizing L-arginine}

Arginine is a non-essential or conditionally-essential amino acid in mammals but a truly essential amino acid in birds and fish, because the latter animal species cannot synthesize arginine de novo. In mammals, uptake in the small intestine appears to be the major route of citrulline for de novo synthesis of arginine by the kidneys in mammals, resulting in normal plasma levels of arginine in humans of 100-200 $\mu \mathrm{M}$ [98]. Uptake of citrulline in the small intestine usually is sufficient for a healthy adult, but the biosynthetic pathway may not always produce enough arginine during growth or disease [99], in which cases arginine supplementation is also required in humans. Animal feed for birds and fish always need to contain sufficient arginine to maintain a healthy diet. In catfish (Ictalurus punctatus), healthy plasma levels are around 100-200 $\mu \mathrm{M}$ [100]. Recent experiments indicated that higher levels of dietary arginine could attenuate the inflammatory response induced by LPS in both, chicken [101] and teleosts, e.g. Chinese common carp [102]. Given the fact that teleosts cannot synthesize arginine de novo, nutritional supplementation can possibly open a route to influence arginine concentrations at the whole animal level, and thereby influence macrophage polarization and fish health.

In general, arginine is preferentially taken up via members of the solute carrier family 7 (SLC7) [103]. Cationic amino acid transporter-1 (CAT-1), or SLC7-A1, is one of the main transporters for arginine uptake into cells, where arginine is subsequently used for protein synthesis. Murine macrophages constitutively express high affinity SLC7-A1 (CAT-1) but transport arginine via the low affinity SLC7-A2 (CAT-2) transporter when activated [99]. Grass carp SLC7-1 expression is down-regulated in fasting animals [104], but receptor expression in macrophages was not checked. Clearly, identification and characterization of members of the SLC7 family members relevant to fish macrophages requires further investigation.

Upon arginine transport into cells, mammalian cells can catabolize arginine by four classes of enzymes: nitric oxide synthase (NOS), arginase, arginine decarboxylase (ADC), and arginine:glycine aminotransferase (AGAT) [105]. In macrophages, the enzymes NOS and arginase play a major role in arginine catabolism and 
thus a central role in the polarization of immune responses and physiology of macrophages associated with NOS (M1) and arginase (M2) activity.

\section{Inducible nitric oxide synthase (iNOS) as a marker for M1}

Most probably, multiple events of parallel evolution of NOS exist which has led to different lineages, with inducible NOS (iNOS) as the most basal ancestral prototype. In invertebrates, nitric oxide acts as a signaling molecule and does not appear to have the cytotoxic function found in vertebrates [18]. Mammalian vertebrates have three isoforms of NOS: NOS1 or neuronal NOS (nNOS), NOS2 or iNOS, and NOS3 or endothelial NOS (eNOS). Both nNOS and eNOS are constitutively expressed enzymes that are calcium-dependent. In contrast, iNOS is inducible and independent of calcium because calmodulin binds to iNOS even at low intracellular calcium concentrations [18], an effect that probably occurs also in fish [76]. Most likely, eNOS originated as the last isoform within the mammalian clade and indeed, eNOS sequences appear absent from teleost fish genomes. NOS catabolizes arginine to nitric oxide and citrulline. M1 macrophages abundantly express the enzyme iNOS, but also express a pathway to catabolize citrulline via the citrulline-NO cycle for resynthesis of arginine, which allows these macrophages to ensure sufficient supply of arginine for prolonged NO synthesis [18]. Simultaneous generation of NO radicals and reactive oxygen species, including the radical superoxide $\left(\mathrm{O}_{2}^{-}\right)$can, for example, lead to the formation of peroxynitrite ( $\left.\mathrm{ONOO}^{-}\right)$, a powerful oxidant leading to the formation of nitrotyrosine [106]. Tyrosine nitration can be considered a hallmark of tissue injury and has been used as a specific (bio)marker for nitrosative stress linked with inflammation, a phenomenon also observed in fish [79, 107]. The combined generation of reactive nitrogen and reactive oxygen species helps define M1 as 'kill/fight type' of macrophages with iNOS (NOS-2) being an excellent marker for M1 macrophages of fish.

Teleosts are believed to have gone through three rounds (3R) of whole genome duplications, approximately 360 million years ago (MYA) [108]. Some, like the Salmonidae are assumed to have gone through an additional fourth round of duplication (4R) [109]. Likewise, within the Cyprinidae, common carp, but not zebrafish, have gone through an additional duplication round (4R). Indeed, the majority of exons of zebrafish have two hits in the carp genome [110]. Common carp and zebrafish are estimated to have diverged approximately 120 MYA [111], but have retained extraordinary levels of synteny [110]. Whole genome duplications 
can result in both gene loss and gene gain but there exist significant differences in gene retention for different functional categories of genes between fishes and land vertebrates [112]. As a result of 3R duplications zebrafish can express two copies where mammalian vertebrates express a single copy; where human and mouse express a single copy of inducible NOS, zebrafish express two copies of this gene (NOS-2A and NOS-2B). Syntenic analysis shows conserved synteny for NOS-2B, but not NOS-2A, suggestive of gene divergence of NOS-2 genes in zebrafish [113]. Although most research in zebrafish has focused on NOS-2A [114, 115], NOS-2B appears functionally conserved [116, 117]. Building on our well-characterized in vitro culture system to polarize common carp macrophages into cells with M1 phenotypes, while using the zebrafish and common carp genomes for gene annotation, we collected these M1 macrophages for RNA analysis and subsequent transcriptome analysis. Head kidney-derived macrophages, when stimulated with

RNA-Seq analysis of NOS-2A

\begin{tabular}{|c|c|c|c|c|}
\hline \multirow{2}{*}{ Stimulus } & \multicolumn{2}{|c|}{ Normalized counts } & \multirow{2}{*}{ Fold change } & \multirow{2}{*}{ padj } \\
\hline & Control & Stimulated & & \\
\hline cAMP & 0.4 & 0 & 0 & 1 \\
\hline LPS & 2.3 & 1.0 & 0.5 & 1 \\
\hline
\end{tabular}

\section{Phylogenetic analysis of NOS-1 and NOS-2}

RNA-Seq analysis of NOS-2B

\begin{tabular}{|c|c|c|c|c|}
\hline \multirow{2}{*}{ Stimulus } & \multicolumn{2}{|c|}{ Normalized counts } & \multirow{2}{*}{ Fold change } & \multirow{2}{*}{ padj } \\
\hline & Control & Stimulated & & \\
\hline cAMP & $4412(1835-6988)$ & $3978(1264-6692)$ & $0.8(0.7-1)$ & $0.2-1$ \\
\hline LPS & 2076 (776-3377) & $98688(34485-162891)$ & $46(44-48)$ & $4.21 \mathrm{E}-60-1.14 \mathrm{E}-57$ \\
\hline
\end{tabular}

qPCR analysis of NOS-2B
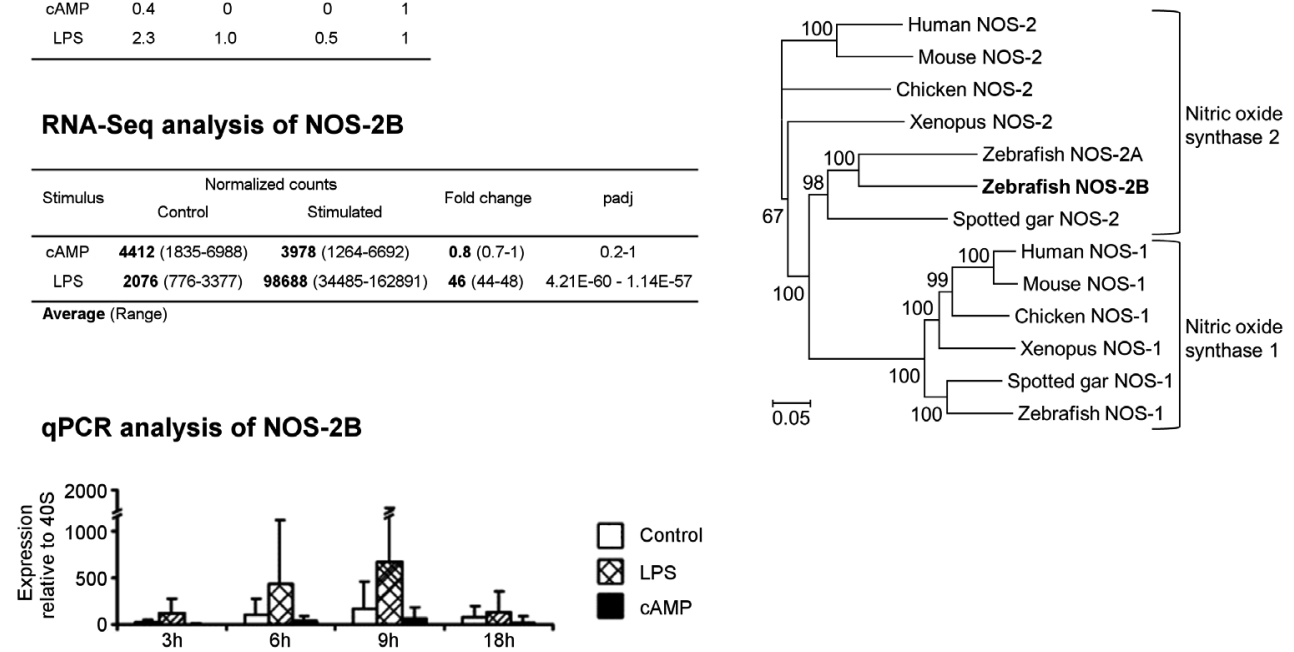

Figure 2.2 Nitric oxide synthase-2(B) is a marker for M1 macrophages of fish

Common carp head kidney-derived macrophages were stimulated with LPS, or cAMP, and gene expression analyzed by RNAseq (Illumina). Nitric oxide synthase 2 (NOS2) has two hits in the zebrafish genome (NOS-2A and NOS-2B) because of a genome duplication event (3R), whereas common carp have two hits for NOS-2A and $-2 \mathrm{~B}$ each in the genome (and transcriptome) because of an additional duplication event (4R). Only NOS-2B, but not NOS-2A is expressed in common carp macrophages. Accordingly, gene expression of NOS-2B was studied by real-time qPCR using gene-specific primers and found highly up-regulated only after stimulation with LPS. 
LPS, polarized into M1 phenotypes characterized by a high up-regulation of NOS$2 \mathrm{~B}$, but not NOS-2A gene expression (Fig. 2.2). This observation is supported by the fact that phylogenetic and syntenic analyses indicate that NOS-2B, but not NOS-2A, is associated with syntenic genes identified for NOS-2 genes in higher vertebrates [113], suggesting that NOS-2B might be a robust marker for M1 macrophages of (cyprinid) fish.

\section{Arginase as a marker for $\mathrm{M} 2$}

The enzyme arginase is present in bacteria, yeasts, plants, invertebrates, and vertebrates, although phylogenetically, plant arginases are closer to the bacterial agmatinases, which pre-date arginase evolutionary. Arginases from plants and ammoniotelic animals, organisms which excrete soluble ammonia, are localized in mitochondria (Jenkinson et al., 1996). The primordial function of arginase appears to be regulation of cellular arginine and ornithine catabolism, unrelated to the urea cycle, to deliver ornithine for processes of extracellular matrix synthesis and polyamine synthesis important for organogenesis and wound healing [92]. Most eukaryotic cells have a polyamine transporter system on their cell membrane that facilitates the internalization of exogenous polyamines; a system highly active in rapidly proliferating cells [118]. The generation of arginase activity and downstream production of collagen and polyamines help define M2 as a 'healing type' of macrophage with arginase being a robust marker for M2 macrophages of fish.

Vertebrates have a relatively recent arginase gene duplication that, at least in mammals, has led to a cytosolic and a mitochondrial form, named ARG-1 and ARG-2, respectively. Although both isoforms catabolize arginine, they are encoded by two different genes which differ in cellular expression and cell-type-specific regulation [119]. In ureotelic animals; organisms which excrete excess nitrogen as urea (e.g. mammals), ARG-1 is expressed primarily in the liver and is a cytoplasmic enzyme central to the hepatic urea cycle, whereas ARG-2 is a mitochondrial enzyme that is expressed in almost all organs. Like other vertebrates, teleost fish express ARG-1 and ARG-2, but the presence of mitochondrial targeting sequences in both isoforms [14] suggests that these enzymes are both of the mitochondrial form. Apparently, arginases function as mitochondrial enzymes in fish, which generally are ammoniotelic (exceptions are marine elasmobranchs, coelacanths and African 
RNA-Seq analysis of ARG-2

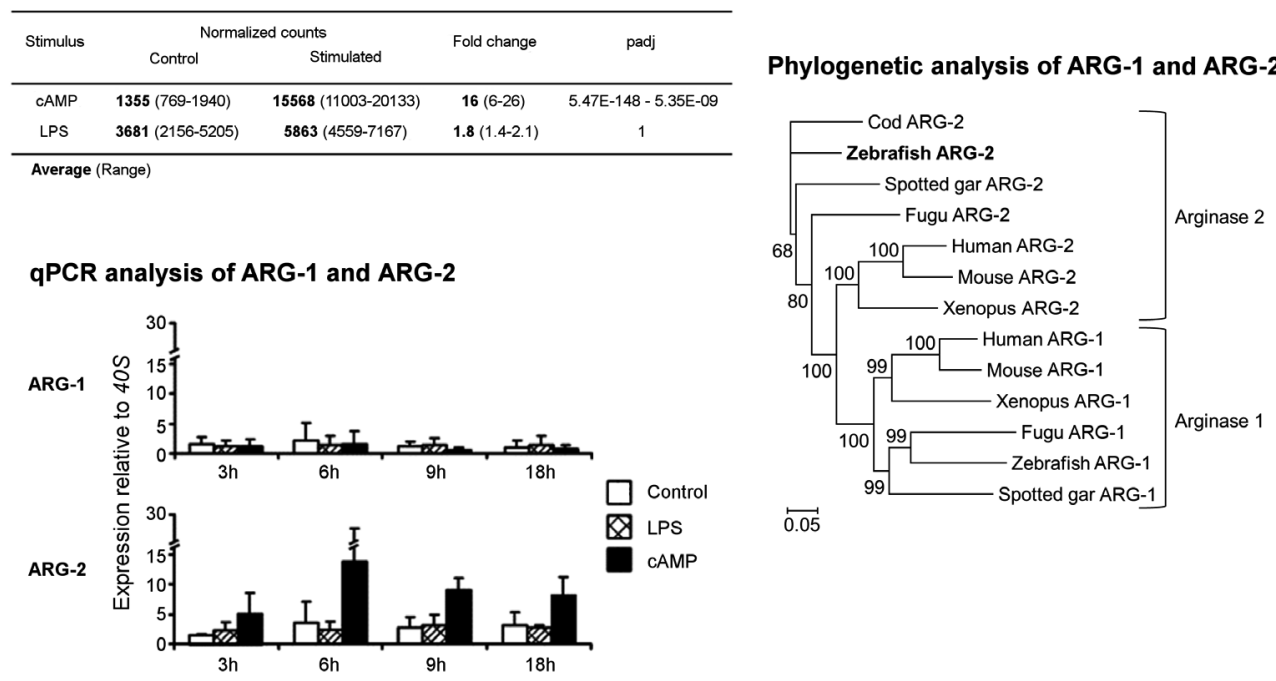

Figure 2.3 Arginase-2 is a marker for fish M2 macrophages of fish

Common carp head kidney-derived macrophages were stimulated with LPS, or cAMP, and gene expression analyzed by RNAseq (Illumina). Zebrafish arginase (here, ARG-2) has two hits in the common carp genome (and transcriptome) because of an additional genome duplication event (4R). Only ARG2 , but not ARG-1 is expressed in common carp macrophages. Accordingly, gene expression of ARG-2 was studied by real-time qPCR using gene-specific primers and found highly up-regulated only after stimulation with cAMP.

lungfishes) and thus do not excrete urea through the kidneys but ammonia mainly through the gills.

Mammalian M2 macrophages express both ARG-1 and ARG-2, but numerous studies have shown that it is the enhanced expression of the cytosolic ARG-1 that depletes these macrophages of intracellular arginine pools leaving less substrate for iNOS [18] and thus counteracts the development of M1 macrophages and leads to the production of ornithine and downstream synthesis of several polyamines, characteristic of M2 macrophages. Head kidney-derived macrophages of common carp, when stimulated with cAMP, polarize into M2 phenotypes characterized by a high up-regulation of ARG-2, but not ARG-1 gene expression (Fig. 2.3), suggesting that ARG-2 might be an excellent marker for M2 macrophages of fish. 


\section{Future developments}

A major limitation of understanding M1/M2 polarization in murine and human models is the lack of in vivo imaging potential. Fish models, such as the zebrafish and medaka (Oryzias latipes) have led the way in this respect, with intra-vital, in vivo imaging of immune processes in their embryos being a major advantage of the model due to their small size and transparent tissues [120, 121]. The zebrafish, especially, has emerged as a useful model for the study of inflammation and infection, with a number of well-characterised models to investigate both processes [122,123]. Zebrafish embryos have functional macrophages at 1 day postfertilization (dpf) and neutrophils by $2 \mathrm{dpf}$. The development of fluorescent reporter lines marking these cell types enables detailed analysis during inflammatory and infection processes [124-126]. The relative ease of genetic manipulation is a major advantage of the zebrafish model, and the genes for fluorescent proteins (e.g. green fluorescent protein, GFP) can be inserted into the genome using bacterial artificial chromosome (BAC) recombineering, or can be placed under the control of a partial promoter, to give specific expression in the cell type of interest [126, 127]. The rapidly-increasing number of well-characterized experimental infection and inflammation models in zebrafish are highly valuable for in vivo investigations of (anti) inflammatory processes.

The complex behaviors of zebrafish macrophage/neutrophil sub-populations observed during inflammation and infection have shown that M1- and M2-like macrophage subsets may indeed also be present in the zebrafish embryo/larvae [124, $128,129]$. Given the in vitro findings in (for example) the closely-related common carp this may not be surprising, but these potential subsets have yet to be fully identified and characterized in vivo. The zebrafish has already emerged as being a particularly useful animal model for the study of inflammation and infection in vivo and thus zebrafish may help to examine macrophage polarization, particularly the plasticity of macrophages to switch subtypes during an inflammation/ infection response, which so far has proven difficult to observe in vivo. Since the current classification of M1/classically-activated and M2/alternatively-activated macrophages have, in the main, arisen from in vitro studies using specific stimuli, zebrafish may help with truly reflecting the complex nature of in vivo environments. Zebrafish models of leukocyte biology are, in general, used from 2 to $5 \mathrm{dpf}$, while the optical properties of the embryo are optimal and before the onset of functional adaptive immunity. These properties make them an exciting model for 
investigation of the 'macrophage first' hypothesis, while not negating looking later in development when the adaptive immune response is active.

Much of the recent focus in zebrafish has been around TNF- $\alpha$, as an important cytokine involved in the activation of leukocytes after inflammation/infection challenge and a putative although not unique marker for M1. In a wellcharacterized zebrafish Mycobacterium marinum infection model of tuberculosis, TNF- $\alpha$ is up-regulated in infected macrophages and leads to production of reactive oxygen species to control the infection [53]. In the M. marinum model it has been shown that reactive nitrogen species mediated by NOS-2 aids infection control when stimulated by upregulated hypoxia signaling (via the hypoxia inducible factor-1 alpha, HIF-1 $\alpha$, transcription factor), evidence that suggests that NOS-2 may have a role in leukocyte subset behavior during zebrafish infection [107, 115]. The possibility of developing M1 (e.g., NOS-2) and M2 (e.g., ARG-2) zebrafish transgenic lines using different fluorescent markers is an exciting prospect, as these could be combined in double or triple transgenics with existing marker lines to generate a model in which the plasticity of macrophages over the course of an infection/inflammatory process could be studied in vivo. Their use in well-defined infections, such as the M. marinum model, would allow in detail characterisation of macrophage phenotype over the timecourse of an infection, and importantly, realtime observation of one-directional switching events in vivo at the level of individual cells. Crucially, the genetic tractability in the zebrafish embryo model would allow molecular dissection of the pathways involved in any M2 to M1 (or vice versa) switching events. In order for transgenic M1 and M2 reporter lines to be generated a set of reliable, and preferentially evolutionary-conserved, markers for these polarized cell types are required. In a recent experiment, building on a well-characterized in vitro culture system for head kidney-derived macrophages, we made use of the large size of common carp to collect polarized macrophage subtypes for transcriptome analysis using the largely syntenic zebrafish and common carp genomes for annotation. As previously discussed, microbial ligand LPS polarized these macrophages into M1, whereas extracellular cAMP polarized these macrophages into M2, with NOS-2(B) and ARG-2 as respective markers, each with low $\mathrm{P}$ value and high fold-change of gene expression. Of course, these preliminary analyses require confirmation and, preferably, expansion with other stimuli such as IFN- $\gamma$ and IL-4/IL-13. However, the value of the current dataset may be apparent 
from the confirmation of NOS-2 as marker for M1 and ARG-2 as marker for M2 macrophages of fish.

In addition (data not shown), un-stimulated head kidney-derived carp macrophages express at medium-high levels (1000-4000 reads per million) the beta chain shared by the GM-CSF cytokine family and also the common receptor subunit IL-4R $\alpha$ for recognition of IL-4/-13. Expression levels of IL-4R $\alpha$ show a 5-fold up-regulation but only after cAMP stimulation, suggesting cAMP indeed induces a M2-like phenotype in these macrophages. Furthermore, MRC-1B, provisionally annotated as the mannose receptor, has a high basal expression (5,000-25,000 reads per million) in un-stimulated macrophages, shows a 5-fold down-regulation in LPS-stimulated cells but a 3-fold up-regulation in cAMP-stimulated cells. The latter data on MRC$1 \mathrm{~B}$ suggest that indeed this could be the mannose receptor and a suitable M2 marker for fish macrophages. It is our expectation that although NOS-2 and ARG-2 may prove good markers for M1 and M2 macrophages, respectively, the expression of these molecules may not always be exclusive for these macrophage phenotypes and additional markers (e.g., IL-4R $\alpha$ or MRC1-B for M2) may be required to confirm profiles of polarized macrophages. In zebrafish, the availability of wellcharacterised macrophage fluorescent transgenic lines, described earlier (MPEG1 and FMS driven expression), in combination with novel M1/M2 reporter lines, will allow identification of NOS-2 and ARG-2 positive macrophages even if other cell types also express these genes. Our preliminary findings indicate the value of combining in vitro culture systems of polarized head kidney-derived macrophages with the genetic identification of M1 and M2 markers for fish that can subsequently be used in zebrafish to study polarized immune responses in vivo.

\section{Acknowledgements}

Research leading to this review was funded by the European Commission under the $7^{\text {th }}$ (FP7) and $8^{\text {th }}$ (H2020) Framework Programs for Research and Technological Development of the European Union (NEMO PITN-GA-2008-214505, TARGETFISH Grant No. 311993 and PARAFISHCONTROL Grant No. 634429). 


\section{References}

1. Martinez FO, Helming L, and Gordon S (2009) Alternative Activation of Macrophages: An Immunologic Functional Perspective. Annu Rev Immunol 27:451-483. doi: 10.1146/annurev. immunol.021908.132532

2. Mills CD, Kincaid K, Alt JM, Heilman MJ, and Hill AM (2000) M-1/M-2 Macrophages and the Th1/Th2 Paradigm. J Immunol 164:6166-6173. doi: 10.4049/jimmunol.164.12.6166

3. Mantovani A, Sica A, Sozzani S, Allavena P, Vecchi A, and Locati M (2004) The chemokine system in diverse forms of macrophage activation and polarization. Trends Immunol 25:677686. doi: 10.1016/j.it.2004.09.015.

4. Xue J, Schmidt SV, Sander J, Draffehn A, Krebs W, Quester I, De Nardo D, Gohel TD, Emde M, Schmidleithner L, Ganesan H, Nino-Castro A, Mallmann MR, Labzin L, Theis H, Kraut M, Beyer M, Latz E, Freeman TC, Ulas T, and Schultze JL (2014) Transcriptome-based network analysis reveals a spectrum model of human macrophage activation. Immunity 40:274-88. doi: 10.1016/j. immuni.2014.01.006.

5. Mills CD and Ley K (2014) M1 and M2 Macrophages: The Chicken and the Egg of Immunity. J Innate Immun 6:716-726. doi: 10.1159/000364945.

6. Murray PJ, Allen JE, Biswas SK, Fisher EA, Gilroy DW, Goerdt S, Gordon S, Hamilton JA, Ivashkiv LB, Lawrence T, Locati M, Mantovani A, Martinez FO, Mege J-L, Mosser DM, Natoli G, Saeij JP, Schultze JL, Shirey KA, Sica A, Suttles J, Udalova I, van Ginderachter JA, Vogel SN, and Wynn TA (2014) Macrophage Activation and Polarization: Nomenclature and Experimental Guidelines. Immunity 41:339-340. doi: 10.1016/j.immuni.2014.07.009.

7. Martinez FO and Gordon S (2014) The M1 and M2 paradigm of macrophage activation: time for reassessment. F1000Prime Rep 6:13. doi: 10.12703/P6-13.

8. Ward AE and Rosenthal BM (2014) Evolutionary responses of innate immunity to adaptive immunity. Infect Genet Evol 21:492-496. doi: 10.1016/j.meegid.2013.12.021.

9. Magor BG and Magor KE (2001) Evolution of effectors and receptors of innate immunity. Dev Comp Immunol 8-25:651-682. doi: 10.1016/S0145-305X(01)00029-5.

10. Yamaguchi T, Takizawa F, Fischer U, and Dijkstra JM (submitted 2015) Along the axis between type 1 and type 2 immunity; principles conserved in evolution from fish to mammals. Biology 4:814-859. doi: 10.3390/biology4040814.

11. Bird S, Zou J, and Secombes CJ (2006) Advances in Fish Cytokine Biology Give Clues to the Evolution of a Complex Network. Curr Pharm Des 12:3051-3069. doi: $10.2174 / 138161206777947434$.

12. Secombes CJ, Wang T, and Bird S (2011) The interleukins of fish. Dev Comp Immunol 35:13361345. doi: 10.1016/j.dci.2011.05.001.

13. Wang T and Secombes CJ (2013) The cytokine networks of adaptive immunity in fish. Fish Shellfish Immunol 35:1703-1718. doi: 10.1016/j.fsi.2013.08.030.

14. Joerink M, Savelkoul HFJ, and Wiegertjes GF (2006) Evolutionary conservation of alternative activation of macrophages: Structural and functional characterization of arginase 1 and 2 in carp (Cyprinus carpio L.). Mol Immunol 34:1116-1128. doi: 10.1016/j.molimm.2005.07.022. 
15. Joerink M, Ribeiro CMS, Stet RJM, Hermsen T, Savelkoul HFJ, and Wiegertjes GF (2006) Head Kidney-Derived Macrophages of Common Carp (Cyprinus carpio L.) Show Plasticity and Functional Polarization upon Differential Stimulation. J Immunol 177:61-69. doi: 10.4049/ jimmunol.177.1.61.

16. Forlenza M, Fink IR, Raes G, and Wiegertjes GF (2011) Heterogeneity of macrophage activation in fish. Dev Comp Immunol 35:1246-1255. doi: 10.1016/j.dci.2011.03.008.

17. Thomas AC and Mattila JT (2014) "Of Mice and Men”: Arginine Metabolism in Macrophages. Front Immunol 5:479. doi: 10.3389/fimmu.2014.00479.

18. Rath M, Müller I, Kropf P, Closs EI, and Munder M (2014) Metabolism via Arginase or Nitric Oxide Synthase: Two Competing Arginine Pathways in Macrophages. Front Immunol 5:532. doi: 10.3389/fimmu.2014.00532.

19. Gordon S (2003) Alternative activation of macrophages. Nat Rev Immunol 3:23-35. doi: 10.1038/ nri978.

20. Taylor PR, Martinez-Pomares L, Stacey M, Lin HH, Brown GD, and Gordon S (2004) Macrophage receptors and immune recognition. Annu Rev Immunol 23:901-944. doi: 10.1146/annurev. immunol.23.021704.115816.

21. Palti Y (2011) Toll-like receptors in bony fish: From genomics to function. Dev Comp Immunol 35:1263-1272. doi: 10.1016/j.dci.2011.03.006.

22. Boltaña S, Roher N, Goetz FW, and MacKenzie SA (2011) PAMPs, PRRs and the genomics of gram negative bacterial recognition in fish. Dev Comp Immunol 35:1195-1203. doi: 10.1016/j. dci.2011.02.010.

23. Hansen JD, Vojtech LN, and Laing KJ (2011) Sensing disease and danger: A survey of vertebrate PRRs and their origins. Dev Comp Immunol 35:886-897. doi: 10.1016/j.dci.2011.01.008.

24. Rauta PR, Samanta M, Dash HR, Nayak B, and Das S (2014) Toll-like receptors (TLRs) in aquatic animals: Signaling pathways, expressions and immune responses. 158:14-24. doi: 10.1016/j. imlet.2013.11.013.

25. Aoki T, Hikima J-i, Hwang SD, and Jung TS (2013) Innate immunity of finfish: Primordial conservation and function of viral RNA sensors in teleosts. Fish Shellfish Immunol 35:16891702. doi: 10.1016/j.fsi.2013.02.005.

26. Zhang J, Kong X, Zhou C, Li L, Nie G, and Li X (2014) Toll-like receptor recognition of bacteria in fish: Ligand specificity and signal pathways. Fish Shellfish Immunol 41:380-388. doi: 10.1016/j. fsi.2014.09.022.

27. Pietretti D and Wiegertjes GF (2014) Ligand specificities of Toll-like receptors in fish: Indications from infection studies. Dev Comp Immunol 43:205-222. doi: 10.1016/j.dci.2013.08.010.

28. Kanwal Z, Wiegertjes GF, Veneman WJ, Meijer AH, and Spaink HP (2014) Comparative studies of Toll-like receptor signalling using zebrafish. Dev Comp Immunol 46:35-52. doi: 10.1016/j. dci.2014.02.003.

29. Poynter S, Lisser G, Monjo A, and DeWitte-Orr S (2015) Sensors of Infection: Viral Nucleic Acid PRRs in Fish. Biology 4:460. doi: 10.3390/biology4030460. 
30. Sepulcre MP, Alcaraz-Pérez F, López-Muñoz A, Roca FJ, Meseguer J, Cayuela ML, and Mulero V (2009) Evolution of Lipopolysaccharide (LPS) Recognition and Signaling: Fish TLR4 Does Not Recognize LPS and Negatively Regulates NF-кB Activation. J Immunol 182:1836-1845. doi: 10.4049/jimmunol.0801755.

31. Sullivan C, Charette J, Catchen J, Lage CR, Giasson G, Postlethwait JH, Millard PJ, and Kim CH (2009) The Gene History of Zebrafish tlr4a and tlr4b Is Predictive of Their Divergent Functions. J Immunol 183:5896-5908. doi: 10.4049/jimmunol.0803285.

32. MacKenzie SA, Roher N, Boltaña S, and Goetz FW (2010) Peptidoglycan, not endotoxin, is the key mediator of cytokine gene expression induced in rainbow trout macrophages by crude LPS. Mol Immunol 47:1450-1457. doi: 10.1016/j.molimm.2010.02.009.

33. Hume DA (2015) The Many Alternative Faces of Macrophage Activation. Front Immunol 6:370. doi: 10.3389/fimmu.2015.00370.

34. Rieger AM and Barreda DR (2011) Antimicrobial mechanisms of fish leukocytes. Dev Comp Immunol 35:1238-1245. doi: 10.1016/j.dci.2011.03.009.

35. MMartinez FO, Gordon S, Locati M, and Mantovani A (2006) Transcriptional Profiling of the Human Monocyte-to-Macrophage Differentiation and Polarization: New Molecules and Patterns of Gene Expression. J Immunol 177:7303-7311. doi: 10.4049/jimmunol.177.10.7303.

36. Nau GJ, Richmond JFL, Schlesinger A, Jennings EG, Lander ES, and Young RA (2002) Human Macrophage Activation Programs Induced by Bacterial Pathogens. Proc Natl Acad Sci USA 99:1503-1508. doi: 10.2307/3057791.

37. Lowenstein CJ, Alley EW, Raval P, Snowman AM, Snyder SH, Russell SW, and Murphy WJ (1993) Macrophage nitric oxide synthase gene: two upstream regions mediate induction by interferon gamma and lipopolysaccharide. Proc Natl Acad Sci USA 99:9730-9734. doi: 10.1073/ pnas.90.20.9730.

38. Xie QW, Whisnant R, and Nathan C (1993) Promoter of the mouse gene encoding calciumindependent nitric oxide synthase confers inducibility by interferon gamma and bacterial lipopolysaccharide. The J Exp Med 177:1779-1784. doi: 10.1084/jem.177.6.1779.

39. Zou J and Secombes CJ (2011) Teleost fish interferons and their role in immunity. Dev Comp Immunol 35:1376-1387. doi: 10.1016/j.dci.2011.07.001.

40. Yang K, Zhang S, Chen D, Zhang A, Wang X, and Zhou H (2013) IFN-gamma-activated lymphocytes boost nitric oxide production in grass carp monocytes/macrophages. Fish Shellfish Immunol 35:1635-41. doi: 10.1016/j.fsi.2013.09.017.

41. Arts JAJ, Tijhaar EJ, Chadzinska M, Savelkoul HFJ, and Verburg-van Kemenade BML (2010) Functional analysis of carp interferon- $\gamma$ : Evolutionary conservation of classical phagocyte activation. Fish Shellfish Immunol 29:793-802. doi: 10.1016/j.fsi.2010.07.010.

42. Skjesol A, Liebe T, Iliev DB, Thomassen EI, Tollersrud LG, Sobhkhez M, Lindenskov Joensen L, Secombes CJ, and Jorgensen JB (2014) Functional conservation of suppressors of cytokine signaling proteins between teleosts and mammals: Atlantic salmon SOCS1 binds to JAK/STAT family members and suppresses type I and II IFN signaling. Dev Comp Immunol 45:177-89. doi: 10.1016/j.dci.2014.02.009. 
43. Grayfer L, Hanington PC, and Belosevic M (2009) Macrophage colony-stimulating factor (CSF-1) induces pro-inflammatory gene expression and enhances antimicrobial responses of goldfish (Carassius auratus L.) macrophages. Fish Shellfish Immunol 26:406-413. doi: 10.1016/j. fsi.2008.12.001.

44. Grayfer L, Garcia EG, and Belosevic M (2010) Comparison of Macrophage Antimicrobial Responses Induced by Type II Interferons of the Goldfish (Carassius auratus L.). J Biol Chem 31:23537-23547. doi: 10.1074/jbc.M109.096925.

45. Sieger D, Stein C, Neifer D, van der Sar AM, and Leptin M (2009) The role of gamma interferon in innate immunity in the zebrafish embryo. Dis Model Mech 2:571-581. doi: 10.1242/ dmm.003509.

46. Shibasaki Y, Yabu T, Araki K, Mano N, Shiba H, Moritomo T, and Nakanishi T (2014) Peculiar monomeric interferon gammas, IFN $\gamma$ rel 1 and IFN $\gamma$ rel 2, in ginbuna crucian carp. FEBS J 281:1046-1056. doi: 10.1111/febs.12666.

47. Gordon S and Taylor PR (2005) Monocyte and macrophage heterogeneity. Nat Rev Immunol 5:953-964. doi: 10.1038/nri1733

48. Gordon S (2007) The macrophage: Past, present and future. Eur J Immunol 37:S9-S17. doi: 10.1002/eji.200737638.

49. Nguyen Chi M, Laplace-Builhe B, Travnickova J, Luz-Crawford P, Tejedor G, Phan QT, DurouxRichard I, Levraud J-P, Kissa K, Lutfalla G, Jorgensen C, and Djouad F (2015) Identification of polarized macrophage subsets in zebrafish. eLife 4:e07288. doi: 10.7554/eLife.07288.

50. Wiens GD and Glenney GW (2011) Origin and evolution of TNF and TNF receptor superfamilies. Dev Comp Immunol 35:1324-1335. doi: 10.1016/j.dci.2011.03.031.

51. Zou J, Peddie S, Scapigliati G, Zhang Y, Bols NC, Ellis AE, and Secombes CJ (2003) Functional characterisation of the recombinant tumor necrosis factors in rainbow trout, Oncorhynchus mykiss. Dev Comp Immunol 27:813-822. doi: 10.1016/S0145-305X(03)00077-6.

52. Grayfer L, Walsh JG, and Belosevic M (2008) Characterization and functional analysis of goldfish (Carassius auratus L.) tumor necrosis factor-alpha. Dev Comp Immunol 32:532-543. doi: 10.1016/j.dci.2007.09.009.

53. Roca FJ, Mulero I, López-Muñoz A, Sepulcre MP, Renshaw SA, Meseguer J, and Mulero V (2008) Evolution of the Inflammatory Response in Vertebrates: Fish TNF- $\alpha$ Is a Powerful Activator of Endothelial Cells but Hardly Activates Phagocytes. J Immunol 181:5071-5081. doi: 10.4049/ jimmunol.181.7.5071.

54. Forlenza M, Magez S, Scharsack JP, Westphal A, Savelkoul HFJ, and Wiegertjes GF (2009) Receptor-Mediated and Lectin-Like Activities of Carp (Cyprinus carpio) TNF- $\alpha$. J Immunol 183:5319-5332. doi: 10.4049/jimmunol.0901780.

55. Hong S, Li R, Xu Q, Secombes CJ, and Wang T (2013) Two Types of TNF- $\alpha$ Exist in Teleost Fish: Phylogeny, Expression, and Bioactivity Analysis of Type-II TNF- $\alpha 3$ in Rainbow Trout Oncorhynchus mykiss. J Immunol 191:5959-5972. doi: 10.4049/jimmunol.1301584.

56. Huising MO, Kruiswijk CP, and Flik G (2006) Phylogeny and evolution of class-I helical cytokines. J Endocrinol 189:1-25. doi: 10.1677/joe.1.06591. 
57. Liongue C and Ward A (2007) Evolution of Class I cytokine receptors. BMC Evolutionary Biology 7:120. doi: 10.1186/1471-2148-7-120.

58. Dijkstra JM (2014) TH2 and Treg candidate genes in elephant shark. 511:E7-E9. doi: 0.1038/ nature13446.

59. Van Dyken SJ and Locksley RM (2013) Interleukin-4- and Interleukin-13-Mediated Alternatively Activated Macrophages: Roles in Homeostasis and Disease. Annu Rev Immunol 31:317-343. doi: 10.1146/annurev-immunol-032712-095906.

60. Mueller TD, Zhang J-L, Sebald W, and Duschl A (2002) Structure, binding, and antagonists in the IL-4/IL-13 receptor system. BBA - Mol Cel Res 1592:237-250. doi: 10.1016/S01674889(02)00318-X.

61. Wang T and Secombes CJ (2015) The evolution of IL-4 and IL-13 and their receptor subunits. Cytokine 75:8-13. doi: 10.1016/j.cyto.2015.04.012.

62. Li J-H, Shao J-Z, Xiang L-X, and Wen Y (2007) Cloning, characterization and expression analysis of pufferfish interleukin-4 cDNA: The first evidence of Th2-type cytokine in fish. Mol Immunol 44:2078-2086. doi: 10.1016/j.molimm.2006.09.010.

63. Ohtani M, Hayashi N, Hashimoto K, Nakanishi T, and Dijkstra J (2008) Comprehensive clarification of two paralogous interleukin 4/13 loci in teleost fish. Immunogenetics 60:383-397. doi: 10.1007/s00251-008-0299-x.

64. Lin A-F, Xiang L-X, Wang Q-L, Dong W-R, Gong Y-F, and Shao J-Z (2009) The DC-SIGN of Zebrafish: Insights into the Existence of a CD209 Homologue in a Lower Vertebrate and Its Involvement in Adaptive Immunity. J Immunol 183:7398-7410. doi: 10.4049/jimmunol.0803955.

65. Hu Y-L, Xiang L-X, and Shao J-Z (2010) Identification and characterization of a novel immunoglobulin $\mathrm{Z}$ isotype in zebrafish: Implications for a distinct B cell receptor in lower vertebrates. Mol Immunol 47:738-746. doi: 10.1016/j.molimm.2009.10.010.

66. Zhu L-y, Pan P-p, Fang W, Shao J-z, and Xiang L-x (2012) Essential Role of IL-4 and IL-4R $\alpha$ Interaction in Adaptive Immunity of Zebrafish: Insight into the Origin of Th2-like Regulatory Mechanism in Ancient Vertebrates. J Immunol 188:5571-5584. doi: 10.4049/jimmunol.1102259.

67. Yamaguchi T, Katakura F, Someya K, Dijkstra JM, Moritomo T, and Nakanishi T (2013) Clonal growth of carp (Cyprinus carpio) T cells in vitro: long-term proliferation of Th2-like cells. Fish Shellfish Immunol 34:433-42. doi: 10.1016/j.fsi.2012.11.005.

68. Chang C-I, Zoghi B, Liao JC, and Kuo L (2000) The Involvement of Tyrosine Kinases, Cyclic AMP/Protein Kinase A, and p38 Mitogen-Activated Protein Kinase in IL-13-Mediated Arginase I Induction in Macrophages: Its Implications in IL-13-Inhibited Nitric Oxide Production. J Immunol 165:2134-2141. doi: 10.4049/jimmunol.165.4.2134.

69. Bystrom J, Evans I, Newson J, Stables M, Toor I, van Rooijen N, Crawford M, ColvilleNash P, Farrow S, and Gilroy DW (2008) Resolution-phase macrophages possess a unique inflammatory phenotype that is controlled by cAMP. Blood 112:4117-4127. doi: 10.1182/ blood-2007-12-129767.

70. Sciaraffia E, Riccomi A, Lindstedt R, Gesa V, Cirelli E, Patrizio M, De Magistris MT, and Vendetti S (2014) Human monocytes respond to extracellular cAMP through A2A and A2B adenosine receptors. J Leukoc Biol 96:113-122. doi: 10.1189/jlb.3A0513-302RR. 
71. Kreider T, Anthony RM, Urban JF, Jr., and Gause WC (2007) Alternatively activated macrophages in helminth infections. Curr Opin Immunol 19:448-53. doi: 10.1016/j.coi.2007.07.002.

72. Raes G, Beschin A, Ghassabeh GH, and De Baetselier P (2007) Alternatively activated macrophages in protozoan infections. Curr Opin Immunol 19:454-459. doi: 10.1016/j. coi.2007.05.007.

73. Sica A and Mantovani A (2012) Macrophage plasticity and polarization: in vivo veritas. J Clin Invest 122:787-795. doi: 10.1172/jci59643.

74. Fast MD, Tse B, Boyd JM, and Johnson SC (2009) Mutations in the Aeromonas salmonicida subsp. salmonicida type III secretion system affect Atlantic salmon leucocyte activation and downstream immune responses. Fish Shellfish Immunol 27:721-728. doi: 10.1016/j. fsi.2009.09.009.

75. Skugor S, Glover KA, Nilsen F, and Krasnov A (2008) Local and systemic gene expression responses of Atlantic salmon (Salmo salar L.) to infection with the salmon louse (Lepeophtheirus salmonis). BMC genomics 9:498. doi: 10.1186/1471-2164-9-498.

76. Saeij JPJ, Stet RJM, Groeneveld A, Verburg-van Kemenade LBM, van Muiswinkel WB, and Wiegertjes GF (2000) Molecular and functional characterization of a fish inducible-type nitric oxide synthase. Immunogenetics 51:339-346. doi: 10.1007/s002510050628.

77. Saeij JP, Van Muiswinkel WB, Groeneveld A, and Wiegertjes GF (2002) Immune modulation by fish kinetoplastid parasites: a role for nitric oxide. Parasitology 124:77-86. doi: 10.1017/ s0031182001008915

78. Joerink M, Forlenza M, Ribeiro CM, de Vries BJ, Savelkoul HF, and Wiegertjes GF (2006) Differential macrophage polarisation during parasitic infections in common carp (Cyprinus carpio L.). Fish Shellfish Immunol 21:561-71. doi: 10.1016/j.fsi.2006.03.006.

79. Forlenza M, Scharsack JP, Kachamakova NM, Taverne-Thiele AJ, Rombout JH, and Wiegertjes GF (2008) Differential contribution of neutrophilic granulocytes and macrophages to nitrosative stress in a host-parasite animal model. Mol Immunol 45:3178-89. doi: 10.1016/j. molimm.2008.02.025.

80. Stolte EH, Savelkoul HF, Wiegertjes G, Flik G, and Lidy Verburg-van Kemenade BM (2008) Differential expression of two interferon-gamma genes in common carp (Cyprinus carpio L.). Dev Comp Immunol 32:1467-81. doi: 10.1016/j.dci.2008.06.012.

81. Forlenza M, Nakao M, Wibowo I, Joerink M, Arts JA, Savelkoul HF, and Wiegertjes GF (2009) Nitric oxide hinders antibody clearance from the surface of Trypanoplasma borreli and increases susceptibility to complement-mediated lysis. Mol Immunol 46:3188-97. doi: 10.1016/j. molimm.2009.08.011.

82. Ribeiro CM, Pontes MJ, Bird S, Chadzinska M, Scheer M, Verburg-van Kemenade BM, Savelkoul HF, and Wiegertjes GF (2010) Trypanosomiasis-induced Th17-like immune responses in carp. PloS one 5:e13012. doi: 10.1371/journal.pone.0013012.

83. Joerink M, Groeneveld A, Ducro B, Savelkoul HF, and Wiegertjes GF (2007) Mixed infection with Trypanoplasma borreli and Trypanosoma carassii induces protection: involvement of crossreactive antibodies. Dev Comp Immunol 31:903-15. doi: 10.1016/j.dci.2006.12.003. 
84. Barreda DR, Hanington PC, and Belosevic M (2004) Regulation of myeloid development and function by colony stimulating factors. Dev Comp Immunol 28:509-554. doi: 10.1016/j. dci.2003.09.010.

85. Wang T, Hanington PC, Belosevic M, and Secombes CJ (2008) Two Macrophage ColonyStimulating Factor Genes Exist in Fish That Differ in Gene Organization and Are Differentially Expressed. J Immunol 181:3310-3322. doi: 10.4049/jimmunol.181.5.3310.

86. Katzenback BA and Belosevic M (2012) Colony-stimulating factor-1 receptor protein expression is a specific marker for goldfish (Carassius auratus L.) macrophage progenitors and their differentiated cell types. Fish Shellfish Immunol 32:434-445. doi: 10.1016/j.fsi.2011.12.003.

87. Barreda DR, Hanington PC, Stafford JL, and Belosevic M (2005) A novel soluble form of the CSF1 receptor inhibits proliferation of self-renewing macrophages of goldfish (Carassius auratus L.). Dev Comp Immunol 29:879-894. doi: 10.1016/j.dci.2005.02.006.

88. Hanington PC, Wang T, Secombes CJ, and Belosevic M (2007) growth factors of lower vertebrates: characterization of goldfish (Carassius auratus L.) Macrophage colony-stimulating factor-1. J Biol Chem 282:31865-31872. doi: 10.1074/jbc.M706278200.

89. Hanington PC, Hitchen SJ, Beamish LA, and Belosevic M (2009) Macrophage colony stimulating factor (CSF-1) is a central growth factor of goldfish macrophages. Fish Shellfish Immunol 26:1-9. doi: 10.1016/j.fsi.2008.09.020.

90. Rieger AM, Hanington PC, Belosevic M, and Barreda DR (2014) Control of CSF-1 induced inflammation in teleost fish by a soluble form of the CSF-1 receptor. Fish Shellish Immunol 41:45-51. doi: 10.1016/j.fsi.2014.03.035.

91. Rieger AM, Havixbeck JJ, Belosevic M, and Barreda DR (2015) Teleost soluble CSF-1R modulates cytokine profiles at an inflammatory site, and inhibits neutrophil chemotaxis, phagocytosis, and bacterial killing. Dev Comp Immunol 49:259-266. doi: 10.1016/j.dci.2014.12.003.

92. Dzik JM (2014) Evolutionary Roots of Arginase Expression and Regulation. Front Immunol 5:544. doi: 10.3389/fimmu.2014.00544.

93. Vodovotz Y (1997) Control of nitric oxide production by transforming growth factor-beta1: mechanistic insights and potential relevance to human disease. Nitric Oxide - Biol Ch 1:3-17. doi: 10.1006/niox.1996.0105.

94. Gitelman SE and Derynck R, Transforming growth factor $\beta$ (TGF- $\beta$ ), in Guidebook to Cytokines and Their Receptors, N. Nicola, Editor. 1994, Oxford University Press: New York. p. 223-226.

95. Wei H, Yin L, Feng S, Wang X, Yang K, Zhang A, and Zhou H (2015) Dual-parallel inhibition of IL-10 and TGF-beta1 controls LPS-induced inflammatory response via NF-kappaB signaling in grass carp monocytes/macrophages. Fish Shellfish Immunol 44:445-452. doi: 10.1016/j. fsi.2015.03.023.

96. Grayfer L, Hodgkinson JW, Hitchen SJ, and Belosevic M (2011) Characterization and functional analysis of goldfish (Carassius auratus L.) interleukin-10. Mol Immunol 48:563-571. doi: 10.1016/j.molimm.2010.10.013.

97. Piazzon MC, Savelkoul HS, Pietretti D, Wiegertjes GF, and Forlenza M (2015) Carp Il10 Has Anti-Inflammatory Activities on Phagocytes, Promotes Proliferation of Memory T Cells, and Regulates B Cell Differentiation and Antibody Secretion. J. Immunol 194:187-199. doi: 10.4049/ jimmunol.1402093. 
98. Windmueller HG and Spaeth AE (1981) Source and fate of circulating citrulline. M J Physiol Cell Physiol 241:E473-480. doi: 10.1152/ajpendo.1981.241.6.E473.

99. Morris SM, Jr. (2006) Arginine: beyond protein. Am J Clin Nutr 83:508s-512s. doi: 10.1093/ ajcn/83.2.508S.

100. Buentello JA and Gatlin III DM (2000) The dietary arginine requirement of channel catfish (Ictalurus punctatus) is influenced by endogenous synthesis of arginine from glutamic acid. Aquaculture 188:311-321. doi: 10.1016/S0044-8486(00)00344-6.

101. Tan J, Liu S, Guo Y, Applegate TJ, and Eicher SD (2014) Dietary l-arginine supplementation attenuates lipopolysaccharide-induced inflammatory response in broiler chickens. Br J Nutr 111:1394-1404. doi: 10.1017/S0007114513003863.

102. Jiang J, Shi D, Zhou X-Q, Hu Y, Feng L, Liu Y, Jiang W-D, and Zhao Y (2015) In vitro and in vivo protective effect of arginine against lipopolysaccharide induced inflammatory response in the intestine of juvenile Jian carp (Cyprinus carpio var. Jian). Fish Shellfish Immunol 42:457-464. doi: 10.1016/j.fsi.2014.11.030.

103. Closs EI, Boissel JP, Habermeier A, and Rotmann A (2006) Structure and function of cationic amino acid transporters (CATs). J Membr Biol 213:67-77. doi: 10.1007/s00232-006-0875-7.

104. Yang J, Tan Q, Zhu W, Chen C, Liang X, and Pan L (2014) Cloning and molecular characterization of cationic amino acid transporter y+LAT1 in grass carp (Ctenopharyngodon idellus). Fish Physiol Biochem 40:93-104. doi: 10.1007/s10695-013-9827-1.

105. Wu G and Morris SM, Jr. (1998) Arginine metabolism: nitric oxide and beyond. Biochem J 336:117. doi: $10.1042 / \mathrm{bj} 3360001$.

106. Radi R, Peluffo G, Alvarez MN, Naviliat M, and Cayota A (2001) Unraveling peroxynitrite formation in biological systems. Free Radic Biol Med 30:463-88. doi: 10.1016/S08915849(00)00373-7.

107. Elks PM, van der Vaart M, van Hensbergen V, Schutz E, Redd MJ, Murayama E, Spaink HP, and Meijer AH (2014) Mycobacteria counteract a TLR-mediated nitrosative defense mechanism in a zebrafish infection model. PloS one 9:e100928. doi: 10.1371/journal.pone.0100928.

108. Venkatesh B (2003) Evolution and diversity of fish genomes. Curr Opin Genet Dev 13:588-92. doi: 10.1016/j.gde.2003.09.001

109. Berthelot C, Brunet F, Chalopin D, Juanchich A, Bernard M, Noel B, Bento P, Da Silva C, Labadie K, Alberti A, Aury JM, Louis A, Dehais P, Bardou P, Montfort J, Klopp C, Cabau C, Gaspin C, Thorgaard GH, Boussaha M, Quillet E, Guyomard R, Galiana D, Bobe J, Volff JN, Genet C, Wincker P, Jaillon O, Roest Crollius H, and Guiguen Y (2014) The rainbow trout genome provides novel insights into evolution after whole-genome duplication in vertebrates. Nat Commun 5:3657. doi: $10.1038 /$ ncomms4657.

110. Henkel CV, Dirks RP, Jansen HJ, Forlenza M, Wiegertjes GF, Howe K, van den Thillart GE, and Spaink HP (2012) Comparison of the exomes of common carp (Cyprinus carpio) and zebrafish (Danio rerio). Zebrafish 9:59-67. doi: 10.1089/zeb.2012.0773.

111. Wang JT, Li JT, Zhang XF, and Sun XW (2012) Transcriptome analysis reveals the time of the fourth round of genome duplication in common carp (Cyprinus carpio). BMC genomics 13:96. doi: 10.1186/1471-2164-13-96. 
112. Blomme T, Vandepoele K, De Bodt S, Simillion C, Maere S, and Van de Peer Y (2006) The gain and loss of genes during 600 million years of vertebrate evolution. Genome biol 7:R43. doi: 10.1186/gb-2006-7-5-r43.

113. Lepiller S, Franche N, Solary E, Chluba J, and Laurens V (2009) Comparative analysis of zebrafish nos2a and nos2b genes. Gene 445:58-65. doi: 10.1016/j.gene.2009.05.016.

114. Hall CJ, Flores MV, Oehlers SH, Sanderson LE, Lam EY, Crosier KE, and Crosier PS (2012) Infection-responsive expansion of the hematopoietic stem and progenitor cell compartment in zebrafish is dependent upon inducible nitric oxide. Cell stem cell 10:198-209. doi: 10.1016/j. stem.2012.01.007.

115. Elks PM, Brizee S, van der Vaart M, Walmsley SR, van Eeden FJ, Renshaw SA, and Meijer AH (2013) Hypoxia inducible factor signaling modulates susceptibility to mycobacterial infection via a nitric oxide dependent mechanism. Genome PLoS pathog 9:e1003789. doi: 10.1371/journal. ppat.1003789.

116. Hegedus Z, Zakrzewska A, Agoston VC, Ordas A, Racz P, Mink M, Spaink HP, and Meijer AH (2009) Deep sequencing of the zebrafish transcriptome response to mycobacterium infection. Mol Immunol 46:2918-30. doi: 10.1016/j.molimm.2009.07.002.

117. Poon KL, Richardson M, and Korzh V (2008) Expression of zebrafish nos2b surrounds oral cavity. Dev Dyn 237:1662-7. doi: 10.1002/dvdy.21566.

118. Wang C, Delcros JG, Cannon L, Konate F, Carias H, Biggerstaff J, Gardner RA, and Phanstiel IVOt (2003) Defining the molecular requirements for the selective delivery of polyamine conjugates into cells containing active polyamine transporters. J Med Chem 46:5129-38. doi: 10.1021/ jm030223a.

119. Jenkinson CP, Grody WW, and Cederbaum SD (1996) Comparative properties of arginases. Comp Biochem Physiol B Biochem Mol Biol 114:107-32. doi: 10.1016/0305-0491(95)02138-8.

120. Crespo CL, Vernieri C, Keller PJ, Garre M, Bender JR, Wittbrodt J, and Pardi R. (2014) The PAR complex controls the spatiotemporal dynamics of F-actin and the MTOC in directionally migrating leukocytes. J Cell Sci Pt 127:4381-95. doi: 10.1242/jcs.146217.

121. Renshaw SA and Trede NS (2012) A model 450 million years in the making: zebrafish and vertebrate immunity. Dis Model Mech 5:38-47. doi: 10.1242/dmm.007138.

122. Henry KM, Loynes CA, Whyte MK, and Renshaw SA (2013) Zebrafish as a model for the study of neutrophil biology. J Leukoc Biol 94:633-42. doi: 10.1189/jlb.1112594.

123. Torraca V, Masud S, Spaink HP, and Meijer AH (2014) Macrophage-pathogen interactions in infectious diseases: new therapeutic insights from the zebrafish host model. Dis Model Mech 7:785-97. doi: 10.1242/dmm.015594.

124. Ellett F, Pase L, Hayman JW, Andrianopoulos A, and Lieschke GJ (2011) mpeg1 promoter transgenes direct macrophage-lineage expression in zebrafish. Blood 117:e49-56. doi: 10.1182/ blood-2010-10-314120.

125. Gray C, Loynes CA, Whyte MK, Crossman DC, Renshaw SA, and Chico TJ (2011) Simultaneous intravital imaging of macrophage and neutrophil behaviour during inflammation using a novel transgenic zebrafish. J Thromb Haemost 105:811-9. doi: 10.1160/th10-08-0525. 


\section{Chapter 2}

126. Renshaw SA, Loynes CA, Trushell DM, Elworthy S, Ingham PW, and Whyte MK (2006) A transgenic zebrafish model of neutrophilic inflammation. Blood 108:3976-8. doi: 10.1182/ blood-2006-05-024075.

127. Mathias JR, Perrin BJ, Liu TX, Kanki J, Look AT, and Huttenlocher A. (2006) Resolution of inflammation by retrograde chemotaxis of neutrophils in transgenic zebrafish. J Leukoc Biol 80:1281-8. doi: 10.1189/jlb.0506346.

128. Cambier CJ, Takaki KK, Larson RP, Hernandez RE, Tobin DM, Urdahl KB, Cosma CL, and Ramakrishnan L (2014) Mycobacteria manipulate macrophage recruitment through coordinated use of membrane lipids. Nature 505:218-22. doi: 10.1038/nature12799.

129. Herbomel P, Thisse B, and Thisse C (1999) Ontogeny and behaviour of early macrophages in the zebrafish embryo. Development 126:3735-45. 
The 'macrophages first' point of view 



\section{Transcriptome sequencing supports a striking conservation of macrophage polarization in fish}

Annelieke S. Wentzel ${ }^{1}$, Jules Petit ${ }^{2}$, Wouter G. van Veen ${ }^{3}$, Inge R. Fink ${ }^{1}$,

Marleen H. Scheer ${ }^{1}$, M. Carla Piazzon ${ }^{4}$, Maria Forlenza ${ }^{1}$, Herman P.

Spaink $^{5}$, Geert F. Wiegertjes ${ }^{2}$

${ }^{1}$ Cell Biology and Immunology Group, ${ }^{2}$ Aquaculture and Fisheries Group, ${ }^{3}$ Experimental

Zoology Group, Wageningen University \& Research Wageningen, Netherlands

4Fish Pathology Group, Institute of Aquaculture Torre de la Sal (IATS-CSIC), Castellón,

Spain.

${ }^{5}$ Institute of Biology, Leiden University, Leiden, Netherlands

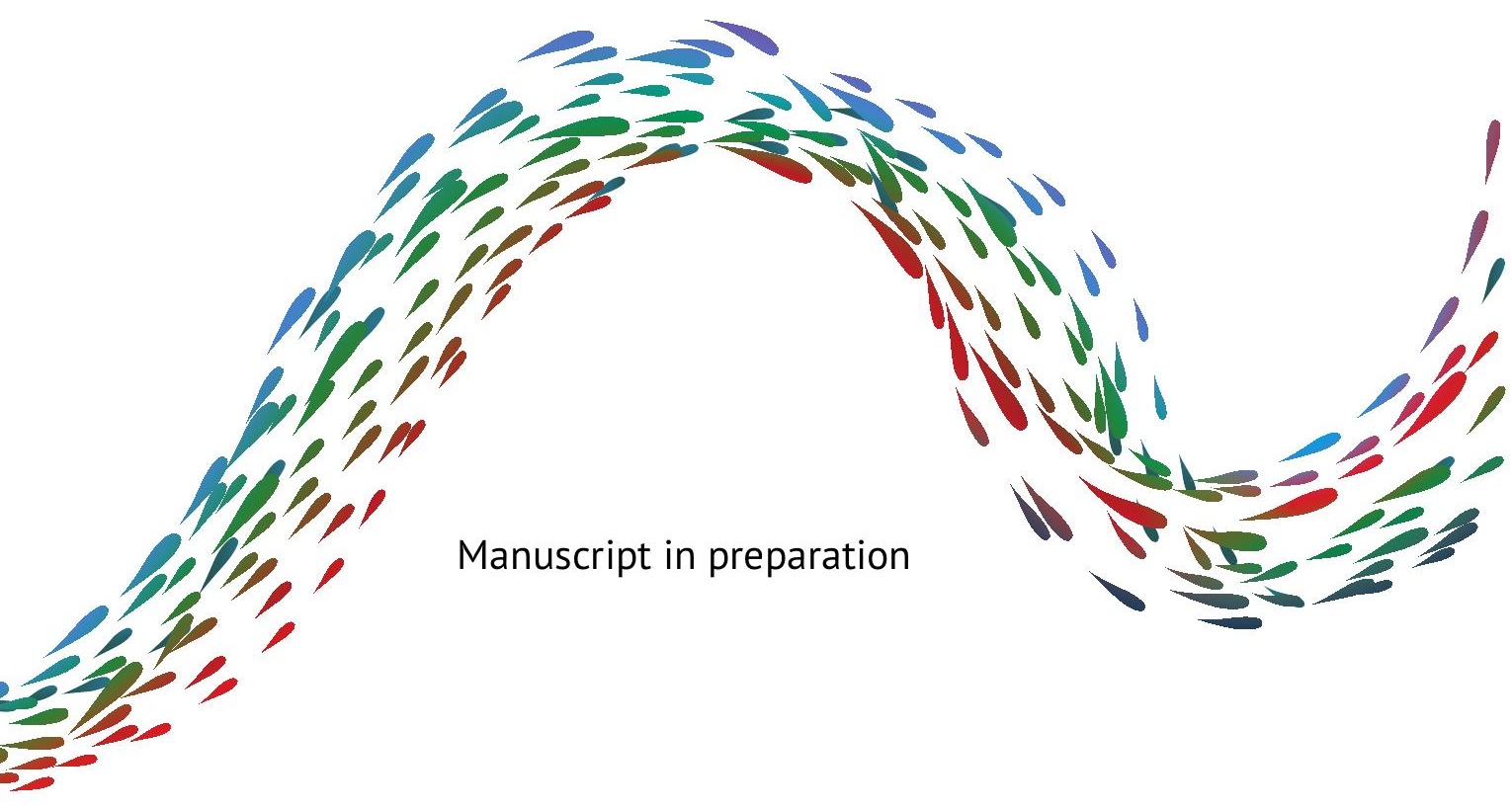




\begin{abstract}
Mammalian macrophages can adopt polarization states that, depending on the exact stimuli present in their extracellular environment, can lead to very different functions. Although these different polarization states have been shown primarily for macrophages of human and mice, it is likely that polarized macrophages with corresponding phenotypes exist across mammals. Evidence of functional conservation in macrophages from teleost fish suggests that the same, or at least comparable polarization states should also be present in teleosts. However, corresponding transcriptional profiles of marker genes have not been reported thus far. In this study we confirm that macrophages from common carp can polarize into M1- and M2 phenotypes with conserved functions and corresponding transcriptional profiles compared to mammalian macrophages. Carp M1 macrophages show increased production of nitric oxide and a transcriptional profile with increased pro-inflammatory cytokines and mediators, including il6, il12 and saa. Carp M2 macrophages show increased arginase activity and a transcriptional profile with increased anti-inflammatory mediators, including cyr61, timp $2 b$ and $\operatorname{tgm} 2 b$. Our RNA sequencing approach allowed us to list, in an unbiased manner, markers discriminating between M1 and M2 macrophages of teleost fish. We discuss the importance of our findings for the evaluation of immunostimulants for aquaculture and for the identification of gene targets to generate transgenic zebrafish for detailed studies on M1 and M2 macrophages. Above all, we discuss the striking degree of evolutionary conservation of macrophage polarization in a lower vertebrate.
\end{abstract}




\section{Introduction}

Depending on stimuli present in their extracellular environment, mammalian macrophages can adopt polarization states that can exert very different, sometimes opposite, functions. These opposite functional differences were initially referred to as the M1/M2 paradigm [1], in which M1 macrophages exert pro-inflammatory activities driven by Th1 cytokines as opposed to M2 macrophages that would be driven by Th2 cytokines and be involved in anti-inflammatory responses. This paradigm is primarily based on arginine metabolism, as inflammatory M1 macrophages metabolize arginine to produce anti-microbial nitric oxide (NO) while anti-inflammatory M2 macrophages utilize the same arginine to produce proline and polyamines required for cell proliferation and tissue generation. In more recent studies, the M1/M2 paradigm has been refined to include at least nine distinct macrophage activation states [2] or define M1 and M2 macrophages at the opposite ends of an entire spectrum of activation states [3, 4]. Different macrophage polarization states have been studied in detail in mice and men, however it remains unclear to what extend these polarized phenotypes are conserved in nonmammalian species. Although considerable differences exist between polarized macrophages of mammals including mice and men [5-7], their M1 and M2 macrophages display comparable core phenotypes and it is likely that polarized macrophages with corresponding core phenotypes exist throughout mammals. Based on our previous work [8,9] we hypothesize that these comparable core phenotypes would also be displayed by macrophages of common carp (Cyprinus carpio), a teleost species that shared the last tetrapod's common ancestor more than 350 million years ago and is an important species for aquaculture [10].

In fish, the ability of macrophages to polarize towards M1-like and M2-like states has been demonstrated [11-13]. In carp, we previously showed that macrophages assume an inflammatory phenotype in response to lipopolysaccharide (LPS) stimulation [8] in the presence or absence of interferon-gamma (Ifn- $\gamma$ ) [14]. This phenotype is characterized by the production of NO (mice) and pro-inflammatory cytokines similar to mammalian M1 macrophages when stimulated with LPS alone or in combination with IFN- $\gamma$, or granulocyte macrophage-colony stimulating factor (GM-CSF) [15]. At the other end of the spectrum, cyprinid macrophages adopt an anti-inflammatory phenotype characterized by elevated arginase activity when stimulated with Il-4/13 $[16,17]$ or cAMP $[8,16]$. This phenotype is similar to mammalian M2 macrophages polarized by macrophage-colony stimulating 
factor (M-CSF), interleukin-4 (IL-4), interleukin 13 (IL-13) or extracellular cAMP [18], which show comparable increased production of anti-inflammatory cytokines [15]. In vivo, macrophage polarization has been studied using tnf $\alpha /$ mpeg1 [19] double transgenic zebrafish (Danio rerio) and preliminary findings show differences in expression of pro- and anti-inflammatory markers in $t n f \alpha^{+}$and $t n f \alpha^{-}$ macrophages. In addition, some work towards a full transcriptional phenotype has been undertaken in tnf $\alpha /$ mpeg1 double transgenic zebrafish [20] and other teleosts $[21,22]$. Taken together, these studies provide the foundation to understand macrophage polarization in fish. However, a comprehensive transcriptomic analysis using known modulators of M1 and M2 polarization, associated with phenotypic validation through robust functional assays, is still lacking.

In this study, we used our well-established in vitro carp macrophage model and combined the phenotypically validated M1 and M2 macrophages with an unbiased transcriptome analysis to elucidate the transcriptional profile of M1 and M2 macrophages in a lower vertebrate. As such, we started with the functionally opposite ends of the macrophage spectrum, M1 and M2 extremes, which serve as a stable framework to determine evolutionary conserved polarization profiles. This allows us to comparatively study macrophage polarization across vertebrates and to identify a comprehensive set of genes that can be used as potential markers across species. In doing so, we provide insight into the conservation of macrophage polarization beyond mammals.

\section{Results}

\section{Polarized macrophages show differences in morphology and in function}

We studied the phenotype of stimulated carp macrophages to confirm their polarization state prior to transcriptome analysis. When macrophages were stimulated with LPS or cAMP, to obtain M1 or M2 polarization states respectively, we observed a change in morphology. During the polarization period of 24 hours, when compared to unstimulated cells from the same individual, M1 macrophages adhered to the culture surface and assumed flattened, irregular shapes with multiple membrane protrusions, while M2 macrophages retained a rounded shape with only few protrusions (Figure 3.1A). In addition, M1 macrophages formed a higher number of large, multinuclear cells (sometimes referred to as giant cells) compared to M2 macrophages or unstimulated controls. This difference in morphology was 
mirrored by clear differences in functional phenotypes. Analysis of two canonical macrophage functions, NO production (Figure 3.1B) and arginase activity (Figure 3.1C), showed clear differences between M1 and M2 macrophages. M1 macrophages showed a significantly higher NO production than M2 and unstimulated controls, reflecting a pro-inflammatory phenotype. In contrast, M2 macrophages did not produce any NO and showed a significantly higher intracellular arginase activity than M1 and unstimulated macrophages. Analysis of phagocytic activity or capacity, another canonical function of macrophages which is not clearly linked to polarization, did not show clear differences between M1 and M2 macrophages (Figure 3.1D and 3.1E). The clear functional difference in NO production and arginase activity indicates the capacity of teleost macrophages to assume M1 and M2 phenotypes similar to those in mammals.

\section{M1 and M2 carp macrophages display distinct gene expression profiles}

After observing clear morphological and functional differences between M1 and M2 carp macrophages, we examined their transcriptome to explore the differences in expression profiles. DESeq2 analysis resulted in 3396 significantly regulated genes in M1 macrophages and 6142 significantly regulated genes in M2 macrophages, compared to unstimulated control macrophages. Of those significantly regulated genes, expression of 1479 (M1) and 2494 (M2) genes was at least 2-fold increased or 2 -fold decreased (log2 fold change $>1$ or $\log 2$ fold change $<-1$ ) and was thus defined as differentially expressed. Comparison of these genes (Figure 3.2A) showed a clearly distinct expression profile since, besides the 546 genes regulated in both M1 and M2 macrophages, the majority was regulated only in M1 (63\%, 933 genes) or only in M2 (72\%, 1948 genes). Overall, more genes were up- than downregulated, over $70 \%$ of which was upregulated either only in M1- or only in M2 macrophages, while 308 genes were upregulated in either group, representing less than 30\% overlap (Figure 3.2B). Similarly, at least $64 \%$ of downregulated genes are specific to either M1 or M2 macrophages, while only $36 \%$ or less overlapped (Figure 3.2C). Taken together, these results show two distinct transcriptional profiles for polarized M1 and M2 carp macrophages. 
A
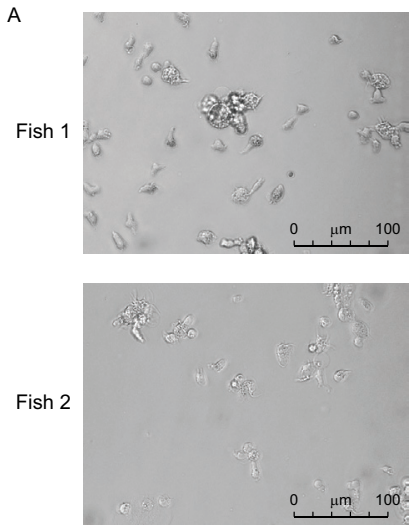

C

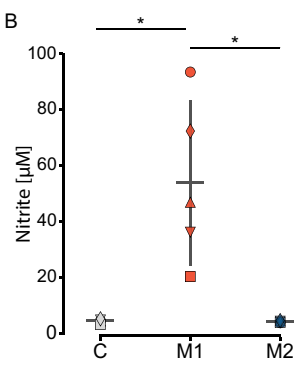

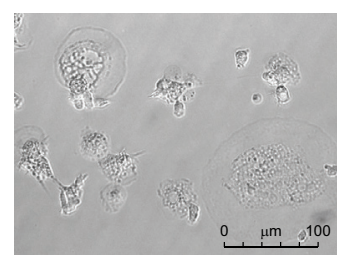

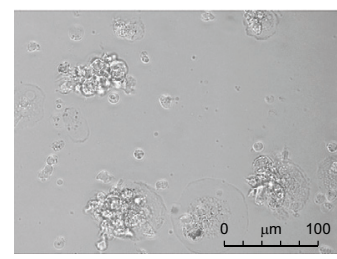

M1
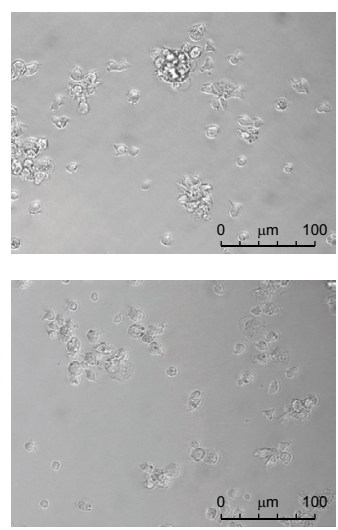

M2
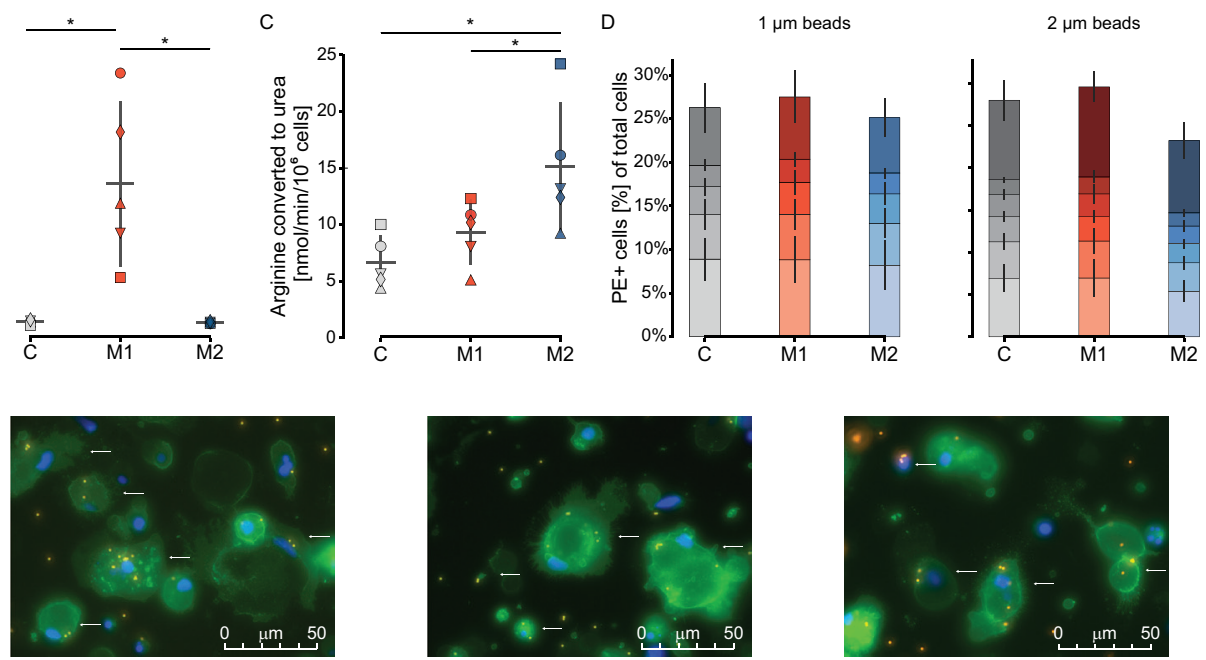

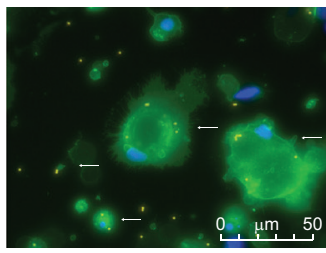

M1

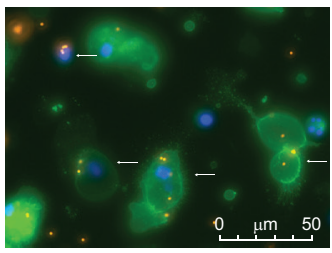

M2

Figure 3.1 Polarized carp macrophages display different functional phenotypes. Phenotypical differences in carp macrophages either polarized for $24 \mathrm{~h}$ with $30 \mu \mathrm{g} / \mathrm{ml}$ LPS (M1) or $0.5 \mu \mathrm{g} / \mathrm{ml}$ cAMP (M2), or kept as unstimulated control (C) macrophages. A) Representative images of macrophages from two individual carp, showing morphological differences. B) Nitric oxide production of control (grey), M1 (red) and M2 (blue) treated macrophages measured as nitrite concentration in culture supernatants after $24 \mathrm{~h}$. Symbols indicate individual fish. C) Arginase activity of control (grey), M1 (red) and M2 (blue) stimulated macrophages measured in cell lysates as conversion of L-arginine to urea by arginase in nmol/ $\mathrm{min} / 10^{6}$ cells. Symbols indicate individual fish. D) Phagocytosis of 1 or 2 um fluorescent polystyrene beads, measured by flow cytometry in macrophages stimulated for $24 \mathrm{~h}$ and subsequently incubated for $2 \mathrm{~h}$ with beads in a 10/1 bead/cell ratio. Increasing color intensity represents an increasing number of internalized beads. E) Fluorescent images showing phagocytosis by macrophages stimulated for $24 \mathrm{~h}$ and subsequently incubated for $2 \mathrm{~h}$ with beads in a 10/1 bead/cell ratio. Images show WGA surface staining in green, nuclei in blue and $1 \mu \mathrm{m}$ florescent polystyrene beads in orange after $2 \mathrm{~h}$ incubation with beads. The combination of harvest after stimulation and the presence of beads in the well influences morphologies to become different from those in panel A. Arrows indicate examples of cells that have 
internalized beads. Data are the mean and standard deviation of $n=5$ individual fish (B-D). Data were analyzed using a repeated measures ANOVA with Sidak post-hoc tests for NO and arginase assays (B, C). Data were analyzed using a repeated measures ANOVA to determine differences between control, M1 and M2 groups, either for total or a specific number of beads phagocytosed (D). Differences were considered significant when $p<0.05\left(^{*}\right)$. In cases where sphericity was violated, the Geisser-Greenhouse correction was applied.

Distinct transcriptional profiles feature conserved pro- and anti-inflammatory genes.

To further define the expression profiles of M1 and M2 polarized macrophages, we focused the analysis to those genes that are not only differentially but also substantially expressed in either polarized or unstimulated control macrophages. We define genes substantially expressed when RPKM > 50 in either polarized or unstimulated control macrophages and for upregulated genes a log2 fold change $>1$ ). In our dataset, these represent approximately $7-10 \%$ of all significantly regulated genes. Here we only highlighted the genes that show the highest (top 20) fold change in expression in M1 (Table 3.1) or M2 (Table 3.2) macrophages compared to unstimulated controls. Only four out of the 20 most upregulated genes show overlap between M1 and M2
A

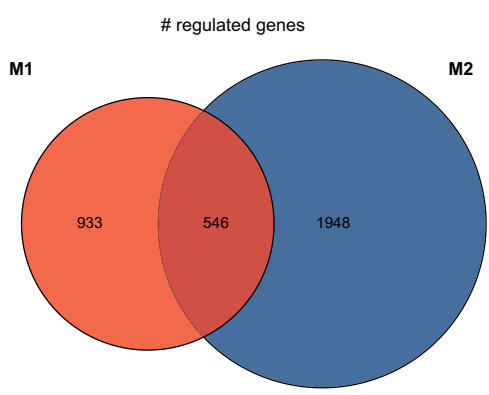

B \# upregulated genes

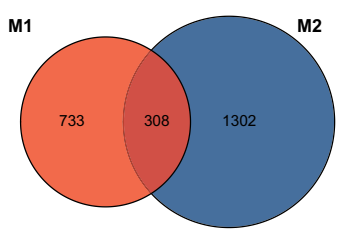

C \# downregulated genes

M1

M2

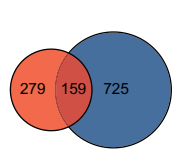

Figure 3.2 M1 and M2 carp macrophages show distinct transcriptional profiles. Proportional Venn diagrams depicting transcriptional changes of carp macrophages polarized for $6 \mathrm{~h}$ with $30 \mu \mathrm{g} / \mathrm{ml}$ LPS (M1, red) or $0.5 \mu \mathrm{g} / \mathrm{ml}$ cAMP (M2, blue) compared to unpolarized control macrophages. The total number of significantly $\left(p_{\text {adjusted }}<0.05\right)$ regulated genes $(\mathbf{A})$ is further specified to show the number of upregulated (B) and downregulated (C) genes. Data are of $n=3$ fish.

macrophages. These are genes involved in general cellular (activation) processes such as cytoskeleton formation (agrn), growth-factor signaling (shc2) and aminoacid metabolism $(t d h)$. Although we observed the canonical pro-inflammatory cytokine il1 $\beta$ in both groups, expression was approximately 30 times higher in M1 than in M2 macrophages, which indicates it acts primarily as a pro-inflammatory M1 gene. 
Next to il $1 \beta$, many other genes among the 20 most upregulated genes in M1 macrophages agree with the prototypical M1 profile. This includes proinflammatory cytokines $i l 12 p 35$ and il6, the acute phase protein serum amyloid $a$ (saa) and genes contributing to or protecting from oxidative stress (nos $2 b$, irg1, lacc1 and cygb1). These genes do not only functionally suit an inflammatory profile, but many of these genes have also been previously linked to human or murine M1 polarized macrophages.

Table 3.1 Transcriptional phenotype of carp M1 macrophages shows high increases in inflammatory mediators and M1 markers. Genes most upregulated (top 20) in M1 macrophages polarized with $30 \mu \mathrm{g} / \mathrm{ml}$ LPS for $6 \mathrm{~h}$ in descending order of fold change gene expression. Genes were included only when all of the following criteria were met: $p_{\text {adiusted }}<0.05$ and average reads per kilobasepair per million reads $(\mathrm{RPKM})>50$ in stimulated or control samples. The 20 most highly upregulated distinct genes were depicted with the gene abbreviation (Gene), gene description, gene identifier (Gene ID cypCar), log2 fold change compared to unstimulated control macrophages (Log2FC), short description of their main function (in macrophages if possible) and average RPKM in control (C) and LPS polarized macrophages. Multiple cypCar IDs per gene were included only if RPKM of both paralogs fell within the top 20 most upregulated genes. Each cypCar gene ID represents an individual gene sequence unless combined by a dash (-), indicating a possible mis-annotation of a single gene as two separate genes. Data are of $n=3$ fish.

\begin{tabular}{|c|c|c|c|c|c|c|}
\hline Gene & Gene description & $\begin{array}{l}\text { Gene ID } \\
\text { cypCar }\end{array}$ & $\begin{array}{l}\log 2 \\
\text { FC }\end{array}$ & Main Function & $\begin{array}{l}\text { RPKM } \\
\quad \text { C }\end{array}$ & $\begin{array}{l}\text { RPKM } \\
\text { M1 }\end{array}$ \\
\hline il12p35 & $\begin{array}{l}\text { Interleukin } 12 \\
\text { subunit alpha } \\
\text { (p35) }\end{array}$ & $\begin{array}{l}00024698- \\
00024699\end{array}$ & $\begin{array}{l}8.8 \\
7.2\end{array}$ & $\begin{array}{l}\text { P35 subunit of the pro-inflammatory cytokine II-12. } \\
\text { Involved in the activation of Th1 and NK cells [23]. }\end{array}$ & $\begin{array}{l}0.1 \\
0.4\end{array}$ & $\begin{array}{l}56.0 \\
59.4\end{array}$ \\
\hline il1 $\beta$ & Interleukin 1 beta & $\begin{array}{l}00043439- \\
00043440\end{array}$ & $\begin{array}{l}7.6 \\
7.5\end{array}$ & $\begin{array}{l}\text { Pro-inflammatory cytokine. Mediator of various cellular } \\
\text { activities including proliferation, differentiation and } \\
\text { apoptosis [24]. }\end{array}$ & $\begin{array}{l}72.0 \\
74.0\end{array}$ & $\begin{array}{l}12832.1 \\
11482.7\end{array}$ \\
\hline steap4 & $\begin{array}{l}\text { Six- } \\
\text { transmembrane } \\
\text { epithelial antigen } \\
\text { of prostate } 4\end{array}$ & 00042005 & 7.0 & $\begin{array}{l}\text { Metalloreductase involved in the transfer of ions from } \mathrm{Fe}_{3}^{+} \\
\text {and } \mathrm{Cu}_{2}^{+} \text {to NAD and plays a role in cellular homeostasis } \\
\text { during inflammation. Increased Steap4 may reduce } \\
\text { circulating iron available for parasites [25]. }\end{array}$ & 4.6 & 405.9 \\
\hline agrn & Agrin & 00029572 & 7.0 & $\begin{array}{l}\text { Extracellular-matrix protein involved in monocyte/ } \\
\text { macrophage survival, cytoskeleton formation and } \\
\text { phagocytosis [26]. }\end{array}$ & 32.7 & 2569.8 \\
\hline saa & $\begin{array}{l}\text { Serum amyloid A } \\
\text { protein }\end{array}$ & $\begin{array}{l}00037333 \\
00036204\end{array}$ & $\begin{array}{l}6.3 \\
5.3\end{array}$ & $\begin{array}{l}\text { Acute phase protein, chemotactic to phagocytes and } \\
\text { induces transcription of several pro-inflammatory } \\
\text { cytokines }[27,28] \text {. }\end{array}$ & $\begin{array}{c}2.8 \\
77.6\end{array}$ & $\begin{array}{c}183.8 \\
1968.3\end{array}$ \\
\hline $\begin{array}{l}\text { ptgs } 2 a \text { or } \\
\text { cox2 }\end{array}$ & $\begin{array}{l}\text { Prostaglandin- } \\
\text { endoperoxide } \\
\text { synthase } 2 a\end{array}$ & 00026925 & 5.7 & $\begin{array}{l}\text { Also known as Cox-2. Increased expression in human M1 } \\
\text { macrophages }[29,30] .\end{array}$ & 8.7 & 319.8 \\
\hline olfm4 & $\begin{array}{l}\text { Olfactomedin- } \\
\text { 4-like }\end{array}$ & 00047183 & 5.4 & $\begin{array}{l}\text { Extracellular glycoprotein indicated in myeloid-specific } \\
\text { differentiation and neutrophil inflammation [31-33]. }\end{array}$ & 1.8 & 69.5 \\
\hline lacc1 & $\begin{array}{l}\text { Laccase-domain } \\
\text { containing } \\
\text { protein } 1\end{array}$ & 00009189 & 5.3 & $\begin{array}{l}\text { Promotes fatty-acid oxidation, inflammasome activation, } \\
\text { mitochondrial and NADPH-oxidase-dependent reactive } \\
\text { oxygen species production and bactericidal activity of } \\
\text { macrophages [34]. }\end{array}$ & 4.3 & 137.2 \\
\hline$n o s 2 b$ & $\begin{array}{l}\text { Nitric oxide } \\
\text { synthase } 2 b\end{array}$ & $\begin{array}{l}00004424 \\
00024539\end{array}$ & $\begin{array}{l}5.3 \\
5.2\end{array}$ & $\begin{array}{l}\text { Production of antimicrobial nitric oxide. Has functioned as } \\
\text { M2 marker since macrophage polarization was described } \\
{[1,35] \text {. }}\end{array}$ & $\begin{array}{l}10.1 \\
43.6\end{array}$ & $\begin{array}{c}355.4 \\
1427.6\end{array}$ \\
\hline
\end{tabular}




\begin{tabular}{|c|c|c|c|c|c|c|}
\hline Gene & Gene description & $\begin{array}{l}\text { Gene ID } \\
\text { cypCar }\end{array}$ & $\begin{array}{l}\text { Log2 } \\
\text { FC }\end{array}$ & Main Function & $\begin{array}{l}\text { RPKM } \\
\quad C\end{array}$ & $\begin{array}{c}\text { RPKM } \\
\text { M1 }\end{array}$ \\
\hline mecr & $\begin{array}{l}\text { Mitochondrial } \\
\text { Enoyl-[acyl- } \\
\text { carrier-protein] } \\
\text { reductase }\end{array}$ & $\begin{array}{l}00002503- \\
00002502\end{array}$ & $\begin{array}{l}5.1 \\
5.0\end{array}$ & $\begin{array}{l}\text { Protein involved in mitochondrial atty acid synthesis. } \\
\text { Increased upon Salmonella enteritidis infection in chicken } \\
\text { macrophages [36]. }\end{array}$ & $\begin{array}{l}13.6 \\
24.9\end{array}$ & $\begin{array}{l}340.7 \\
627.8\end{array}$ \\
\hline il6 & Interleukin-6 & 00035927 & 5.0 & $\begin{array}{l}\text { Pro- and anti-inflammatory cytokine produced by } \\
\text { macrophages in response to PRR activation }[15,37] .\end{array}$ & 30.2 & 864.5 \\
\hline$t d h$ & $\begin{array}{l}\text { L-threonine } \\
\text { dehydrogenase }\end{array}$ & 00008269 & 4.6 & $\begin{array}{l}\text { Converts L-theonine into glycine. Glycine modulates } \\
\text { macrophage activity, plays a role in preventing pyroptosis } \\
\text { and shows cytoprotective effects under hypoxia and } \\
\text { oxidant injury }[38,39] \text {. }\end{array}$ & 16.3 & 356.7 \\
\hline $\begin{array}{l}\text { acod1 or } \\
\text { irg1 }\end{array}$ & $\begin{array}{l}\text { Aconitate } \\
\text { decarboxylase } \\
1 / \text { Immune } \\
\text { responsive gene } 1\end{array}$ & $\begin{array}{l}00007903 \\
00026281\end{array}$ & $\begin{array}{l}4.6 \\
4.5\end{array}$ & $\begin{array}{l}\text { Catalyzes production of itaconate. High expression in } \\
\text { mammalian M1 macrophages contributes to metabolic } \\
\text { reprogramming }[40,41] \text {. }\end{array}$ & $\begin{array}{c}71.5 \\
6.4\end{array}$ & $\begin{array}{c}1404.8 \\
121.8\end{array}$ \\
\hline$c y g b 1$ & Cytoglobin 1 & 00046202 & 4.3 & $\begin{array}{l}\text { Oxygen-carrying globin, expressed in macrophages and } \\
\text { increased during oxidative stress. Protection mechanism } \\
\text { against oxidative stress }[42,43] \text {. }\end{array}$ & 3.0 & 56.4 \\
\hline cxcl13 & $\begin{array}{l}\text { C-X-C motif } \\
\text { chemokine ligand } \\
13\end{array}$ & 00002926 & 4.0 & $\begin{array}{l}\text { B-cell chemoattractant. Upregulated in human M1 } \\
\text { macrophages [29]. }\end{array}$ & 8.6 & 96.4 \\
\hline$c x c / 8 / 1$ & $\begin{array}{l}\text { C-X-C motif } \\
\text { chemokine ligand } \\
8 \text { like } 1\end{array}$ & 00016657 & 4.0 & $\begin{array}{l}\text { Previously known as Cxca, Teleost specific Cxcl8-like } \\
\text { cytokine [44]. Recruits neutrophils through CXCR2 [45, } \\
\text { 46]. }\end{array}$ & 230.4 & 2984.1 \\
\hline tymp & $\begin{array}{l}\text { Thymidine } \\
\text { phosphorylase }\end{array}$ & $\begin{array}{l}00038018 \\
00038017\end{array}$ & $\begin{array}{l}3.7 \\
3.6\end{array}$ & $\begin{array}{l}\text { Also known as platelet-derived endothelial-cell growth } \\
\text { factor. Angiogenic factor expressed in macrophages [47]. }\end{array}$ & $\begin{array}{l}24.8 \\
22.4\end{array}$ & $\begin{array}{l}215.9 \\
204.8\end{array}$ \\
\hline $\begin{array}{l}\text { si:ch1073- } \\
\text { 67j19.1 }\end{array}$ & Unknown protein & 00039673 & 3.6 & & 314.3 & 3261.0 \\
\hline tnfrsf11b & $\begin{array}{l}\text { tumor necrosis } \\
\text { factor receptor } \\
\text { superfamily, } \\
\text { member } 11 \mathrm{~b}\end{array}$ & 00045494 & 3.6 & $\begin{array}{l}\text { Also known as osteoprotegerin, a secreted RANKL decoy } \\
\text { receptor. Correlates with inos+ macrophages antiapoptotic } \\
\text { signal in DC leading to increased T-cell activation [48]. }\end{array}$ & 24.6 & 247.3 \\
\hline shc2 & $\begin{array}{l}\text { SHC transforming } \\
\text { protein } 2\end{array}$ & 00020157 & 3.5 & $\begin{array}{l}\text { Mediator of certain growth-factor signaling cascades. } \\
\text { Implicated in cellular proliferation, differentiation, survival } \\
\text { and migration [49]. }\end{array}$ & 4.9 & 50.3 \\
\hline
\end{tabular}

[1] Mills et al., 2000, [15] Mantovani et al., 2004, [23] Wojno et al. 2019, [24] Mantovani et al., 2019, [25] Scarl et al, 2017, [26] Mazzon et al., 2012, [27] Badolato et al., 1994, [28] He et al., 2009, [29] Martinez et al., 2006, [30] Jablonski et al., 2015, [31] Zhang 2002, [32] Clemmensen et al., 2012, [33] Alder et al.,2018, [34] Lahiri et al., 2017, [35] Nathan et al., 1991, [36] Sekelova et al., 2017, [37] Beyer et al., 2012, [38] Carmans et al., 2010, [39] Loomis et al., 2019, [40] Van den Bossche et al., 2017, [41] O’Neill et al., 2019, [42] Li et al., 2007, [43] Oleksiewicz et al., 2011, [44] Abdelkhalek et al., 2009, [45] Deng et al., 2013, [46] de Oliveira et al., 2013, [47] Fox et al., 1995, [48] Walsh and Choi, 2014, [49] Ahmed and Prigent, 2017.

Many of the 20 most upregulated genes in M2 macrophages (Table 3.2) agree with the prototypical M2 profile as described in mammals. Some of these have even been proposed as M2 markers, such as cyr61(11), timp2(b) and tgm2(b). Other genes, such as vegfa(a) and csnrp1(a), have been linked to M2 profiles via transcriptional studies in mammals or can be linked to M2 macrophages on a functional level. For example, some genes are involved in angiogenesis and wound healing (hegf(b), tgm1l, vegfa(a), cyr61(11)), while others facilitate either transcription (crem ( $a$ and $b$ ) or the presence of M2 associated receptors (ramp2). Overall, the transcriptional M1 
Table 3.2 Transcriptional phenotype of carp M2 macrophages shows high increases in mediators of tissue regeneration and M2 markers. Genes most upregulated (top 20) in M2 macrophages polarized with $0.5 \mathrm{mg} / \mathrm{ml}$ cAMP for $6 \mathrm{~h}$ in descending order of fold change gene expression. Genes were included only when all of the following criteria were met: $p_{\text {adiusted }}<0.05$ and average reads per kilobasepair per million reads $(\mathrm{RPKM})>50$ ) in stimulated or control samples. The 20 most highly upregulated genes (excluding paralogs) were depicted with the gene abbreviation (Gene), gene description, gene identifier (Gene ID cypCar), log2 fold change in compared to unstimulated control macrophages (Log2FC), short description of their main function and average RPKM in control (C) and cAMP polarized macrophages. Multiple cypCar IDs per gene were included only if RPKM of both paralogs fell within the top 20 most upregulated genes. Each cypCar gene ID represents an individual gene sequence. unless combined by a dash (-), indicating a possible mis-annotation of a single gene as two separate genes. Data are of $n=3$ fish.

\begin{tabular}{|c|c|c|c|c|c|c|}
\hline Gene & Gene description & $\begin{array}{l}\text { Gene ID } \\
\text { cypCar }\end{array}$ & $\begin{array}{l}\log 2 \\
\text { FC }\end{array}$ & Main Function & $\begin{array}{l}\text { RPKM } \\
\text { C }\end{array}$ & $\begin{array}{l}\text { RPKM } \\
\text { M2 }\end{array}$ \\
\hline cyr61/1 & $\begin{array}{l}\text { Cysteine-rich } \\
\text { angiogenic } \\
\text { inducer } 61 \\
\text { protein-like } \\
\text { protein } 1\end{array}$ & 00001309 & 9.4 & $\begin{array}{l}\text { Also known as cnn1. Extracellular matrix protein involved } \\
\text { in angiogenesis and regulation of matrix remodeling in } \\
\text { cutaneous wound healing. Drives an anti-inflammatory } \\
\text { transcriptional profile }[50,51]\end{array}$ & 0.2 & 103.8 \\
\hline timp $2 b$ & $\begin{array}{l}\text { Tissue inhibitor of } \\
\text { metallo-proteinase } \\
2 \mathrm{~b}\end{array}$ & $\begin{array}{l}00030755 \\
00034223\end{array}$ & $\begin{array}{l}8.0 \\
4.2\end{array}$ & $\begin{array}{l}\text { Inhibits metalloproteinases and is involved in extracellular } \\
\text { matrix remodeling. Decreased in M1 macrophages and } \\
\text { increased in M2 [52]. }\end{array}$ & $\begin{array}{c}31.1 \\
194.3\end{array}$ & $\begin{array}{l}6476.0 \\
2787.4\end{array}$ \\
\hline $\operatorname{tg} m 2 b$ & $\begin{array}{l}\text { Transglutaminase } \\
2 \mathrm{~b} \text { protein }\end{array}$ & $\begin{array}{l}00034483- \\
00030329 \\
00041907\end{array}$ & $\begin{array}{l}7.4 \\
6.9 \\
5.0\end{array}$ & $\begin{array}{l}\text { Ca2+-dependent cross-linking enzyme important in } \\
\text { apoptotic cell clearance by phagocytosis and regulation } \\
\text { of pro-inflammatory cytokine production. Conserved M2 } \\
\text { marker in human and murine M2 macrophages [53-55]. }\end{array}$ & $\begin{array}{l}6.0 \\
6.4 \\
1.7\end{array}$ & $\begin{array}{c}784.6 \\
698.7 \\
68.2\end{array}$ \\
\hline ramp2 & $\begin{array}{l}\text { Receptor activity } \\
\text { modifying } \\
\text { protein } 2\end{array}$ & 00022158 & 6.4 & $\begin{array}{l}\text { Involved in glycosylation and transportation of the } \\
\text { adrenomedullin receptor to the cell surface [56]. } \\
\text { Adrenomedullin is associated with angiogenesis and M2 } \\
\text { macrophage phenotypes, especially in the context of } \\
\text { cancer }[57,58] \text {. }\end{array}$ & 1.8 & 100.9 \\
\hline $\begin{array}{l}\text { dfna5a or } \\
\text { gsdmea }\end{array}$ & $\begin{array}{l}\text { Deafness } \\
\text { autosomal } \\
\text { dominant } 5 \mathrm{a} / \\
\text { Gasdermin Ea }\end{array}$ & 00035581 & 5.1 & $\begin{array}{l}\text { Considered the functional homologue in zebrafish of } \\
\text { human gasdermin E. Although generally an effector } \\
\text { of pyroptosis, that role has been recently questioned } \\
\text { specifically in macrophages [59-61] }\end{array}$ & 12.8 & 421.8 \\
\hline $\arg 2$ & Arginase 2 & 00034978 & 4.8 & $\begin{array}{l}\text { Arginase } 1 \text { is the canonical M2 marker in murine M2 } \\
\text { macrophages. In human M2 macrophages dependent on } \\
\text { the study }[1,62] \text {. }\end{array}$ & 19.9 & 445.2 \\
\hline agrn & Agrin & 00029572 & 4.3 & $\begin{array}{l}\text { Extracellular-matrix protein involved in monocyte/ } \\
\text { macrophage survival, cytoskeleton formation and } \\
\text { phagocytosis [26]. }\end{array}$ & 11.2 & 153.8 \\
\hline$p d e 4 b b$ & $\begin{array}{l}\text { Phospho- } \\
\text { diësterase } 4 b\end{array}$ & $\begin{array}{l}00024882 \\
00020192\end{array}$ & $\begin{array}{l}4.3 \\
3.6\end{array}$ & $\begin{array}{l}\text { Degrades second messenger cAMP, promoting pro- and } \\
\text { regulating anti-inflammatory effects [63-65]. }\end{array}$ & $\begin{array}{l}17.4 \\
25.4\end{array}$ & $\begin{array}{l}284.8 \\
266.8\end{array}$ \\
\hline vegfaa & $\begin{array}{l}\text { Vascular } \\
\text { endothelial growth } \\
\text { factor } \mathrm{Aa}\end{array}$ & 00013154 & 4.3 & $\begin{array}{l}\text { Signaling protein involved in angiogenesis and tissue } \\
\text { generation. Upregulated in M2 macrophages [66, 67]. }\end{array}$ & 4.9 & 77.9 \\
\hline csrnp1a & $\begin{array}{l}\text { Cysteine-serine- } \\
\text { rich nuclear } \\
\text { protein 1a }\end{array}$ & 00015701 & 4.2 & $\begin{array}{l}\text { Transcriptional activator involved in Wnt-signaling and } \\
\text { involved in primitive hematopoiesis in zebrafish[68]. } \\
\text { Upregulated in macrophages of different origins with } \\
\text { multiple stimuli including murine BMDM with II-13 [69] or } \\
\text { LPS [70]. }\end{array}$ & 7.5 & 117.1 \\
\hline il1 $\beta$ & Interleukin 1 beta & $\begin{array}{l}00043439 \\
00043440\end{array}$ & $\begin{array}{l}4.2 \\
4.0\end{array}$ & $\begin{array}{l}\text { Pro-inflammatory cytokine. Mediator of various cellular } \\
\text { activities including proliferation, differentiation and } \\
\text { apoptosis [24]. }\end{array}$ & $\begin{array}{l}46.1 \\
42.2\end{array}$ & $\begin{array}{l}945.4 \\
771.8\end{array}$ \\
\hline
\end{tabular}




\begin{tabular}{|c|c|c|c|c|c|c|}
\hline Gene & Gene description & $\begin{array}{l}\text { Gene ID } \\
\text { cypCar }\end{array}$ & $\begin{array}{l}\log 2 \\
\text { FC }\end{array}$ & Main Function & $\begin{array}{l}\text { RPKM } \\
\text { C }\end{array}$ & $\begin{array}{c}\text { RPKM } \\
\text { M2 }\end{array}$ \\
\hline hbegfb & $\begin{array}{l}\text { Heparin-binding } \\
\text { EGF-like growth } \\
\text { factor b }\end{array}$ & 00014699 & 4.2 & $\begin{array}{l}\text { Soluble and membrane bound forms. Growth factor in } \\
\text { early stages of wound healing. Promotes dermal repair, } \\
\text { angiogenesis and is expressed by anti-inflammatory } \\
\text { macrophages }[71,72] \text {. }\end{array}$ & 7.8 & 129.2 \\
\hline angpt/4 & Angiopoietin-like 4 & $\begin{array}{l}00035942 \\
00049924\end{array}$ & $\begin{array}{l}4.1 \\
3.9\end{array}$ & $\begin{array}{l}\text { Downregulated by TLR-stimulation in macrophages, } \\
\text { prevents the formation of lipid-laden giant cells }[73] \text { and } \\
\text { associated with anti-inflammatory macrophages }[74,75] \text {. }\end{array}$ & $\begin{array}{l}20.1 \\
20.6\end{array}$ & $\begin{array}{l}260.6 \\
244.3\end{array}$ \\
\hline steap4 & $\begin{array}{l}\text { Six- } \\
\text { transmembrane } \\
\text { epithelial antigen } \\
\text { of prostate } 4\end{array}$ & 00042005 & 3.8 & $\begin{array}{l}\text { Metalloreductase involved in the transfer of ions from } \mathrm{Fe}_{3}{ }^{+} \\
\text {and } \mathrm{Cu}_{2}{ }^{+} \text {to } \mathrm{NAD} \text { and plays a role in cellular homeostasis } \\
\text { during inflammation. Increased Steap4 may reduce } \\
\text { circulating iron available for parasites [25]. }\end{array}$ & 6.8 & 100.6 \\
\hline $\begin{array}{l}\text { ppap } 2 b \\
\text { or plpp3 }\end{array}$ & $\begin{array}{l}\text { Phosphatidic acid } \\
\text { phosphatase type } \\
\text { 2B/ Phospholipid } \\
\text { phosphatase } 3\end{array}$ & $\begin{array}{l}00003642 \\
00045370\end{array}$ & $\begin{array}{l}3.8 \\
3.7\end{array}$ & $\begin{array}{l}\text { Lysophosphatidic acid (LPA) inhibitor. Induced by VEGF } \\
\text { and involved in angiogenesis }[76] \text { and favors anti- } \\
\text { inflammatory phenotypes }[77,78] \text {. }\end{array}$ & $\begin{array}{c}8.7 \\
20.8\end{array}$ & $\begin{array}{l}100.2 \\
222.4\end{array}$ \\
\hline$t d h$ & $\begin{array}{l}\text { L-threonine } \\
\text { dehydrogenase }\end{array}$ & 00008269 & 3.8 & $\begin{array}{l}\text { Converts L-theonine into glycine. Glycine modulates } \\
\text { macrophage activity, plays a role in preventing pyroptosis } \\
\text { and shows cytoprotective effects under hypoxia and } \\
\text { oxidant injury }[38,39] \text {. }\end{array}$ & 21.6 & 286.4 \\
\hline $\operatorname{tgm} 1 / 1$ & $\begin{array}{l}\text { Transglutaminase } \\
\text { 1-like } 1\end{array}$ & 00018981 & 3.8 & $\begin{array}{l}\text { Tgm1 is a cross-linking enzyme involved in tissue } \\
\text { regeneration. Upregulated in macrophages in response } \\
\text { to M-CSF [55]. }\end{array}$ & 45.6 & 509.5 \\
\hline crema & $\begin{array}{l}\text { cAMP-responsive } \\
\text { element } \\
\text { modulator a }\end{array}$ & 00009477 & 3.7 & $\begin{array}{l}\text { Involved in cAMP signaling. Binds cAMP response } \\
\text { element and different splice variants act as both } \\
\text { enhancers and repressors of transcription [79]. }\end{array}$ & 10.0 & 108.6 \\
\hline cremb & $\begin{array}{l}\text { cAMP-responsive } \\
\text { element } \\
\text { modulator b }\end{array}$ & 00033214 & 3.6 & $\begin{array}{l}\text { Involved in cAMP signaling. Binds cAMP response } \\
\text { element and different splice variants act as both } \\
\text { enhancers and repressors of transcription [79]. }\end{array}$ & 15.6 & 164.5 \\
\hline shc2 & $\begin{array}{l}\text { SHC-transforming } \\
\text { protein } 2\end{array}$ & 00020157 & 3.6 & $\begin{array}{l}\text { Mediator of certain growth-factor signaling cascades. } \\
\text { Implicated in cellular proliferation, differentiation, survival } \\
\text { and migration [49]. }\end{array}$ & 5.7 & 58.8 \\
\hline
\end{tabular}

[1] Mills et al., 2000, [15] Mantovani et al., 2004, [23] Wojno et al. 2019, [24] Mantovani et al., 2019, [25] Scarl et al, 2017, [26] Mazzon et al., 2012, [27] Badolato et al., 1994, [28] He et al., 2009, [29] Martinez et al., 2006, [30] Jablonski et al., 2015, [31] Zhang 2002, [32] Clemmensen et al., 2012, [33] Alder et al.,2018, [34] Lahiri et al., 2017, [35] Nathan et al., 1991, [36] Sekelova et al., 2017, [37] Beyer et al., 2012, [38] Carmans et al., 2010, [39] Loomis et al., 2019, [40] Van den Bossche et al., 2017, [41] O'Neill et al., 2019, [42] Li et al., 2007, [43] Oleksiewicz et al., 2011, [44] Abdelkhalek et al., 2009, [45] Deng et al., 2013, [46] de Oliveira et al., 2013, [47] Fox et al., 1995, [48] Walsh and Choi, 2014, [49] Ahmed and Prigent, 2017.

and M2 profiles studied here are distinct from each other and show upregulation of genes associated with M1 and M2 transcriptional profiles in mammals.

\section{Transcriptional profiles of M1 and M2 macrophages are enhanced by T-helper cell associated cytokines}

Mammalian IFN- $\gamma$ is known to activate pro-inflammatory (M1) functions of macrophages, especially when macrophages are co-stimulated with potent microbial stimuli such as LPS. Mammalian IL-4 is known to activate anti-inflammatory (M2) functions of macrophages, also when administered without co-stimuli. We studied enhancement of macrophage function by carp Ifn- $\gamma$ in a co-stimulation experiment 
with LPS by comparing transcription profiles with, and without the presence of carp Ifn- $\gamma$. The majority of differentially expressed genes overlapped between both groups, representing almost $90 \%$ of genes in LPS-only stimulated macrophages and almost $70 \%$ in LPS + Ifn- $\gamma$ stimulated-macrophages (Supplementary figure 3.1A). Similar percentages were found for both, up- and downregulated genes (Supplementary figures 3.1B and 3.1C). Many of the overlapping and most-upregulated genes were even higher expressed in macrophages stimulated with the combination of LPS and Ifn- $\gamma$ (Supplementary table 3.1), suggesting that Ifn- $\gamma$ enhances the gene profile already induced by LPS alone. Indeed, all genes listed in the top 20 except olfm 4 and mecr, showed higher fold-changes in co-stimulated macrophages. Also, several genes of interest upregulated in macrophages stimulated with LPS alone but below the arbitrary threshold of 50 RPKM, such as mhc2dbb, mpeg1.2, and tmem238, were now among the top 20 upregulated genes. Together, these results indicate that Ifn- $\gamma$ can enhance the pro-inflammatory profile induced by LPS alone while retaining the conserved M1-like marker profile.

We also set out to determine the effect of a carp Il-4/13 paralog on carp macrophages by comparing the induced transcription profile with the one of unstimulated macrophages (to ultimately compare with cAMP-stimulated macrophages) but could not detect consistent transcriptional changes different from those in unstimulated control macrophages. The unresponsiveness of carp macrophages to Il-4/13 was not due to lack of bioactivity of the recombinant Il-4/13b1 which was confirmed by a dose-dependent downregulation of pro-inflammatory responses induced in mid-kidney leukocytes (Supplementary figure 3.2); a result similar to what has been observed in grass carp [17]. The unresponsiveness of carp macrophages to Il-4/13 was likely not due to lack of an Il-4/13 sensitive receptor complex on unstimulated macrophages, because the presence of receptors and transcription factors likely involved in Il-4-induced signaling could be identified in unstimulated carp macrophages. These were identified based on known receptor complexes in mammals and on published homologs in zebrafish [80] and grass carp [81] and included a putative Il-4R $\alpha$ chain, two putative paralogs of the IL-13R $\alpha 1$ chain, two putative paralogs of the IL-13R $\alpha 2$ chain and three putative paralogs of the common gamma chain $\gamma$ c. All receptors were expressed at substantial levels of 30-600 RPKM (Supplementary table 3.2.) in unstimulated (control) macrophages. In addition, we could confirm expression of both stat6 and stat3 downstream transcription factors at values of 20-100 RKPM (Supplementary table 3.2) in 
unstimulated (control) macrophages. These results show that the main signaling components of the Il-4 pathway are present and expressed in carp macrophages. Overall, our results indicate that further research into the function of all Il-4/13 paralogs carp needs to be performed before a statement can be made on the ability of carp Il-4/13 to induce an anti-inflammatory (M2-like) profile.

\section{Transcriptional analysis reveals candidate markers to discriminate between M1- and M2-macrophages}

To be able to read-out polarized macrophage responses in future studies in teleost fish, we propose a set of appropriate candidate markers for M1 and M2 macrophages identified in this study for carp. We identified as appropriate candidate markers those genes that are not only significantly regulated or only highly regulated but also sufficiently specific for either M1, or M2 macrophages. First, we determined for all regulated genes their relative expression in M1 and M2 macrophages compared to unstimulated controls (Figure 3.3A). We then determined which genes were significantly regulated only in M1 (red dots), only in M2 (blue dots) or regulated in both M1 and M2 macrophages (blue dots with red edge). We included as appropriate candidate markers those genes significantly up- or downregulated in only one group. Additionally, we included those genes significantly up- or downregulated in both groups, as long as the differences in fold changes are large enough to distinguish between M1 and M2 macrophages. For example, il-1 $\beta$ is significantly upregulated in both M1 and M2 macrophages, but with a fold change of 187.5 (7.55 $\log 2$ fold ) in M1 sufficiently different from the 17 fold change (4.1 log2 fold) in M2 to keep il-1 $\beta$ as an informative marker gene for M1 macrophages. With this in mind, we identified as suitable candidate marker genes those that fit the two following criteria: 1) an expression at least $1.5 \log 2$ fold up- or downregulated compared to unstimulated controls and 2) a ratio of gene expression between M1 and M2 of at least $2.5 \log 2$ fold. The latter means that a potential M1 marker is at least 5.7-fold higher expressed in M1 than in M2 and vice versa.

According to above-described criteria, all genes within the shaded areas of Figure 3.3A are potential marker genes for M1 (red shade) or M2 (blue shade) macrophages (specified in supplementary table 3.3). Of high interest, genes within striped areas represent genes that fit these criteria for both M1 and M2 subsets and are oppositely regulated in M1 versus M2. These genes are therefore among the most specific marker genes (specified in supplementary table 3.3). For six potential 
candidate markers for M1 (il1 $\beta$, nos $2 b$ and saa) and M2 (timp2b, tgm2b and $\arg 2)$ macrophages we validated their suitability for detection by real-time qPCR. We confirmed a significant increase in il1 $\beta$, nos $2 b$ and saa expression in M1 but not in M2 macrophages (Figure 3.3B-D). Likewise, we confirmed a significant increase in timp $2 b$, tgm $2 b$ and $\arg 2$ in M2 macrophages but not in M1 macrophages (Figure 3.3E-G). This suggests these markers, among others, are suitable for gene expression studies on polarized macrophages populations. It also highlights these genes as valuable targets for additional approaches such as the development of specific antibodies or the generation of zebrafish transgenic reporter lines which would both allow to study macrophage polarization at the cellular level, if not in vivo.
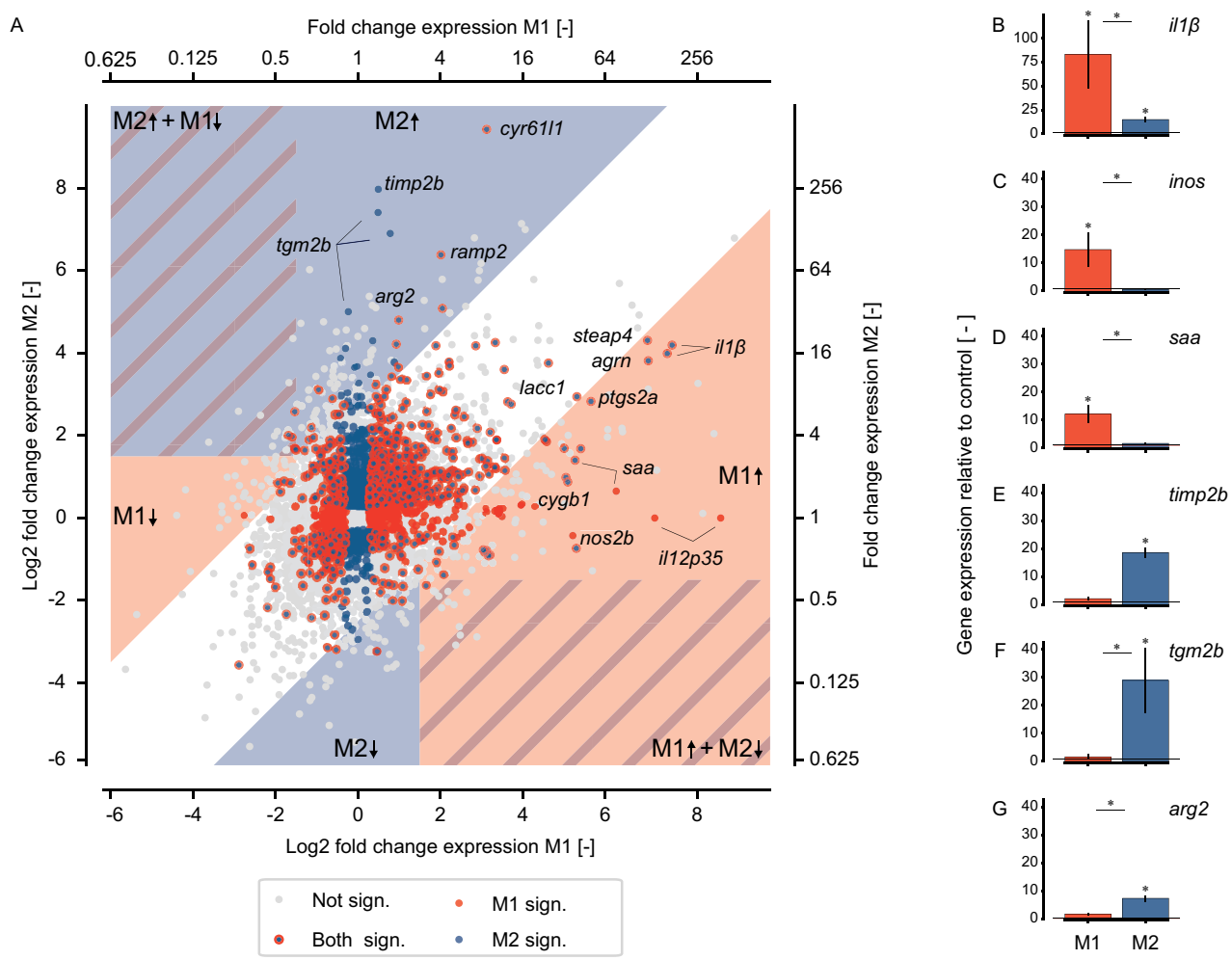

Figure 3.3 Graphical representation of transcriptional data reveals candidate markers for M1 and M2 macrophages in carp. A) Graphical representation of transcriptional profiles of carp macrophages polarized for $6 \mathrm{~h}$ with $30 \mu \mathrm{g} / \mathrm{ml}$ LPS (M1) or $0.5 \mu \mathrm{g} / \mathrm{ml}$ cAMP (M2) compared to unpolarized control macrophages. Dots represent genes with an average number of reads $>50$ reads per kilobasepair per million reads (RPKM) in either stimulated or unstimulated control macrophages. Grey dots indicate genes that are not significantly regulated in either M1 or M2 macrophages $(p>0.05)$. Red dots indicate 
genes that are significantly regulated in M1 macrophages $(p<0.05)$. Blue dots indicate genes that are significantly regulated in M2 macrophages $(p<0.05)$. Blue dots with red edges indicate genes that are significantly regulated in M1 and M2 macrophages $(p<0.05)$. Position on the $x$-axis represents the average log2 fold change (bottom axis) or fold change (top axis) of LPS stimulated macrophages compared to unstimulated controls. Position on the $y$-axis displays the average log2 fold change (left axis) or fold change (right axis) of cAMP stimulated macrophages compared to unstimulated controls. Dots within the translucent area represent potential marker genes that change at least 1.5 log2 fold from unstimulated controls ( $\log 2$ fold $<-1.5 \log 2$ fold $>1.5$ ). Dots in the red translucent area represent genes that are at least $2.5 \log 2$ fold higher or lower expressed in M1 macrophages then M2 macrophages. Dots within the blue translucent area represent genes that are at least $2.5 \log 2$ fold higher or lower expressed in M2 macrophages then M1 macrophages. Dots in the striped translucent area represent potential marker genes that inversely regulated in M1 and M2 macrophages. Labels with arrows indicate whether genes are up- or downregulated. Examples of good potential marker genes are labeled. Additional marker candidates are included as supplementary data (Supplementary table 3). Data are of $n=3$ fish.

Real-time gene expression of il1 $\beta(\mathbf{B})$, inos (nos $2 b)(\mathbf{C})$, saa (D), timp $2 b(\mathbf{E}), \operatorname{tgm} 2 b(\mathbf{F})$ and $\arg 2(\mathbf{G})$ using common primers for paralog sequences confirms these genes as appropriate markers for polarized macrophages. Gene expression was normalized to the $s 11$ protein of the $40 s$ subunit as a reference gene and shown as the fold change relative to the unstimulated controls (line at $y=0$ ). Data are the mean and standard deviation of $n=4$. Data were analyzed using a repeated measures ANOVA with Dunnett's $\mathrm{T} 3$ post-hoc tests for unequal variances or the Kruskal-Wallis test in case normality was violated. Differences were considered significant when $p<0.05(*)$.

\section{Discussion}

In this study we performed a comprehensive analysis of the transcriptional profile of M1- and M2-like polarized macrophages from a teleost fish and compared the genes highest expressed with those known for mammalian M1 and M2 counterparts. We used LPS and cAMP as main stimuli and first confirmed M1- and M2-like functional phenotypes of macrophages from common carp, which were subsequently used for RNA sequencing. The resulting transcriptional profiles of carp macrophages show a high degree of conservation with those of polarized macrophages as we know them today from humans and mice. These profiles provide an unbiased and solid framework to not only confirm previously used markers but select additional markers of polarized macrophage responses in a non-mammalian species.

The classical approach of using cytokine stimuli to polarize mammalian macrophages may not necessarily be directly applicable, nor needed, for studies on fish macrophages. Macrophages of mice and humans have traditionally been polarized with microbial stimuli such as LPS combined with cytokines associated with Th1 (IFN- $\gamma$ ) for M1 macrophages and have traditionally been polarized with cytokines associated with Th2 responses (IL-4) for M2 [82, 83]. Furthermore, addition of the growth factors GM-CSF or M-CSF help to induce polarization towards M1 or M2 phenotypes, respectively [84]. For studies on fish macrophages it is not always possible nor evident to copy these exact experimental set-ups. 
In fish, the degree of functional conservation of the cytokines IFN- $\gamma$ and IL-4 remains subject of discussion $[85,86]$, with evidence of their ability to induce polarized $\mathrm{T}$ cell-mediated responses being stronger for IFN- $\gamma$ [87] than for IL-4 $[88,89]$. Moreover, although the presence of M-CSF has been studied at expression level[90] and effects on macrophage proliferation have been reported[91], evidence of the presence of GM-CSF in fish genomes remains elusive [92, 93]. It has been shown that macrophages of goldfish and carp can be stimulated with LPS [8] alone to induce M1-like phenotypes producing nitric oxide, or with cAMP $[8,16]$ to induce M2-like phenotypes displaying arginase activity. Indeed, it is plausible that the initial trigger for macrophage polarization into M1 or M2 could rely primarily on sensing microbial/parasite infection or other innate danger signals, without a required presence of T-cell derived cytokines. This reverts the idea of the dichotomous Th1 and Th2 driving forces by suggesting that polarized innate immune responses could drive polarized adaptive responses, a concept described as 'the macrophages first' hypothesis [4, 12].

We primarily used innate immune stimuli (LPS, cAMP) to stimulate carp macrophages and determine subsequent polarized phenotypes with differences in morphology, function and transcriptional profiles. Importantly, the gene expression profiles of these polarized M1 and M2 macrophages of fish revealed upregulation of many genes also associated with the concurrent phenotypes in mammalian macrophages. Some of these genes have previously been associated with activated macrophages in varying fish species. For example, the chemokine cxcl8l1 (otherwise known as cxca) was mentioned as cxcl8a and specifically expressed in LPSstimulated macrophages of grass carp. We likewise noticed a 16-fold upregulation in carp M1 macrophages. The chemokine $c c l 20 a$ was also mentioned as increased in LPS-stimulated macrophages in grass carp [22] in the same study and was also substantial (8 log2 fold) increase in one ccl20a paralog in carp M1 macrophages, although not among the most highly expressed genes. Chemokine receptors $c x c r 3$ were mentioned as markers of M1 (cxcr3.1) and M2 (cxcr3.2) macrophages of grass carp, ayu (Plecoglossus altivelis) and spotted green pufferfish (Tetraodon nigroviridis) [21]. We likewise noticed an upregulation of $c x c r 3.3$, which is closely related to cxcr3.1, in carp M1 macrophages, but were unable to confirm upregulation of cxcr3.2 in carp M2 macrophages. The pro-inflammatory cytokines il1 $\beta$ and il6 were mentioned as highly expressed in $m p e g 1^{+} \mathrm{M} 1\left(\operatorname{tnf1^{+}}\right)$ macrophage subsets of zebrafish, and the chemokine receptor $c x c r 4 b$ and alox $5 a p$, required for leukotriene 
synthesis, mentioned as highly expressed in M2 (mpeg $1^{+} /$tnf1 $1^{-}$) macrophage subsets [20]. We likewise noticed an increase of il1 $\beta$ and il6 in carp M1 macrophages and of $c x c r 4 b$ and alox5ap in M2 carp macrophages. Among other M1 markers, the chemokine cxcl11 was mentioned as upregulated after mycobacterial infection in zebrafish larvae [20]. This typical M1 marker showed a strong decrease of expression in carp M2 macrophages. In summary, our data provides a combination of functional and comprehensive, unbiased transcriptional information on fish macrophages polarized towards both M1 and M2 polarization states. Our gene expression profiles on carp macrophages unite several observations of others on macrophages from different fish species. Our data not only indicate that the distinct gene expression profiles of carp macrophages are indeed distinct M1- and M2-like profiles but also highlight M1- and M2-specific gene transcription profiles show a striking conservation from teleost fish to mammals.

Our data suggest that carp macrophages could be polarized by innate damage and danger signals without the presence of T-cell derived cytokines and thus provide support to the 'macrophages first' point of view [12]. As mentioned before, for studies in fish it is not always evident to copy the common practice of costimulating macrophages with the cytokines IFN- $\gamma$ and IL-4 to polarize into M1 or M2 states. Still, it remains of interest to study the effect of these cytokines on fish macrophages, primarily to investigate evolutionary conservation of cytokine function. Although co-stimulation of carp macrophages with recombinant carp Ifn- $\gamma$ upregulated genes additional to those upregulated by LPS alone, the major effect of Ifn- $\gamma$ was an amplification of the expression of the majority of the genes also upregulated by LPS stimulation alone. This hints at a certain degree of conservation of function for Ifn- $\gamma$ with respect to macrophage activation. In contrast to observations in other teleost species [16, 17], we could detect no effect of carp Il-4/13b1 on macrophages, despite evident bioactivity of the recombinant protein. The presence in carp macrophages of a putative Il-4/13 sensitive receptor complex and the machinery for Il-4/13 signaling suggests there could be subfunctionalization in function or target [94] between different Il-4/13 paralogs. Preliminary analysis of the common carp genome revealed genes encoding at least four different 1l-4/13 paralogs. Without studying the biological effect of all cytokine and receptor paralogs on fish macrophages it is difficult to draw conclusions on the exact role and effect of these Il-4/13-like cytokines on fish macrophages. 
Here, we provided a comprehensive list of candidate marker genes to help identify M1 and M2 fish macrophages. First, we could confirm using qPCR clear differences in gene expression between a number of well-known markers for M1 and M2 macrophages, including il1 $\beta$, inos (nos $2 b$ ), and saa for M1 macrophages and timp $2 b$, $\operatorname{tgm} 2 b$ and $\arg 2$ for M2 macrophages. These markers may be informative, but they are not always exclusive. For example, although il $1 \beta$ is much higher expressed in M1 macrophages, it is not absent in M2 macrophages. Moreover, expression of $\operatorname{tnf} \alpha$, which is commonly used to visualize inflammatory macrophages in transgenic zebrafish, is upregulated in carp M1 macrophages and downregulated in M2 macrophages as expected. However, differences in expression are small compared to other genes and it is readily detectable in M1, M2 and control macrophages (15180 RPKM). Such observations indicate that other candidate markers may be even more suitable because they are more specific for a particular polarization state. Such markers would be up- or downregulated compared to controls in a specific macrophage subset and either remain the same or show opposite regulation in the other subset. We therefore set stringent thresholds for regulation and differences between subsets to provide more selective lists of candidate marker genes. For M1 macrophages, interesting additional candidate markers could include heat-shock protein 70 ( $h s p 70$ ), as many hsp70 paralogs are upregulated in M1 macrophages while they are slightly downregulated in M2 macrophages. In mammals, HSP70 prevents NO-induced apoptosis in macrophages $[95,96]$, indicating its functional significance in inflammatory macrophages. Hsp70 has also been indicated in antiviral responses in grass carp [97]. Another interesting candidate is irg1, because upregulation of both paralogs is increased to a much higher extent in M1 compared to the upregulation in M2 macrophages and is involved in the metabolic phenotype of these macrophages [40,41]. Particularly interesting is cxcl11, as this traditional human M1 marker is not only a good M1 marker for carp macrophages, but has been indicated as M1 marker in zebrafish as well [20]. For M2 macrophages, interesting additional markers could include the mannose receptor c type $1 b$ ( $m r c 1 b$ ) genes which are upregulated in M2 but downregulated in M1 macrophages. Indeed, the mannose receptor has been described and used as a M2 marker for human and murine M2 macrophages [83, 98]. Furthermore, angiopoietin-like 4 (angptl4) appears consistently upregulated in M2 macrophages only and is associated with M2 macrophage polarization and tissue repair in mammals [75]. For macrophages of mammalian species it is becoming clear that subtle differences in polarization states exist between similar but distinct stimuli, both in vitro and in vivo [2, 37]. 
This advocates the use of a comprehensive set of markers as opposed to a single gene to discriminate between polarization states. The debate continues on how well cytokine-dependent in vitro phenotypes reflect those in vivo [1,52] and may find contributions from studies in model animals. We have previously discussed the relevance of studying macrophage behavior in vivo and argued that candidate markers from carp could aid the development of new M1 and M2 transgenic zebrafish lines [12]. Transgenic zebrafish, well known for the possibility to visualize and follow specific immune cells in vivo $[99,100]$ may be of help when M1- and M2like macrophages can be traced real time.

Last but not least, steering innate immune responses could provide a valuable alternative to the use of antibiotics and could replace or at least help vaccination in the quest to sustainably improve fish health in aquaculture, a form of animal production which is rapidly becoming more important [10]. The development of simple read-out systems can be crucial to the development of targeted innate immune stimulants that are able to steer macrophages towards the polarization state that is most effective against the pathogen at hand. In this study, we provide both transcriptional profiles and potential markers which will contribute substantially to the development of new read-outs to determine polarization states of the innate immune system.

\section{Materials and methods}

\section{Experimental animals}

European common carp (Cyprinus carpio carpio L.) used in experiments were the 12 months old offspring of a cross between the R3 strain of Polish origin and the R8 strain of Hungarian origin [101]. Carp were bred and reared in the aquatic research facility of Wageningen University and Research at $23^{\circ} \mathrm{C}$ in recirculating UV-treated water and fed pelleted dry food (Skretting, Nutreco) twice daily. All experiments were performed with the approval of the Animal Experiments Committee of Wageningen University and Research (Ethical Committee documentation number 2017.W-0034) in accordance with the guidelines and regulations. 


\section{In vitro culture and polarization of carp macrophages}

Head kidney-derived macrophages were obtained as described previously [8]. In short, total head kidney leukocytes were cultured for 6 days at $27^{\circ} \mathrm{C}$, at a density of $17.5 \times 10^{6}$ cells $/ 75 \mathrm{~cm}^{2}$ flask in complete NMGFL-15 medium (incomplete -NMGFL-15 supplemented with 5\% pooled carp serum (PCS) and 10\% bovine calf serum (Invitrogen Life Technologies) with $100 \mathrm{U} / \mathrm{ml}$ of penicillin, $100 \mu \mathrm{g} / \mathrm{ml}$ of streptomycin sulfate (Gibco) and $50 \mu \mathrm{g} / \mathrm{ml}$ Gentamycin (Sigma Aldrich) to obtain macrophages.

To polarize, macrophages were harvested by gentle scraping after incubation on ice for 15 minutes. Cells were pelleted at $450 \mathrm{xg}$ for $10 \mathrm{~min}$ at $4^{\circ} \mathrm{C}$ before resuspension in cRPMI+ (RPMI 1640 culture medium with $25 \mathrm{mM}$ HEPES and 2mM L-glutamine, supplemented with L-glutamine $(2 \mathrm{mM})$, penicillin $\mathrm{G}(100 \mathrm{U} / \mathrm{ml})$, streptomycin sulfate $(100 \mu \mathrm{g} / \mathrm{ml})$ and heat-inactivated PCS $(1.5 \% \mathrm{v} / \mathrm{v}))$. Depending on the assay, macrophages were polarized for $6 \mathrm{~h}$ or $24 \mathrm{~h}$ with $30 \mu \mathrm{g} / \mathrm{ml}$ LPS (Escherichia coli, L2880, Sigma-Aldrich) with or without $100 \mathrm{ng} / \mathrm{ml}$ recombinant Ifn- $\gamma$ for M1 macrophages, or with $0.5 \mathrm{mg} / \mathrm{ml}$ dibutyryl cAMP $\left(\mathrm{N}^{6}, 2^{2}-\mathrm{O}\right.$-dibutyryladenosine 3':5'-cyclic monophosphate sodium D0627, Sigma Aldrich, referred to as cAMP) or $100 \mathrm{ng} / \mathrm{ml}$ recombinant Il-4/13b1 for M2 macrophages, or with an equal volume of medium as unstimulated controls. Cells were cultured at $27^{\circ} \mathrm{C}$ in the presence of $5 \% \mathrm{CO}_{2}$. For analysis of phagocytosis, macrophages were polarized using the same medium and stimuli as described above, but kept in the culture flasks until harvested directly before use at day 7. After harvest, cells were resuspended in cRPMI without PCS before use in phagocytosis assays.

\section{Functional and morphological confirmation of macrophage polarization}

NO production was determined in culture supernatants of polarized macrophages. In brief, $5 \times 10^{5}$ macrophages per well were seeded in 96-wells plates (Corning) in $150 \mu \mathrm{l}$ of cRPMI+. After polarization, NO production was determined as nitrite in 75 $\mu \mathrm{l}$ culture supernatant as described previously [102].

Arginase activity was measured in cell lysates as the amount of urea produced by the conversion of L-arginine to urea by arginase and normalized using a ratio of the sample protein content compared to lysate of control cells. A total of $1.5 \times 10^{6}$ cells polarized for $24 \mathrm{~h}$ in $450 \mu \mathrm{l} \mathrm{cRPMI+}$, were lysed in $100 \mu \mathrm{l}$ of $0.1 \%$ Triton X-100. Protein content of the samples was determined using the Bradford protein dye 
reagent (Bio-Rad) according to the manufacturer's protocol. Arginase activity was measured in $25 \mu$ l lysate as described previously for $50 \mu$ lysate [8], but volumes were scaled down accordingly. Arginase activity was determined as the conversion of L-arginine to urea by arginase and expressed in $\mathrm{nmol} / \mathrm{min} / 10^{6}$ cells.

For brightfield microscope images, $5 \times 10^{4}$ macrophages polarized for $24 \mathrm{~h}$ in 150 $\mu \mathrm{l}$ cRPMI+ in 96-wells plates (Corning) were imaged using a DMi8 inverted digital microscope (Leica Microsystems), controlled by Leica LASX software (version 3.4.2.) and equipped with 40x (NA 0.6) and 20x (NA 0.4) long distance objectives (Leica Microsystems).

\section{Recombinant cytokines}

Recombinant carp interferon gamma 2 (Ifn- $\gamma$ ) was produced as described previously [14]. Protein analysis by SDS-PAGE (12\% Tris-HCl, BioRad) stained with GelCode ${ }^{\circ}$ Blue Stain Reagent (Thermo scientific) revealed proteins were at least 95\% pure and the chromogenic Limulus amebocyte lysate end-point test (Charles River Laboratories) showed that the residual endotoxin content was below detection limit $(<0.15 \mathrm{EU})$

Recombinant carp Il-4/13b1 (previously named Il-4/13B) was produced essentially as described previously [88] and the expression plasmid [88] a kind gift of Professor T. Moritomo and Dr. F. Katakura, Laboratory of Comparative Immunology, Nihon University. In short, the poly-His-tagged Il-4/13b1 protein was expressed in Rosettagami B (DE3) pLysS Competent cells (Novagen) and purified using sepharose beads (Qiagen) followed by gel chromatography size exclusion using Superdex ${ }^{\mathrm{TM}} 200$ prepgrade 26/600 column (GE Heathcare). Protein analysis by SDS-PAGE (12\% Tris$\mathrm{HCl}$, BioRad) stained with GelCode ${ }^{\star}$ Blue Stain Reagent (Thermo scientific) revealed that proteins were at least $95 \%$ pure and residual endotoxin content was shown to be $<0.005$ EU/ml (EndoZyme ${ }^{\circledR}$ II Recombinant Factor C (rFC) Assay, Hyglos GmbH).

\section{Phagocytosis}

Phagocytic compartment (\% of phagocytic cells) and phagocytic capacity of polarized macrophages was determined by flow cytometry as described by Petit et al.[94] using fluorescent beads of 1 or $2 \mu \mathrm{m}$ (PSF-001UM and PSF-002UM Red, MagSphere). Phagocytosis was quantified using a CytoFLEX LX Flow Cytometer (Beckman-Coulter). Data were analyzed using FlowJo v10 (BD Biosciences). 
Phagocytic activity was calculated as the relative proportion of total cells that ingested at least one bead. Phagocytic capacity was calculated as the relative proportion of total cells that ingested $1,2,3,4, \geqslant 5$ beads for $1 \mu \mathrm{m}$ beads or $1,2,3,4$, $5, \geqslant 6$ beads for $2 \mu \mathrm{m}$ beads.

For fluorescent images, $1 \times 10^{5}$ polarized macrophages were incubated in 8-well $\mu$-Slide (ibiTreat 80826, Ibidi) with fluorescent beads of $1 \mu \mathrm{m}$ (PSF-001UM Red, MagSphere) in a $1 / 10$ cell/bead ratio, for $120 \mathrm{~min}$ at $27^{\circ} \mathrm{C}$ in the presence of $5 \% \mathrm{CO}_{2}$. Cells were then washed with Hanks balanced salt solution (HBSS) (without glucose, Ca, phenol red (D8537 Sigma)), fixed with 4\% formaldehyde pH 7.4 (Formaldehyde solution (w/v) Methanol free, Thermo Scientific) in HBSS for 8 min. at RT and washed again three times with HBSS. Cell membranes were labelled with $8 \mu \mathrm{g} / \mathrm{ml}$ wheat germ agglutinin (WGA) Alexa Fluor 488 conjugate (Thermo Fisher) in HBSS + Ca + Mg (D8662 Sigma) for $30 \mathrm{~min}$. at RT in the dark. Cells were washed three times with HBSS and nuclei were stained with 4',6-Diamidino-2-phenylindole dihydrochloride (DAPI, D1306, Invitrogen) for $10 \mathrm{~min}$, at RT in the dark. Cells were washed three times with HBSS and imaged using a DMi8 inverted digital microscope (Leica Microsystems), controlled by Leica LASX software (version 3.4.2.) and equipped with 40x (NA 0.6) and 20x (NA 0.4) long distance objectives (Leica Microsystems).

\section{RNA extraction}

Extracted RNA was used for Illumina sequencing and RT-qPCR experiments. For this, $1.5 \times 10^{6}$ macrophages were polarized in 24-well plates (Corning) in a total volume of $450 \mu \mathrm{l} /$ well and stimulated for $6 \mathrm{~h}$ before RNA extraction. Technical replicates were pooled, and total RNA was extracted from $3 \times 10^{6}$ cells using the Rneasy mini kit (Qiagen) according to the manufacturer's protocol including the on-column DNase digestion using the RNase-free DNase digestion kit (Qiagen). RNA was stored at $-80^{\circ} \mathrm{C}$ until use for sequencing and $\mathrm{qPCR}$ experiments.

\section{Illumina sequencing and sequencing analysis}

Quality, integrity and quantity of the RNA was assessed using a Bioanalyzer (Agilent 2100 total RNA Nano series II chip, Agilent). RNAseq libraries were prepared from $0.5 \mu \mathrm{g}$ total RNA using the TruSeq ${ }^{\circledR}$ Stranded mRNA Library Prep kit according to the manufacturer's instructions (Illumina Inc.). All RNAseq libraries were sequenced on an Illumina HiSeq2500 sequencer as $1 \times 50$ nucleotides single-end 
reads according to Kolder et al. [103] and Petit et al. [104]. The Illumina pipeline was used for image analysis and base calling. Reads were aligned to the genome assembly of common carp (BioProject: PRJNA73579) [103]. Secondary alignments of reads were excluded by filtering the files using SAMtools (version 0.1.18) [105]. Aligned fragments per predicted gene were counted from SAM alignment files using the Python package HTSeq (version 0.5.3p9) [106].

\section{Differential gene expression}

Differential gene expression was analyzed using the bioinformatics package DESeq 2.0 (v1.22.2) and R statistical software (3.5.5) [107]. Statistical analysis was performed using a paired design with unstimulated cells as control and performed for LPS, cAMP, LPS + IFN- $\gamma$ and Il-4/13b1 stimulated macrophages independently ( $n=3$ independent cultures for each stimulus). The paired design allowed for a better comparison between independent cultures, reducing noise generated by independent culture to culture differences. Within DESeq 2.0, $p$-values were adjusted using Benjamini \& Hochberg corrections for controlling false discovery rate and results were considered statistically significant when $p_{\text {adjusted }} \leqslant 0.05$. Additional subsetting and analysis was performed based on the log2 fold change (DESeq 2.0) and the number of reads per kilobasepair per million reads (RPKM). Proportional Venn diagrams were generated using the VennDiagram package [108] (1.6.20) in R statistical software (3.5.5). Candidate markers were determined as specified in the supplementary methods.

\section{Real-time Quantitative PCR}

RT-qPCR analysis was performed with a Rotor-Gene 6000 (Corbett Research) using ABsolute qPCR SYBR Green Mix (Thermo Scientific). The primers used are shown in Supplementary table 3.4. Fluorescence data from RT-qPCR experiments were analyzed using Rotor-Gene Analysis software (v1.7). The take-off value for each sample and the average reaction efficiencies $(E)$ for each primer set were obtained upon Comparative Quantitation Analysis from Rotor Gene Software [109]. The relative expression ratio $(R)$ of a target gene was calculated based on the average $E$ and the take-off deviation of sample versus control and expressed relative to the $s 11$ protein of the 40 s subunit as a reference gene. 


\section{Statistical analysis}

Raw data of technical replicates were averaged per individual before statistical analysis was performed using IBM ${ }^{\oplus}$ SPSS ${ }^{\oplus}$ Statistics 26 . For RT-qPCR data, statistical analysis was performed on log-transformed data to obtain normal distributions. Significant differences between groups were determined using a (repeated measures) one-way analysis of variance (ANOVA) followed by Tukey's post-hoc test for multiple comparisons. In absence of sphericity (Mauchly's Test of Sphericity) in repeated measures ANOVA the Geisser-Greenhouse correction was applied. In case of unequal variances determined by Levene's test, Dunnett's T3 post-hoc test was used for multiple comparisons. In the absence of normality as determined by the Shapiro-Wilk test, the non-parametric Friedman's two-way ANOVA by ranks was used for paired analysis and the Kruskal-Wallis test was used for independent samples.

\section{Acknowledgements}

The authors gratefully acknowledge Professor T. Moritomo and Dr. F. Katakura for the expression plasmid encoding recombinant Il-4/13b1 and for their protocols for recombinant protein expression. The authors gratefully acknowledge Willy van den Berg and Mirelle Kleuskens for their contribution in the production of recombinant Il-4/13b1, Edwin Tijhaar for the production of recombinant Ifn- $\gamma$ and Ben Meijer for his assistance in measuring phagocytosis.

\section{Author contribution statement}

GFW and HPS acquired funding, initiated and conceptualized the study. ASW, IRF, MF and GFW contributed to the design of the experiments. ASW, IRF, JP and MHS performed experiments. ASW and WGvV performed analysis of the data with contribution from MCP, JP and GFW. ASW and GFW drafted the manuscript, ASW prepared the figures and tables with contribution from WGvV and GFW. JP, WGvV, MCP, MF, and HPS critically reviewed the manuscript.

\section{Additional information}

The authors declare no competing interests. 


\section{Funding}

This work was supported by the European Commission under the 8th (H2020) Framework Program for Research and Technological Development of the European Union (PARAFISHCONTROL Grant No. 634429) and by the 7th Framework program [NEMO Grant No. PITN-GA-2008-214505]. This publication reflects the views only of the authors, and the European Commission cannot be held responsible for any use which may be made of the information.

\section{Data availability statement}

The datasets analyzed during the current study are available in the NCBI Sequence Read Archive, BioProject ID: PRJNA627088 (RNA sequencing data). Or available from the corresponding author upon reasonable request (other data). 


\section{References}

1. Mills CD, Kincaid K, Alt JM, Heilman MJ, Hill AM (2000) M-1/M-2 Macrophages and the Th1/ Th2 Paradigm. J Immunol. https://doi.org/10.4049/jimmunol.164.12.6166.

2. Xue J, Schmidt S V, Sander J, Draffehn A, Krebs W, Quester I, De Nardo D, Gohel TD, Emde M, Schmidleithner L, Ganesan H, Nino-Castro A, Mallmann MR, Labzin L, Theis H, Kraut M, Beyer M, Latz E, Freeman TC, Ulas T, Schultze JL (2014) Transcriptome-based network analysis reveals a spectrum model of human macrophage activation. Immunity 40:274-288. doi:10.1016/j. immuni.2014.01.006.

3. Biswas SK, Mantovani A (2010) Macrophage plasticity and interaction with lymphocyte subsets: cancer as a paradigm. Nat Immunol 11:889.

4. Mills CD, Ley K (2014) M1 and M2 Macrophages: The Chicken and the Egg of Immunity. 92037:716-726. doi:10.1159/000364945.

5. Schroder K, Irvine KM, Taylor MS, Bokil NJ, Le Cao K-A, Masterman K-A, Labzin LI, Semple CA, Kapetanovic R, Fairbairn L, Akalin A, Faulkner GJ, Baillie JK, Gongora M, Daub CO, Kawaji H, McLachlan GJ, Goldman N, Grimmond SM, Carninci P, Suzuki H, Hayashizaki Y, Lenhard B, Hume DA, Sweet MJ (2012) Conservation and divergence in Toll-like receptor 4-regulated gene expression in primary human versus mouse macrophages. Proc Natl Acad Sci U S A 109:E944E953. doi:10.1073/pnas.1110156109.

6. Shay T, Jojic V, Zuk O, Rothamel K, Puyraimond-Zemmour D, Feng T, Wakamatsu E, Benoist C, Koller D, Regev A, Consortium I (2013) Conservation and divergence in the transcriptional programs of the human and mouse immune systems. Proc Natl Acad Sci U S A 110:2946-2951. doi:10.1073/pnas.1222738110.

7. Spiller KL, Wrona EA, Romero-Torres S, Pallotta I, Graney PL, Witherel CE, Panicker LM, Feldman RA, Urbanska AM, Santambrogio L, Vunjak-Novakovic G, Freytes DO (2016) Differential gene expression in human, murine, and cell line-derived macrophages upon polarization. Exp Cell Res 347:1-13. doi:10.1016/j.yexcr.2015.10.017.

8. Joerink M, Ribeiro CMS, Stet RJM, Hermsen T, Savelkoul HFJ, Wiegertjes GF (2006) Head Kidney-Derived Macrophages of Common Carp (Cyprinus carpio L.) Show Plasticity and Functional Polarization upon Differential Stimulation. I Immunol 177:61-69. doi:10.4049/ jimmunol.177.1.61.

9. Joerink M, Forlenza M, Ribeiro CMS, de Vries BJ, Savelkoul HFJ, Wiegertjes GF (2006) Differential macrophage polarisation during parasitic infections in common carp (Cyprinus carpio L.). Fish Shellfish Immunol 21:561-571. doi:https://doi.org/10.1016/j.fsi.2006.03.006.

10. FAO (2018) The State of Fisheries and Aquaculture in the world 2018.

11. Forlenza M, Fink IR, Raes G, Wiegertjes GF (2011) Heterogeneity of macrophage activation in fish. Dev Comp Immunol 35:1246-1255. doi:10.1016/j.dci.2011.03.008.

12. Wiegertjes GF, Wentzel AS, Spaink HP, Elks PM, Fink IR (2016) Polarization of immune responses in fish: The 'macrophages first' point of view. Mol Immunol 69:146-156. doi:10.1016/j. molimm.2015.09.026.

13. Grayfer L, Kerimoglu B, Yaparla A, Hodgkinson JW, Xie J, Belosevic M (2018) Mechanisms of Fish Macrophage Antimicrobial Immunity. Front Immunol 9:1105. doi:10.3389/fimmu.2018.01105. 
14. Arts JAJ, Tijhaar EJ, Chadzinska M, Savelkoul HFJ, Verburg-van Kemenade BML (2010) Functional analysis of carp interferon- $\gamma$ : Evolutionary conservation of classical phagocyte activation. Fish Shellfish Immunol 29:793-802. doi:10.1016/J.FSI.2010.07.010.

15. Mantovani A, Sica A, Sozzani S, Allavena P, Vecchi A, Locati M (2004) The chemokine system in diverse forms of macrophage activation and polarization. Trends Immunol 25:677-686. doi:10.1016/j.it.2004.09.015.

16. Hodgkinson JW, Fibke C, Belosevic M (2017) Recombinant IL-4/13A and IL-4/13B induce arginase activity and down-regulate nitric oxide response of primary goldfish (Carassius auratus L.) macrophages. Dev Comp Immunol 67:377-384. doi:10.1016/j.dci.2016.08.014.

17. Yang Z-J, Li C-H, Chen J, Zhang H, Li M-Y, Chen J (2016) Molecular characterization of an interleukin-4/13B homolog in grass carp (Ctenopharyngodon idella) and its role in fish against Aeromonas hydrophila infection. Fish Shellfish Immunol 57:136-147. doi:10.1016/j. fsi.2016.08.022.

18. Chang C-I, Zoghi B, Liao JC, Kuo L (2000) The Involvement of Tyrosine Kinases, Cyclic AMP/ Protein Kinase A, and p38 Mitogen-Activated Protein Kinase in IL-13-Mediated Arginase I Induction in Macrophages: Its Implications in IL-13-Inhibited Nitric Oxide Production. J Immunol 165:2134 LP - 2141. doi:10.4049/jimmunol.165.4.2134.

19. Nguyen-Chi M, Laplace-Builhe B, Travnickova J, Luz-Crawford P, Tejedor G, Phan QT, DurouxRichard I, Levraud JP, Kissa K, Lutfalla G, Jorgensen C, Djouad F (2015) Identification of polarized macrophage subsets in zebrafish. Elife 4:1-14. doi:10.7554/eLife.07288.

20. Rougeot J, Torraca V, Zakrzewska A, Kanwal Z, Jansen H, Spaink HP, Meijer AH (2019) RNAseq profiling of leukocyte populations in zebrafish larvae reveals a cxcl11 chemokine gene as a marker of macrophage polarization during mycobacterial infection. bioRxiv 554808 . doi:10.1101/554808.

21. Lu X-J, Chen Q, Rong Y-J, Chen F, Chen J (2017) CXCR3.1 and CXCR3.2 Differentially Contribute to Macrophage Polarization in Teleost Fish. J Immunol 198:4692 LP - 4706. doi:10.4049/ jimmunol.1700101.

22. Hu Y, Wei X, Liao Z, Gao Y, Liu X, Su J, Yuan G (2018) Transcriptome Analysis Provides Insights into the Markers of Resting and LPS-Activated Macrophages in Grass Carp (Ctenopharyngodon idella). Int. J. Mol. Sci. 19.

23. Tait Wojno ED, Hunter CA, Stumhofer JS (2019) The Immunobiology of the Interleukin-12 Family: Room for Discovery. Immunity 50:851-870. doi:10.1016/j.immuni.2019.03.011.

24. Mantovani A, Dinarello CA, Molgora M, Garlanda C (2019) Interleukin-1 and Related Cytokines in the Regulation of Inflammation and Immunity. Immunity 50:778-795. doi:10.1016/j. immuni.2019.03.012.

25. Scarl RT, Lawrence CM, Gordon HM, Nunemaker CS (2017) STEAP4: its emerging role in metabolism and homeostasis of cellular iron and copper. J Endocrinol 234:R123-R134. doi:10.1530/JOE-16-0594.

26. Mazzon C, Anselmo A, Soldani C, Cibella J, Ploia C, Moalli F, Burden SJ, Dustin ML, Sarukhan A, Viola A (2012) Agrin is required for survival and function of monocytic cells. Blood 119:55025511. doi:10.1182/blood-2011-09-382812. 
27. Badolato R, Wang JM, Murphy WJ, Lloyd AR, Michiel DF, Bausserman LL, Kelvin DJ, Oppenheim IJ (1994) Serum amyloid A is a chemoattractant: induction of migration, adhesion, and tissue infiltration of monocytes and polymorphonuclear leukocytes. J Exp Med 180:203 LP - 209. doi:10.1084/jem.180.1.203.

28. He RL, Zhou J, Hanson CZ, Chen J, Cheng N, Ye RD (2009) Serum amyloid A induces G-CSF expression and neutrophilia via Toll-like receptor 2. Blood 113:429 LP - 437. doi:10.1182/ blood-2008-03-139923.

29. Martinez FO, Gordon S, Locati M, Mantovani A (2006) Transcriptional Profiling of the Human Monocyte-to-Macrophage Differentiation and Polarization: New Molecules and Patterns of Gene Expression. J Immunol 177:7303-7311. doi:10.4049/jimmunol.177.10.7303.

30. Jablonski KA, Amici SA, Webb LM, Ruiz-Rosado J de D, Popovich PG, Partida-Sanchez S, Gueraude-Arellano M (2015) Novel Markers to Delineate Murine M1 and M2 Macrophages. PLoS One 10:e0145342.

31. Zhang J, Liu W-L, Tang DC, Chen L, Wang M, Pack SD, Zhuang Z, Rodgers GP (2002) Identification and characterization of a novel member of olfactomedin-related protein family, hGC-1, expressed during myeloid lineage development. Gene 283:83-93. doi:https://doi. org/10.1016/S0378-1119(01)00763-6.

32. Clemmensen SN, Bohr CT, Rørvig S, Glenthøj A, Mora-Jensen H, Cramer EP, Jacobsen LC, Larsen MT, Cowland JB, Tanassi JT, Heegaard NHH, Wren JD, Silahtaroglu AN, Borregaard N (2012) Olfactomedin 4 defines a subset of human neutrophils. J Leukoc Biol 91:495-500. doi:10.1189/ jlb.0811417.

33. Alder MN, Mallela J, Opoka AM, Lahni P, Hildeman DA, Wong HR (2018) Olfactomedin 4 marks a subset of neutrophils in mice. Innate Immun 25:22-33. doi:10.1177/1753425918817611.

34. Lahiri A, Hedl M, Yan J, Abraham C (2017) Human LACC1 increases innate receptor-induced responses and a LACC1 disease-risk variant modulates these outcomes. Nat Commun 8:15614.

35. Nathan CF, Hibbs JB (1991) Role of nitric oxide synthesis in macrophage antimicrobial activity. Curr Opin Immunol 3:65-70. doi:https://doi.org/10.1016/0952-7915(91)90079-G.

36. Sekelova Z, Stepanova H, Polansky O, Varmuzova K, Faldynova M, Fedr R, Rychlik I, Vlasatikova L (2017) Differential protein expression in chicken macrophages and heterophils in vivo following infection with Salmonella Enteritidis. Vet Res 48:35. doi:10.1186/s13567-017-0439-0.

37. Beyer M, Mallmann MR, Xue J, Staratschek-jox A, Vorholt D, Krebs W, Sommer D, Sander J, Mertens C, Nino-castro A, Schmidt S V, Schultze JL (2012) High-Resolution Transcriptome of Human Macrophages. PLoS One 7: doi:10.1371/journal.pone.0045466.

38. Carmans S, Hendriks JJA, Thewissen K, Van den Eynden J, Stinissen P, Rigo J-M, Hellings N (2010) The inhibitory neurotransmitter glycine modulates macrophage activity by activation of neutral amino acid transporters. J Neurosci Res 88:2420-2430. doi:10.1002/jnr.22395.

39. Loomis WP, den Hartigh AB, Cookson BT, Fink SL (2019) Diverse small molecules prevent macrophage lysis during pyroptosis. Cell Death Dis 10:326. doi:10.1038/s41419-019-1559-4.

40. Van den Bossche J, O’Neill LA, Menon D (2017) Macrophage Immunometabolism: Where Are We (Going)? Trends Immunol 38:395-406. doi:10.1016/j.it.2017.03.001. 
41. O'Neill LAJ, Artyomov MN (2019) Itaconate: the poster child of metabolic reprogramming in macrophage function. Nat Rev Immunol 19:273-281. doi:10.1038/s41577-019-0128-5.

42. Li D, Chen XQ, Li W-J, Yang Y-H, Wang J-Z, Yu ACH (2007) Cytoglobin Up-regulated by Hydrogen Peroxide Plays a Protective Role in Oxidative Stress. Neurochem Res 32:1375-1380. doi:10.1007/ s11064-007-9317-x.

43. Oleksiewicz U, Liloglou T, Field JK, Xinarianos G (2011) Cytoglobin: biochemical, functional and clinical perspective of the newest member of the globin family. Cell Mol Life Sci 68:38693883. doi:10.1007/s00018-011-0764-9.

44. Abdelkhalek NK, Komiya A, Kato-Unoki Y, Somamoto T, Nakao M (2009) Molecular evidence for the existence of two distinct IL-8 lineages of teleost CXC-chemokines. Fish Shellish Immunol 27:763-767. doi:10.1016/j.fsi.2009.08.004.

45. Deng Q, Sarris M, Bennin DA, Green JM, Herbomel P, Huttenlocher A (2013) Localized bacterial infection induces systemic activation of neutrophils through Cxcr2 signaling in zebrafish. $J$ Leukoc Biol 93:761-769. doi:10.1189/jlb.1012534.

46. de Oliveira S, Lopez-Muñoz A, Martínez-Navarro FJ, Galindo-Villegas J, Mulero V, Calado $\hat{A}$ (2015) Cxcl8-11 and Cxcl8-12 are required in the zebrafish defense against Salmonella Typhimurium. Dev Comp Immunol 49:44-48. doi:10.1016/j.dci.2014.11.004.

47. Fox SB, Moghaddam A, Westwood M, Turley H, Bicknell R, Gatter KC, Harris AL (1995) Plateletderived endothelial cell growth factor/thymidine phosphorylase expression in normal tissues: An immunohistochemical study. J Pathol 176:183-190. doi:10.1002/path.1711760212.

48. Walsh MC, Choi Y (2014) Biology of the RANKL-RANK-OPG System in Immunity, Bone, and Beyond. Front Immunol 5:511. doi:10.3389/fimmu.2014.00511.

49. Ahmed SBM, Prigent SA (2017) Insights into the Shc Family of Adaptor Proteins. J Mol Signal 12:2. doi:10.5334/1750-2187-12-2.

50. Chen C-C, Mo F-E, Lau LF (2001) The Angiogenic Factor Cyr61 Activates a Genetic Program for Wound Healing in Human Skin Fibroblasts. J Biol Chem 276:47329-47337. doi:10.1074/jbc. M107666200.

51. Chen C-C, Lau LF (2009) Functions and mechanisms of action of CCN matricellular proteins. Int J Biochem Cell Biol 41:771-783. doi:https://doi.org/10.1016/j.biocel.2008.07.025.

52. Orecchioni M, Ghosheh Y, Pramod AB, Ley K (2019) Macrophage Polarization: Different Gene Signatures in M1(LPS+) vs. Classically and M2(LPS-) vs. Alternatively Activated Macrophages. Front Immunol 10:1-14. doi:10.3389/fimmu.2019.01084.

53. Martinez FO, Gordon S (2014) The M1 and M2 paradigm of macrophage activation : time for reassessment. F1000 Prime Reports 6-13. doi:10.12703/P6-13.

54. Nadella V, Wang Z, Johnson TS, Griffin M, Devitt A (2015) Transglutaminase 2 interacts with syndecan-4 and CD44 at the surface of human macrophages to promote removal of apoptotic cells. Biochim Biophys Acta - Mol Cell Res. https://doi.org/10.1016/j.bbamcr.2014.09.020.

55. Sun H, Kaartinen MT (2018) Transglutaminases in Monocytes and Macrophages. Med Sci (Basel, Switzerland) 6:115. doi:10.3390/medsci6040115. 
56. McLatchie LM, Fraser NJ, Main MJ, Wise A, Brown J, Thompson N, Solari R, Lee MG, Foord SM (1998) RAMPs regulate the transport and ligand specificity of the calcitonin-receptor-like receptor. Nature 393:333-339. doi:10.1038/30666.

57. Chen P, Huang Y, Bong R, Ding Y, Song N, Wang X, Song X, Luo Y (2011) Tumor-Associated Macrophages Promote Angiogenesis and Melanoma Growth via Adrenomedullin in a Paracrine and Autocrine Manner. Clin Cancer Res 17:7230 LP - 7239. doi:10.1158/1078-0432.CCR-111354.

58. Pang X, Shang H, Deng B, Wen F, Zhang Y (2013) The Interaction of Adrenomedullin and Macrophages Induces Ovarian Cancer Cell Migration via Activation of RhoA Signaling Pathway. Int J Mol Sci 14:2774-2787. doi:10.3390/ijms14022774.

59. Rogers C, Fernandes-Alnemri T, Mayes L, Alnemri D, Cingolani G, Alnemri ES (2017) Cleavage of DFNA 5 by caspase- 3 during apoptosis mediates progression to secondary necrotic/pyroptotic cell death. Nat Commun 8:14128. doi:10.1038/ncomms14128.

60. Chen KW, Demarco B, Heilig R, Shkarina K, Boettcher A, Farady CJ, Pelczar P, Broz P (2019) Extrinsic and intrinsic apoptosis activate pannexin-1 to drive NLRP3 inflammasome assembly. ЕМВО J 38:e101638. doi:10.15252/embj.2019101638.

61. Broz P, Pelegrín P, Shao F (2019) The gasdermins, a protein family executing cell death and inflammation. Nat Rev Immunol. doi:10.1038/s41577-019-0228-2.

62. Munder M, Eichmann K, Morán JM, Centeno F, Soler G, Modolell M (1999) Th1/Th2-Regulated Expression of Arginase Isoforms in Murine Macrophages and Dendritic Cells. I Immunol 163:3771 LP - 3777.

63. Jin S-LC, Lan L, Zoudilova M, Conti M (2005) Specific Role of Phosphodiesterase 4B in Lipopolysaccharide-Induced Signaling in Mouse Macrophages. J Immunol 175:1523 LP - 1531. doi:10.4049/jimmunol.175.3.1523.

64. Hertz AL, Bender AT, Smith KC, Gilchrist M, Amieux PS, Aderem A, Beavo JA (2009) Elevated cyclic AMP and PDE4 inhibition induce chemokine expression in human monocyte-derived macrophages. Proc Natl Acad Sci 106:21978 LP - 21983. doi:10.1073/pnas.0911684106.

65. Yang J-X, Hsieh K-C, Chen Y-L, Lee C-K, Conti M, Chuang T-H, Wu C-P, Jin S-LC (2017) Phosphodiesterase 4B negatively regulates endotoxin-activated interleukin-1 receptor antagonist responses in macrophages. Sci Rep 7:46165. doi:10.1038/srep46165.

66. Stockmann C, Kirmse S, Helfrich I, Weidemann A, Takeda N, Doedens A, Johnson RS (2011) A Wound Size Dependent Effect of Myeloid Cell Derived Vascular Endothelial Growth Factor on Wound Healing. J Invest Dermatol 131:797-801. doi:10.1038/jid.2010.345.

67. Rőszer T (2015) Understanding the Mysterious M2 Macrophage through Activation Markers and Effector Mechanisms. Mediators Inflamm 2015:1-16. doi:10.1155/2015/816460.

68. Espina J, Feijóo CG, Solís C, Glavic A (2013) csrnp1a Is Necessary for the Development of Primitive Hematopoiesis Progenitors in Zebrafish. PLoS One 8:e53858. doi: 10.1371/journal. pone.0053858.

69. Das A, Yang C-S, Arifuzzaman S, Kim S, Kim SY, Jung KH, Lee YS, Chai YG (2018) High-Resolution Mapping and Dynamics of the Transcriptome, Transcription Factors, and Transcription CoFactor Networks in Classically and Alternatively Activated Macrophages. Front Immunol 9:22. doi: 10.3389/fimmu.2018.00022. 
70. Eichelbaum K, Krijgsveld J (2014) Rapid Temporal Dynamics of Transcription, Protein Synthesis, and Secretion during Macrophage Activation. Mol \&amp;amp; Cell Proteomics 13:792 LP - 810. doi:10.1074/mcp.M113.030916.

71. Shirakata Y, Kimura R, Nanba D, Iwamoto R, Tokumaru S, Morimoto C, Yokota K, Nakamura M, Sayama K, Mekada E, Higashiyama S, Hashimoto K (2005) Heparin-binding EGF-like growth factor accelerates keratinocyte migration and skin wound healing. J Cell Sci 118:2363 LP - 2370. doi:10.1242/jcs.02346.

72. Edwards JP, Zhang X, Mosser DM (2009) The Expression of Heparin-Binding Epidermal Growth Factor-Like Growth Factor by Regulatory Macrophages. J Immunol 182:1929 LP - 1939. doi:10.4049/jimmunol.0802703.

73. Oteng A-B, Ruppert PMM, Boutens L, Dijk W, van Dierendonck XAMH, Olivecrona G, Stienstra R, Kersten S (2019) Characterization of ANGPTL4 function in macrophages and adipocytes using Angptl4-knockout and Angptl4-hypomorphic mice. J Lipid Res 60:1741-1754.

74. Feingold KR, Shigenaga JK, Cross AS, Moser A, Grunfeld C (2012) Angiopoietin like protein 4 expression is decreased in activated macrophages. Biochem Biophys Res Commun 421:612-615. doi:https://doi.org/10.1016/j.bbrc.2012.04.055.

75. Cho DI, Kang H-J, Jeon JH, Eom GH, Cho HH, Kim MR, Cho M, Jeong H-Y, Cho HC, Hong MH, Kim YS, Ahn Y (2019) Antiinflammatory activity of ANGPTL4 facilitates macrophage polarization to induce cardiac repair. JCI insight 4:e125437. doi:10.1172/jci.insight.125437.

76. Wary KK, Humtsoe JO (2005) Anti-lipid phosphate phosphohydrolase-3 (LPP3) antibody inhibits bFGF- and VEGF-induced capillary morphogenesis of endothelial cells. Cell Commun Signal 3:9. doi:10.1186/1478-811X-3-9.

77. Gustafsson C, Mjösberg J, Matussek A, Geffers R, Matthiesen L, Berg G, Sharma S, Buer J, Ernerudh J (2008) Gene Expression Profiling of Human Decidual Macrophages: Evidence for Immunosuppressive Phenotype. PLoS One 3:e2078.

78. Panchatcharam M, Salous AK, Brandon J, Miriyala S, Wheeler J, Patil P, Sunkara M, Morris AJ, Escalante-Alcalde D, Smyth SS (2014) Mice with targeted inactivation of ppap2b in endothelial and hematopoietic cells display enhanced vascular inflammation and permeability. Arterioscler Thromb Vasc Biol 34:837-845. doi:10.1161/ATVBAHA.113.302335.

79. Della Fazia MA, Servillo G, Sassone-Corsi P (1997) Cyclic AMP signalling and cellular proliferation: regulation of CREB and CREM. FEBS Lett 410:22-24. doi:10.1016/S00145793(97)00445-6.

80. Mueller TD, Zhang J, Sebald W, Duschl A (2002) Structure, binding , and antagonists in the IL-4 / IL-13 receptor system. 1592:237-250.

81. Lu X-J, Chen J (2019) Specific function and modulation of teleost monocytes/macrophages: polarization and phagocytosis. Zool Res 40:146-150. doi:10.24272/j.issn.2095-8137.2019.035.

82. Nathan BYCF, Murray HW, Wlebe IE, Rubin BY (1983) Berish y. 158:670-689.

83. Stein M, Keshav S, Harris N, Gordon S (1992) Interleukin 4 potently enhances murine macrophage mannose receptor activity: a marker of alternative immunologic macrophage activation. J Exp Med 176:287-92. 
84. Fleetwood AJ, Lawrence T, Hamilton JA, Cook AD (2007) Granulocyte-macrophage colonystimulating factor (CSF) and macrophage CSF-dependent macrophage phenotypes display differences in cytokine profiles and transcription factor activities: implications for CSF blockade in inflammation. J Immunol 178:5245-52. doi:10.4049/jimmunol.178.8.5245.

85. Secombes CJ, Zou J (2017) Evolution of Interferons and Interferon Receptors. Front Immunol 8:209. 10.3389/fimmu.2017.00209.

86. Wang T, Secombes CJ (2015) The evolution of IL-4 and IL-13 and their receptor subunits. Cytokine 75:8-13. doi:10.1016/j.cyto.2015.04.012.

87. Pereiro P, Figueras A, Novoa B (2019) Insights into teleost interferon-gamma biology: An update. Fish Shellfish Immunol 90:150-164. doi:10.1016/j.fsi.2019.04.002.

88. Yamaguchi T, Miyata S, Katakura F, Nagasawa T, Shibasaki Y, Yabu T, Fischer U, Nakayasu C, Nakanishi T, Moritomo T (2016) Recombinant carp IL-4/13B stimulates in vitro proliferation of carp IgM+ B cells. Fish Shellfish Immunol 49:225-229. doi:10.1016/J.FSI.2015.12.043.

89. Zhu L, Pan P, Fang W, Shao J, Xiang L (2012) Essential Role of IL-4 and IL-4R $\alpha$ Interaction in Adaptive Immunity of Zebrafish: Insight into the Origin of Th2-like Regulatory Mechanism in Ancient Vertebrates. J Immunol 188:5571 LP - 5584. doi:10.4049/jimmunol.1102259.

90. Wang T, Hanington PC, Belosevic M, Secombes CJ (2008) Two Macrophage Colony-Stimulating Factor Genes Exist in Fish That Differ in Gene Organization and Are Differentially Expressed. J Immunol 181:3310 LP - 3322. doi:10.4049/jimmunol.181.5.3310.

91. Yang K, Feng S, Zhang S, Yin L, Zhou H, Zhang A, Wang X (2019) Characterization of a new il-4/13 homologue in grass carp (Ctenopharyngodon idella) and its cooperation with M-CSF to promote macrophage proliferation. Fish Shellfish Immunol 93:508-516. doi:10.1016/j. fsi.2019.07.070.

92. Dijkstra JM (2014) TH 2 and Treg candidate genes in elephant shark. Nature 511:E7-E9. doi:10.1038/nature13446.

93. Yamaguchi T, Takizawa F, Fischer U, Dijkstra J (2015) Along the Axis between Type 1 and Type 2 Immunity; Principles Conserved in Evolution from Fish to Mammals. Biology (Basel) 4:814-859. doi:10.3390/biology4040814.

94. Petit J, David L, Dirks R, Wiegertjes GF (2017) Genomic and transcriptomic approaches to study immunology in cyprinids: What is next? Dev Comp Immunol 75:48-62. doi:10.1016/j. dci.2017.02.022.

95. KLEIN SD, BRÜNE B (2002) Heat-shock protein 70 attenuates nitric oxide-induced apoptosis in RAW macrophages by preventing cytochrome c release. Biochem J 362:635-641. doi:10.1042/ bj3620635.

96. Chong K-Y, Lai C-C, Lille S, Chang C, Su C-Y (1998) Stable Overexpression of the Constitutive Form of Heat Shock Protein 70 Confers Oxidative Protection. J Mol Cell Cardiol 30:599-608. doi:10.1006/jmcc.1997.0623.

97. Rao Y, Wan Q, Su H, Xiao X, Liao Z, Ji J, Yang C, Lin L, Su J (2018) ROS-induced HSP70 promotes cytoplasmic translocation of high-mobility group box $1 \mathrm{~b}$ and stimulates antiviral autophagy in grass carp kidney cells. J Biol Chem 293:17387-17401. doi:10.1074/jbc.RA118.003840. 
98. Martinez FO, Helming L, Milde R, Varin A, Melgert BN, Draijer C, Thomas B, Fabbri M, Crawshaw A, Ho LP, Ten Hacken NH, Cobos Jiménez V, Kootstra NA, Hamann J, Greaves DR, Locati M, Mantovani A, Gordon S (2013) Genetic programs expressed in resting and IL-4 alternatively activated mouse and human macrophages: similarities and differences. Blood 121:e57-e69. doi:10.1182/blood-2012-06-436212.

99. Meeker ND, Trede NS (2008) Immunology and zebrafish: Spawning new models of human disease. Dev Comp Immunol 32:745-757. doi:https://doi.org/10.1016/j.dci.2007.11.011.

100. Patton EE, Tobin DM (2019) Spotlight on zebrafish: the next wave of translational research. Dis Model amp amp Mech 12:dmm039370. doi:10.1242/dmm.039370.

101. Irnazarow I (1995) Genetic variability of Polish and Hungarian carp lines. Aquaculture 129:215. doi:10.1016/0044-8486(95)91961-t.

102. Saeij JPJ, Van Muiswinkel WB, Groeneveld A, Wiegertjes GF (2002) Immune modulation by fish kinetoplastid parasites: a role for nitric oxide. Parasitology 124:77-86. doi:10.1017/ S0031182001008915.

103. Kolder ICRM, van der Plas-Duivesteijn SJ, Tan G, Wiegertjes GF, Forlenza M, Guler AT, Travin DY, Nakao M, Moritomo T, Irnazarow I, den Dunnen JT, Anvar SY, Jansen HJ, Dirks RP, Palmblad M, Lenhard B, Henkel C V, Spaink HP (2016) A full-body transcriptome and proteome resource for the European common carp. BMC Genomics 17:701. doi:10.1186/s12864-016-3038-y.

104. Petit J, Bailey EC, Wheeler RT, de Oliveira CAF, Forlenza M, Wiegertjes GF (2019) Studies Into $\beta$-Glucan Recognition in Fish Suggests a Key Role for the C-Type Lectin Pathway. Front Immunol 10:280. doi: 10.3389/fimmu.2019.00280.

105. Li H, Handsaker B, Wysoker A, Fennell T, Ruan J, Homer N, Marth G, Abecasis G, Durbin R, Subgroup 1000 Genome Project Data Processing (2009) The Sequence Alignment/Map format and SAMtools. Bioinformatics 25:2078-2079. doi:10.1093/bioinformatics/btp352

106. Anders S, Pyl PT, Huber W (2014) HTSeq-a Python framework to work with high-throughput sequencing data. Bioinformatics 31:166-169. doi:10.1093/bioinformatics/btu638

107. Love MI, Huber W, Anders S (2014) Moderated estimation of fold change and dispersion for RNA-seq data with DESeq2. Genome Biol 15:550. doi:10.1186/s13059-014-0550-8

108. Chen H, Boutros PC (2011) VennDiagram: a package for the generation of highly-customizable Venn and Euler diagrams in R. BMC Bioinformatics 12:35. doi:10.1186/1471-2105-12-35

109. Forlenza M, Kaiser T, Savelkoul HFJ, Wiegertjes GF (2012) The use of real-time quantitative PCR for the analysis of cytokine mRNA levels. Methods Mol Biol 820:7-23. doi:10.1007/978-161779-439 


\section{Supplementary data: Transcriptome sequencing supports a striking conservation of macrophage polarization in fish}

A

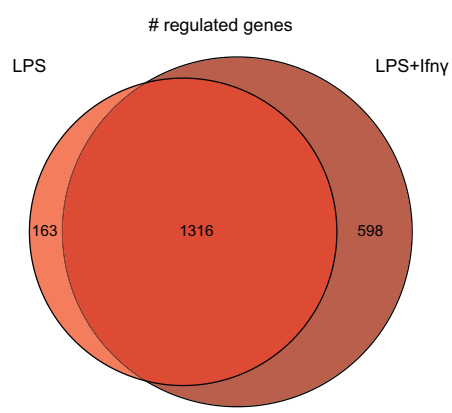

B

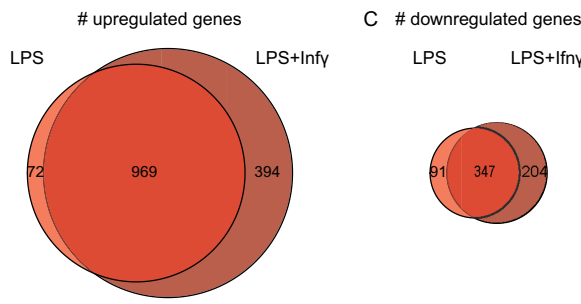

Supplementary figure 3.1 Addition of Ifn- $\gamma$ to LPS stimulation induces largely similar transcriptional profiles in carp macrophages. Proportional Venn diagrams depicting transcriptional changes of carp macrophages polarized for $6 \mathrm{~h}$ with $30 \mu \mathrm{g} / \mathrm{ml}$ LPS with (LPS + Ifn- $\gamma$ ) or without (LPS) the addition of 100 $\mathrm{ng} / \mathrm{ml}$ IFN- $\gamma$ compared to unpolarized control macrophages. The total number of significantly $\left(p_{\text {adiusted }}<0.05\right)$ regulated genes $(\mathbf{A})$ is further specified to show the number of genes upregulated (B) and downregulated (C). Data are of $n=3$ fish.
Supplementary figure 3.2 Bioactivity of recombinant Il-4/13b1 displayed as a downregulation of $\mathrm{T}$-borreli induced nitric oxide (NO) production. NO production of total mid-kidney leukocytes measured as nitrite concentration in culture supernatants after $96 \mathrm{~h}$. Cells were kept as unstimulated controls (grey), or stimulated with Il-4/13b1 (light blue), T. borreli lysate (T.bor, red) or T. borreli and Il-4/13b1 (dark blue). Data of $n=3$ fish were analyzed using a repeated measures ANOVA. Asterisks (*) indicate significant differences $p<0.05$. 
Supplementary table 3.1 Transcriptional phenotype of carp LPS + Ifn- $\gamma$ polarized macrophages is similar to LPS stimulated macrophages, with higher fold changes between stimulated and control cells. Genes most highly upregulated in M1 macrophages polarized with $30 \mu \mathrm{g} / \mathrm{ml} \mathrm{LPS}+100 \mathrm{ng} /$ $\mathrm{ml}$ Ifn- $\gamma$ for $6 \mathrm{~h}$ in descending order. Genes were included when the following criteria were met: $p_{\text {adjusted }}<$ 0.05 and average reads per kilobasepair per million reads (RPKM $>50)$ in stimulated or control samples. The 20 most highly upregulated genes (excluding paralogs) were depicted with the gene abbreviation (Gene), gene description, gene identifier (Gene ID cypCar), log2 fold change compared to unstimulated control macrophages (Log2FC), a short description of their main function (in macrophages if possible) and average RPKM in control (RPKM C) and LPS (RPKM LPS + Ifn- $\gamma$ ) polarized macrophages. Multiple cypCar IDs per gene were included if paralogs fell within the top 20 most upregulated genes. Each cypCar gene ID represents an individual gene unless cypCars are combined with a dash (-), indicating a (most likely) single gene that was wrongly predicted as two genes. Data are of $n=3$ fish.

\begin{tabular}{|c|c|c|c|c|c|c|}
\hline Gene & Gene description & Gene ID & $\begin{array}{l}\log 2 \\
\text { FC }\end{array}$ & Main Function & RPKM C & $\begin{array}{l}\text { RPKM } \\
\text { LPS+ } \\
\text { Ifn-y }\end{array}$ \\
\hline \multirow{2}{*}{ il12p35 } & \multirow{2}{*}{$\begin{array}{l}\text { Interleukin } 12 \\
\text { subunit alpha (p35) }\end{array}$} & 00024698- & 11.0 & \multirow{2}{*}{$\begin{array}{l}\text { P35 subunit of the pro-inflammatory cytokine II-12. Involved } \\
\text { in the activation of Th1 and NK cells [1]. }\end{array}$} & 0.1 & 261.4 \\
\hline & & 00024699 & 9.6 & & 0.4 & 263.7 \\
\hline \multirow{2}{*}{$i / 1 b$} & \multirow{2}{*}{ Interleukin 1 beta } & 00043439- & 8.4 & \multirow{2}{*}{$\begin{array}{l}\text { Pro-inflammatory cytokine. Mediator of various cellular } \\
\text { activities including proliferation, differentiation and apoptosis } \\
\text { [2]. }\end{array}$} & 72.0 & 21098.7 \\
\hline & & 00043440 & 8.2 & & 74.0 & 18253.6 \\
\hline agrn & Agrin & 00029572 & 7.3 & $\begin{array}{l}\text { Extracellular-matrix protein involved in monocyte/ } \\
\text { macrophage survival, cytoskeleton formation and } \\
\text { phagocytosis [3]. }\end{array}$ & 32.7 & 2946.9 \\
\hline steap4 & $\begin{array}{l}\text { Six-transmembrane } \\
\text { epithelial antigen of } \\
\text { prostate } 4\end{array}$ & 00042005 & 7.1 & $\begin{array}{l}\text { Metalloreductase involved in the transfer of ions from Fe3+ } \\
\text { and } \mathrm{Cu} 2+\text { to NAD and plays a role in cellular homeostasis } \\
\text { during inflammation. Increased steap4 may reduce } \\
\text { circulating iron available for parasites [4]. }\end{array}$ & 4.6 & 384.7 \\
\hline \multirow{2}{*}{ saa } & \multirow{2}{*}{$\begin{array}{l}\text { Serum amyloid } A \\
\text { protein }\end{array}$} & 00037333 & 6.9 & \multirow{2}{*}{$\begin{array}{l}\text { Acute phase protein, chemotactic to phagocytes and induces } \\
\text { transcription of several pro-inflammatory cytokines }[5,6] \text {. }\end{array}$} & 2.8 & 270.3 \\
\hline & & 00036204 & 5.6 & & 77.6 & 2427.9 \\
\hline lacc1 & $\begin{array}{l}\text { Laccase-domain } \\
\text { containing protein } 1\end{array}$ & 00009189 & 6.2 & $\begin{array}{l}\text { Promotes fatty-acid oxidation, inflammasome activation, } \\
\text { mitochondrial and NADPH-oxidase-dependent reactive } \\
\text { oxygen species production and bactericidal activity of } \\
\text { macrophages [7]. }\end{array}$ & 4.3 & 232.9 \\
\hline \multirow{2}{*}{$\operatorname{nos} 2 b$} & \multicolumn{2}{|c|}{ Nitric oxide synthase 00004424} & 5.9 & \multirow{2}{*}{$\begin{array}{l}\text { Production of antimicrobial nitric oxide. Has functioned as } \\
\text { M2 marker since macrophage polarization was described } \\
{[8,9] \text {. }}\end{array}$} & 10.1 & 533.3 \\
\hline & $2 \mathrm{~b}$ & 00024539 & 5.9 & & 43.6 & 2183.6 \\
\hline $\begin{array}{l}\text { ptgs } 2 a / \\
\operatorname{cox} 2\end{array}$ & $\begin{array}{l}\text { Prostaglandin- } \\
\text { endoperoxide } \\
\text { synthase } 2 \mathrm{a}\end{array}$ & 00026925 & 5.8 & $\begin{array}{l}\text { Also known as Cox-2. Increased expression in human M1 } \\
\text { macrophages }[10,11] \text {. }\end{array}$ & 8.7 & 351.3 \\
\hline$m h c 2 d b b$ & $\begin{array}{l}\text { Major } \\
\text { histocompatibility } \\
\text { complex class II } \\
\text { DBB }\end{array}$ & 00014759 & 5.5 & $\begin{array}{l}\text { Classified as non-classical MHC II with conserved domains } \\
\text { for CD4 binding }[12,13] \text {. No functional studies have been } \\
\text { performed. }\end{array}$ & 1.7 & 64.7 \\
\hline$z g c: 174917$ & $\begin{array}{l}7 \text { Uncharacterized } \\
\text { protein }\end{array}$ & 00021523 & 5.4 & & 1.9 & 68.1 \\
\hline $\begin{array}{l}\text { mpeg1.2/ } \\
\text { perforin } 2\end{array}$ & $\begin{array}{l}\text { Macrophage- } \\
\text { expressed } 1 / \\
\text { Perforin } 2\end{array}$ & 00002880 & 5.2 & $\begin{array}{l}\text { Pore forming perforin. Induced upon infection in zebrafish } \\
\text { macrophages [14]. }\end{array}$ & 2.0 & 64.0 \\
\hline olfm4 & Olfactomedin-4-like & 00047183 & 5.1 & $\begin{array}{l}\text { Extracellular glycoprotein indicated in myeloid-specific } \\
\text { differentiation and neutrophil inflammation [15-17] }\end{array}$ & 1.8 & 51.7 \\
\hline tmem238 & $\begin{array}{l}\text { Transmembrane } \\
\text { protein } 238\end{array}$ & 00015661 & 5.0 & $\begin{array}{l}\text { Classified as transmembrane protein, no specific functions } \\
\text { have been described. }\end{array}$ & 2.4 & 59.1 \\
\hline \multirow[b]{2}{*}{ mecr } & \multirow{2}{*}{$\begin{array}{l}\text { Mitochondrial Enoyl- } \\
\text { [acyl-carrier-protein] } \\
\text { reductase }\end{array}$} & 00002503 & 5.0 & \multirow{2}{*}{$\begin{array}{l}\text { Protein involved in mitochondrial fatty acid synthesis. } \\
\text { Increased upon Salmonella enteritidis infection in chicken } \\
\text { macrophages [18]. }\end{array}$} & 13.6 & 302.0 \\
\hline & & 00002502 & 4.9 & & 24.9 & 568.4 \\
\hline il6 & Interleukin 6 & 00035927 & 5.0 & $\begin{array}{l}\text { Pro-inflammatory cytokine produced by macrophages in } \\
\text { response to PRR activation }[19,20] \text {. }\end{array}$ & 30.2 & 843.5 \\
\hline
\end{tabular}




\begin{tabular}{|c|c|c|c|c|c|c|}
\hline Gene & Gene description & Gene ID & $\begin{array}{l}\text { Log2 } \\
\text { FC }\end{array}$ & Main Function & RPKM C & $\begin{array}{l}\text { RPKM } \\
\text { LPS+ } \\
\text { Ifn-y }\end{array}$ \\
\hline $\begin{array}{l}\operatorname{acod} 1 / \\
\operatorname{irg} 1\end{array}$ & $\begin{array}{l}\text { Aconitate } \\
\text { decarboxylase 1/ } \\
\text { Immune responsive } \\
\text { gene } 1\end{array}$ & $\begin{array}{l}00007903 \\
00026281\end{array}$ & $\begin{array}{l}4.9 \\
4.9\end{array}$ & $\begin{array}{l}\text { Catalyzes the production of itaconate. High expression } \\
\text { in mammalian } \mathrm{M} 1 \text { macrophages contributes to metabolic } \\
\text { reprogramming }[21,22] \text {. }\end{array}$ & $\begin{array}{c}71.5 \\
6.4\end{array}$ & $\begin{array}{c}1752.0 \\
155.5\end{array}$ \\
\hline$t d h$ & $\begin{array}{l}\text { L-threonine } \\
\text { dehydrogenase }\end{array}$ & 00008269 & 4.7 & $\begin{array}{l}\text { Converts L-theonine into glycine. Glycine modulates } \\
\text { macrophage activity }[23,24] \text {. }\end{array}$ & 16.3 & 343.5 \\
\hline $\mid i 441$ & IL4 induced 1 & $\begin{array}{l}00008491 \\
00008492\end{array}$ & $\begin{array}{l}4.5 \\
4.4\end{array}$ & $\begin{array}{l}\text { Expressed by macrophages stimulated by microbial } \\
\text { derived products. Exerts antibacterial activities.through the } \\
\text { production of } \mathrm{H} 2 \mathrm{O} 2 \text { and other toxic metabolites [25]. }\end{array}$ & $\begin{array}{c}10.6 \\
9.3\end{array}$ & $\begin{array}{l}210.0 \\
162.4\end{array}$ \\
\hline $\begin{array}{l}\text { si:ch1073- } \\
67 j 19.1\end{array}$ & $\begin{array}{l}\text { Uncharacterized } \\
\text { protein }\end{array}$ & 00039673 & 4.5 & & 314.3 & 6004.4 \\
\hline cygb1 & Cytoglobin 1 & 00046202 & 4.3 & $\begin{array}{l}\text { Oxygen-carrying globin, expressed in macrophages and } \\
\text { increased during oxidative stress. Protection mechanism } \\
\text { against oxidative stress }[26,27] \text {. }\end{array}$ & 3.0 & 55.3 \\
\hline
\end{tabular}

1. Tait Wojno ED, Hunter CA, Stumhofer JS (2019) The Immunobiology of the Interleukin-12 Family: Room for Discovery. Immunity 50:851-870. doi:10.1016/j.immuni.2019.03.011.

2. Mantovani A, Dinarello CA, Molgora M, Garlanda C (2019) Interleukin-1 and Related Cytokines in the Regulation of Inflammation and Immunity. Immunity 50:778-795. doi:10.1016/j. immuni.2019.03.012.

3. Mazzon C, Anselmo A, Soldani C, Cibella J, Ploia C, Moalli F, Burden SJ, Dustin ML, Sarukhan A, Viola A (2012) Agrin is required for survival and function of monocytic cells. Blood 119:55025511. doi:10.1182/blood-2011-09-382812.

4. Scarl RT, Lawrence CM, Gordon HM, Nunemaker CS (2017) STEAP4: its emerging role in metabolism and homeostasis of cellular iron and copper. $J$ Endocrinol 234:R123-R134. doi:10.1530/JOE-16-0594.

5. Badolato R, Wang JM, Murphy WJ, Lloyd AR, Michiel DF, Bausserman LL, Kelvin DJ, Oppenheim IJ (1994) Serum amyloid A is a chemoattractant: induction of migration, adhesion, and tissue infiltration of monocytes and polymorphonuclear leukocytes. J Exp Med 180:203-209. doi:10.1084/jem.180.1.203.

6. He RL, Zhou J, Hanson CZ, Chen J, Cheng N, Ye RD (2009) Serum amyloid A induces G-CSF expression and neutrophilia via Toll-like receptor 2. Blood 113:429-437. doi:10.1182/ blood-2008-03-139923.

7. Lahiri A, Hedl M, Yan J, Abraham C (2017) Human LACC1 increases innate receptor-induced responses and a LACC1 disease-risk variant modulates these outcomes. Nat Commun 8:15614.

8. Nathan CF, Hibbs JB (1991) Role of nitric oxide synthesis in macrophage antimicrobial activity. Curr Opin Immunol 3:65-70. doi:10.1016/0952-7915(91)90079-G.

9. Mills CD, Kincaid K, Alt JM, Heilman MJ, Hill AM (2000) M-1/M-2 Macrophages and the Th1/ Th2 Paradigm. J Immunol 164:6166-6173. doi:10.4049/jimmunol.164.12.6166.

10. Martinez FO, Gordon S, Locati M, Mantovani A (2006) Transcriptional Profiling of the Human Monocyte-to-Macrophage Differentiation and Polarization: New Molecules and Patterns of Gene Expression. J Immunol 177:7303-7311. doi:10.4049/jimmunol.177.10.7303.

11. Jablonski KA, Amici SA, Webb LM, Ruiz-Rosado J de D, Popovich PG, Partida-Sanchez S, Gueraude-Arellano M (2015) Novel Markers to Delineate Murine M1 and M2 Macrophages. PLoS One 10:e0145342. 
12. Sambrook JG, Figueroa F, Beck S (2005) A genome-wide survey of Major Histocompatibility Complex (MHC) genes and their paralogues in zebrafish. BMC Genomics 6:152. doi:10.1186/14712164-6-152.

13. Dijkstra JM, Grimholt U, Leong J, Koop BF, Hashimoto K (2013) Comprehensive analysis of MHC class II genes in teleost fish genomes reveals dispensability of the peptide-loading DM system in a large part of vertebrates. BMC Evol Biol 13:260. doi:10.1186/1471-2148-13-260.

14. Benard EL, Racz PI, Rougeot J, Nezhinsky AE, Verbeek FJ, Spaink HP, Meijer AH (2015) Macrophage-Expressed Perforins Mpeg1 and Mpeg1.2 Have an Anti-Bacterial Function in Zebrafish. J Innate Immun 7:136-152. doi:10.1159/000366103.

15. Zhang J, Liu W-L, Tang DC, Chen L, Wang M, Pack SD, Zhuang Z, Rodgers GP (2002) Identification and characterization of a novel member of olfactomedin-related protein family, hGC-1, expressed during myeloid lineage development. Gene 283:83-93. doi:10.1016/S03781119(01)00763-6.

16. Clemmensen SN, Bohr CT, Rørvig S, Glenthøj A, Mora-Jensen H, Cramer EP, Jacobsen LC, Larsen MT, Cowland JB, Tanassi JT, Heegaard NHH, Wren JD, Silahtaroglu AN, Borregaard N (2012) Olfactomedin 4 defines a subset of human neutrophils. J Leukoc Biol 91:495-500. doi:10.1189/ jlb.0811417.

17. Alder MN, Mallela J, Opoka AM, Lahni P, Hildeman DA, Wong HR (2018) Olfactomedin 4 marks a subset of neutrophils in mice. Innate Immun 25:22-33. doi:10.1177/1753425918817611.

18. Sekelova Z, Stepanova H, Polansky O, Varmuzova K, Faldynova M, Fedr R, Rychlik I, Vlasatikova L (2017) Differential protein expression in chicken macrophages and heterophils in vivo following infection with Salmonella Enteritidis. Vet Res 48:35. doi:10.1186/s13567-017-0439-0.

19. Mantovani A, Sica A, Sozzani S, Allavena P, Vecchi A, Locati M (2004) The chemokine system in diverse forms of macrophage activation and polarization. Trends Immunol 25:677-686. doi:10.1016/j.it.2004.09.015.

20. Beyer M, Mallmann MR, Xue J, Staratschek-jox A, Vorholt D, Krebs W, Sommer D, Sander J, Mertens C, Nino-castro A, Schmidt S V, Schultze JL (2012) High-Resolution Transcriptome of Human Macrophages. PLoS One 7:e45466. doi:10.1371/journal.pone.0045466.

21. Van den Bossche J, O’Neill LA, Menon D (2017) Macrophage Immunometabolism: Where Are We (Going)? Trends Immunol 38:395-406. doi:10.1016/j.it.2017.03.001.

22. O'Neill LAJ, Artyomov MN (2019) Itaconate: the poster child of metabolic reprogramming in macrophage function. Nat Rev Immunol 19:273-281. doi:10.1038/s41577-019-0128-5.

23. Carmans S, Hendriks JJA, Thewissen K, Van den Eynden J, Stinissen P, Rigo J-M, Hellings N (2010) The inhibitory neurotransmitter glycine modulates macrophage activity by activation of neutral amino acid transporters. J Neurosci Res 88:2420-2430. doi:10.1002/jnr.22395.

24. Loomis WP, den Hartigh AB, Cookson BT, Fink SL (2019) Diverse small molecules prevent macrophage lysis during pyroptosis. Cell Death Dis 10:326. doi:10.1038/s41419-019-1559-4.

25. Puiffe M-L, Lachaise I, Molinier-Frenkel V, Castellano F (2013) Antibacterial Properties of the Mammalian L-Amino Acid Oxidase IL4I1. PLoS One 8:e54589. 
26. Li D, Chen XQ, Li W-J, Yang Y-H, Wang J-Z, Yu ACH (2007) Cytoglobin Up-regulated by Hydrogen Peroxide Plays a Protective Role in Oxidative Stress. Neurochem Res 32:1375-1380. doi:10.1007/ s11064-007-9317-x.

27. Oleksiewicz U, Liloglou T, Field JK, Xinarianos G (2011) Cytoglobin: biochemical, functional and clinical perspective of the newest member of the globin family. Cell Mol Life Sci 68:38693883. doi:10.1007/s00018-011-0764-9.

\begin{tabular}{|lcc|}
\hline Gene & $\begin{array}{c}\text { Gene ID } \\
\text { cypCar }\end{array}$ & $\begin{array}{c}\text { Average } \\
\text { RPKM C }\end{array}$ \\
\hline il4r2 & 00012277 & 30 \\
\hline \multirow{2}{*}{ il13ra1 } & 00023082 & 163 \\
& 00003131 & 29 \\
\hline \multirow{2}{*}{ il13ra2 } & 00000342 & 30 \\
& 00011497 & 166 \\
\hline \multirow{2}{*}{ il2rga } & 00020372 & 583 \\
& 00009989 & 151 \\
\hline il2rgb & 00003404 & 55 \\
\multirow{2}{*}{ stat6 } & 00003253 & 20 \\
& 00024537 & 28 \\
\hline \multirow{2}{*}{ stat3 } & 00029051 & 95 \\
& 00030463 & 64 \\
& 00030464 & 33 \\
\hline
\end{tabular}

Supplementary table 3.2 Putative receptors and downstream signaling molecules of $\mathrm{Il}-4 / 13$ are expressed in unstimulated macrophages. Putative receptor subunits and downstream signaling molecules were depicted with the gene abbreviation (Gene), gene identifier (Gene ID cypCar) and average RPKM in unstimulated control macrophages (RPKM C) of $n=3$ fish.

Supplementary table 3.3 Marker candidates for M1 and/or M2. Candidate markers meeting the following requirements for M1 and/or M2 macrophages (indicated in bold); significantly ( $p_{\text {adjusted }}<$ 0.05) up-or downregulation compared to unstimulated controls $>1.5 \log 2$ or $<-1.5 \log 2$, difference in regulation between M1 and M2 > $2.5 \log 2$ and average RPKM > 50 in the stimulated samples or unstimulated controls. Candidate markers depicted with the gene identifier (Gene ID cypCar), the gene abbreviation (Gene), log2 fold change (Log2FC) in M1 and M2 macrophages compared to unstimulated controls and average RPKM in M1 and M2 macrophages and corresponding unstimulated control (C) macrophages of $n=3$ fish. NA indicates an absence of log2FC calculations due to expression below the detection limit in one or multiple samples. Letters in superscript next to the gene abbreviation indicate a possible mis-annotation of a single gene as two separate genes.

\begin{tabular}{|c|c|c|c|c|c|c|c|}
\hline $\begin{array}{l}\text { Gene ID } \\
\text { cypCar }\end{array}$ & Gene & Log2FC M1 & RPKM C & RPKM M1 & Log2FC M2 & $\begin{array}{c}\text { RPKM C } \\
\text { M2 }\end{array}$ & RPKM M2 \\
\hline \multicolumn{8}{|c|}{ Marker candidates for M1 and M2 macrophages } \\
\hline \multicolumn{8}{|c|}{ Significantly upregulated in M1 and significantly downregulated in M2 } \\
\hline 00046689 & marcks/1a & 1.7 & 155.2 & 432.1 & -1.6 & 204.1 & 54.8 \\
\hline \multicolumn{8}{|c|}{ Significantly downregulated in M1 and significantly upregulated in M2 } \\
\hline 00047225 & $m r c 1 b$ & -1.7 & 217.8 & 66.1 & 2.0 & 125.8 & 432.0 \\
\hline 00023855 & $m r c 1 b$ & -1.6 & 192.2 & 60.6 & 2.0 & 119.1 & 393.9 \\
\hline 00046879 & $m r c 1 b$ & -1.5 & 1188.3 & 369.0 & 1.7 & 615.0 & 1662.5 \\
\hline 00034006 & $m r c 1 b$ & -1.5 & 1053.0 & 336.0 & 1.7 & 543.6 & 1454.6 \\
\hline 00046880 & $m r c 1 b$ & -1.5 & 1590.7 & 527.2 & 1.6 & 857.0 & 2248.6 \\
\hline
\end{tabular}




\begin{tabular}{|c|c|c|c|c|c|c|c|}
\hline $\begin{array}{l}\text { Gene ID } \\
\text { cypCar }\end{array}$ & Gene & Log2FC M1 & RPKM C & RPKM M1 & Log2FC M2 & $\begin{array}{c}\text { RPKM C } \\
\text { M2 }\end{array}$ & RPKM M2 \\
\hline \multicolumn{8}{|c|}{ Marker candidates for M1 macrophages alone } \\
\hline \multicolumn{8}{|c|}{ Significantly upregulated in M1 and significantly downregulated in M2 } \\
\hline 00004424 & $n o s 2 b$ & 5.3 & 10.1 & 355.4 & -0.7 & 25.5 & 13.3 \\
\hline 00012414 & hsp70 & 3.2 & 55.0 & 479.5 & -0.9 & 41.3 & 16.9 \\
\hline 00012415 & $h s p 70^{a}$ & 3.2 & 43.5 & 393.8 & -0.9 & 34.9 & 14.2 \\
\hline 00000280 & hsp70 & 3.1 & 21.7 & 188.8 & -0.8 & 15.3 & 6.9 \\
\hline 00030534 & hsp70 & 3.1 & 50.5 & 397.1 & -0.8 & 36.1 & 15.9 \\
\hline 00048796 & hsp70 & 3.0 & 27.1 & 221.0 & -0.8 & 20.3 & 9.8 \\
\hline 00041000 & $c f b / c 2-a 3$ & 2.3 & 16.4 & 71.1 & -0.6 & 23.6 & 12.4 \\
\hline 00005739 & $c f b / c 2-a 3$ & 2.3 & 240.3 & 1002.5 & -0.5 & 329.3 & 189.6 \\
\hline 00032673 & dnajb1b & 2.1 & 18.5 & 70.3 & -1.4 & 14.5 & 4.7 \\
\hline 00045205 & $f 3 b$ & 2.0 & 72.9 & 229.3 & -0.8 & 62.1 & 29.4 \\
\hline 00033031 & malt3 & 1.9 & 23.8 & 80.8 & -1.1 & 39.5 & 17.0 \\
\hline \multicolumn{8}{|c|}{ Significantly upregulated exclusively in M1 } \\
\hline 00024698 & il12p35 & 8.8 & 0.1 & 56.0 & NA & 0.18 & 1.53 \\
\hline 00024699 & il12p35 & 7.2 & 0.4 & 59.4 & NA & 0.27 & 1.85 \\
\hline 00037333 & saa & 6.3 & 2.8 & 183.8 & 0.7 & 4.1 & 5.8 \\
\hline 00024539 & $n o s 2 b$ & 5.2 & 43.6 & 1427.6 & -0.4 & 102.4 & 62.9 \\
\hline 00046202 & $c y g b 1$ & 4.3 & 3.0 & 56.4 & 0.3 & 21.9 & 20.2 \\
\hline 00002926 & $c x c / 13 c$ & 4.0 & 8.6 & 96.4 & 0.3 & 6.4 & 6.8 \\
\hline 00016657 & $c x c / 8 / 1$ & 4.0 & 230.4 & 2984.1 & 0.3 & 98.1 & 99.4 \\
\hline 00008491 & $i \mid 4 i 1$ & 3.5 & 10.6 & 106.3 & 0.2 & 6.1 & 6.2 \\
\hline 00033150 & $i l 1 r 2$ & 3.5 & 15.2 & 137.9 & 0.0 & 14.4 & 12.5 \\
\hline 00015036 & slc2a6 & 3.4 & 29.0 & 267.1 & 0.1 & 61.6 & 60.7 \\
\hline 00024970 & $i l 1 r 2$ & 3.4 & 70.1 & 626.2 & 0.2 & 63.6 & 62.4 \\
\hline 00018821 & ptges & 3.4 & 7.7 & 68.3 & 0.2 & 9.2 & 8.6 \\
\hline 00008492 & $i l 4 i 1$ & 3.4 & 9.3 & 85.3 & 0.1 & 5.1 & 4.6 \\
\hline 00017325 & igsf6 & 3.4 & 17.3 & 164.2 & 0.2 & 26.1 & 25.5 \\
\hline 00007848 & blvrb & 3.3 & 8.7 & 76.1 & 0.2 & 13.0 & 12.7 \\
\hline 00002648 & $\begin{array}{l}\text { uncharac- } \\
\text { terized prot. }\end{array}$ & 3.1 & 18.5 & 134.4 & 0.2 & 19.5 & 18.1 \\
\hline 00028558 & mettl17 & 3.1 & 11.4 & 82.8 & 0.2 & 7.8 & 7.6 \\
\hline 00028560 & $p f 1$ & 2.7 & 67.6 & 346.2 & -0.2 & 50.0 & 33.6 \\
\hline 00032122 & flot $1 b$ & 2.5 & 45.8 & 229.7 & -0.4 & 59.7 & 38.8 \\
\hline 00028528 & tnfsf13b & 2.4 & 39.8 & 181.4 & -0.5 & 33.4 & 20.4 \\
\hline 00040937 & pak1 & 2.1 & 13.9 & 50.1 & -0.6 & 22.9 & 13.5 \\
\hline \multicolumn{8}{|c|}{ Significantly upregulated in both $M 1$ and $M 2$ but at least $2.5 \log 2$ higher in $M 1$ compared to $M 2$} \\
\hline 00043439 & $i / 1 b^{c}$ & 7.6 & 72.0 & 12832.1 & 4.2 & 46.1 & 945.4 \\
\hline 00043440 & $i / 1 b^{c}$ & 7.5 & 74.0 & 11482.7 & 4.0 & 42.2 & 771.8 \\
\hline 00042005 & steap4 & 7.0 & 4.6 & 405.9 & 3.8 & 6.8 & 100.6 \\
\hline 00029572 & agrn & 7.0 & 32.7 & 2569.8 & 4.3 & 11.2 & 153.8 \\
\hline 00026925 & ptgs2a & 5.7 & 8.7 & 319.8 & 2.8 & 5.7 & 33.6 \\
\hline
\end{tabular}




\begin{tabular}{|c|c|c|c|c|c|c|c|}
\hline $\begin{array}{l}\text { Gene ID } \\
\text { cypCar }\end{array}$ & Gene & Log2FC M1 & RPKM C & RPKM M1 & Log2FC M2 & $\begin{array}{c}\text { RPKM C } \\
\text { M2 }\end{array}$ & RPKM M2 \\
\hline 00047183 & olfm4 & 5.4 & 1.8 & 69.5 & 1.7 & 8.2 & 23.5 \\
\hline 00036204 & saa & 5.3 & 77.6 & 1968.3 & 1.4 & 83.1 & 192.6 \\
\hline 00002503 & mercr & 5.1 & 13.6 & 340.7 & 0.9 & 16.4 & 24.8 \\
\hline 00002502 & mercr & 5.0 & 24.9 & 627.8 & 1.0 & 29.4 & 46.7 \\
\hline 00035927 & il6 & 5.0 & 30.2 & 864.5 & 1.7 & 32.3 & 80.6 \\
\hline 00007903 & $\operatorname{irg} 1$ & 4.6 & 71.5 & 1404.8 & 1.9 & 129.3 & 406.7 \\
\hline 00026281 & $\operatorname{irg} 1$ & 4.5 & 6.4 & 121.8 & 1.9 & 12.2 & 37.3 \\
\hline \multicolumn{8}{|c|}{ Downregulated exclusively in M1 } \\
\hline 00017566 & $d a b 2$ & -2.8 & 91.2 & 12.7 & 0.1 & 71.8 & 63.4 \\
\hline \multicolumn{8}{|c|}{ Significantly downregulated in M1 and significantly upregulated in M2 } \\
\hline 00002074 & $d a b 2$ & -1.9 & 123.1 & 31.3 & 0.6 & 96.0 & 125.6 \\
\hline 00008536 & $f g / 2$ & -1.9 & 468.9 & 107.3 & 0.8 & 365.6 & 529.5 \\
\hline 00028026 & ca9 or ca14 & -1.8 & 55.3 & 14.1 & 0.9 & 42.7 & 65.7 \\
\hline \multicolumn{8}{|c|}{ Marker genes for M2 macrophages alone } \\
\hline \multicolumn{8}{|c|}{ Significantly upregulated in M2 and significantly downregulated in M1 } \\
\hline 00031163 & tcima & -0.9 & 138.7 & 62.0 & 3.1 & 86.7 & 556.2 \\
\hline 00027636 & rnf182 & -0.6 & 147.3 & 78.8 & 2.9 & 25.2 & 143.1 \\
\hline 00001546 & glud1a & -0.3 & 57.8 & 39.9 & 2.7 & 66.1 & 364.9 \\
\hline 00016733 & tinagl1 & -1.5 & 7.0 & 2.3 & 2.6 & 22.0 & 113.1 \\
\hline 00019572 & $\begin{array}{l}\text { uncharac- } \\
\text { terized prot. }\end{array}$ & -0.5 & 28.4 & 16.7 & 2.5 & 32.4 & 142.2 \\
\hline 00012277 & il4r.2 & -0.5 & 73.6 & 42.8 & 2.5 & 63.3 & 298.5 \\
\hline 00000704 & entpd1 & -1.1 & 45.3 & 18.4 & 2.2 & 36.6 & 140.7 \\
\hline 00037861 & $m a f b b$ & -1.1 & 188.8 & 81.1 & 1.9 & 135.4 & 434.8 \\
\hline 00014545 & $p / d 3$ & -1.2 & 26.5 & 9.9 & 1.9 & 23.2 & 72.6 \\
\hline 00023082 & il13ra1 & -0.7 & 408.4 & 220.5 & 1.9 & 228.9 & 699.4 \\
\hline 00001348 & hip1 & -1.0 & 60.1 & 25.9 & 1.7 & 44.9 & 117.0 \\
\hline 00020333 & zfp36/1a & -1.3 & 33.9 & 12.0 & 1.6 & 32.9 & 82.9 \\
\hline \multicolumn{8}{|c|}{ Significantly upregulated exclusively in M2 } \\
\hline 00030755 & timp2b & 0.5 & 30.7 & 36.1 & 8.0 & 31.1 & 6476.0 \\
\hline 00034483 & $\operatorname{tgm} 2 b^{\mathrm{d}}$ & 0.5 & 5.9 & 7.2 & 7.4 & 6.0 & 784.6 \\
\hline 00030329 & $\operatorname{tgm} 2 b^{d}$ & 0.8 & 4.8 & 6.9 & 6.9 & 6.4 & 698.7 \\
\hline 00041907 & $\operatorname{tgm} 2 b$ & -0.2 & 1.1 & 0.9 & 5.0 & 1.7 & 68.2 \\
\hline 00024882 & $p d e 4 b b$ & 0.4 & 22.5 & 23.7 & 4.3 & 17.4 & 284.8 \\
\hline 00035942 & angpt/4 & -0.3 & 53.9 & 37.8 & 4.1 & 20.1 & 260.6 \\
\hline 00049924 & angpt/4 & -0.4 & 52.1 & 33.0 & 3.9 & 20.6 & 244.3 \\
\hline 00018981 & $\operatorname{tgm} 1 / 1$ & 0.9 & 1.3 & 2.0 & 3.8 & 45.6 & 509.5 \\
\hline 00009477 & crema & 0.0 & 12.0 & 10.3 & 3.7 & 10.0 & 108.6 \\
\hline 00020192 & $p d e 4 b b$ & -0.1 & 37.4 & 29.3 & 3.6 & 25.4 & 266.8 \\
\hline 00018013 & gadd45aa & 0.3 & 46.9 & 49.9 & 3.5 & 23.9 & 220.8 \\
\hline 00040271 & rbp2a & 0.0 & 12.2 & 11.3 & 3.3 & 35.4 & 287.9 \\
\hline 00035774 & vegfab & -0.2 & 25.1 & 18.1 & 3.0 & 12.0 & 82.0 \\
\hline
\end{tabular}




\begin{tabular}{|c|c|c|c|c|c|c|c|}
\hline $\begin{array}{l}\text { Gene ID } \\
\text { cypCar }\end{array}$ & Gene & Log2FC M1 & RPKM C & RPKM M1 & Log2FC M2 & $\begin{array}{c}\text { RPKM C } \\
\text { M2 }\end{array}$ & RPKM M2 \\
\hline 00015586 & $\begin{array}{l}\text { rab26 or } \\
\text { rab37 }\end{array}$ & -0.4 & 8.9 & 6.0 & 3.0 & 9.1 & 58.9 \\
\hline 00043137 & cremb & 0.0 & 20.7 & 17.4 & 2.9 & 12.6 & 82.0 \\
\hline 00040375 & crema & 0.1 & 40.2 & 36.6 & 2.9 & 31.1 & 197.2 \\
\hline 00005288 & map3k15 & -0.2 & 25.6 & 19.6 & 2.8 & 12.8 & 77.0 \\
\hline 00032256 & $h s d 11 b 2$ & 0.0 & 17.9 & 13.9 & 2.7 & 35.2 & 167.4 \\
\hline 00011045 & soat1 & 0.1 & 18.7 & 17.5 & 2.7 & 17.6 & 95.5 \\
\hline 00005655 & $b t g 1$ & -0.2 & 119.3 & 87.8 & 2.7 & 77.8 & 426.6 \\
\hline 00011046 & soat1 & 0.0 & 19.6 & 16.9 & 2.7 & 18.3 & 99.5 \\
\hline 00018053 & pnp5a & 0.3 & 617.8 & 608.5 & 2.6 & 404.4 & 2060.3 \\
\hline 00007693 & eif4ebp3 & -0.1 & 241.5 & 189.8 & 2.6 & 192.3 & 950.2 \\
\hline 00000437 & eif4ebp3 & -0.3 & 23.6 & 15.8 & 2.2 & 22.0 & 93.2 \\
\hline 00032368 & tcim & -0.5 & 60.3 & 37.3 & 2.2 & 24.8 & 100.0 \\
\hline \multicolumn{8}{|c|}{ Significantly upregulated in both M2 and M1 but at least 2.5 log2 higher in M2 compared to M1 } \\
\hline 00001309 & cyr61/1 & 3.1 & 0.5 & 4.6 & 9.4 & 0.2 & 103.8 \\
\hline 00022158 & ramp2 & 2.0 & 2.3 & 7.8 & 6.4 & 1.8 & 100.9 \\
\hline 00035581 & dfna5a & 2.0 & 7.2 & 29.7 & 5.1 & 12.8 & 421.8 \\
\hline 00034978 & $\arg 2$ & 1.0 & 54.6 & 95.7 & 4.8 & 19.9 & 445.2 \\
\hline 00015701 & csrnp1a & 0.9 & 18.8 & 31.0 & 4.2 & 7.5 & 117.1 \\
\hline 00033214 & cremb & 1.0 & 24.7 & 41.7 & 3.6 & 15.6 & 164.5 \\
\hline 00025413 & adam 28 & 0.8 & 112.9 & 166.6 & 3.4 & 114.1 & 1016.7 \\
\hline 00024444 & lox & 0.4 & 34.2 & 38.8 & 3.2 & 12.0 & 83.9 \\
\hline \multicolumn{8}{|c|}{ Downregulated exclusively in M2 } \\
\hline 00023172 & $c x c / 11-1$ & 0.0 & 90.7 & 85.4 & -2.9 & 92.7 & 9.2 \\
\hline 00002590 & $\begin{array}{l}\text { cxcl11-like } \\
\text { or cxcl18b }\end{array}$ & -0.1 & 1429.4 & 1135.0 & -2.7 & 1257.8 & 163.7 \\
\hline 00023915 & $f 3 b$ & 0.2 & 195.0 & 197.5 & -2.3 & 151.0 & 23.7 \\
\hline \multicolumn{8}{|c|}{ Significantly downregulated in M2 and significantly upregulated in M1 } \\
\hline 00038694 & $\operatorname{tm} 4 s f 18$ & 0.5 & 35.1 & 42.0 & -3.2 & 90.4 & 9.3 \\
\hline 00027661 & pfkfb3 & 0.9 & 40.9 & 63.7 & -2.0 & 29.1 & 6.1 \\
\hline 00021651 & cxcr3.3 & 1.1 & 31.1 & 54.8 & -2.0 & 53.0 & 10.1 \\
\hline 00035137 & marcks/1a & 1.1 & 190.1 & 352.9 & -1.6 & 244.1 & 67.2 \\
\hline \multicolumn{8}{|c|}{ Significantly downregulated in both M2 and 2 but at least 2.5 log2 lower in M2 compared to M1 } \\
\hline 00048576 & $\operatorname{tm} 4 s f 1$ & -3.2 & 61.4 & 5.8 & -0.5 & 59.9 & 37.2 \\
\hline
\end{tabular}


Supplementary table 3.4 Primer sequences recognizing multiple paralogs of one gene that were used for RT-qPCR. Primer sequences are depicted with the gene abbreviation (Gene), Forward (FW) primer sequence, reverse (RV) primer sequence and Genbank accession number. ${ }^{\mathrm{a}} \mathrm{cypCar}$ numbers identify open reading frames in the draft carp genome (BioProject: PRJNA73579) which were confirmed by RNA sequencing

\begin{tabular}{|llll|}
\hline Gene & FW primer (5'-3') & RV primer (5' to 3') & $\begin{array}{l}\text { Genbank acc. } \\
\text { number }\end{array}$ \\
\hline 40s & CCGTGGGTGACATCGTTAC & TCAGGACATTGAACCTCACTGTC & AB012087 \\
\hline il1b & AAGGAGGCCAGTGGCTCTGT & CCTGAAGAAGAGGAGGCTGTCA & AJ245635 \\
\hline nos2 & AACAGGTCTGAAAGGGAATCCA & $\begin{array}{l}\text { CATTATCTCTCATGTCCAGAGTC } \\
\text { TCTTCT }\end{array}$ & AJ242906 \\
\hline saa & CCCAGGACAAGCCATTG & GCAGCATCATAGTTCCC & $\begin{array}{l}\text { cypCar_00037333 } \\
\text { cypCar_00036204 }\end{array}$ \\
\hline timp2b & TGGCAAAAAGGAATACCTG & CTCAGAGACTCCCAAGATTC & $\begin{array}{l}\text { cypCar_00030755 } \\
\text { cypCar_00034223 }\end{array}$ \\
\hline tgm2b & GCCTGGTATTTTGGACAGT & GCACTCAGCACTCTTGT & $\begin{array}{l}\text { cypCar_00030329- } \\
\text { cypCar_00034483 } \\
\text { cypCar_00041907 }\end{array}$ \\
\hline arg2 & GGAGACCTGGCCTTCAAGCATCT & TGATTGGCACGTCCAACT & AJ618955 \\
\hline
\end{tabular}




\section{Supplementary methods: Transciptome sequencing supports a striking conservation of macrophage polarization in fish}

We developed an analysis that quantifies which genes qualify as candidate M1 or as candidate M2 marker. This script was written in Python (version 3.7) and relies on the packages pandas [1] and matplotlib [2]. For the selection criteria we defined the averaged reads per kilobasepair per million reads (RPKM) over replicates as:

$$
\operatorname{RPKM}_{i}=\frac{1}{n_{\text {rep }}} \sum_{i_{\text {rep }}=0}^{n_{\text {rep }}}\left(\frac{\text { reads }_{i}}{\sum_{i=0}^{n}\left(\text { reads }_{i}\right) \cdot 10^{-6} \cdot \text { width }_{i} \cdot 10^{-3}}\right)_{i_{\text {rep }}}
$$

, where $\mathrm{RPKM}_{i}$ is the value of averaged RPKM for gene $i$ (we will refer to the averaged RPKM as the RPKM), reads $s_{i}$ the reads for gene $i, \sum_{i}=0^{n}$ reads $_{i}$ the sum of all the reads and width ${ }_{i}$ the width of the gene $i$ in basepairs. The candidate marker genes are selected based on the following criteria:

1. The gene is significantly regulated, which means that $p_{\text {adjusted }}<0.05$

2. The RPKM in control- or stimulated-samples are larger than 50 .

3. The $\log _{2}$ (fold) $>1.5$ with respect to the control. This means that the (nonlog transformed) fold change is larger than $\approx 2.82$.

4. The difference in $\log _{2}$ (fold) $>2$ between both stimulated groups. This means that the (non-log transformed) fold change fraction is larger than 4 .

The code presented in SC 1 takes the output data from DESeq2 [3], applies the above criteria and plots the results. As a bonus we included a picker which enables the end-user to click in the figure and display the genes around the click. The code consists of 2 functions (def main(), def plot_area(args)) and one class (class Picker) The function def main() consists of the following blocks of code:

1. line 10-19 Definition of parameters of the script. The values of the paths (path and output) are replaced by two placeholders and should be changed by the end-user to the correct locations.

2. line 27-33 The output data from DESeq2 [3] is loaded from the hard drive

3. line 36-37 When the data has a NaN value it is removed from the data-set. However, it might be that the gene-

expression in one data-set is undetermined and in the other is highly expressed, this data is still removed. The end-user should be aware of this issue and locate these genes manually. 
4. line 40 - 43 The data is merged based on GeneID (in our case cypCar number), the LPS stimulated set of genes is assigned the suffix _lps and the cAMP stimulated set of genes is assigned the suffix _ camp.

5. line 48-69 Three data-sets are formed; lps_significant, camp_significant and both_significant. In the first data-set only the LPS-genes are significantly regulated, and the RPKM in control- or stimulated-samples is larger than 50. The second data-set is based on the same criteria, but for the genes of cAMP stimulated macrophages. In the last data-set genes are significantly regulated by both stimuli, and one of the four RPKMs is larger than 50.

6. line 71-74 The three data-sets are written to the hard drive. Theses dataset can be used to verify that the code performed as expected.

7. line 77-176 Based on the previous three data-sets eight data-sets are formed. As an example we will discuss the first four data-sets, which consists of all the potential markers for the LPS stimulant:

(a) lines 77-88 The set lps_marker consists of all the potential LPS markers which are only significantly regulated in LPS and fit the following criteria; the log2 fold change of LPS with respect to the control $>1.5$, the log 2 fold change of cAMP with respect to the control $>-1.5$ and the difference between the difference between the two $>2$. Or in the case of downregulated genes: the log2 fold change of LPS with respect to the control $<-1.5$, and the log2 fold change of cAMP with respect to the control $<1.5$ and the difference between the two $<-2$. Note that $\log _{2}(a)-\log _{2}(b)=\log _{2}(a / b)$

(b) lines 89-100 The set lps_marker_both consists of all the potential LPS markers which are only significantly regulated in LPS and fit the following criteria; the log2 fold change of LPS with respect to the control $>1.5$, the log2 fold change of cAMP with respect to the control $<-1.5$ and the difference between the difference between the two $>2$. Or; the log2 fold change of LPS with respect to the control $<-1.5$, and the log2 fold change of cAMP with respect to the control $>1.5$ and the difference between the two $<-2$. This last step includes all the significantly down-regulated genes.

(c) lines 101 - 112 The set lps_marker_sig_both consists of all the potential LPS markers which are significantly regulated in both LPS and cAMP and fit the following criteria; the log2 fold change of LPS with respect to the control $>1.5$, the log2 fold change of cAMP with respect to the control $>-1.5$ and the difference between the difference between the two $>2$. Or in the case of downregulated genes the log2 fold change of LPS with respect to the control $<-1.5$, and the log2 fold change of cAMP with respect to the control $<1.5$ and the difference between the two $<-2$. This set consists of markers for LPS 
where the genes from the LPS data-set are more than $1.5 \log 2$ fold regulated with respect to the control, but the data-set from the cAMP are not more than $1.5 \log 2$ fold regulated with respect to the control. The difference between the log2 fold change of the genes from both data-sets should still be more than 2 .

(d) lines 113 - 112 The set lps_marker_sig_both consists of all the potential LPS markers which are both significantly regulated in LPS and cAMP and fit the following criteria; the log2 fold change of LPS with respect to the control $>1.5$, the log2 fold change of cAMP with respect to the control $<-1.5$ and the difference between the difference between the two $>2$. Or in the case of downregulated genes the log2 fold change of LPS with respect to the control $<-1.5$, and the log2 fold change of cAMP with respect to the control $>1.5$ and the difference between the two $<-2$. This set consists of markers that are both regulated in the data-set of LPS and cAMP. However, the regulation of the gene in both data-sets is opposite in direction (e.g when the log2 fold change of LPS is positive that of cAMP is negative). This set consists of the shaded areas in figure 3 .

8. lines $178-240$ The eight data-sets that are formed as a result of the filtering are split into data-sets containing upregulated or downregulated genes and written to a single excel file. This file should again be verified by the end-user to make sure that the above filtering is applied correctly.

9. lines $242-309$ The data-sets are plotted, the end result forms figure 3 .

The function def plot_area (args) (lines 312 - 370) is used to draw the shaded areas in figure 3 based on the input of the user (lines 10-19). Finally the class Picker is used to determine where the user clicked in the figure and presents the user with all the genes around the click. The filtering of these genes occurs on lines $397-400$. Finally on lines $409-410$ the function def main () is called to start the script.

Source Code 1: Analysis code to select potential markers.

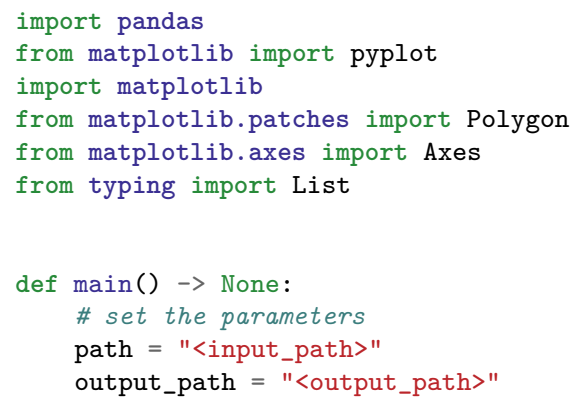




\section{Chapter 3}

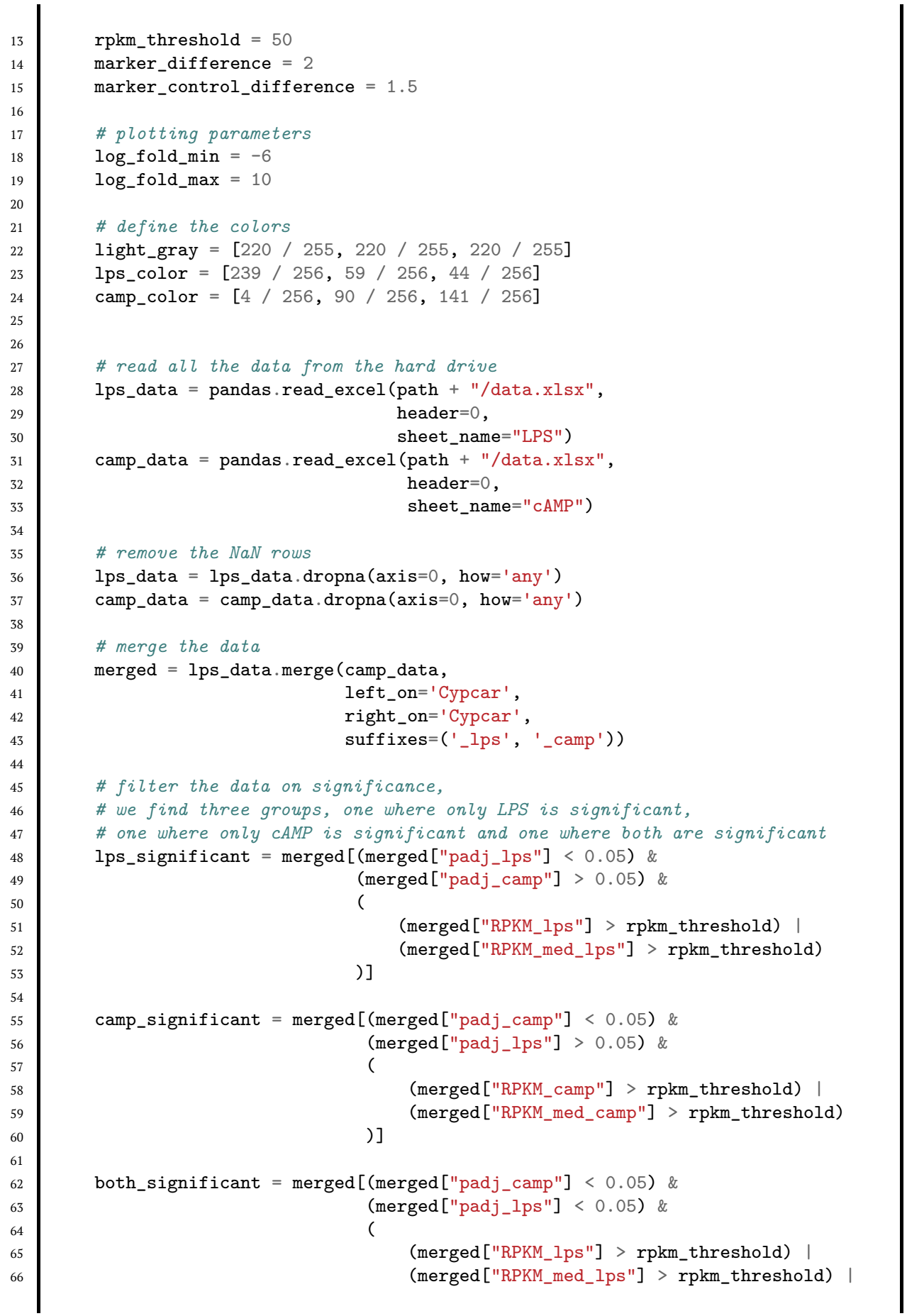


(merged["RPKM_camp"] > rpkm_threshold) | )]

\# write all the significant data to the hard-drive

lps_significant.to_excel (output_path + "/lps_output.xlsx")

camp_significant.to_excel (output_path + "/camp_output.xlsx")

both_significant.to_excel (output_path + "/both_output.xlsx")

\# filter based on the critera given above

lps_marker = lps_significant [

(

(lps_significant ["log2FoldChange_lps"] >

$\hookrightarrow$ marker_control_difference) \&

(lps_significant ["log2FoldChange_camp"] >

$\hookrightarrow$-marker_control_difference) \&

((lps_significant ["log2FoldChange_lps"] -

) 1

$\hookrightarrow$ lps_significant ["log2FoldChange_camp"] > marker_difference))

(

(lps_significant ["log2FoldChange_lps"] <

$\hookrightarrow \quad$-marker_control_difference) \&

(lps_significant ["log2FoldChange_camp"] <

$\hookrightarrow$ marker_control_difference) \&

((lps_significant ["log2FoldChange_lps"] -

$\hookrightarrow$ lps_significant ["log2FoldChange_camp"] $)<$-marker_difference)

]

lps_marker_both $=$ lps_significant [

(

(lps_significant ["log2FoldChange_lps"] >

$\hookrightarrow$ marker_control_difference) \&

(lps_significant ["log2FoldChange_camp"] <

$\hookrightarrow \quad$-marker_control_difference) \&

( (lps_significant ["log2FoldChange_lps"] -

) 1

$\hookrightarrow$ lps_significant ["log2FoldChange_camp"]) > marker_difference)

(

(lps_significant ["log2FoldChange_lps"] <

$\hookrightarrow \quad$-marker_control_difference) \&

(lps_significant ["log2FoldChange_camp"] >

$\hookrightarrow$ marker_control_difference) \&

( (lps_significant ["log2FoldChange_lps"] -

$\hookrightarrow$ lps_significant ["log2FoldChange_camp"] ) < -marker_difference)

]

lps_marker_both_sig = both_significant [

(

(both_significant ["log2FoldChange_lps"] >

$\hookrightarrow$ marker_control_difference) \&

(both_significant ["log2FoldChange_camp"] >

$\hookrightarrow$-marker_control_difference) \&

( (both_significant ["log2FoldChange_lps"] -

$\hookrightarrow$ both_significant ["log2FoldChange_camp"]) > marker_difference) 


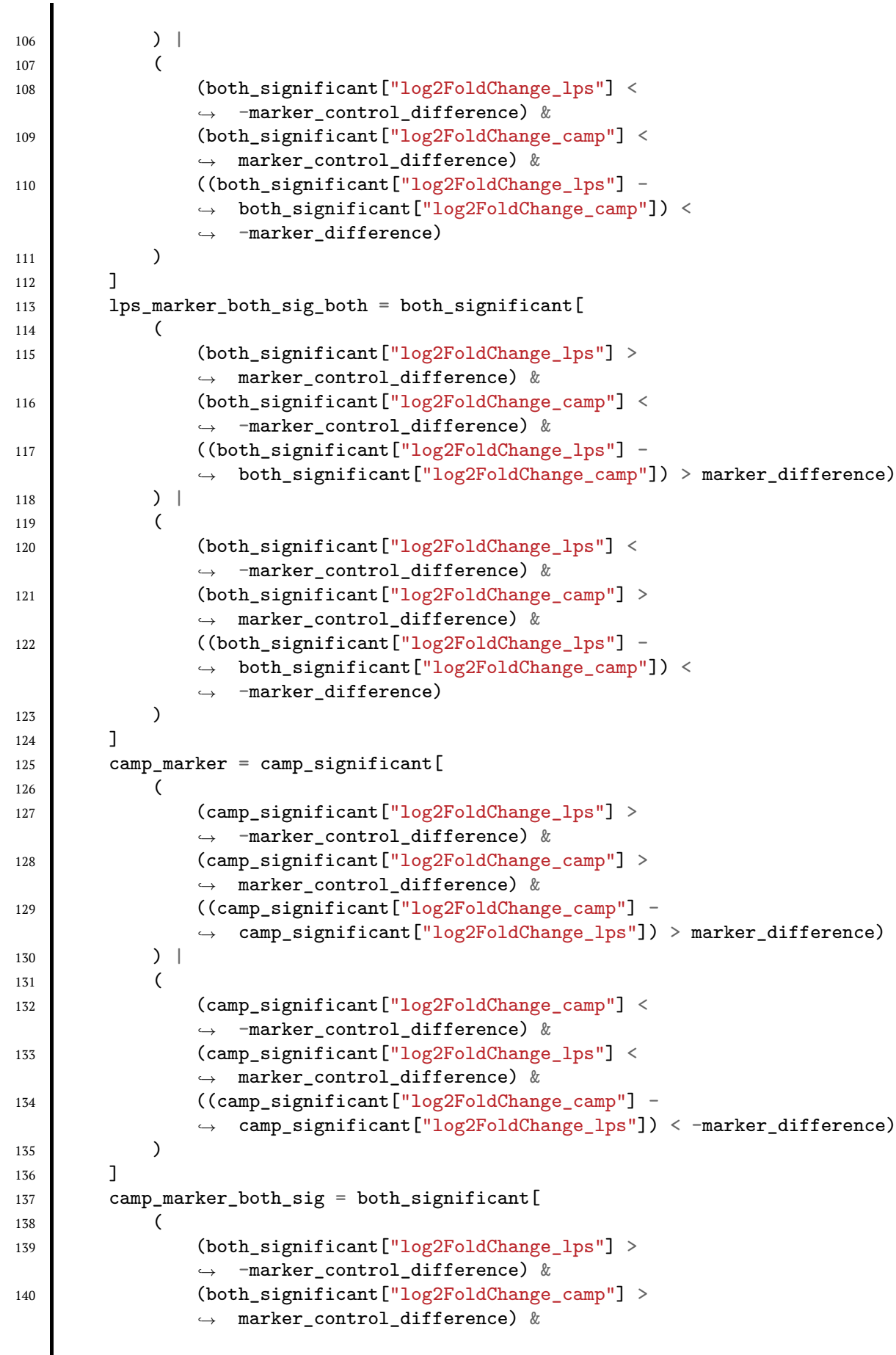




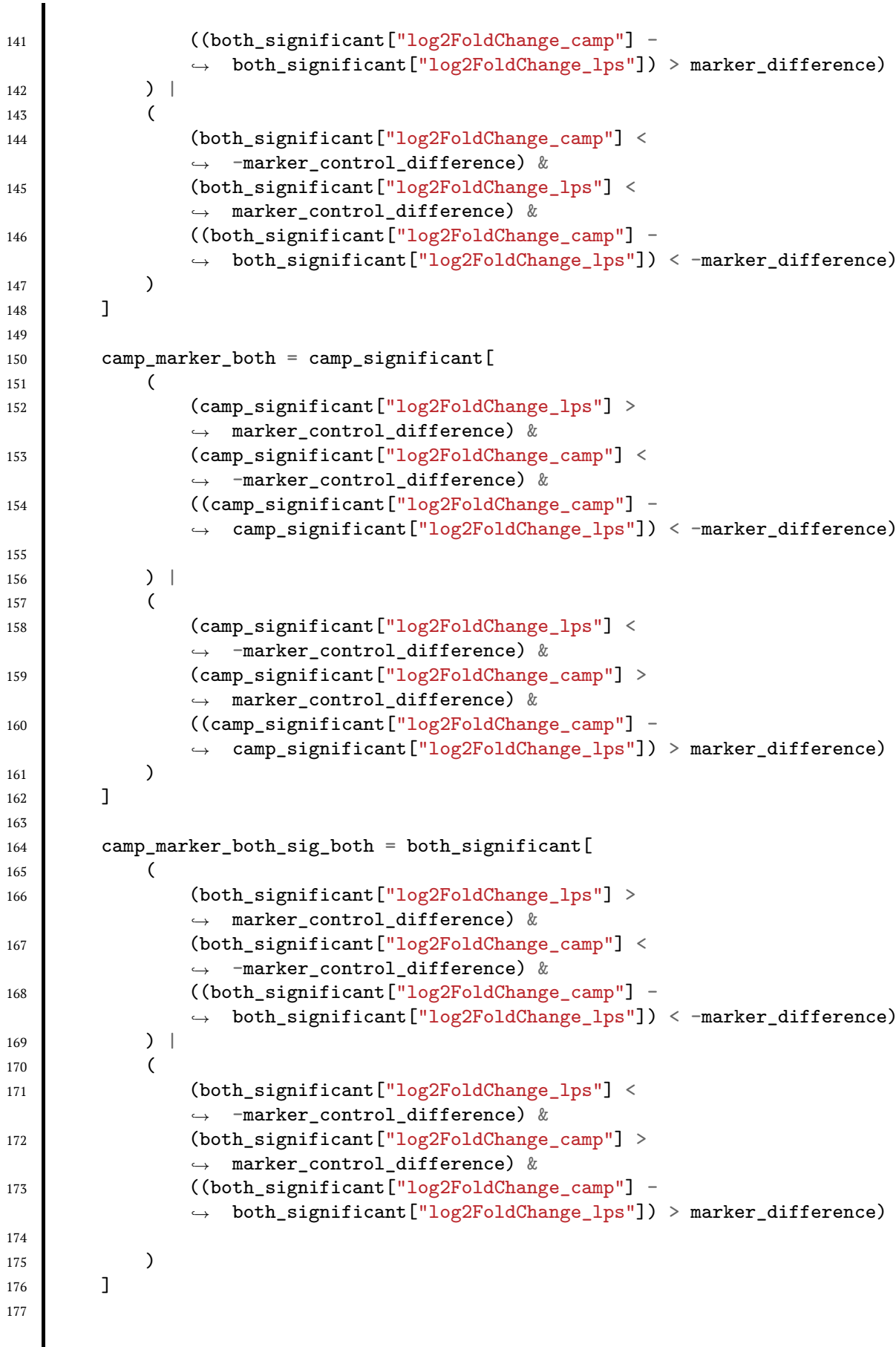




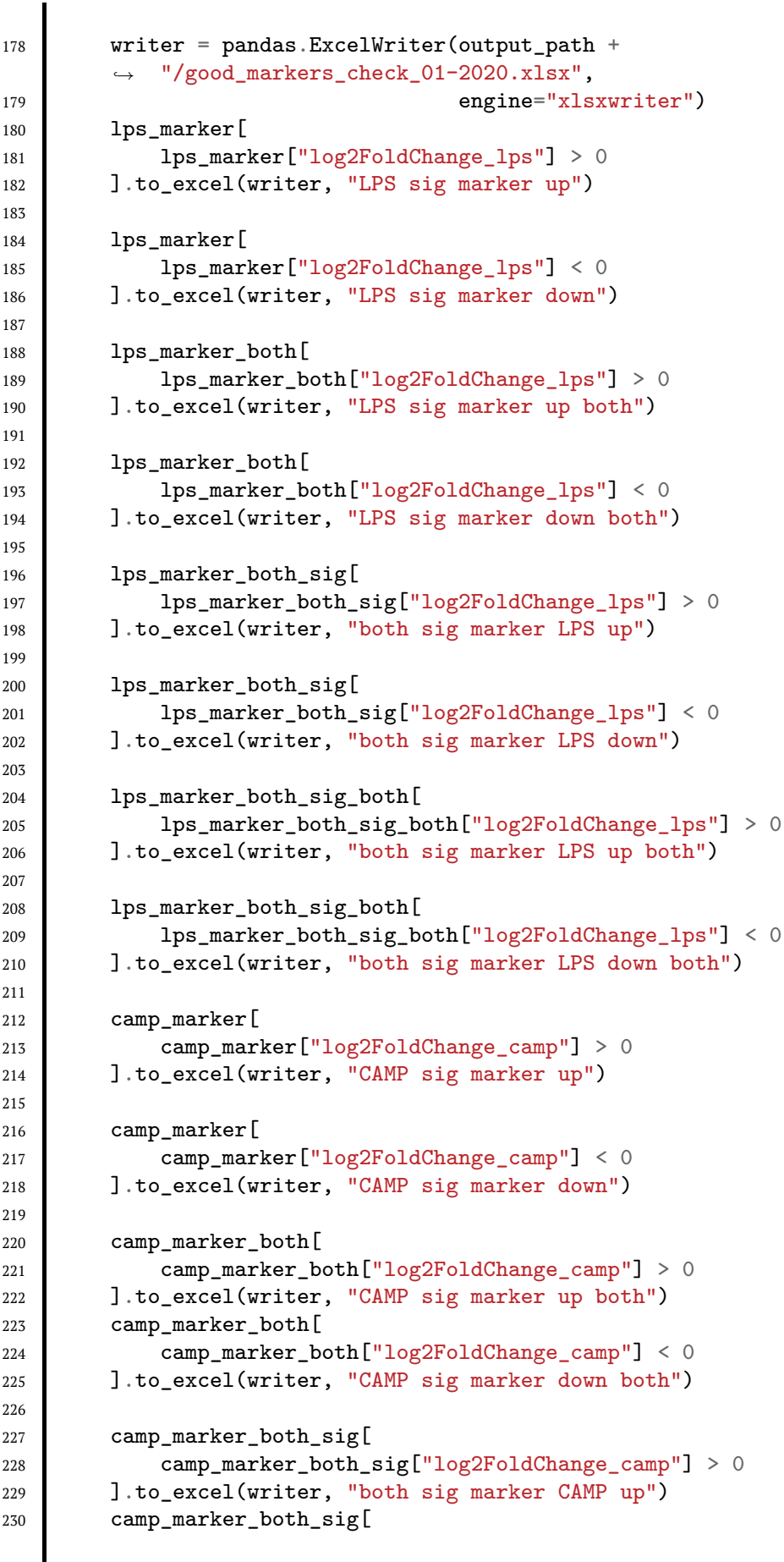




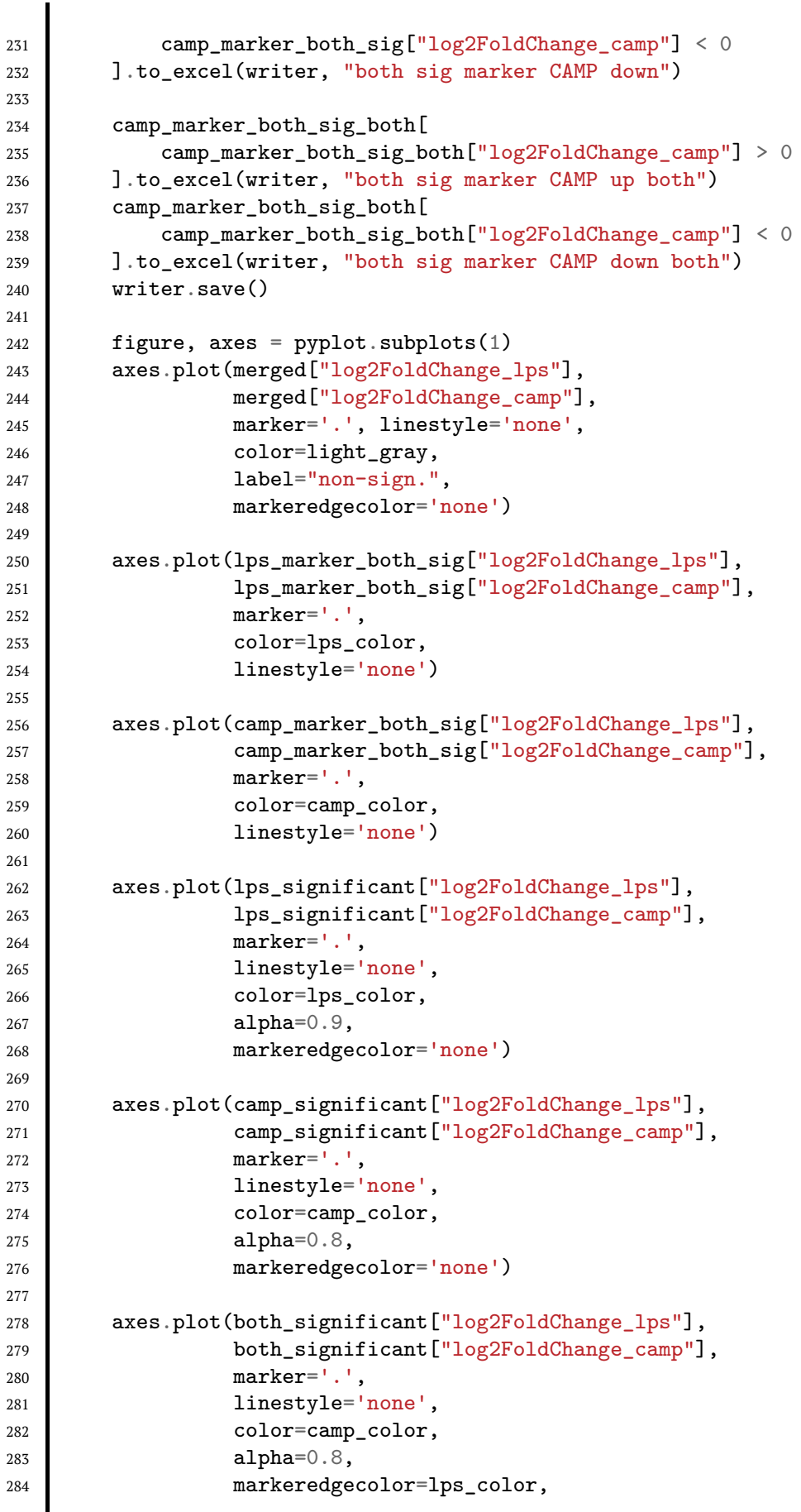




\section{Chapter 3}

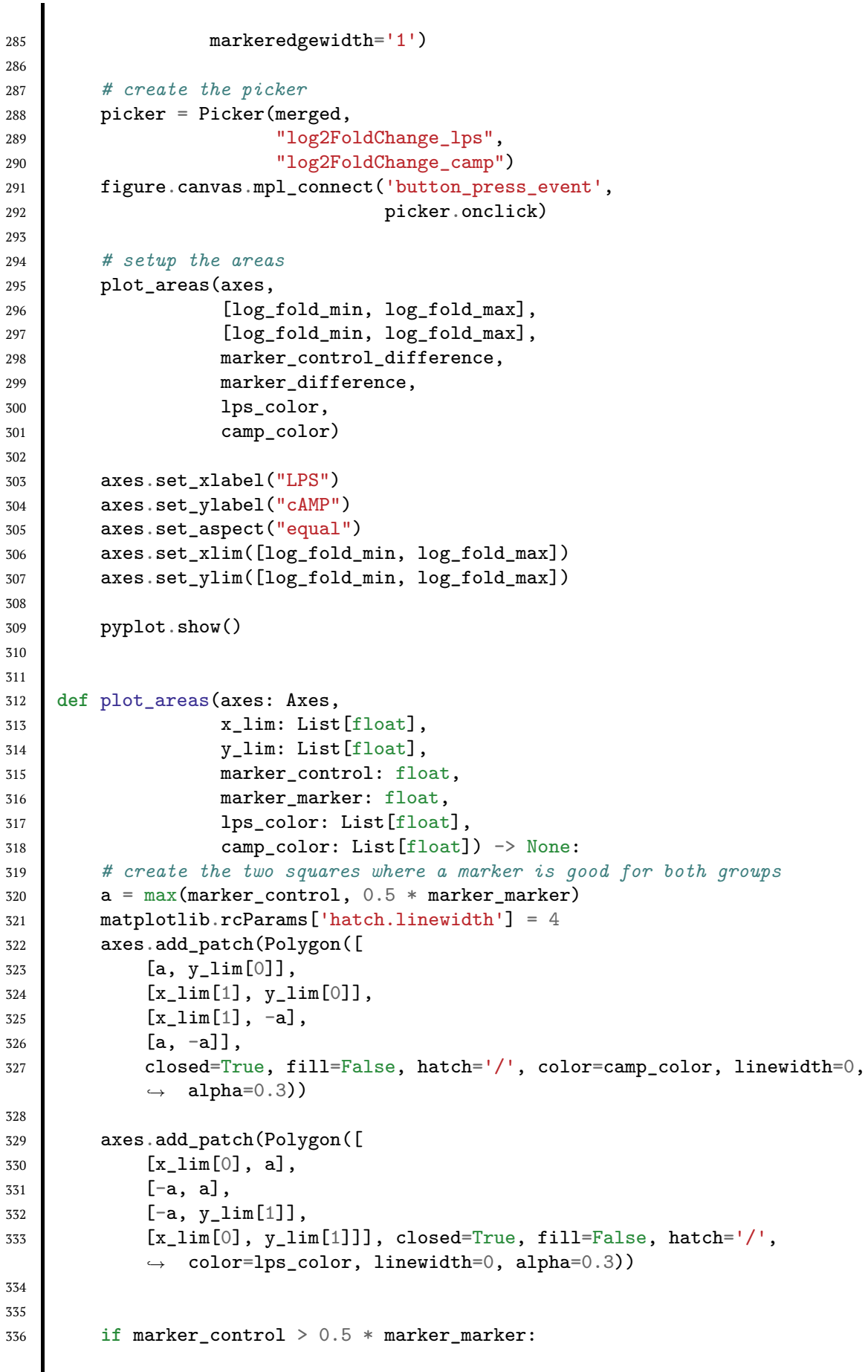




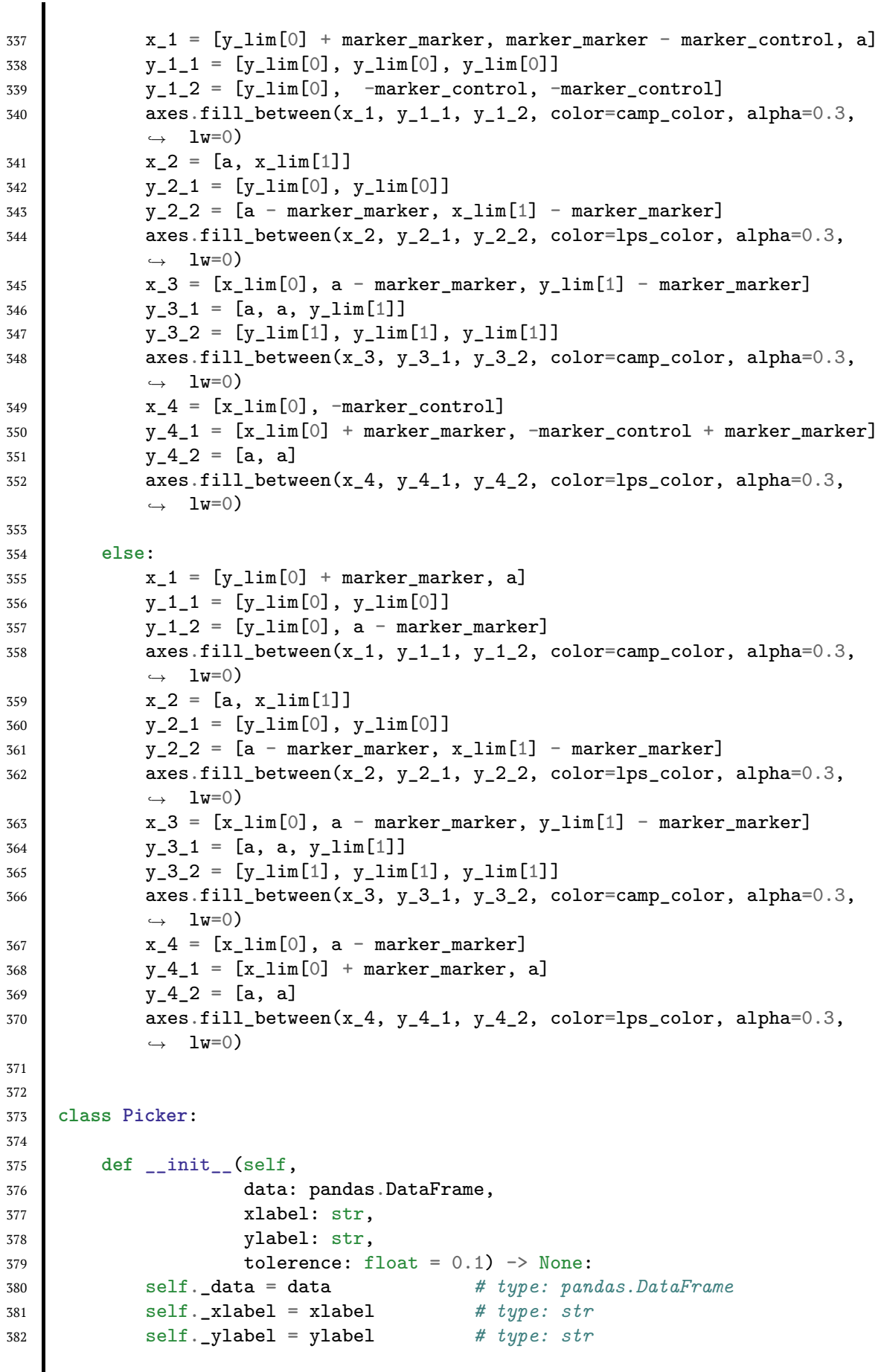




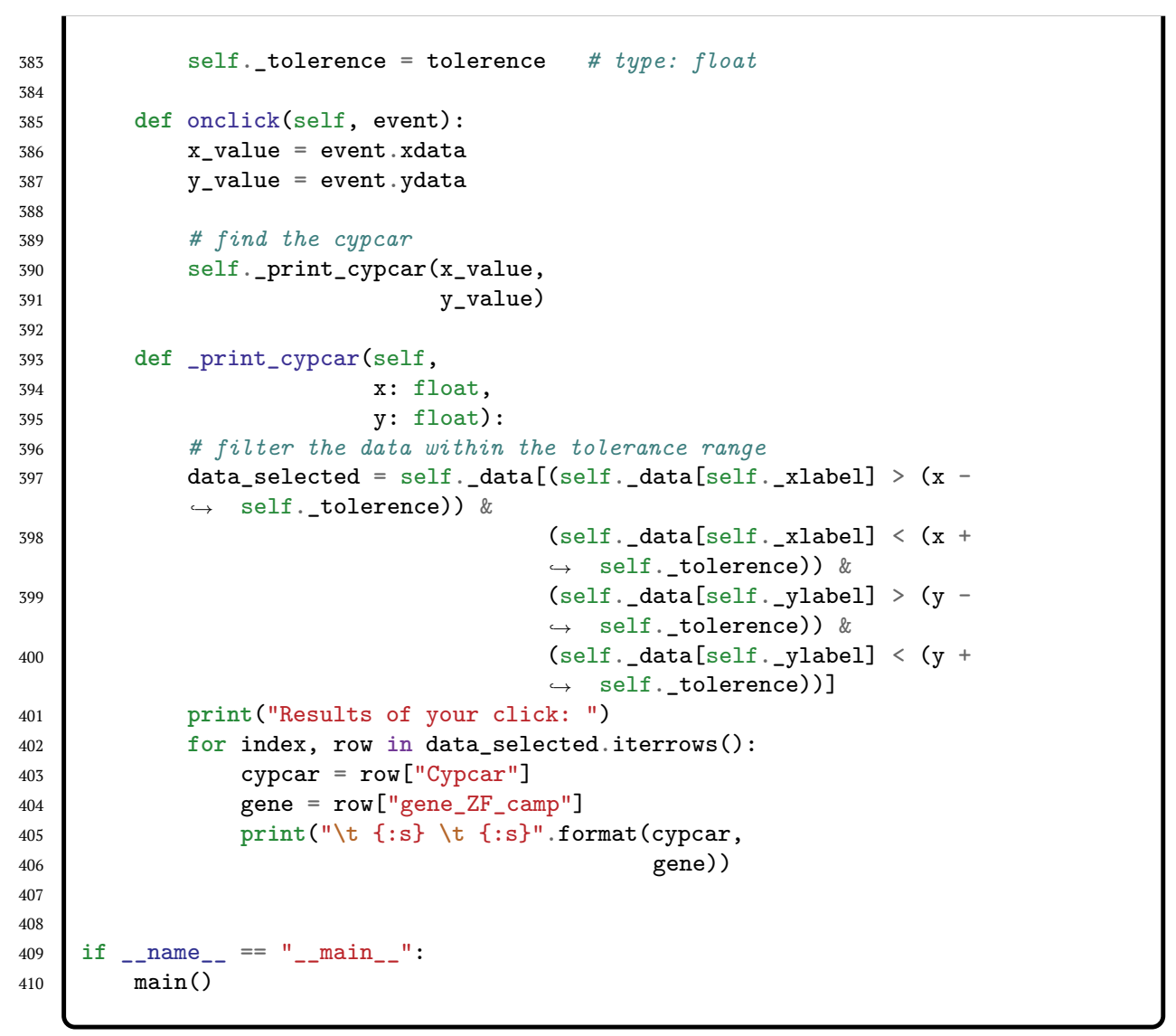

\section{References}

[1] Wes McKinney (2010) Data Structures for Statistical Computing in Python. Proc of the 9th Python in Science Conf Ed. by Stéfan van der Walt and Jarrod Millman. 51 -56.

[2] J. D. Hunter (2007) Matplotlib: A 2D graphics environment. Computing in Science \& Engineering 9.3;90-95. doi: 10.1109/MCSE.2007.55.

[3] Michael I. Love, Wolfgang Huber, and Simon Anders (2014) Moderated estimation of fold change and dispersion for RNA-seq data with DESeq2. Genome Biol 15.12. doi: 10.1186/s13059014-0550-8. 
Conservation of macrophage polarization in fish 



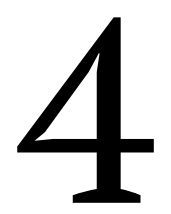

\section{Paralogs of common carp granulocyte colony- stimulating factor (G-CSF) have different functions regarding development, trafficking and activation of neutrophils}

Fumihiko Katakura ${ }^{1,4}$, Kohei Nishiya ${ }^{1,4}$, Annelieke S. Wentzel ${ }^{2}$, Erika Hino $^{1}$, Jiro Miyamae ${ }^{1}$, Masaharu Okano ${ }^{1}$, Geert F. Wiegertjes ${ }^{2,3}$, Tadaaki

Moritomo $^{1}$

${ }^{1}$ Laboratory of Comparative Immunology, Department of Veterinary Medicine, Nihon University, Fujisawa, Kanagawa 252-0880, Japan

${ }^{2}$ Cell Biology and Immunology Group, ${ }^{3}$ Aquaculture and Fisheries Group, Wageningen Institute of Animal Science, Wageningen University \& Research, Wageningen, The

Netherlands

${ }^{4}$ Authors with equal contribution

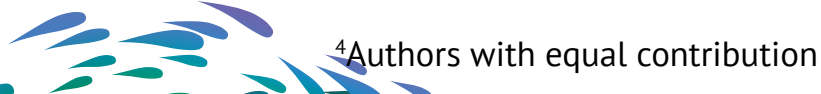




\begin{abstract}
Mammalian granulocyte colony-stimulating factor (G-CSF; CSF3) is a primary cytokine that promotes the development, mobilization, and activation of neutrophils and their precursors. Teleosts have been reported to possess two paralogs as a likely result of the teleost-wide whole genome duplication (WGD) event, but functional divergence of G-CSF paralogs remains poorly understood. Common carp are an allotetraploid species owing to an additional WGD event in the carp lineage and here, we report on genomic synteny, sequence similarity, and phylogeny of four common carp G-CSF paralogs ( $g$-csfa1 and $g$-csfa2; $g$-csfb1 and $g$-csfb2). $G$-csfa 1 and $g$-csfa2 show differential and relatively high gene expression levels, while $g$ - $c s f b 1$ and $g$-csfb2 show low basal gene expression levels in most tissues. All paralogs are expressed higher in macrophages than in other leukocyte sub-types and are highly up-regulated by treatment of macrophages with mitogens. Recombinant G-CSFa1 and G-CSFb1 both promoted the proliferation of kidney hematopoietic cells, while only G-CSFb1 induced the differentiation of kidney cells along the neutrophil-lineage. Colony-forming unit assays revealed that G-CSFb1 alone stimulates the formation of CFU-G colonies from head- and trunk-kidney whereas the combination of G-CSFa1 and G-CSFb1 stimulates the formation of both CFU-G and CFU-GM colonies. Recombinant G-CSFa1 and G-CSFb1 also exhibit chemotactic activity against kidney neutrophils and up-regulation of cxcr1 mRNA expression was highest in neutrophils after G-CSFb1 stimulation. Furthermore, G-CSFb1 more than G-CSFa1 induced priming of kidney neutrophils through upregulation of a NADPH-oxidase component $\mathrm{p} 47^{\text {phox }}$. In vivo administration of G-CSF paralogs increased the number of circulating blood neutrophils of carp. Our findings demonstrate that gene duplications in teleosts can lead to functional divergence between paralogs and shed light on the sub-functionalization of G-CSF paralogs in cyprinid fish.
\end{abstract}




\section{Introduction}

Granulocyte colony-stimulating factor (G-CSF), also called colony-stimulating factor 3 (CSF3), is a primary cytokine that promotes the proliferation, differentiation and survival of neutrophil progenitors and enhances trafficking and immunological functions of mature neutrophils in mammals [1]. Human G-CSF is produced mainly by monocytes and macrophages [2], but is also produced by fibroblasts [3], endothelial cells [4] and bone marrow stromal cells [5]. Although healthy individuals express low G-CSF protein levels in serum, remarkable elevations of G-CSF production can be induced by several inflammatory stimuli, including increased presence of pro-inflammatory cytokines and LPS during infections [6-8]. Effects are mediated by the binding of G-CSF with its cognate receptor G-CSFR on neutrophils and their progenitors, activating downstream signaling cascades and thereby influencing subsequent gene expression and cellular immune responses (reviewed in [1]). Mice lacking G-CSF/G-CSFR signaling (G-csf-deficient or G-csfrdeficient mice) exhibit a reduction in myeloid progenitors and impaired neutrophil mobilization into the circulation, resulting in chronic neutropenia $[9,10]$. This suggests that G-CSF is a major regulator of neutrophil development and contributes to the regulation of multipotent hematopoietic progenitors. At the same time, G-CSF also influences the phenotype and function of mature neutrophils and does so by modulating expression of for example chemokine receptors, up-regulating phagocytosis and production of reactive oxygen species (ROS) and enhancing bactericidal activity of neutrophils [11].

G-CSF was first purified and characterized in mice [12], only later followed by studies in non-mammalian vertebrates such as chicken [13], African-clawed frog [14], and a number of teleost fish species including flounder, fugu, green-spotted pufferfish [13], black rockfish [15], and zebrafish [16]. Owing to a teleost-specific whole genome duplication (WGD) event [17], teleost can generally be expected to express two G-CSF paralogs, type A (G-CSFa) and type B (G-CSFb), which may not necessarily have the same function. Indeed, zebrafish express an $A$ and $B$ paralog and earlier studies suggest that both G-CSFa and G-CSFb are required for hematopoietic stem cell (HSC) emergence and expansion of primitive and mature neutrophils and macrophages in vivo and in vitro [16]. G-csfr morphants were affected on early myeloid cell migration and development, but had functionally normal myeloid cells [18]. Zebrafish G-CSFb was involved in neutrophil mobilization towards an injury site [19], but the contribution of G-CSFa remained unclear. Therefore the 
exact role of teleost G-CSF paralogs as regulators of diverse markers of neutrophil activation and/or regulators of multipotent hematopoietic progenitor development has remained unresolved.

In this study, we report on the molecular and functional characterization of G-CSF paralogs from the common carp. The close kinship of zebrafish and carp [20] allows for comparative use of genetic information from the well-described zebrafish genome whereas the large size of carp allowed us to perform cell type specific gene expression and ex vivo functional studies on large number of cells. Because common carp is an allotetraploid species owing to an additional WGD event in the carp lineage [21], we report on the cloning and molecular characterization of two type A copies ( $g$-csfa1 and $g$-csfa2) and two type B copies ( $g$-csfb1 and $g$-csfb2). For functional characterization we chose to produce recombinant proteins for two G-CSF paralogs particularly highly expressed in macrophages (G-CSFa1 and G-CSFb1) and examined their ability to drive proliferation, differentiation and colony-formation of carp hematopoietic kidney cells along the neutrophil lineage. We also studied the effect of these G-CSF paralogs on neutrophil function including phenotype, chemotaxis and production of ROS. In vivo effects of G-CSF paralogs on circulating blood neutrophils were further investigated. We discuss the functions of teleost G-CSF regarding development, trafficking and activation of neutrophils and discuss the importance of studying paralogs of granulocyte colony-stimulating factor.

\section{Materials and methods}

\section{Animals}

Common Carp (Cyprinus carpio L.) were kept at Nihon University (NU) and at Wageningen University (WU). Carp weighing $40-100 \mathrm{~g}$ (10 to $15 \mathrm{~cm}$ in length) were purchased from commercial farms and reared at NU, Japan. Fish were kept at 23 $25^{\circ} \mathrm{C}$ in a recirculation system with filtered water disinfected by ultraviolet light, fed with pelleted dry food (Hikari, Kyorin CO., LTD., Japan) daily and acclimated to this environment for at least three weeks prior to use for all experiments except Figure 4.2 - 4.4. Carp were also bred and reared in the Aquatic Research Facility of WU, the Netherlands. Here, carp were raised at $23^{\circ} \mathrm{C}$ in recirculating UV-treated tap water, fed pelleted dry food daily (Skretting, Nutreco) and utilized for experiments in Figure 4.2 - 4.4. Since G-CSF paralogs of Asian and European common carp show 
very high sequence identity (98 to 100\%), we combined data from NU and WU. Experiments were performed in accordance with the guidelines of $\mathrm{NU}$ and $\mathrm{WU}$ and with approval of the animal experimental committee of WU.

Isolation of carp tissues and leukocytes and purification of leukocyte sub-types such as B cells, granulocytes, macrophages, thymocytes and thrombocytes

For tissue and cell isolation, carp were anesthetized with 0.01\% Benzocaine (SigmaAldrich) or Tricaine Methane Sulfonate (TMS, Crescent Research Chemicals, Phoenix, USA), bled from the caudal vein and euthanized.

Leukocytes were obtained from kidney (head and/or trunk kidney) and spleen. Cell suspensions were obtained by macerating tissues on a sterile mesh in $10 \mathrm{~mL}$ of Eagle's minimal essential medium (MEM, Nissui, Tokyo, Japan). Cells were collected by centrifugation at $250 \times \mathrm{g}$ for $5 \mathrm{~min}$ at $4^{\circ} \mathrm{C}$, re-suspended in $5 \mathrm{~mL}$ of MEM, layered onto a Percoll $\left(1.075 \mathrm{~g} / \mathrm{cm}^{3}\right.$, GE healthcare) and centrifuged at $430 \times \mathrm{g}$ for $20 \mathrm{~min}$ at $4^{\circ} \mathrm{C}$. Cells at the medium/Percoll interface (mononuclear cells) were harvested, washed twice with MEM by centrifugation, re-suspended with E-RDF medium (Kyokuto Pharm. Ind. Co.,Ltd., Tokyo, Japan) containing 20\% fetal bovine serum and $2.5 \%$ carp serum (E-RDF20/2.5) and passed through $40 \mathrm{~mm}$ filter to remove aggregate.

Peripheral blood leukocytes (PBL) were obtained from carp blood. In short, $1 \mathrm{~mL}$ of blood was withdrawn from the caudal vein from fish with heparinized syringe, transferred to $9 \mathrm{~mL}$ of ice-cold MEM, layered onto a Percoll (1.075) and centrifuged at $430 \times \mathrm{g}$ for $20 \mathrm{~min}$ at $4^{\circ} \mathrm{C}$ without brakes. Cells at the medium/Percoll interface were harvested, washed twice with MEM by centrifugation and re-suspended with E-RDF20/2.5.

Kidney neutrophils were isolated as described previously [22] with minor modifications. Briefly, trunk kidney cells were layered onto a Percoll discontinuous gradient $\left(1.080\right.$ and $\left.1.100 \mathrm{~g} / \mathrm{cm}^{3}\right)$ and centrifuged at $430 \times \mathrm{g}$ for $20 \mathrm{~min}$ at $4^{\circ} \mathrm{C}$. Cells at the 1.080/1.100 interface (neutrophils and erythrocytes) were harvested after complete removal of cells at the upper phase and then washed once. The neutrophil/ erythrocyte pellet was treated with $1 \times$ red blood cell lysis buffer $\left(150 \mathrm{mM} \mathrm{NH}_{4} \mathrm{Cl}, 10\right.$ $\mathrm{mM} \mathrm{KHCO}_{3}, 0.1 \mathrm{mM}$ EDTA). Cells were washed twice with MEM by centrifugation and re-suspended with appropriate medium for each experiment. The purity of the 
neutrophils was verified to be $>92 \%$ by a flow cytometry using a BD FACS Canto (BD Biosciences) and a peroxidase staining according to a DAB oxidization.

Thymocytes [23], macrophages [24], neutrophilic granulocytes [25] were obtained as previously described. Magnetic activated cell sorting (MACS) was used to isolate B cells and thrombocytes from peripheral blood leukocytes (PBLs) using anticarp IgM (WCI12, [26]) and anti-carp thrombocytes (WCL6, [27]) antibodies and neutrophilic granulocytes from trunk kidney (using monoclonal antibody TCL-BE8; [25]). The purity of the sorted cells was verified to be $>98 \%$ by flow cytometry using a BD FACS Canto A (BD Biosciences).

\section{Identification and in silico analysis of carp G-CSFs}

Genomic loci of carp G-CSF were predicted by the Augustus gene prediction server using information on genes (med24, psmd3 and kpnb1) known to be neighboring $\mathrm{G}-\mathrm{CSF}$ in several other species. Primers were designed against carp genomic sequences encoding putative carp G-CSFs. The complete list of primers used for PCR, RACE PCR, qRT-PCR and recombinant protein expression are listed in Supplementary tables S4.2, S4.3 and S4.4. PCR reactions were performed using cDNA from carp kidney and heart with PrimeSTAR HS DNA polymerase (Takara, Shiga, Japan). Generated amplicons were gel purified using the FastGene Gel/PCR Extraction kit (Nippon genetics, Tokyo, Japan), ligated into the pMD20-T vector (Takara) using the 10×A-attachment Mix (Toyobo, Osaka, Japan) and the Ligation high ver. 2 (Toyobo) and transformed into the competent Escherichia coli DH5 $\alpha$. Positive colonies were identified by colony PCR using the vector specific M13 RV and M4 primers, plasmids isolated using the FastGene Plasmid Mini kit (Nippon genetics) and inserts sequenced using a BigDye terminator v3.1 cycle sequencing kit (Applied Biosystems) and an ABI PRISM 3130 Genetic Analyzer (Applied Biosystems). Sequence analysis was performed using the Genetyx version 11 (Genetic Information Processing Software) and sequences aligned and analyzed using BLAST programs.

G-CSF protein sequences from fish, amphibian and mammals were aligned using Clustal Omega software (EMBL-European Bioinformatics Institute). Signal peptide regions of respective G-CSF proteins were estimated using the SignalP 4.0 server (http://www.cbs.dtu.dk/services/SignalP/) and conserved motifs were predicted using the SMART server (http://smart.embl-heidelberg.de/). Phylogenetic analysis 
was conducted using the MEGA version 6 software using the neighbor-joining (NJ) method and the Poisson method, and bootstrapped 1,000 times, with values expressed as percentages. The full-length sequences of carp G-CSFa1, G-CSFa2, G-CSFb1 and G-CSFb2 (accession number: MG882495, MG882496, MG882497 and MG882498, respectively) have been submitted to GenBank.

\section{RT-qPCR analysis of gene expression of carp G-CSF paralogs in healthy carp tissues, different cell types and macrophages stimulated with mitogens}

To investigate basal gene expression levels of G-CSF paralogs, tissues and leukocytes were collected from healthy carp (detailed in the figure legends), then total RNA was isolated using the RNeasy kit (QIAGEN) including on-column DNase treatment according to the manufacturer's instruction and stored at $-80^{\circ} \mathrm{C}$. cDNA was synthesized with SuperScript III First Strand Synthesis System (Invitrogen) according to manufacturer's instructions. Real-time quantitative PCR (RT-qPCR) was performed with a Rotor-Gene 6000 (Corbett Research) using ABsolute QPCR SYBR Green Mix (Thermo Scientific). Fluorescence data from RT-qPCR experiments were analyzed using Rotor-Gene software v1.7. The take-off value for each sample and the average reaction efficiencies $(E)$ for each primer set were obtained upon Comparative Quantitation Analysis from Rotor Gene Software. The relative expression ratio $(R)$ of target genes were calculated based on the average $E$ and relative to the $\mathrm{s} 11$ protein of the 40 s subunit as a reference gene. Take-off values of samples in which genes of interest were non-detectable were given an arbitrary take-off value of 32 .

\section{Generation of recombinant carp G-CSFa1 and G-CSFb1}

The portions of the carp G-CSFa1 and G-CSFb1 sequences corresponding to the mature, signal sequence-cleaved peptides were PCR amplified from carp kidney and heart cDNA using primers designed to meet the requirements of the pET$16 \mathrm{~b}$ expression vector (Novagen). The resulting PCR products were ligated into pMD20-T vector, digested with two restriction enzymes, NdeI and BamHI, isolated by gel electrophoresis, ligated into the pET-16b which carry an N-terminal 10x Histag, transformed into competent E. coli DH5 $\alpha$. Plasmids containing the in frame insert of carp G-CSFa1 or G-CSFb1 were transformed into the T7 polymerase expressing Rossetta-gami B (DE3) pLysS E. coli (Novagen), induced with appropriate IPTG and the optimal induction times and temperatures deduced in pilot runs. The bacteria were scaled up accordingly. 
Recombinant carp G-CSFa1 and G-CSFb1 were purified from bacterial cells using NiNTA agarose (Qiagen, Hilden, Germany) according to the manufacturer's procedure. Briefly, transformed E. coli cells were grown in $100 \mathrm{~mL} \mathrm{LB}$ medium containing 50 $\mathrm{mg} / \mathrm{mL}$ ampicillin and $30 \mathrm{mg} / \mathrm{mL}$ chloramphenicol to density of $\mathrm{OD}_{600}=0.5$ at $37^{\circ} \mathrm{C}$, and then cooled on ice. Expression of recombinant G-CSFa1 was induced by addition of $0.5 \mathrm{mM}$ IPTG at $37^{\circ} \mathrm{C}$ for $4 \mathrm{~h}$, and expression of recombinant G-CSFb1 was induced by addition of $0.25 \mathrm{mM} \mathrm{IPTG}$ at $25^{\circ} \mathrm{C}$ for $8 \mathrm{~h}$. After shaking the cultures, cells were harvested, lysed in the lysis buffer (20 mM sodium phosphate, $500 \mathrm{mM}$ $\mathrm{NaCl}, 50 \mathrm{mM}$ imidazole, $\mathrm{pH}$ 7.4, $0.1 \%$ Triton-X and protease inhibitor cocktail) and sonicated. The insoluble materials were removed by centrifugation at $9,600 \times g$ for $20 \mathrm{~min}$ and the supernatants were incubated with $500 \mu \mathrm{L}$ of Ni-NTA agarose slurry at $4^{\circ} \mathrm{C}$ for $1-2$ hours with gentle rotation. The resin was then washed with $30 \mathrm{~mL}$ of the wash buffer (20 mM sodium phosphate, $500 \mathrm{mM} \mathrm{NaCl}, 50 \mathrm{mM}$ imidazole, $\mathrm{pH}$ 7.4) on columns. Proteins were eluted from the resin using the elution buffer (20 $\mathrm{mM}$ sodium phosphate, $500 \mathrm{mM} \mathrm{NaCl}, 500 \mathrm{mM}$ imidazole, $\mathrm{pH}$ 7.4). Subsequently, recombinant G-CSFa1 and G-CSFb1 were purified with gel-filtration to further clarify and simultaneously to determine their molecular weight under a native condition. Gel-filtration was performed using a Sephacryl S-100 column (HR 16/160, GE Healthcare) and the proteins were eluted with $20 \mathrm{mM}$ sodium phosphate buffer containing $300 \mathrm{mM} \mathrm{NaCl}$, pH 7.4 in $0.5 \mathrm{~mL} / \mathrm{min}$ flow rate. The purified proteins were then passed through Pierce High Capacity Endotoxin Removal Columns (Thermo Fisher Scientific) to remove potential traces of endotoxin, buffer exchanged to $1 \mathrm{x}$ PBS with $0.05 \%$ BSA, filter sterilized $(0.22 \mathrm{~mm})$ and stored at $4^{\circ} \mathrm{C}$ or $-80^{\circ} \mathrm{C}$ until use. Residual endotoxin was checked to be less than $5 \mathrm{pg} / \mathrm{mL}$ according to a Limulus ES-II Single Test (Wako, Osaka, Japan). A Bradford assay (Thermo Fisher Scientific) was performed according to manufactures' directions to determine protein concentration.

Recombinant carp erythropoietin (EPO), kit ligand A (KITLA) and thrombopoietin (TPO) were produced and purified as described previously [28, 29].

\section{Cell proliferation assay}

Freshly isolated carp head and trunk kidney leukocytes from 4 individuals were adjusted to a concentration of $4-8 \times 10^{5}$ cells $/ \mathrm{mL}$ in E-RDF20/2.5 medium. Fifty $\mu \mathrm{L}$ of this cell suspension was added to each well of a 96-well plate to which 50 $\mu \mathrm{L}$ of treatment in E-RDF20/2.5 medium was added. Treatments consisted of the 
E-RDF medium (negative control), 25\% cell conditioned medium (CCM) derived from the carp kidney leukocyte culture in which macrophages develop (positive control, [30]), a combination of $100 \mathrm{ng} / \mathrm{mL}$ TPO and $100 \mathrm{ng} / \mathrm{mL}$ KITLA (positive control), recombinant G-CSFa1 or G-CSFb1 at final concentrations of 500, 100, 20, 4, $0.8,0.16 \mathrm{ng} / \mathrm{mL}$ and heat-inactivated $\left(98^{\circ} \mathrm{C}\right.$ for $\left.30 \mathrm{~min}\right)$ recombinant $\mathrm{G}-\mathrm{CSFa} 1$ and G-CSFb1. Cell proliferation was determined using the colorimetric MTT assay (Nacalai Tesque, Kyoto, Japan) which was first shown to provide comparable data for different leukocyte cell types (data not shown). Briefly, $10 \mu \mathrm{L}$ of MTT reagent was added to each well and plates were incubated at $30^{\circ} \mathrm{C}$ for $5 \mathrm{~h}$ to develop a coloration reaction depend on live cell number. One hundred $\mu \mathrm{L}$ of solubilization solution (acid-isopropanol) was then added to each well and plates were sealed and kept at $30^{\circ} \mathrm{C}$ for $12 \mathrm{~h}$. Cell proliferation was determined on days $0,3,6$ and 9 , and plates were read at absorbance of $570 \mathrm{~nm}$ and $650 \mathrm{~nm}$ as a reference using Multiskan GO microplate reader (Thermo Fisher Scientific). Values obtained at absorbance of 650 $\mathrm{nm}$ from each well were subtracted from values obtained at absorbance of $570 \mathrm{~nm}$ from each well.

\section{RT-qPCR analysis of gene expression in cells treated with recombinant G-CSFa1 and/ or G-CSFb1}

Freshly isolated carp kidney leukocytes were seeded into 24-well plates in $0.5 \mathrm{~mL}$ of E-RDF20/2.5 at a concentration of $4 \times 10^{5}$ cells $/ \mathrm{mL}$. Cells were either treated with the medium (untreated control), recombinant G-CSFa1 (100 ng/mL final concentration), recombinant G-CSFb1 (100 ng/mL final concentration) or the combination of $100 \mathrm{ng} / \mathrm{mL} \mathrm{G-CSFa} 1$ and $100 \mathrm{ng} / \mathrm{mL} \mathrm{G-CSFb} 1$ in the E-RDF20/2.5 for $12 \mathrm{~h}$ and 4 days at $30{ }^{\circ} \mathrm{C}$. Following incubation, cells were collected, total RNA was isolated using the NucleoSpin RNA kit (Macherey-Nagel, Düren, Germany) and reverse transcribed into cDNA using the Omniscript RT kit (Qiagen) according to manufacturer's instructions. Quantitative PCR was performed for carp transcription factors known to be involved in early hematopoiesis (gata2) [31], myelopoiesis (pu.1, cebpa and irf8) [32-35], erythropoiesis (gata1) [36] and lymphopoiesis (gata3 and pax5) [37, 38] and myeloid cell markers (gcsfr1, gcsfr2, csf1r and mpx) [22, 35] using a Thermal Cycler Dice Real Time System II (Takara). Beta-actin ( $\beta$-actin) was employed as an endogenous control. Quantitative PCR cycling conditions were $95^{\circ} \mathrm{C}$ for $30 \mathrm{sec}$ followed by 40 cycles of $95^{\circ} \mathrm{C}$ for $5 \mathrm{sec}$ and $60^{\circ} \mathrm{C}$ for $30 \mathrm{sec}$. Data were analyzed using the Thermal Cycler Dice Real Time System software (Takara) and is represented as the average of the four fish $(n=4)$ with standard deviation. 
Likewise, kidney neutrophils from carp were treated with the medium, G-CSFa1 and $\mathrm{G}-\mathrm{CSFb} 1$ in the E-RDF20/2.5 for $6 \mathrm{~h}$ at $30^{\circ} \mathrm{C}$, RNA isolated, and cDNA synthesis as described above. Q-PCR was performed for carp chemokine receptors (cxcr1, cxcr2 and $c x c r 4)[39,40]$ and NADPH oxidase components ( $g p 91^{\text {phox }}, p 22^{\text {phox }}, p 40^{\text {phox }}, p 67^{\text {phox }}$ and $\left.p 47^{\text {phox }}\right)$ [41]. Beta-actin ( $\beta$-actin) was employed as an endogenous control. Quantitative PCR cycling conditions were $95^{\circ} \mathrm{C}$ for $30 \mathrm{sec}$ followed by 40 cycles of $95^{\circ} \mathrm{C}$ for $5 \mathrm{sec}$ and $60^{\circ} \mathrm{C}$ for $30 \mathrm{sec}$. Data were analyzed using the Thermal Cycler Dice Real Time System software (Takara) and is represented as the average of the three fish $(n=3)$ with standard deviation.

\section{Semi-solid colony-forming unit assay}

Freshly isolated leukocytes from carp kidney, spleen or peripheral blood were re-suspended to $2 \times 10^{5}$ cells $/ \mathrm{mL}$ in E-RDF medium containing $40 \% \mathrm{FBS}$ and $5 \%$ carp serum. Colony-forming unit assay using semi-solid media was carried out as previously described [28] with minor modifications. Briefly, a complete methylcellulose medium was prepared by mixing a $2.0 \%$ methylcellulose stock solution with an equal amount of the cell suspension. In some cases (for experiments of colony counting based on the morphology), a complete $0.45 \%$ agarose medium was prepared by mixing an $1.8 \%$ agarose solution; $2 \times$ E-RDF medium; and the cell suspension in the volume ratio of $1: 1: 2$. Then, $2.4 \mathrm{~mL}$ of the cell suspension/ complete semi-solid medium was added to a $5 \mathrm{~mL}$ tube with a $2.5 \mathrm{~mL}$ syringe and 16-gauge blunt-end needle, along with cytokines or PBS. Tubes were tightly capped and the solution gently mixed. One milliliter of the solution (in duplicate) was aliquoted onto a solid E-RDF medium containing $0.45 \%$ agarose (Lonza), $20 \%$ FBS and $2.5 \%$ carp serum in a $35 \mathrm{~mm}$ dish or a 6-well plate. Dishes and Plates were incubated at $30{ }^{\circ} \mathrm{C}$ with an additional $5 \% \mathrm{CO}_{2}$ atmosphere and $100 \%$ humidity for 7-13 days, followed by examination for colony formation.

The number of progenitor cells in each organ was estimated according to the formula described below.

No. of progenitors $=($ Total No. of leukocytes from each organ $) \times($ No. of colonies forming per plate) / (No. of leukocytes plated) 


\section{Characterization of colony cells}

Cell colonies formed in the methylcellulose media were aspirated by micropipette under a microscope and characterized by morphology, cytochemistry and RT-PCR analyses as previously described [28]. In short, the colony cells were re-suspended in MEM, and cyto-centrifuged with a Cytospin (Shandon). Slides were dried, fixed and stained with May-Grünwald Giemsa (MGG, Wako Pure Chemicals, Osaka, Japan) or Peroxidase stain based on the DAB oxidization. For RT-PCR analyses, total RNAs were extracted from each colony cell type using RNeasy Micro Kit (Qiagen) and cDNA was synthesized using Omniscript RT kit (Qiagen). Expression of hematopoietic marker genes was analyzed by PCR using carp specific primers and EmeraldAmp PCR Master Mix (Takara). PCR was conducted as follows: one cycle of $94{ }^{\circ} \mathrm{C}$ for $1 \mathrm{~min}$, followed by 23 to 38 cycles of denaturation at $98^{\circ} \mathrm{C}$ for $30 \mathrm{sec}$, annealing at $58{ }^{\circ} \mathrm{C}$ for $30 \mathrm{sec}$ and elongation at $72^{\circ} \mathrm{C}$ for $30 \mathrm{sec}$. Colonies treated with recombinant carp erythropoietin (EPO) was utilized for the control group of the erythroid lineage.

\section{Neutrophil chemotactic response to recombinant carp G-CSFa1 and G-CSFb1}

Neutrophils obtained from carp trunk kidney were washed twice in MEM and adjusted to a final concentration of $1 \times 10^{6}$ cells $/ \mathrm{mL}$. The chemotaxis assay was performed using blind well chemotaxis chambers (Neuro Probe, Inc.). Two hundred microliters of different concentrations of recombinant carp G-CSFa1 or G-CSFb1 (1, 10 and $100 \mathrm{ng} / \mathrm{mL}$, final concentrations) in the serum free MEM were added to the bottom well of the chemotaxis chambers, and the bottom chamber was separated from the top chamber using $5 \mathrm{~mm}$ pore size polycarbonate membrane filters (Neuro Probe, Inc.). To the top chamber, $200 \mu \mathrm{L}$ of neutrophil suspension was added. Negative controls consisted of medium alone and the positive control was $10 \mathrm{ng} / \mathrm{mL}$ of fMLP (Sigma-Aldrich). The chemokinesis control consisted of $100 \mathrm{ng} /$ $\mathrm{mL}$ of G-CSFa1 or $100 \mathrm{ng} / \mathrm{mL}$ of G-CSFb1 in both the upper and lower chambers of the chemotaxis apparatus.

The incubation period was $1 \mathrm{~h}$ after which the cell suspensions were carefully aspirated from the top chamber and the filters removed and applied bottom side up on a slide glass. Filters were stained with MGG. Chemotactic activity was determined by counting the total number of cells found on the underside of the polycarbonate filters in 20 random fields of view (40× magnification). Technical duplicates were conducted for all treatments ( $n=4$, biological replicates). 


\section{Respiratory burst assay}

Respiratory burst assay was performed as previously described [42] with minor modifications. The neutrophils harvested from carp kidney were re-suspended in E-RDF20/2.5 medium at a concentration of $2.5 \times 10^{6}$ cells $/ \mathrm{mL}$. Four hundred microliters of the cell suspension was added to each $1.5 \mathrm{~mL}$ tubes, and cells were treated or untreated with recombinant carp G-CSFa1 (100 ng/mL) or G-CSFb1 (100 $\mathrm{ng} / \mathrm{mL}$ ) at $25^{\circ} \mathrm{C}$ for $6 \mathrm{~h}$. Following the incubation, DHR123 (Molecular Probes) was added to the cells at a final concentration of $10 \mu \mathrm{M}$ and incubated for $5 \mathrm{~min}$ to allow the cells to take up the DHR123. PMA (Abcam, Cambridge, UK) was then added at a final concentration of $100 \mathrm{ng} / \mathrm{ml}$. The cells were further incubated for $30 \mathrm{~min}$ to allow oxidation of the DHR. All samples were appropriately staggered with respect to timing to accommodate the transient state of oxidized DHR fluorescence. Live cells were gated according to the forward scatter and side scatter parameters. DHR fluorescence was detected in the FITC channel, and the mean values of the FITC fluorescence in neutrophils were normalized to untreated controls.

\section{In vivo effects of G-CSFa1 and G-CSFb1}

Carp were bled $200 \mu \mathrm{L}$ from the caudal vein using a heparinized syringe 2 days before administration of G-CSF paralogs and blood was centrifuged in a capillary glass tube at $1500 \times g$ for $5 \mathrm{~min}$. Leukocytes on top of the erythrocyte layer were obtained, treated with the $1 \times$ red blood cell lysis buffer, washed twice with Hanks' balanced salt solution and then used to measure the ratio of neutrophils per total number of leukocytes by flow cytometry analysis, based on forward scatter versus side scatter parameters. Subsequently, carp were injected intraperitoneal (i.p.) with $100 \mathrm{ng} / \mathrm{g}$ body weight of recombinant proteins in $200 \mu \mathrm{L}$ of $1 \times$ PBS, or were injected with $1 \times$ PBS only. After 6,24 and 48 h, $200 \mu \mathrm{L}$ of peripheral blood was collected and analyzed as described above. Three fish for each group were examined.

\section{Statistical analysis}

Raw data of technical replicates were first averaged per individual before statistical analysis was performed. Statistical analysis was performed after log-transformation of datasets that were not normally distributed. Subsequently, normality was assumed and statistical significance was tested using an un-paired Student's $t$ test (independent observations, Figure 4.3) for one-to-one comparisons and a (repeated-measures) one-way analysis of variance (ANOVA) followed by a Dunnet's post-hoc test (in Figures 4.4, 4.5A - E, 4.7 and 4.8). Or, Tukey's HSD (Figure 4.2) 
and Dunnet's T3 (in case of unequal variances) (Figure 4.2) were used for multiple comparisons. A two-way ANOVA was performed followed by Tukey's post-hoc test for multiple comparisons shown in Figure 4.9 and Figure 4.10. Prism 7 software (GraphPad Software, La Jolla, CA, USA) was used. In absence of sphericity, the Geisser-Greenhouse correction was applied. A value of $p \leqslant 0.05$ was accepted as significant.

\section{Results}

\section{Identification of four carp G-CSF paralogs}

The presence of four G-CSF paralogs in the genome of common carp was expected as common carp has undergone a WGD event compared to the zebrafish [21], in which two G-CSF paralogs are already present. Referring to carp genome and transcriptome databases (project no. PRJEB7241 and PRJNA73579) at the National Center for Biotechnology Information (NCBI), four putative loci encoding carp G-CSF homologs were found next to conserved genes PSMD3, MED24 or LRRC3 (Figure 4.1A). Synteny of each paralog is highly conserved with either the zebrafish G-CSFa locus on chromosome 12 or the GCSFb locus on chromosome 19.

The complete open reading frames (672, 675, 588 and $582 \mathrm{bp}$ ) of four carp paralogs' cDNA transcripts respectively encoding 224, 225, 196 and 194 amino acids with 5 exons were obtained (Figure 4.1B and Supplementary figure S4.1A-D). Despite quite low sequence identity and similarity (Supplementary table S4.1), carp G-CSF paralogs share a similar predicted structure and one of the copies (G-CSFa1) was predicted to have an additional helical region from Ser ${ }^{160}$ to Ser $^{164}$ which is acidic amino acid residue (Asp and Glu)-abundant (Figure 4.1B and Supplementary figure S4.2). Carp G-CSF paralogs all possess the consensus domain of Pfam IL6/GCSF/ MGF protein family, whereas the four cysteine residues involved in two disulfide bonds are not conserved. Carp G-CSF copies also share conserved acidic amino acids involved in major ligand-receptor binding demonstrated in human G-CSF, while there is no acidic amino acid residue near the $\alpha$-helix $E$ in carp G-CSFa2 (Figure 4.1B). Phylogenetic analysis revealed that all the G-CSFs were found to form a single evolutionary clade outside a related cytokine interleukin-6, suggesting that the G-CSFs are orthologous. Taking into account there may be G-CSF paralogs present in the teleost species shown that have not yet been reported, each of the four carp paralogs did cluster with either teleost G-CSFa or G-CSFb paralogs 
(Figure 4.1C). Hence, based on clustering and the conserved synteny, we named the four carp G-CSF paralogs as G-CSFa1, G-CSFa2, and G-CSFb1, G-CSFb2.

\section{Carp g-csf paralogs show differential expression in immune tissues and cells}

Assessment of basal $g$-csfa1 expression in tissues from healthy carp revealed generally very low expression of $g$-csfa 1 in most tissues, with significantly higher gene expression in spleen, muscle and gill (Figure 4.2). G-csfa2 was significantly higher expressed in spleen than in gill, brain, thymus, trunk-kidney and headkidney (Figure 4.2). Basal expression levels of $g$-csfb1 and $g$-csfb2 were generally low or non-detectable in most carp tissues examined (Figure 4.2).

At basal levels, g-csfa expression is markedly higher than g-csfb expression in all immune cells examined. Strikingly, all $g$-csf paralogs were highest expressed at basal level in macrophages, indicating these cells as the major producers of G-CSF, comparable to mammalian macrophages. Within macrophages, g-csfa1 and g-csfb1 were significantly higher expressed compared to their respective counterparts (Figure 4.3A and B). Remarkably, a clear expression of $g$-csfa 1 was observed also in thrombocytes (Figure 4.3A).

In order to determine induction of the different G-CSF paralogs upon antigenic stimulation, we investigated expression levels in freshly isolated kidney leukocytes and head kidney-derived macrophages following the stimulation with LPS, ConA, PMA and poly I:C (only freshly isolated kidney leukocytes). In freshly isolated kidney leukocytes, all paralogs were highly up-regulated after stimulation with LPS and the combination of ConA/PMA at 3 and 6 hours but not after stimulation with Poly I:C (Supplementary figure S4.3). Likewise, in the cultured macrophages gene expressions of the four paralogs were clearly enhanced by LPS and PMA stimulations (Figure 4.4). Despite non-detectable $g$ - $c s f b 1$ transcripts, its gene expression was induced with LPS stimulation. Interestingly, in macrophages both g-csfa 1 and $g$-csfa2 are relatively high expressed at basal level (Figure 4.3) and appear to show a relatively small increase upon stimulation with LPS (Figure 4.4), whereas $g$-csfb1 and $g$-csfb2, which are relatively low or non-detectable at basal level (Figure 4.3), show a large increase in gene expression after LPS stimulation (Figure 4.4). We could also show that expression in macrophages of interleukin-1 beta, which is a pro-inflammatory cytokine, was significantly up-regulated after LPS stimulation (Figure 4.4). 
A

Human Chr. 17

Chicken
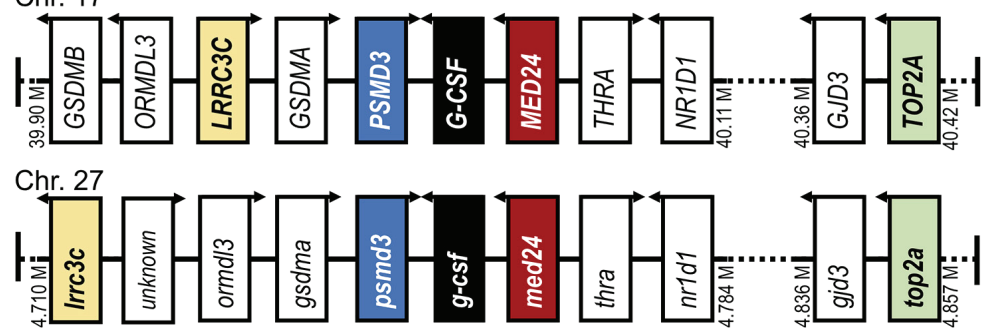

Zebrafish Chr. 12
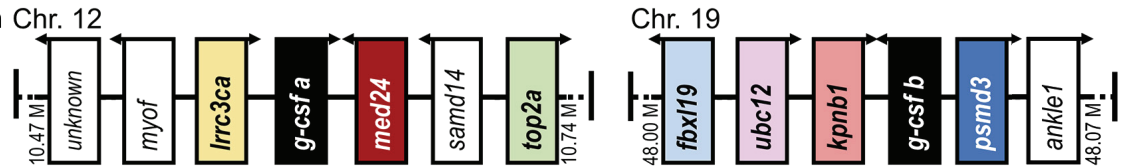

Carp

LHQP01022752.1

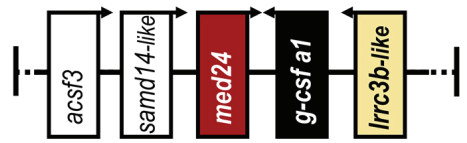

LHQP01012478.1

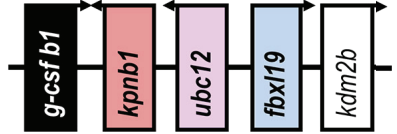

LHQP01015831.1

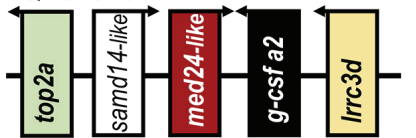

LHQP01028996.1

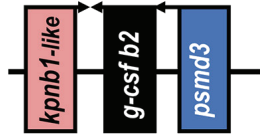

B

Cc G-CSFa1

Cc G-CSFa2

Dr G-CSFa

Cc G-CSFb1

Cc G-CSFb2

Dr G-CSFb

$X 1$ G-CSF

Mm G-CSF

HS G-CSE

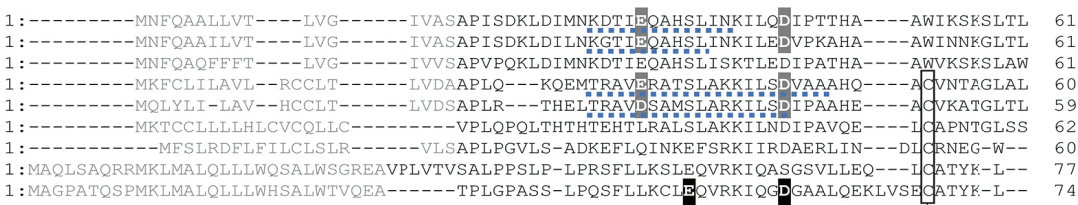

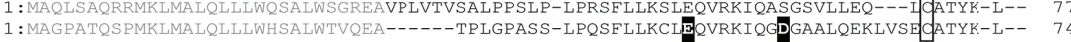

\section{at $000000000000 \mathrm{y}$ ss}

Cc G-CSFal

Cc G-CSFa2

Dr G-CSFa

Cc G-CSFb1

Cc G-CSFb2

Dr G-CSFb

$X \perp$ G-CSF

Mm G-CSF

Hs G-CSE

62 : GD S KARL D. 62 : GDNTIRLQLNYLKS--VIPSAPVLQNISNISSMETCLADMVKGLQLHLNLLNEIIK--KLAQTDOVNVLKNEIDELRSLIKKLO 144 61 :SSEA--GHL WYFLSDLGI PAP PVLK--SEDLSMDVSLSRIVAGLDLHRDILQDIRER--SSSTEELSLLIADITDLSARVHOMO, 141 60 :SS

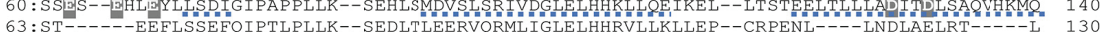

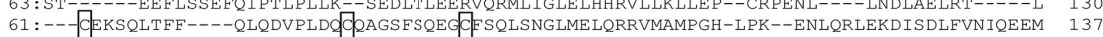
78 :---CHPEELVLLGHS LG I PKASLSGCSSQALQQTOCLSQLHSGLCLYQGL LQALSGI-S PALAPT LDLLQLDVANFATT IWQQM 160

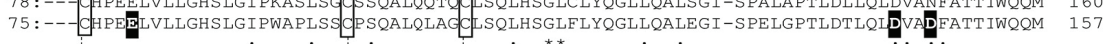

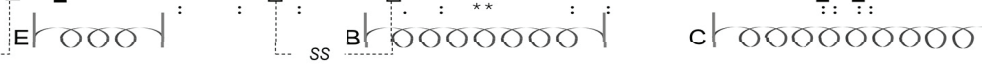

CC G-CSFa CC G-CSFa2 Dr G-CSEa CC G-CSFb1

$\mathrm{CC} \mathrm{G}-\mathrm{CSFb} 2$

$\mathrm{Dr} \mathrm{G}-\mathrm{CSFb}$

$X]$ G-CSE

$M m$ G-CSE

Hs G-CSE

149 : NQAGFDPSKQASDEESQTPEHDLAKRLTNEYLTOVAAHLTLOQLOPDFCDILRSIHSM-----TSSLAENPNTVQLCVNKAGL 223 145:KQAGFDPSNHAKDEQSQTLVHDLHKHLTTEFMIOVAAHLTLQOLQDFSCDVLRSFLSIRRMTSNMSMAENPKTVQLCINAAGL 224

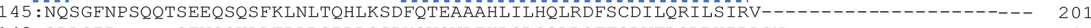

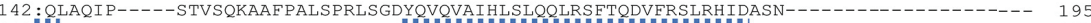
141:

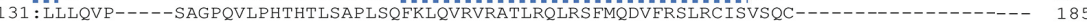
138 : EAQGITAQNS--------PSQTSLPTSTSTFHQKATMFLILSDLADFMRTLHSLLA-------------------- 182 161 : ENLGVAPTVQ-------PTQSAMPAFTSAFQRRAGGVLAISYLQGFLETARLALHHLA----------

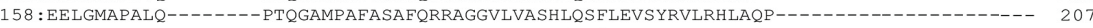
cot ot 00000000000 l

Figure 4.1 Granulocyte colony-stimulating factor paralogs of common carp. Figure legend on p.132 


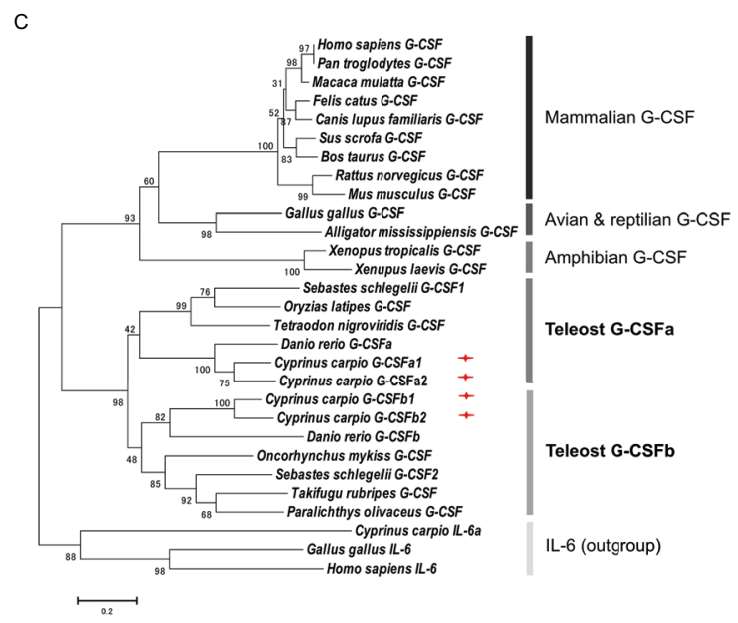

Figure 4.1 Granulocyte colonystimulating factor paralogs of common carp. A) Synteny analysis of G-CSF of human, chicken, zebrafish, and carp. The G-CSF locations in human, chicken, and zebrafish are obtained from respective genome resources at NCBI and carp G-CSF locations are obtained from carp genome and transcriptome projects PRJEB7241 and PRJNA73579 at NCBI. B) Alignment of vertebrate granulocyte colony-stimulating factor (G-CSF) amino acid sequences. Complete protein sequences of carp $C c \mathrm{G}$-CSFa1 (Cyprinus carpio, MG882495), carp Cc G-CSFa2 (MG882496), zebrafish $\mathrm{Dr}$ G-CSFa (Danio rerio, NP_001138714), carp Cc G-CSFb1 (MG882497), carp Cc G-CSFb2 (MG882498), zebrafish Dr G-CSFb (NP_001137226), african clawed frog Xl G-CSF (Xenopus laevis, Scaffold13265:3008399-3011722), mouse Mm G-CSF (Mus musculus, NP_034101), and human Hs G-CSF (Homo sapiens, NP_000750) were aligned using Clustal Omega. Signal sequences are shown with gray letters and conserved cysteine residues are boxed. In the human G-CSF, helices (A to E) are denoted with coils, and Glutamic acid (E) and Aspartic acid (D) residues representing major interfaces with G-CSF receptor are highlighted in black. In the carp G-CSFs, predicted alpha helical regions are underlined with a broken line, modeled on the structure of human G-CSF, and residues expected to interact with the receptor are highlighted in gray. Amino acids that are conserved in all sequences are denoted with an asterisk (*), strong similarity with a colon (:), and weak similarity with a period (.). C) Phylogenetic analysis of mammalian, avian, reptilian, amphibian and teleost fish G-CSF proteins. Phylogenetic analysis included carp G-CSFa1 (C. carpio, MG882495); carp G-CSFa2 (MG882496); carp G-CSFb1 (MG882497); carp G-CSFb2 (MG882498); zebrafish G-CSFa (D. rerio, NP_001138714); zebrafish G-CSFb (NP_001137226); rockfish G-CSF1 (S. schlegelii, BAH56611); rockfish G-CSF2 (BAH56612); medaka G-CSF (O. latipes, XP_004080425); green-spotted puffer G-CSF (T. nigroviridis, CAG04394); rainbow trout G-CSF (O. mykiss, CAQ42965); fugu G-CSF (T. rubripes, XP_003965085); flounder G-CSF (P. olivaceus, BAE16320); african clawed frog G-CSF (X. laevis, Scaffold13265:3008399-3011722); tropical clawed frog G-CSF (X. tropicalis, XP_002940261); alligator G-CSF (A. mississippiensis, XP_006270858); chicken G-CSF (G. gallus, NP_990610); human G-CSF (H. sapiens, NP_000750); chimpanzee G-CSF (P. troglodytes, XP_009430519); rhesus monkey G-CSF (M. mulatta, XP_001095097); cat G-CSF (F. catus, NP_001009227); dog G-CSF (C. lupus familiaris, XP_005624600); pig G-CSF (S. scrofa, XP_005653977); cattle G-CSF (B. taurus, NP_776453); rat G-CSF (R. norvegicus, NP_058800); mouse G-CSF (M. musculus, NP_034101); and outgroup including carp interleukin-6a (IL-6a, AGR82313); chicken IL-6 (NP_989959); and human IL-6 (NP_000591). Phylogenetic analysis was conducted using the neighbor joining method in MEGA version 6 and bootstrapped 1,000 times with bootstrap values expressed as percentages.

\section{Recombinant carp G-CSFa1 and G-CSFb1 are monomeric forms}

Based on expression levels in macrophages and the clear induction in stimulated macrophages, we chose to express two copies, G-CSFa1 and G-CSFb1, to investigate their function. Recombinant G-CSFa1 and G-CSFb1 purified using Ni-affinity chromatography were passed through a gel filtration column under a non- 
denaturing condition to calculate their molecular weights. As a result, the molecular weights of G-CSFa1 and G-CSFb1 were estimated to 25,275 and 22,355, similar to the deduced values based on their primary structures and similar to the result of SDS-PAGE under the denaturing condition, indicating that both recombinants form monomers (Supplementary figure S4.4).

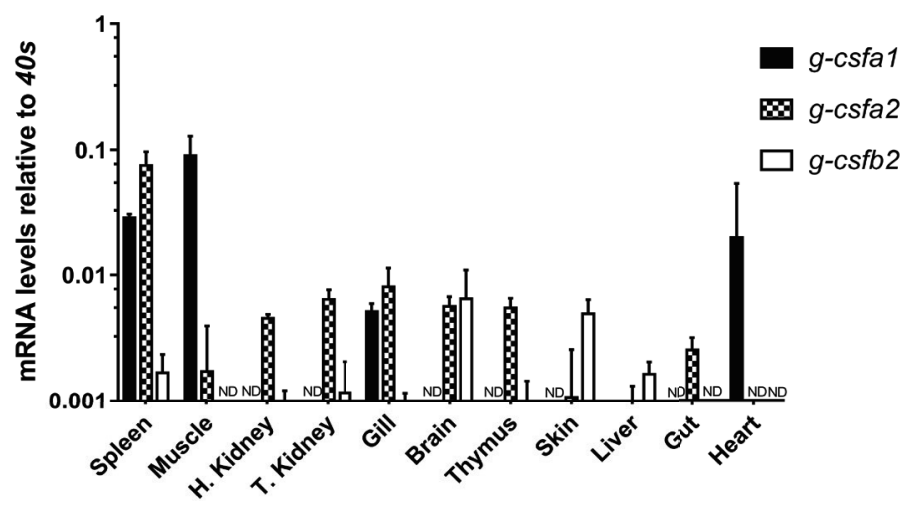

Figure 4.2 Quantitative mRNA expression analysis of carp $g$-csf paralogs in carp tissues. Basal gene expression of carp G-CSF paralogs in spleen, muscle, head kidney, trunk kidney, gill, brain, thymus, skin, liver, gut and heart. Basal expression levels were determined relative to the s11 protein of the $40 \mathrm{~s}$ subunit (40s) as a reference gene and are presented as mean + standard deviation

( $n=3$, except thymus, $n=2$ ). $G$ - $c s f b 1$ expression was non-detectable in all tissues examined (ND). Significant differences in expression between tissues were determined using one-way ANOVA followed by Tukey's HSD ( $g$-csfa1 and $g$-csfb2) or Dunnet's T3 post-hoc test for unequal variances ( $g$-csfa2).
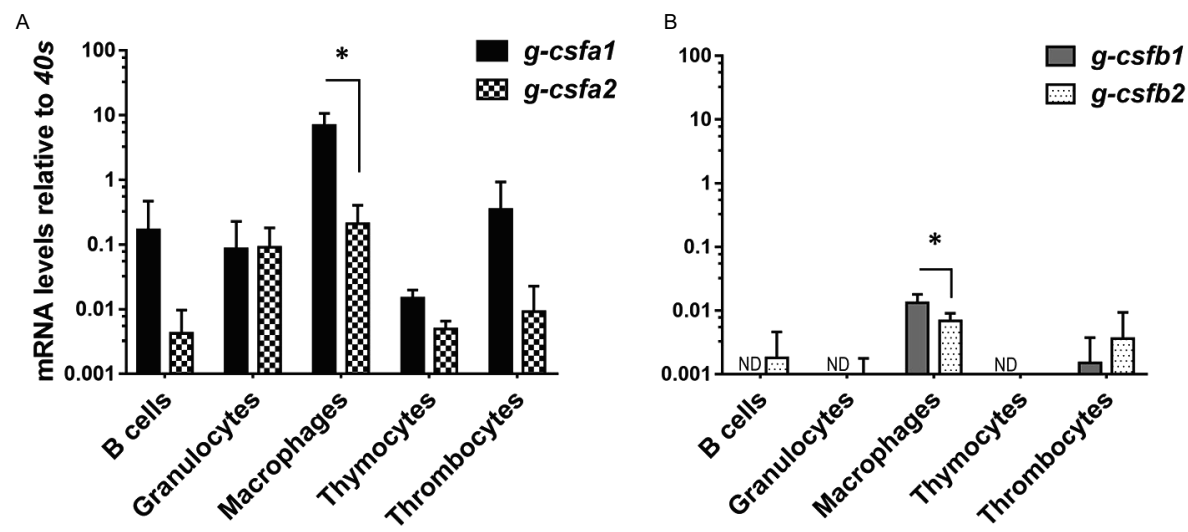

Figure 4.3 Quantitative mRNA expression analysis of carp g-csf paralogs in immune cells. Basal gene expression of carp G-CSF paralogs in sorted cells from healthy carp. Basal expression levels were determined relative to the s11 protein of the 40 s subunit (40s) as a reference gene and presented as mean + standard deviation ( $n=4$ except thymocytes and thrombocytes $n=2)$. Statistical significance within the macrophage group was determined using an un-paired Student's $t$ test. Asterisks (*) denotes significance $(p<0.05)$ between indicated genes. ND indicates "non-detectable". 
Both G-CSFa1 and G-CSFb1 induce proliferation of kidney leukocytes, but only G-CSFb1 induce differentiation of cells along the neutrophil lineage

Carp kidney leukocytes treated with the cell conditioned medium containing macrophage growth factor(s) and recombinant TPO plus KITLA exhibited active proliferation, indicating that there are heterogeneous hematopoietic progenitors in the kidney leukocytes (Figure 4.5A and Supplementary figure S4.5). Treatment of carp kidney leukocytes with $0.8,4,20,100$ and 500 ng/mL of G-CSFa1 induced a dose-dependent proliferative response, with the highest proliferation observed in cells treated with more than $20 \mathrm{ng} / \mathrm{mL}$ of G-CSFa1, whereas heat-inactivated ( $98^{\circ} \mathrm{C}$ for $30 \mathrm{~min}$ ) $\mathrm{G}-\mathrm{CSFa} 1$ had no effect. Likewise, treatment of kidney leukocytes with 4, 20, 100 and $500 \mathrm{ng} / \mathrm{mL}$ of G-CSFb1 induced a dose-dependent proliferative response, with the highest proliferation observed in cells treated with more than $100 \mathrm{ng} / \mathrm{mL}$ of G-CSFb1, whereas heat-inactivated G-CSFb1 had no effect (Figure 4.5A and Supplementary figure S4.5). Furthermore, treatment of kidney leukocytes with a combination of $100 \mathrm{ng} / \mathrm{mL}$ of G-CSFa1 and $100 \mathrm{ng} / \mathrm{mL}$ of G-CSFb1 enhanced the proliferative response compared with those cells treated with G-CSFa1 alone or G-CSFb1 alone (data not shown).
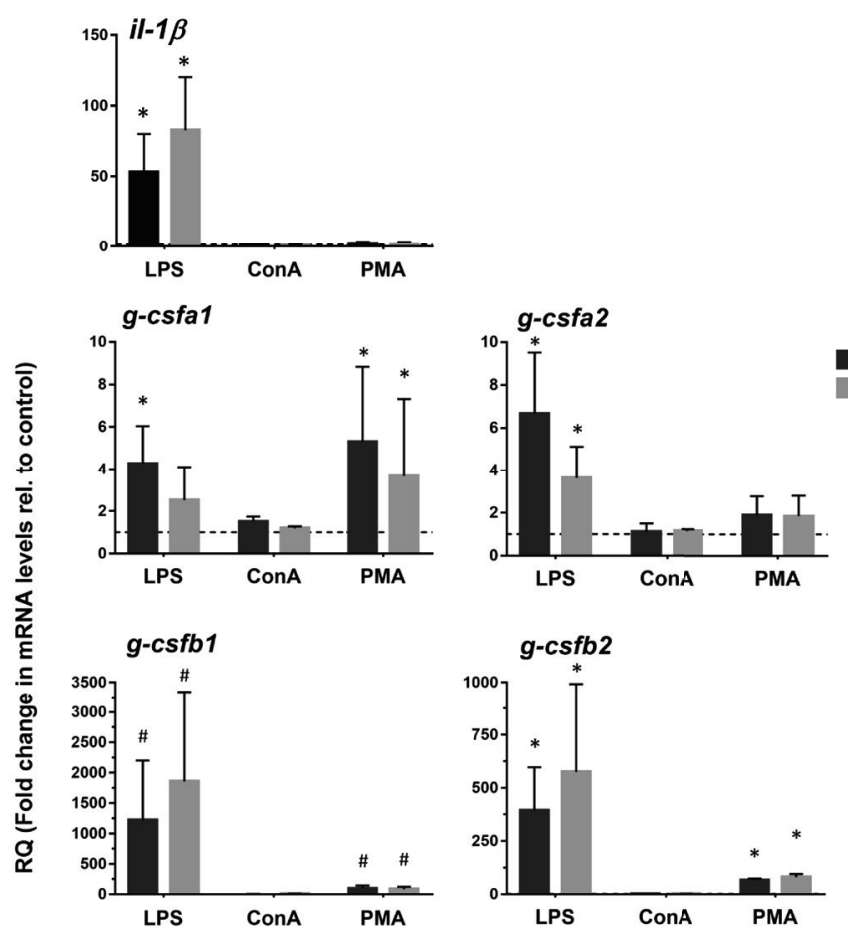

Figure 4.4 Quantitative expression analysis of carp g-csf paralogs in stimulated macrophages. Figure legend on p. 135. 
A lot of growing cells treated with G-CSFa1 adhered onto the plastic and with each other, whereas cells treated with G-CSFb1 exhibited low adhesive property and dispersed (Figure 4.5B). Morphologically, most cells treated with G-CSFa1 for 8 days were blast-like cells, having a basophilic cytoplasm and round to oval nuclei (Figure 4.5C). In contrast, the cells treated with G-CSFb1 for 8 days appeared to be at different developmental stages from myeloblast-like to metamyelocytelike (Figure 4.5C). Most growing cells with each treatment were ascertained to be myeloid cells by staining with TCL-BE8 monoclonal antibody which mainly binds to carp neutrophils [43] (data not shown).

To characterize the roles of G-CSFa1 and G-CSFb1, we examined the gene expressions of transcription factors (TFs) and cell surface markers involved in the development of various cell lineages in carp kidney leukocytes treated with G-CSFa1, G-CSFb1 and a combination of G-CSFa1 and G-CSFb1. The mRNA levels of the TFs involved in myelopoiesis ( $p u .1$, cebp $\alpha$ and irf8), early hematopoiesis (gata2), erythropoiesis (gata1) and lymphopoiesis (gata3 and pax5) in cells treated with or without G-CSFa1, G-CSFb1 and a combination of them for $12 \mathrm{~h}$ were analyzed by quantitative PCR. Kidney cells treated with G-CSFa1 did not undergo any change of TFs mRNA levels. On the other hand, kidney cells treated with G-CSFb1 exhibited a significant up-regulation of cebp $\alpha$ mRNA levels compared to those of the mediumtreated controls (Figure 4.5D), while other TFs tested showed no significant change (data not shown). Cells treated with the combination of G-CSFa1 and G-CSFb1 also showed a moderate up-regulation of cebp $\alpha$ levels compared to those of the controls (Figure 4.5D). Next, we examined whether G-CSFa1 and G-CSFb1 modulate expression of myeloid cytokine receptors and neutrophil-specific myeloperoxidase in carp kidney cells. The mRNA levels of $c s f 1 r, g c s f r 1, g c s f r 2$ and $m p x$ in cells treated with the same treatments for 4 days were analyzed by quantitative PCR. Expression of $c s f 1 r$, which is the macrophage colony-stimulating factor receptor gene, in the kidney cells was unaffected with any treatment examined (Figure 4.5E). Gcsfr1

Figure 4.4 Quantitative expression analysis of carp g-csf paralogs in stimulated macrophages. Gene expression analysis of head kidney-derived macrophages stimulated for 3 or $6 \mathrm{~h}$ with $50 \mu \mathrm{g} / \mathrm{mL}$ lipopolysaccharide (LPS), $10 \mu \mathrm{g} / \mathrm{mL}$ Concanavalin A (ConA) or $1 \mu \mathrm{g} / \mathrm{mL}$ phorbol myristate acetate (PMA). Gene expression was normalized relative to the $s 11$ protein of the 40 s subunit as a reference gene and expressed relative to unstimulated timepoint controls (dashed line at $\mathrm{y}=1$ ). Data are presented as mean + standard deviation $(n=4)$. Significant differences compared to unstimulated timepoint controls were determined using one-way ANOVA followed by Dunnet's post-hoc test, $(p<0.05)$ are denoted by asterisks (*). Non-detectable samples were given an arbitrary value of CT $=32$. Hash mark (\#) indicate significant differences using these arbitrary values. 

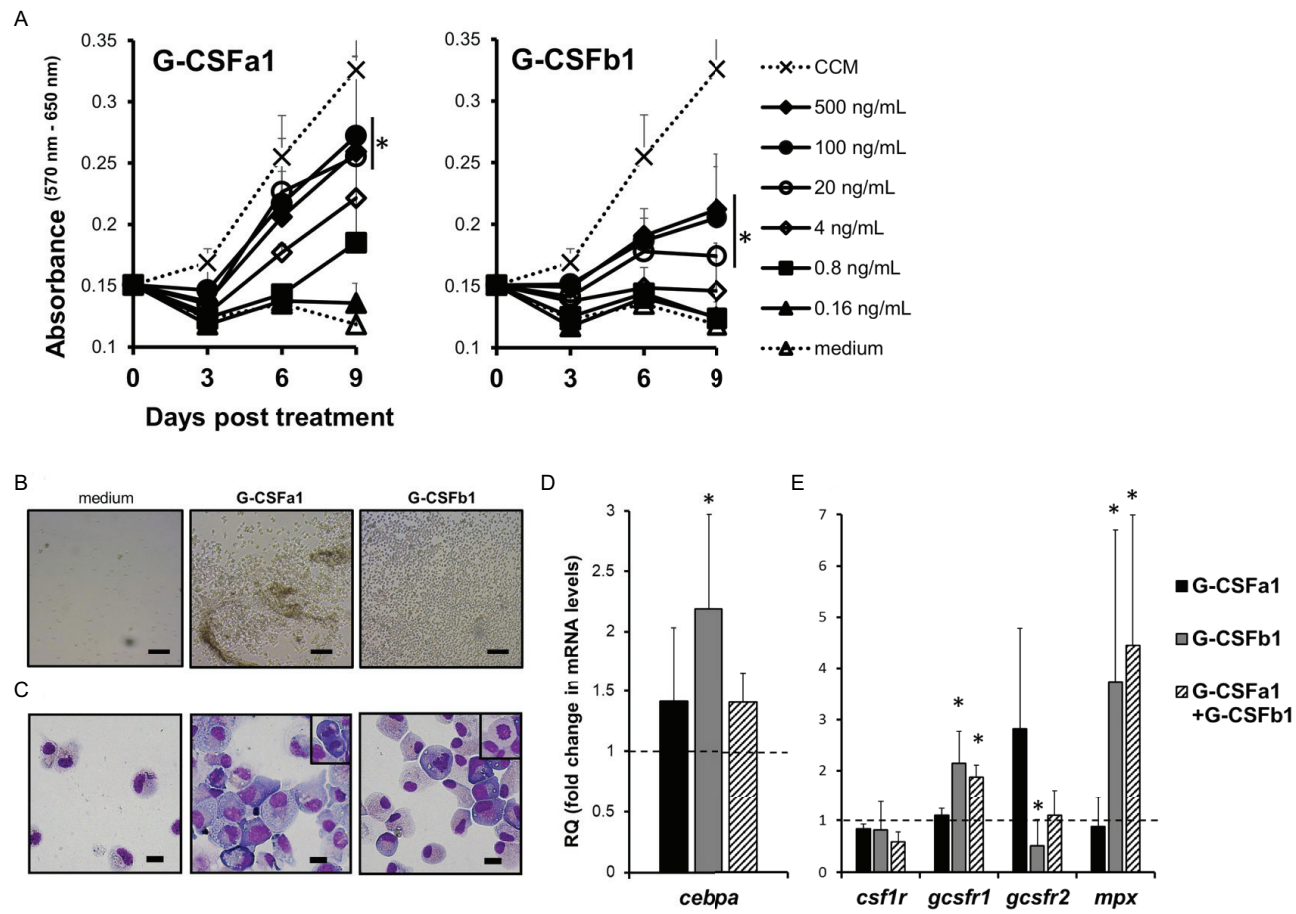

Figure 4.5 Proliferation and differentiation of carp kidney neutrophilic granulocyte-like cells. A) Proliferative response of carp kidney leukocytes (20,000 cells) treated with medium alone, cell conditioned medium (CCM) derived from kidney leukocyte cultures in which macrophages develop, or recombinant carp G-CSF paralogs at different doses. Live cells treated with each stimulus were measured with the MTT assay at day 0, 3, 6 and 9 in the culture. Absorbance values at $650 \mathrm{~nm}$ were subtracted from experimental absorbance values at $570 \mathrm{~nm}$ in each well. Each point in the graphs represents mean + standard deviation $(n=4)$. Significant differences compared to medium-treated controls in day 9 were determined using one-way ANOVA followed by Dunnet's post-hoc test, $(p<0.05)$ are denoted by asterisks (*). B) Photomicrographs of liquid cultures in the absence (medium only) or presence of recombinant G-CSFa1 and G-CSFb1 after 8 days of culture. Scale bars indicate $100 \mu \mathrm{m}$. C) May-Grunwald Giemsa staining of kidney cells after 8 days culture in the absence or presence of G-CSFa1 and G-CSFb1. Mitotic figures were frequently observed (small enclosure). Scale bars indicate $10 \mu \mathrm{m}$. D) Quantitative gene expression analysis of carp transcription factors involved in granulopoiesis (cebp $\alpha$ ) in carp kidney leukocytes treated or untreated with G-CSFa1 $(100 \mathrm{ng} / \mathrm{mL}), \mathrm{G}-\mathrm{CSFb} 1(100 \mathrm{ng} / \mathrm{mL})$ or a combination of G-CSFa1 (100 ng/mL) and G-CSFb1 (100 ng/mL) for $12 \mathrm{~h}$. The mRNA levels were calculated using $\beta$-actin as a reference gene. Data were normalized to the control cells (dashed like at $y=1$ ) and mean + standard deviation is shown $(n=4)$. Significant differences compared to unstimulated controls were determined using one-way ANOVA followed by Dunnet's post-hoc test, $(p<0.05)$ are denoted by asterisks $\left(^{*}\right)$. E) Quantitative gene expression analysis of myeloid cytokine receptors and myeloperoxidase in carp kidney leukocytes treated or untreated with G-CSFa1, G-CSFb1 or a combination of G-CSFa1 and G-CSFb1 for 4 days. The mRNA levels were calculated using $\beta$-actin as a reference gene. Data were normalized to the control cells (dashed line at $\mathrm{y}=1$ ) and mean + standard deviation is shown $(n=4)$. Significant differences compared to unstimulated controls were determined using one-way ANOVA followed by Dunnet's posthoc test, $(p<0.05)$ are denoted by asterisks (*). 
and $m p x$ expression in the kidney leukocytes was up-regulated with the treatment of G-CSFb1 alone and the combination of G-CSFa1 and G-CSFb1, but not with G-CSFa1. On the other hand, gcsfr2 expression in the kidney leukocytes shows a trend towards upregulation with G-CSFa1 treatment but downregulation with G-CSFb1 treatment (Figure 4.5E).

\section{G-CSFb1 stimulates granulocyte colony formation and cooperates with G-CSFa1 to stimulate granulocyte/macrophage colony formation}

In order to further examine the hematopoietic function G-CSFa1 and G-CSFb1 and identify granulocyte progenitor cells, we used an in vitro methylcellulose/agarose colony assay system. As expected, plating of carp kidney leukocytes (100,000 cells) without addition of cytokine resulted in no colony formation (data not shown). In the presence of G-CSF paralogs, overall two types of colonies appeared (Figure 4.6A). Surprisingly, when carp kidney leukocytes were cultured with G-CSFa1 alone, few colony formations were observed at any dose (Figure 4.6B left). On the other hand, in the presence of G-CSFb1, approximately 25 homogeneous colonies were formed after 7 days of the incubation (Figure 4.6B middle). These colonies consisted of uniform small round cells scattered (type 1, Figure 4.6A left). When kidney cells were cultured with a combination of G-CSFa1 and G-CSFb1, morphologically two kinds of colonies were observed. One appeared to be similar to the type 1 colonies formed in the presence of G-CSFb1 alone, the other seemed to consist of roughly agminated cells with distinct sizes and shapes (type 2, Figure 4.6A right). Approximately ten type 1 colonies per 100,000 cells plated were formed at day 5 to 7 in the culture and then gradually disappeared. The peak of type 2 colony formation (about 20 colonies per 100,000 cells plated) was observed after 11 days of cultivation (Figure 4.6B right). Both type 1 and type 2 colony cells consisted of morphologically neutrophil lineage cells at distinct developmental stages, which are myeloperoxidase-positive and -negative (Figure 4.6C and D). To characterize colony types, the expression of lineage-associated marker genes was analyzed. Figure 4.6E shows a typical expression patterns in type 1 and type 2 colonies. Type 1 colonies treated with G-CSFb1 alone or G-CSFa1 plus G-CSFb1 highly expressed g-csfr, cebp $\alpha$ and mpx mRNAs involved in neutrophil development and slightly expressed csf1r which is the macrophage colony-stimulating factor receptor gene, but did not express other genes examined, indicating that type 1 colonies are derived from the progenitor cells corresponding to mammalian granulocyte colony-forming units (CFU-G). Type 2 colonies treated with the combination of 
G-CSFa1 and G-CSFb1 highly transcribed not only neutrophil-specific marker genes but also monocyte/macrophage lineage markers csf1r and irf8, suggesting that type 2 colonies are derived from the progenitors corresponding to mammalian granulocyte/macrophage CFU (CFU-GM) (Figure 4.6E).

A
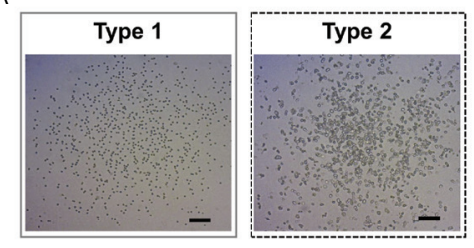

C

C
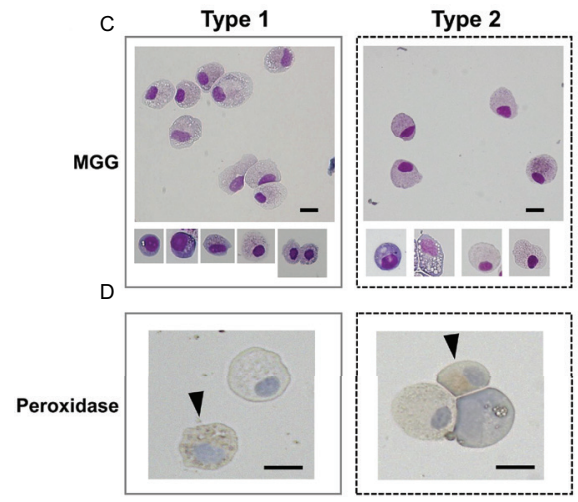

B
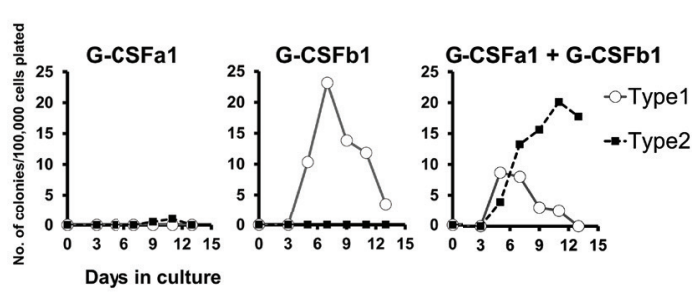

$E$

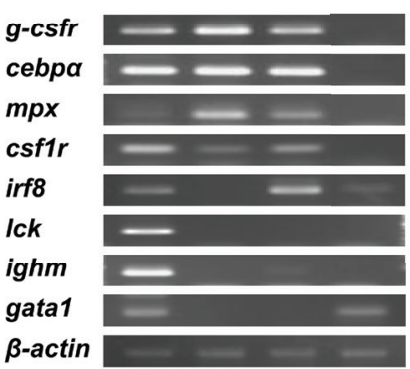

Figure 4.6 Colony formation of kidney cells in response to recombinant carp G-CSFa1 and G-CSFb1. A) Colony-formation of kidney cells in response to G-CSF paralogs. Overall two types of colonies (type 1 and type 2) were observed. Bars indicate $200 \mu \mathrm{m}$. B) Colony counts during semi-solid culture of kidney cells $\left(1 \times 10^{5}\right)$ in the presence of $100 \mathrm{ng} / \mathrm{mL}$ G-CSFa1 alone, $100 \mathrm{ng} / \mathrm{mL} \mathrm{G-CSFb} 1$ alone, or a combination of $100 \mathrm{ng} / \mathrm{mL} \mathrm{G-CSFa} 1$ and $100 \mathrm{ng} / \mathrm{mL} \mathrm{G-CSFb1}$. Each point indicates mean colony counts from 4 individual fish under each condition. Cultures scored every 2 days between 3 to 13 days of incubation. C) May-Grünwald Giemsa (MGG) staining of colony cells (type 1; left and type 2; right). Bars indicate $10 \mu \mathrm{m}$. D) Peroxidase-staining of cells obtained from type 1 colonies (left) and type 2 colonies (right), counterstained with Mayer's Hematoxylin. Arrow heads indicate myeloperoxidase-positive cells. Bars indicate $10 \mu \mathrm{m}$. E) RT-PCR analysis for expression of lineage-associated marker genes in type 1 (lane 1) and type 2 (lane 2) colony cells. cDNA from carp kidney leukocytes was used as a positive control (lane K). cDNA from cells cultured in the presence of $100 \mathrm{ng} / \mathrm{mL}$ carp EPO was utilized for the control group of the erythroid lineage (lane E). 
Granulocyte/macrophage progenitors and granulocyte progenitors are localized in the head kidney and trunk kidney but not in the spleen of carp

To assess the contribution of hematopoietic organs to the neutrophil development in common carp, a myeloid colony forming assay was performed. Leukocytes were harvested from head kidney, trunk kidney, spleen and peripheral blood of adult carp (10 to $15 \mathrm{~cm}$ in length). Approximately $1 \times 10^{5}$ cells were cultured in the methylcellulose/agarose media in the presence or absence of $100 \mathrm{ng} / \mathrm{mL} \mathrm{G-CSFa} 1$ plus $100 \mathrm{ng} / \mathrm{mL} \mathrm{G-CSFb1}$ and colony counts were performed after 6 - 11 days in the culture. PBLs and splenocytes did not form any colonies regardless of addition of cytokine or not. Conversely, cells from head kidney and trunk kidney formed about 25 to 40 colonies of each of type 1 and type 2 in the presence of both G-CSFa1 and G-CSFb1. Total number of CFU-G and CFU-GM in each organ was estimated as table 4.1 .

Table 4.1 The number of type 1 and type 2 colonies formed from 100,000 cells in head kidney, trunk kidney, spleen and PBLs in the semi-solid culture with the combination of $100 \mathrm{ng} / \mathrm{mL}$ G-CSFa1 and $100 \mathrm{ng} / \mathrm{mL}$ G-CSFb1.

\begin{tabular}{|c|c|c|c|c|c|c|c|c|}
\hline & \multicolumn{2}{|c|}{ Head kidney } & \multicolumn{2}{|c|}{ Trunk kidney } & \multicolumn{2}{|c|}{ Spleen } & \multicolumn{2}{|c|}{ PBLs } \\
\hline & $\begin{array}{l}\text { Type } 1 \\
\text { (CFU-G) }\end{array}$ & $\begin{array}{c}\text { Type } 2 \\
\text { (CFU-GM) }\end{array}$ & $\begin{array}{l}\text { Type } 1 \\
\text { (CFU-G) }\end{array}$ & $\begin{array}{c}\text { Type } 2 \\
\text { (CFU-GM) }\end{array}$ & $\begin{array}{l}\text { Type } 1 \\
\text { (CFU-G) }\end{array}$ & $\begin{array}{c}\text { Type } 2 \\
\text { (CFU-GM) }\end{array}$ & $\begin{array}{l}\text { Type } 1 \\
\text { (CFU-G) }\end{array}$ & $\begin{array}{c}\text { Type } 2 \\
\text { (CFU-GM) }\end{array}$ \\
\hline $\begin{array}{l}\text { Mean of colonies formed } \\
\text { in the presence of both } \\
\text { G-CSFa1 and G-CSFb1 } \\
( \pm \text { SEM) }\end{array}$ & $29.1 \pm 4.2$ & $34.5 \pm 4.5$ & $22.9 \pm 3.5$ & $34.0 \pm 3.8$ & 0 & 0 & 0 & 0 \\
\hline $\begin{array}{l}\text { Mean of leukocytes in each } \\
\text { whole organ }\end{array}$ & \multicolumn{2}{|c|}{$9.24 \times 10^{6}$} & \multicolumn{2}{|c|}{$8.94 \times 10^{6}$} & \multicolumn{2}{|c|}{$3.60 \times 10^{5}$} & \multicolumn{2}{|c|}{ ND } \\
\hline $\begin{array}{l}\text { No. of progenitors estimated } \\
( \pm \text { SEM) }\end{array}$ & $\begin{array}{c}2689 \pm \\
388\end{array}$ & $\begin{array}{c}3188 \pm \\
416\end{array}$ & $2047 \pm 313$ & $\begin{array}{c}3039 \pm \\
340\end{array}$ & 0 & 0 & 0 & 0 \\
\hline
\end{tabular}

Data were obtained from duplicate cultures in the presence or absence of both $100 \mathrm{ng} / \mathrm{mL}$ G-CSFa1 and $100 \mathrm{ng} / \mathrm{mL} \mathrm{G-CSFb1}$ and shown only from the culture with the cytokines $(\mathrm{n}=4)$. Type 1 colonies were counted at 7 days of cultivation. Type 2 colonies were counted at 10 days of cultivation. ND, not determined.

\section{G-CSFa1 and G-CSFb1 directly induce a chemotactic response of kidney neutrophils and up-regulates the gene expression of a chemokine receptor cxcr1}

Following the development of neutrophils at the sites of hematopoiesis, the migration and the recruitment of these cells towards the sites of infection or injury is essential for an efficient inflammatory response. We investigated the chemotactic effect of recombinant G-CSFa1 and G-CSFb1 on kidney neutrophils from normal 
adult carp employing a blind-well chemotaxis apparatus (Supplementary figure S4.6). Neutrophils migrated towards fMLP placed in the bottom chamber (Figure 4.7), consistent with previous reports [22]. In the presence of high doses of G-CSFa1 or G-CSFb1, kidney neutrophils migrated towards the sources (Figure 4.7). The chemokinesis controls indicated that neutrophil migration was cytokine-gradient dependent, since the migration of neutrophils was similar to the medium control when the recombinants were placed in both upper and lower chemotaxis chamber (Figure 4.7).

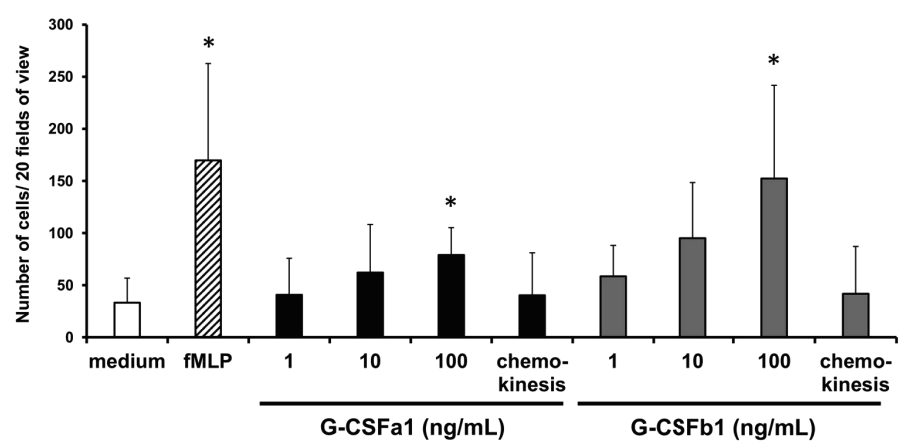

Figure 4.7 Recombinant G-CSFa1 and G-CSFb1 induces chemotactic response of kidney neutrophils. Chemotactic response of kidney neutrophils after one hour of incubation with duplicate filters separating cells and cytokines at the concentrations indicated. Cells were stained with MGG and the total number of cells in 20 random fields of view (40× magnification) was determined. Medium and $10 \mathrm{ng} / \mathrm{mL}$ fMLP served as negative and positive controls respectively. Equal concentrations $(100 \mathrm{ng} / \mathrm{mL})$ of cytokines in the upper and lower chemotaxis chambers served as chemokinesis control. The data represent mean + standard deviation $(n=4)$. Significant differences compared to medium control were determined using one-way ANOVA followed by Dunnet's post-hoc test, $(p<0.05)$ are denoted by asterisks $\left(^{*}\right)$.

To assess the ability of G-CSFa1 and G-CSFb1 to modulate the gene expression of chemokine receptors, kidney neutrophils were treated with medium, G-CSFa1 or G-CSFb1 for $6 \mathrm{~h}$. Teleost CXCR1 and CXCR2 are conserved receptors for interleukin-8 (IL-8, also termed CXCL8) and are important for the regulation of neutrophil recruitment and migration to sites of infection and injury [44, 45]. Cxcr1 mRNA levels in neutrophils treated with G-CSFa1 and G-CSFb1 were significantly up-regulated compared to the medium control, indicating that both enhances a chemotactic sensibility of neutrophils towards chemotactic mediators such as IL-8 (Figure 4.8A). Neither cxcr2 mRNA levels in neutrophils treated with G-CSFa1 nor $\mathrm{G}-\mathrm{CSFb} 1$ were changed compared to the medium control (Figure 4.8A). The mRNA levels of cxcr4 encoding a receptor for stromal cell-derived factor 1 (SDF-1, also termed CXCL12) in neutrophils were not modulated with the treatment of G-CSFa1 and G-CSFb1 (Figure 4.8A). 


\section{G-CSFa1 and G-CSFb1 enhance the respiratory burst capacity in kidney neutrophils through up-regulation of a NADPH oxidase component $\mathrm{p} 47^{\text {phox }}$}

The respiratory burst in neutrophils is the result of the formation of superoxide anions, in a process catalyzed by NADPH-oxidase [46, 47]. Fish NADPH-oxidase components have been shown to have similar modes of activation and functional activities to mammalian counterparts [41, 48]. To assess if the NADPH oxidase is induced by G-CSFa1 and G-CSFb1 treatments, we measured the mRNA levels of NADPH oxidase components ( $g p 91^{\text {phox }}, p 22^{\text {phox }}, p 47^{\text {phox }}, p 67^{\text {phox }}$ and $p 40^{\text {phox }}$ ) in neutrophils treated with G-CSFa1 and G-CSFb1 for $6 \mathrm{~h}$. mRNA levels of $p 47^{\text {phox }}$ in neutrophils treated with G-CSFa1 and G-CSFb1 and $p 40^{\text {phox }}$ in neutrophils treated with G-CSFb1 were significantly increased compared to the medium control. In contrast, mRNA levels of other components were not significantly changed (Figure 4.8B).
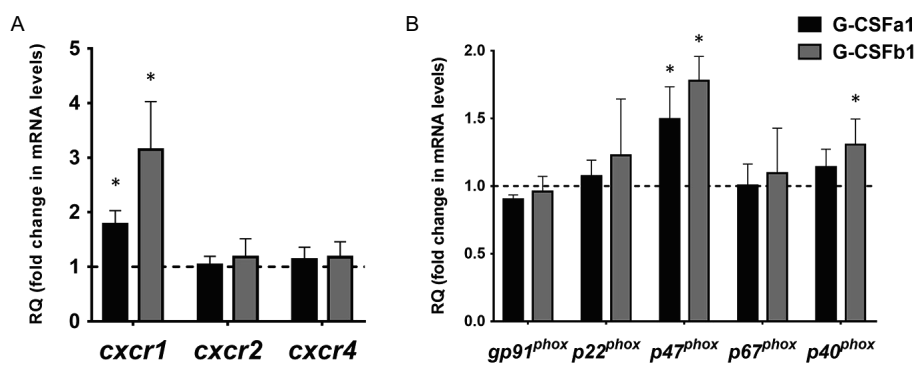

Figure 4.8 Recombinant G-CSFa1 and G-CSFb1 up-regulates cxcr1 and $p 47^{\text {phox }}$ mRNA expression levels in carp kidney neutrophils. Quantitative expression analysis of mRNA levels of chemokine receptors A) and NADPH oxidase components B) in carp kidney neutrophils treated with the medium, $100 \mathrm{ng} / \mathrm{mL}$ G-CSFa1 and $100 \mathrm{ng} / \mathrm{mL}$ G-CSFb1 for $6 \mathrm{~h}$. The mRNA levels were calculated using $\beta$-actin as a reference gene. Data were normalized to the control cells (dashed line at $\mathrm{y}=$ 1 ) and presented as mean + standard deviation $(n=3)$. Significant differences compared to unstimulated controls were determined using one-way ANOVA followed by Dunnet's post-hoc test, $(p<0.05)$ are denoted by asterisks $(*)$.

Furthermore, in order to investigate whether the treatment of carp kidney neutrophils with G-CSFa1 or G-CSFb1 induces their priming to prepare antimicrobial responses, we measured the respiratory burst in PMA-stimulated neutrophils. Neutrophils were pre-treated with the medium, G-CSFa1 or G-CSFb1 for $6 \mathrm{~h}$. Following these treatments, neutrophils were treated with or without PMA in the presence of DHR 123 and then analyzed by flow cytometry. Neither treatment of neutrophils with G-CSFa1 nor G-CSFb1 directly induced the respiratory burst without PMA stimulation (Figure 4.9). Both G-CSFa1 and G-CSFb1 significantly up-regulated the respiratory burst in PMA-stimulated neutrophils compared to 
the medium control, while the enhancement of respiratory burst in neutrophils treated with G-CSFb1 was higher than that of G-CSFa1 treated neutrophils (Figure 4.9 ), which is consistent with the result of the upregulation of $p 47^{\text {phox }}$ enhancement (Figure 4.8B).

\section{In vivo administration of G-CSFa1 and G-CSFb1 increases circulating neutrophils}

Following intraperitoneal (i.p.) injection of PBS and repeated bleeding, the population of peripheral blood neutrophils did not change for $24 \mathrm{~h}$. In contrast, i.p. injection of G-CSFa1 induced a significant increase of peripheral blood neutrophils 6 and $24 \mathrm{~h}$ after injection. Likewise, the population of peripheral blood neutrophils was significantly increased after 6 and $24 \mathrm{~h}$ of G-CSFb1 injection. At $24 \mathrm{~h}, \mathrm{G}-\mathrm{CSFb} 1$ injection had induced a significantly higher circulating number of neutrophils than injection with G-CSFa1 (Figure 4.10). However, at $48 \mathrm{~h}$ after G-CSFa1 injection, neutrophil numbers no longer were higher than those of unhandled or PBSinjected fish, probably due to the repeated bleedings affecting the peripheral blood neutrophil population of the control groups (Supplementary figure S4.7).

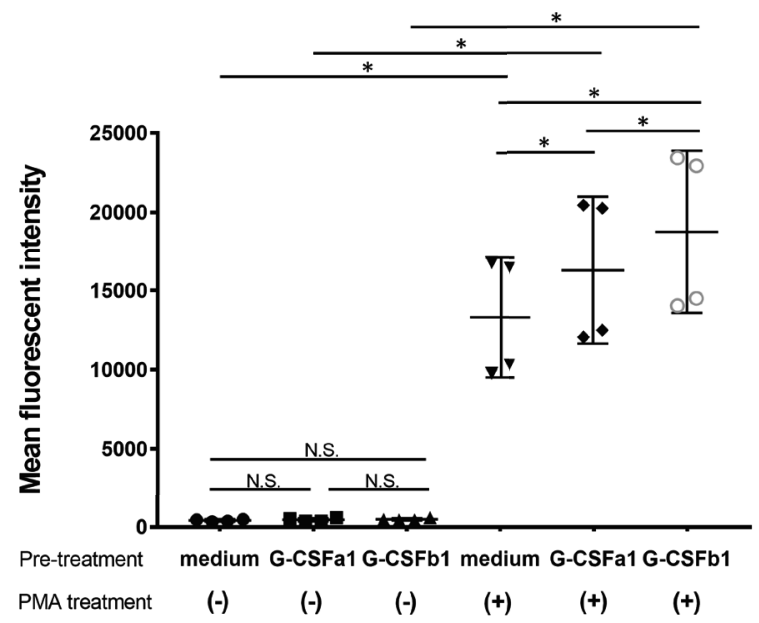

Figure 4.9 Recombinant G-CSFa1 and G-CSFb1 induces increased respiratory burst capability. Respiratory burst capability of kidney neutrophils after pre-treatment with the medium, $100 \mathrm{ng} / \mathrm{mL}$ G-CSFa1 or $100 \mathrm{ng} / \mathrm{mL} \mathrm{G}-\mathrm{CSFb} 1$ for $6 \mathrm{~h}$ and subsequently treated with or without $100 \mathrm{ng} / \mathrm{mL}$ PMA for $30 \mathrm{~min}$ in the presence of DHR123. Mean of DHR123 fluorescence intensity (MFI) in gated neutrophil population was measured by flow cytometry. Data points are presented as mean values of individuals and error bars show standard deviation. Kidney neutrophils were obtained from four fish. Significant differences compared to every other group with two factors of G-CSF pre-treatment and PMA treatment were determined using two-way ANOVA followed by Tukey's post-hoc test, $(p<0.05)$ are denoted by asterisks (*). N.S. represents 'not significant'. 


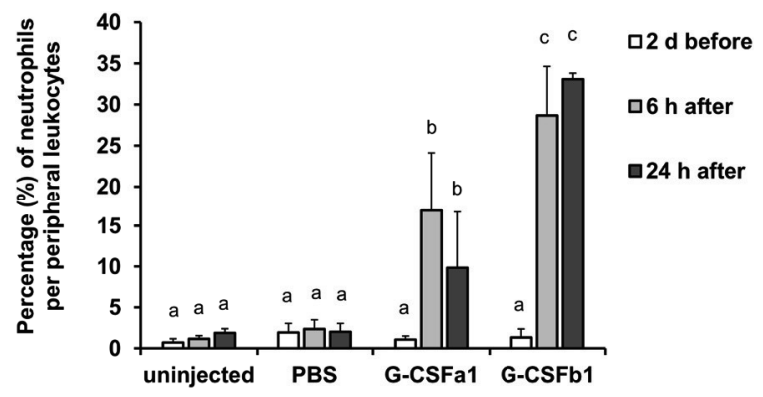

Figure 4.10 Administration of G-CSFa1 and G-CSFb1 to carp increases circulating blood neutrophil population. Peripheral blood leukocytes were collected from carp 2 days before and 6 and 24 hours after intraperitoneal injection of 1 xPBS, recombinant G-CSFa 1 and G-CSFb1 or of unhandled carp and analyzed by flow cytometry. Percentage of gated neutrophil population per live peripheral blood leukocytes was measured. Three fish

for each group were used and data are presented as mean + standard deviation. Significant differences compared to other treatment groups and other time points were determined using two-way ANOVA followed by Tukey's post-hoc test, $(p<0.05)$ are denoted by different letters.

\section{Discussion}

Here we cloned and functionally characterized carp G-CSF. All four carp g-csf genes contain five exons separated by four introns, retaining the gene structure found in human and mouse G-CSF as well as G-CSF of other teleost species [13]. Carp and human G-CSF molecules share a similar structure of a signal peptide and a fourplus-one helical Pfam IL6/GCSF/MGF domain. All teleost fish G-CSF molecules share relatively high homology between each other at the helical regions. They also share acidic residues such as Asp and Glu, involved in the ligand-receptor binding, with mammalian G-CSF [49, 50]. The four carp G-CSF paralogs identified in carp may have arisen from an ancestral G-CSF molecule through a series of duplications, including the teleost-specific $3^{\text {rd }}$ WGD event and the carp-specific $4^{\text {th }}$ WGD event $[16,21,51-53]$. Overall, despite the overall low homology of teleost fish G-CSF sequences with mammalian G-CSF molecules, our in-silico analyses provide clear evidence that all four paralogs identified in carp are indeed orthologs of mammalian G-CSF.

Carp and other teleost fish G-CSF paralogs share only limited conservation of cysteine residues responsible for disulfide bonds with tetrapod G-CSF. Carp G-CSFa1 and G-CSFb1 express two structural differences: (i) an additional helix enriched with acidic residues in G-CSFa1 and (ii) a location of conserved cysteine residues. These structural differences prompted us to further investigate function of the different paralogs. Where $g$-csfa 1 and $g$-csfa2 were highly expressed at basal level especially in spleen, $g$-csfb1 and $g$-csfb2 basal expression levels were very low 
in all tissues examined, indicating that g-csf transcription is differentially regulated between paralogs. Similarly, basal $g$-csfa gene expression was markedly higher than $g$-csfb expression in all immune cells examined. Macrophages are known to be the major cellular source of mammalian G-CSF [2]. Strikingly, G-CSF paralogs were always highest expressed in macrophages of carp.

Basal gene expression levels of $g$-csfa1 in macrophages were higher than those of $g$-csfa2 and gene expression levels of $g$-csfb1 in macrophages were higher than those of $g$-csfb2, which prompted us to further investigate function of G-CSFa1 and $\mathrm{G}-\mathrm{CSFb} 1$ by production of these molecules as recombinant proteins. Recombinant proteins were produced in a bacterial expression system with the limitation that proteins are non-glycosylated and could possibly be contaminated with traces derived from bacteria. However, previous studies on mammalian G-CSF reported that glycosylation is not required for its activity and indeed, the non-glycosylated form is utilized in recombinant therapeutics [54]. Even though the relative insensitivity to LPS has been reported in fish living in the aquatic environment with high pathogenic pressure [55], the recombinant proteins used in our assays were extensively purified up to the absence of LPS traces. Similar to mammalian G-CSF and zebrafish G-CSF $[16,56]$, carp G-CSF induced proliferation of hematopoietic cells in a dose-dependent manner, albeit with apparent different activities for the two paralogs studied: G-CSFa1 induced proliferation of blast-like cells adhesive to culture dishes, whereas G-CSFb1 induced proliferation of cells with neutrophil characteristics. Indeed, treatment with G-CSFb1 showed up-regulation of the transcription factor cebpa involved in neutrophil development [33]. Also, we investigated at least two carp G-CSF receptor genes (data not shown) and found that only G-CSFb1 enhanced gcsfr1 and myeloperoxidase ( Our data indicate that G-CSFb1 and G-CSFR1 are the main players involved with neutrophil development in carp. In zebrafish, both G-CSFa and G-CSFb may bind to the G-CSF receptor, expressed in both neutrophils and macrophages, and promote cell proliferation [16]. In contrast to the latter study, carp G-CSFa1 alone did not stimulate colony formation in our semi-solid culture system, in which an agarose layer prevented natural formation of a stromal and an adherent cell layer. This possibly restricted access to spontaneously secreted growth factors from adherent macrophages [57], which are possibly required for colony formation. Further studies would be required to determine if G-CSFa1 directly induces macrophages to produce autocrine growth factors or that G-CSFa1 synergizes with some factors 
spontaneously secreted from adherent macrophages to synergistically stimulate macrophage development. Meanwhile, carp G-CSFb1 alone did stimulate CFU-G colony formation, whereas the combination of G-CSFa1 and G-CSFb1 stimulated formation of not only CFU-G but also CFU-GM colonies. Our data indicate that carp G-CSFb1 may drive granulopoiesis restricted to neutrophil-lineage development, whereas carp G-CSFa1 may be a cytokine with proliferative effect stimulating CFU-GM or earlier stem/progenitor cells. The functional differences between the G-CSFa1 and G-CSFb1 cytokine preparations make it highly unlikely that the induced cell responses could be due to traces of bacterial contaminations and thus appear cytokine-specific. No matter the indicative differences in biological function between paralogs, carp G-CSFs appears to act as a hematopoietic growth factors.

Mammalian G-CSF is chemo-attractive to neutrophils [58, 59]. In zebrafish, $\mathrm{G}$-CSFb but not G-CSFa could be linked to in vivo trafficking of neutrophils to the site of severe injury [19]. Our results indicate that carp kidney neutrophils are strongly attracted to G-CSFb1 and are moderately attracted to G-CSFa1, possibly under influence of IL-8 (or CXCL8) [40, 60, 61]. Indeed, treatment of carp kidney neutrophil with G-CSF paralogs showed a significant up-regulation of CXCR1 as the IL-8 receptor required for neutrophil recruitment, but not CXCR2 required for neutrophil reverse migration and resolution [45]. Unlike mammalian G-CSF, carp G-CSF paralogs did not mediate transcription of CXCR4, important for retention of neutrophils in the hematopoietic tissue in mammalian models [62]. In conclusion, carp G-CSFb appears to be the most important G-CSF paralog to induce neutrophil migration.

Once neutrophils receive inflammatory cytokine signals, they become 'primed' and capable of promptly and vigorously exerting antimicrobial responses [63]. We could not find a significant change of phagocytic activity in neutrophils against beads and zymosan particles following stimulation of G-CSF paralogs for any period tested (data not shown), indicating that neutrophil phagocytosis is regulated by other signals in fish. Although mammalian G-CSF alone is not able to initiate a respiratory burst in naïve neutrophils, pre-incubation with this cytokine primes the cell for an enhanced superoxide anion production following stimulation with physiological stimuli such as fMLP and PMA $[11,64]$. In fish, following stimulation of phagocytes with inflammatory cytokines, ROS production is activated through at least three sequential steps: (i) activation of protein kinase C (PKC), (ii) phosphorylation of 
p4 $7^{\text {phox }}$ and (iii) the production of ROS catalyzed by the NADPH oxidase complex [48]. In our hands, expression analysis of NADPH oxidase components in neutrophils treated with carp G-CSF paralogs exhibited up-regulation of especially $\mathrm{p} 47^{\text {phox }}$, indicating that the priming effect of carp G-CSF paralogs on neutrophils was regulated through the increase of $\mathrm{p} 47^{\text {phox }}$. Our study provides the first report in teleost fish on the priming effects of G-CSF on neutrophils and analysis of respiratory burst indicated that G-CSFb1 primed neutrophils more effectively than G-CSFa1.

Previous studies in cyprinids showed that circulating blood neutrophils increased in number 6 to $18 \mathrm{~h}$ after i.p. injection with killed E. coli or zymosan and then quickly decreased after $24 \mathrm{~h}$, indicating that an intraperitoneal inflammation in fish induces a temporal mobilization of kidney-derived neutrophils into the circulation [42, 65]. In our study, administration of G-CSF paralogs increased the number of circulating blood neutrophils 6 and $24 \mathrm{~h}$ after i.p. injection, suggesting that also in vivo G-CSFa1 and G-CSFb1 work as chemoattractants and granulopoietic growth factors, in agreement with the in vitro results. However, it remains unclear whether in vivo excess of G-CSF paralogs induce the expression of other inflammatory cytokines and/or chemokines in immune cells. In human clinical medicine, recombinant G-CSF is used as a biophylatic agent to specifically induce granulopoiesis in patients with chemotherapy- and radiation-induced neutropenia to prevent bacterial and fungal infections [66]. Further studies will be required to investigate if fish G-CSF paralogs can act as biophylatic agents against infectious diseases in a similar way. Here, functional analyses were limited to G-CSFa1 and G-CSFb1, and we can only speculate that G-CSFa2 and G-CSFb2 could have similar, different, or combinatorial functions in common carp. Additional biochemical investigations involving all native carp G-CSF paralog proteins will be needed to elucidate the full and complicated picture of immune regulation in this polyploid species.

In summary, we identified four carp G-CSF paralogs, studied their gene expression patterns and characterized the functional differences between $\mathrm{A}$ and $\mathrm{B}$ types of G-CSF on carp hematopoietic cells and neutrophils. We report important differences in their regulation: A type G-CSFs have a relatively high constitutive gene expression and could thus be involved in maintenance of a homeostatic state, whereas B type G-CSFs have a low gene expression and require induction and could 
thus have a responsive, immunological role associated with a state of infection. In general, G-CSFa1 alone stimulates proliferation of granulocyte/macrophage progenitors, while G-CSFb1 promotes proliferation, differentiation and colony formation of granulocyte/macrophage progenitors and granulocyte progenitors in kidney of carp, similar to the G-CSF mammalian counterpart. G-CSFa1 and $\mathrm{G}-\mathrm{CSFb} 1$ act as chemo-attractants to neutrophils modulating the expression of the chemokine receptor CXCR1, suggesting a role for G-CSF paralogs in neutrophil trafficking. Both, G-CSFa1 and G-CSFb1 appear to induce neutrophil 'priming'. The carp G-CSF paralogs reported herein provide us with valuable tools to further study the immune system of teleost fish.

\section{Abbreviations}

WGD, whole genome duplication; LPS, lipopolysaccharide; ConA, concanavaline A; PMA, phorbol 12-myristate 13-acetate; polyI:C, polyinosinic-polycytidylic acid; IPTG, isopropyl $\beta{ }_{\mathrm{D}}{ }^{-}{ }_{\mathrm{L}}$-thiogalactopyranoside; MTT, 3-(4, 5-dimethyl-2-thiazolyl)-2, 5-diphenyltetrazolium bromide; $\mathrm{CFU}-\mathrm{G}$, granulocyte colony-forming unit; $\mathrm{CFU}-$ GM, granulocyte/macrophage colony-forming unit; fMLP, N-formyl-methionylleucyl-phenylalanine; DHR123, dihydrorhodamine 123; FITC, fluorescein isothiocyanate

\section{Acknowledgements}

The authors greatly appreciate Dr. Maria Forlenza and Mr. Jules Petit (Wageningen University) for helpful discussion and advice.

\section{Funding}

This work was supported in part by a Grant-in Aid for Young Scientists (B) (16K18752) from the Japan Society for the Promotion of Science (JSPS) to FK and a grant of International joint research and training of young researchers for zoonosis control in the globalized world (S1491007) from the Ministry of Education, Culture, Sports, Science and Technology (MEXT) Japan. ASW was funded by the European Commission under the $8^{\text {th }}$ (H2020) Framework Program for Research and Technological Development of the European Union (PARAFISHCONTROL Grant No. 634429). 
Chapter 4

\section{Author contributions}

Conceived and designed the experiments: FK and KN. Performed the experiments: FK, KN, AW, and EH. Analyzed the data: FK, KN, AW, JM, and MO. Wrote and edited the paper: FK, KN, AW, GFW and TM. Both FK and KN contributed equally to this work.

\section{Conflict of Interest}

The authors declare that the research was conducted in the absence of any commercial or financial relationships that could be construed as a potential conflict of interest. 


\section{References}

1. Panopoulos AD and Watowich SS (2008) Granulocyte colony-stimulating factor: molecular mechanisms of action during steady state and 'emergency' hematopoiesis. Cytokine 42:277288. doi: 10.1016/j.cyto.2008.03.002.

2. Oster W, Lindemann A, Mertelsmann R and Herrmann F (1989) Production of macrophage-, granulocyte-, granulocyte-macrophage- and multi-colony-stimulating factor by peripheral blood cells. Eur J Immunol 19:543-548. doi: 10.1002/eji.1830190320

3. Koeffer HP, Gasson J, Ranyard J, Souza L, Shepard M and Munker R (1987) Recombinant human TNF alpha stimulates production of granulocyte colony-stimulating factor. Blood 7-:55-59.

4. Zsebo KM, Yuschenkoff VN, Schiffer S, Chang D, McCall E, Dinarello CA, Brown MA, Altrock B and Bagby GC Jr. (1988) Vascular endothelial cells and granulopoiesis: interleukin-1 stimulates release of G-CSF and GM-CSF. Blood 71:99-103.

5. Rennick D, Yang G, Gemmell L and Lee F (1987) Control of hemopoiesis by a bone marrow stromal cell clone: lipopolysaccharide-and interleukin-1-inducible production of colonystimulating factors. Blood 69:682-691.

6. Watari K, Asano S, Shirafuji N, Kodo H, Ozawa K, Takaku F and Kamachi S (1989) Serum granulocyte colony-stimulating factor levels in healthy volunteers and patients with various disorders as estimated by enzyme immunoassay. Blood 73:117-122.

7. Kawakami M, Tsutsumi H, Kumakawa T, Abe H, Hirai M, Kurosawa S, Mori M and Fukushima M (1990) Levels of serum granulocyte colony-stimulating factor in patients with infections. Blood 76:1962-1964.

8. Demetri GD and Griffin JD (1991) Granulocyte colony-stimulating factor and its receptor. Blood 78:2791-2808.

9. Lieschke GJ, Grail D, Hodgson G, Metcalf D, Stanley E, Cheers C, Fowler KJ, Basu S, Zhan YF and Dunn AR (1994) Mice lacking granulocyte colony-stimulating factor have chronic neutropenia, granulocyte and macrophage progenitor cell deficiency, and impaired neutrophil mobilization. Blood 84:1737-1746.

10. Liu F, Wu HY, Wesselschmidt R, Kornaga T and Link DC (1996) Impaired production and increased apoptosis of neutrophils in granulocyte colony-stimulating factor receptor-deficient mice. Immunity 5:491-501. doi: 10.1016/S1074-7613(00)80504-X.

11. Spiekermann K, Roesler J, Emmendoerffer A, Elsner J and Welte K. (1997) Functional features of neutrophils induced by G-CSF and GM-CSF treatment: differential effects and clinical implications. Leukemia (08876924) 11:466-78. doi: 10.1038/sj.leu.2400607.

12. Nicola NA, Metcalf D, Matsumoto M and Johnson GR (1983) Purification of a factor inducing differentiation in murine myelomonocytic leukemia cells. Identification as granulocyte colonystimulating factor. J Biol Chem 258:9017-9023.

13. Santos MD, Yasuike M, Hirono I and Aoki T (2006) The granulocyte colony-stimulating factors (CSF3s) of fish and chicken. Immunogenetics 58:422-432. doi: 10.1007/s00251-006-0106-5.

14. Yaparla A, Wendel ES, and Grayfer L (2016) The unique myelopoiesis strategy of the amphibian Xenopus laevis. Dev Comp Immunol 63:136-43. doi: 10.1016/j.dci.2016.05.014. 
15. Nam BH, An GH, Baeck GW, Kim MC, Kim JW, Park HJ, Lee DC and Park CI (2009) Molecular cloning and expression of cDNAs for two distinct granulocyte colony stimulating factor genes from black rockfish Sebastes schlegelii. Fish Shellfish Immunol 27:360-4. doi: 10.1016/j. fsi.2009.06.005.

16. Stachura DL, Svoboda O, Campbell CA, Espín-Palazón R, Lau RP, Zon LI, Bartunek P and Traver D (2013) The zebrafish granulocyte colony-stimulating factors (Gcsfs): 2 paralogous cytokines and their roles in hematopoietic development and maintenance. Blood 122:3918-3928. doi: 10.1182/blood2012-12-475392.

17. Inoue J, Sato Y, Sinclair R, Tsukamoto K and Nishida M. (2015) Rapid genome reshaping by multiple-gene loss after whole-genome duplication in teleost fish suggested by mathematical modeling. Proc Natl Acad Sci USA 112:14918-14923. doi: 10.1073/pnas.1507669112.

18. Liongue C, Hall CJ, O’Connell BA, Crosier P and Ward AC (2009) Zebrafish granulocyte colonystimulating factor receptor signaling promotes myelopoiesis and myeloid cell migration. Blood 113:2535-46. doi: 10.1182/blood-2008-07-171967.

19. Galdames JA, Zuñiga-Traslaviña C, Reyes AE and Feijóo CG (2014) Gcsf-Chr19 promotes neutrophil migration to damaged tissue through blood vessels in zebrafish. J Immunol 193:3728. doi: 10.4049/jimmunol.1303220.

20. Kolder IC, van der Plas-Duivesteijn SJ, Tan G, Wiegertjes GF, Forlenza M, Guler AT, Travin DY, Nakao M, Moritomo T, Irnazarow I, den Dunnen JT, Anvar SY, Jansen HJ, Dirks RP, Palmblad M, Lenhard B, Henkel CV and Spaink HP (2016) A full-body transcriptome and proteome resource for the European common carp. BMC Genomics 17:701. doi: 10.1186/s12864-016-3038-y.

21. Petit J, David L, Dirks R and Wiegertjes GF (2017) Genomic and transcriptomic approaches to study immunology in cyprinids: What is next? Dev Comp Immunol 75:48-62. doi: 10.1016/j. dci.2017.02.022.

22. Katzenback BA and Belosevic M (2009) Isolation and functional characterization of neutrophillike cells, from goldfish (Carassius auratus L.) kidney. Dev Comp Immunol 33:601-11. doi: 10.1016/j.dci.2008.10.011.

23. Stolte EH, Savelkoul HF, Wiegertjes G, Flik G and Verburg-van Kemenade BML (2008) Differential expression of two interferon-gamma genes in common carp (Cyprinus carpio L.). Dev Comp Immunol 32:1467-81. doi: 10.1016/j.dci.2008.06.012.

24. Joerink M, Ribeiro CM, Stet RJ, Hermsen T, Savelkoul HF and Wiegertjes GF (2006) Head KidneyDerived Macrophages of Common Carp (Cyprinus carpio L.) Show Plasticity and Functional Polarization upon Differential Stimulation. The Journal of Immunology 177:61-69. doi: 10.4049/ jimmunol.177.1.61.

25. Forlenza M, Scharsack JP, Kachamakova NM, Taverne-Thiele AJ, Rombout JH and Wiegertjes GF (2008) Differential contribution of neutrophilic granulocytes and macrophages to nitrosative stress in a host-parasite animal model. Mol Immunol 45:3178-89. doi: 10.1016/j. molimm.2008.02.025.

26. Secombes C, Van Groningen J, and Egberts E (1983) Separation of lymphocyte subpopulations in carp Cyprinus carpio L. by monoclonal antibodies: immunohistochemical studies. Immunology 48:165. 
27. Rombout JHWM, Koumans-van Diepen JCE, Emmer PM, Taverne-Thiele JJ and Taverne N (1996) Characterization of carp thrombocytes with specific monoclonal antibodies. J Fish Biol 49:521-531. doi: 0.1111/j.1095-8649.1996.tb00047.x

28. Katakura F, Yabu T, Yamaguchi T, Miyamae J, Shirinashihama Y, Nakanishi T and Moritomo $\mathrm{T}$ (2015) Exploring erythropoiesis of common carp (Cyprinus carpio) using an in vitro colony assay in the presence of recombinant carp kit ligand A and erythropoietin. Dev Comp Immunol 53:13-22. doi: 10.1016/j.dci.2015.06.006.

29. Katakura F, Sugie Y, Hayashi K, Nishiya K, Miyamae J, Okano M, Nakanishi T and Moritomo $\mathrm{T}$ (2018) Thrombopoietin (TPO) induces thrombocytic colony formation of kidney cells synergistically with kit ligand A and a non-secretory TPO variant exists in common carp. Dev Comp Immunol 84:327-336. doi: 10.1016/j.dci.2018.03.005

30. Katakura F, Yamaguchi T, Yoshida M, Moritomo T and Nakanishi T. (2010) Demonstration of $\mathrm{T}$ cell and macrophage progenitors in carp (Cyprinus carpio) kidney hematopoietic tissues. Development of clonal assay system for carp hematopoietic cells. Dev Comp Immunol 34:685689. doi: doi: 10.1016/j.dci.2010.01.015

31. Tsai F-Y and Orkin SH (1997) Transcription factor GATA-2 is required for proliferation/survival of early hematopoietic cells and mast cell formation, but not for erythroid and myeloid terminal differentiation. Blood 89:3636-3643.

32. Dahl R, Walsh JC, Lancki D, Laslo P, Iyer SR, Singh H and Simon MC (2003) Regulation of macrophage and neutrophil cell fates by the PU.1:C/EBPalpha ratio and granulocyte colonystimulating factor. Nat Immunol 4:1029-36. doi: 10.1038/ni973.

33. Kurotaki D, Yamamoto M, Nishiyama A, Uno K, Ban T, Ichino M, Sasaki H, Matsunaga S, Yoshinari M, Ryo A, Nakazawa M, Ozato K and Tamura T (2014) IRF8 inhibits C/EBPalpha activity to restrain mononuclear phagocyte progenitors from differentiating into neutrophils. Nat Commun 5:4978. doi: 10.1038/ncomms5978.

34. Ma O, Hong S, Guo H, Ghiaur G and Friedman AD. (2014) Granulopoiesis requires increased C/ EBP $\alpha$ compared to monopoiesis, correlated with elevated Cebpa in immature G-CSF receptor versus M-CSF receptor expressing cells. PLoS One 9:e95784. doi: 10.1371/journal.pone.0095784

35. Katzenback BA, Katakura F, and Belosevic M (2016) Goldfish (Carassius auratus L.) as a model system to study the growth factors, receptors and transcription factors that govern myelopoiesis in fish. Dev Comp Immunol 58:68-85. doi: 10.1016/j.dci.2015.10.024.

36. Rhodes J, Hagen A, Hsu K, Deng M, Liu TX, Look AT and Kanki JP (2005) Interplay of pu.1 and gata1 determines myelo-erythroid progenitor cell fate in zebrafish. Dev Cell 8:97-108. doi: 10.1016/j.devcel.2004.11.014.

37. Nutt SL, Heavey B, Rolink AG and Busslinger M (1999) Commitment to the B-lymphoid lineage depends on the transcription factor Pax5. Nature 204:14-20. doi: 10.1038/35005514.

38. Hosoya T, Maillard I, and Engel JD (2010) From the cradle to the grave: activities of GATA3 throughout T-cell development and differentiation. Immunol Rev 238:110-125. doi: 10.1111/j.1600-065X.2010.00954.x. 
39. Fujiki K, Shin DH, Nakao M and Yano T (1999) Molecular cloning of carp (Cyprinus carpio) CC chemokine, CXC chemokine receptors, allograft inflammatory factor-1, and natural killer cell enhancing factor by use of suppression subtractive hybridization. Immunogenetics 490:909-914. doi: 10.1007/s002510050573.

40. van der Aa LM, Chadzinska M, Tijhaar E, Boudinot P and Verburg-van Kemenade BML (2010) CXCL8 chemokines in teleost fish: two lineages with distinct expression profiles during early phases of inflammation. PLoS One 5:e12384. doi: 10.1371/journal.pone.0012384.

41. Mayumi M, Takeda Y, Hoshiko M, Serada K, Murata M, Moritomo T, Takizawa F, Kobayashi I, Araki K, Nakanishi T and Sumimoto H. (2008) Characterization of teleost phagocyte NADPH oxidase: molecular cloning and expression analysis of carp (Cyprinus carpio) phagocyte NADPH oxidase. Mol Immunol 45:1720-31. doi: 10.1016/j.molimm.2007.09.028.

42. Havixbeck JJ, Rieger AM, Wong ME, Hodgkinson JW and Barreda DR (2016) Neutrophil contributions to the induction and regulation of the acute inflammatory response in teleost fish. J Leukoc Biol 99:241-52. doi: 10.1189/jlb.3HI0215-064R.

43. Nakayasu C, Omori M, Hasegawa S, Kurata O and Okamoto N (1998) Production of a monoclonal antibody for carp (Cyprinus carpio L.) phagocytic cells and separation of the cells. Fish Shellfish Immunol 8:91-100. doi: 10.1006/fsim.1997.0125.

44. Blaser BW, Moore JL, Hagedorn EJ, Li B, Riquelme R, Lichtig A, Yang S, Zhou Y, Tamplin OJ, Binder V and Zon LI (2017) CXCR1 remodels the vascular niche to promote hematopoietic stem and progenitor cell engraftment. J Exp Med 214:1011-1027. doi: 10.1084/jem.20161616.

45. Powell D, Tauzin S, Hind LE, Deng Q, Beebe DJ and Huttenlocher A (2017) Chemokine Signaling and the Regulation of Bidirectional Leukocyte Migration in Interstitial Tissues. Cell Rep 19:1572-1585. doi: 10.1016/j.celrep.2017.04.078.

46. Babior BM, Kipnes RS, and Curnutte JT (1973) Biological defense mechanisms. The production by leukocytes of superoxide, a potential bactericidal agent. J Clin Invest 52:741. doi: 10.1172/ JCI107236.

47. Hampton MB, Kettle AJ, and Winterbourn CC (1998) Inside the Neutrophil Phagosome: Oxidants, Myeloperoxidase, and Bacterial Killing. Blood 92:3007-3017. doi:

48. Olavarría VH, Gallardo L, Figueroa JE and Mulero V. (2010) Lipopolysaccharide primes the respiratory burst of Atlantic salmon SHK-1 cells through protein kinase C-mediated phosphorylation of p47phox. Dev Comp Immunol 34:1242-53. doi: 10.1016/j.dci.2010.07.002.

49. Aritomi M, Kunishima N, Okamoto T, Kuroki R, Ota Y and Morikawa K (1999) Atomic structure of the GCSF-receptor complex showing a new cytokine-receptor recognition scheme. Nature 401:713-717. doi: 10.1038/44394.

50. Layton JE, Hall NE, Connell F, Venhorst J and Treutlein HR (2001) Identification of ligandbinding site III on the immunoglobulin-like domain of the granulocyte colony-stimulating factor receptor. J Biol Chem 276:36779-87. doi: 10.1074/jbc.M104787200.

51. Hultman KA, Bahary N, Zon LI and Johnson SL (2007) Gene Duplication of the zebrafish kit ligand and partitioning of melanocyte development functions to kit ligand a. PLoS Genet 3:e17. doi: 10.1371/journal.pgen.0030017. 
52. Wang T, Hanington PC, Belosevic M and Secombes CJ (2008) Two Macrophage ColonyStimulating Factor Genes Exist in Fish That Differ in Gene Organization and Are Differentially Expressed. J Immunol 181:3310-3322. doi: 10.4049/jimmunol.181.5.3310.

53. Zou J and Secombes CJ (2016) The Function of Fish Cytokines. Biology 5:23. doi: 10.3390/ biology5020023.

54. Bönig H, Silbermann S, Weller S, Kirschke R, Körholz D, Janssen G, Göbel U and Nürnberger W (2001) Glycosylated vs non-glycosylated granulocyte colony-stimulating factor (G-CSF)results of a prospective randomised monocentre study. Bone marrow transplant 28:259. doi: 10.1038/sj.bmt.1703136.

55. Novoa B, Bowman TV, Zon L and Figueras A (2009) LPS response and tolerance in the zebrafish (Danio rerio). Fish Shellish Immunol 26:326-331. doi: 10.1016/j.fsi.2008.12.004.

56. Souza LM, Boone TC, Gabrilove J, Lai PH, Zsebo KM, Murdock DC, Chazin VR, Bruszewski J, Lu H, Chen KK, Barendt J, Platzer E, Moore MAS, Mertelsmann R and Welte K (1986) Recombinant human granulocyte colony-stimulating factor: effects on normal and leukemic myeloid cells. Science 232:6-5. doi: 10.1126/science.24 20009.

57. Neumann NF, Barreda D, and Belosevic M (1998) Production of a macrophage growth factor (s) by a goldfish macrophage cell line and macrophages derived from goldfish kidney leukocytes. Dev Comp Immunol 22:417-432. doi: 10.1016/S0145-305X(98)00023-8.

58. Wang JM, Chen ZG, Colella S, Bonilla MA, Welte K, Bordignon C and Mantovani A (1988) Chemotactic activity of recombinant human granulocyte colony-stimulating factor. Blood 72:1456-1460.

59. Semerad CL, Liu F, Gregory AD, Stumpf K and Link DC (2002) G-CSF is an essential regulator of neutrophil trafficking from the bone marrow to the blood. Immunity 17:413-423. doi: 10.1016/ S1074-7613(02)00424-7

60. de Oliveira S, Reyes-Aldasoro CC, Candel S, Renshaw SA, Mulero V and Calado A (2013) Cxcl8 (IL-8) mediates neutrophil recruitment and behavior in the zebrafish inflammatory response. $J$ Immunol 190:4349-59. doi: 10.4049/jimmunol.1203266.

61. de Oliveira S, Lopez-Muñoz A, Martínez-Navarro FJ, Galindo-Villegas J, Mulero V and, Calado $\hat{A}$ (2015) Cxcl8-11 and Cxcl8-12 are required in the zebrafish defense against Salmonella Typhimurium. Dev Comp Immunol 49:44-8. doi: 10.1016/j.dci.2014.11.004.

62. Summers C, Rankin SM, Condliffe AM, Singh N, Peters AM and Chilvers ER (2010) Neutrophil kinetics in health and disease. Trends Immunol 31:318-24. doi: 10.1016/j.it.2010.05.006.

63. Hallett MB and Lloyds D (1995) Neutrophil priming: the cellular signals that say 'amber' but not 'green'. Immunology today 16:264-268.

64. Kitagawa S, Yuo A, Souza LM, Saito M, Miura Y and Takaku F (1987) Recombinant human granulocyte colony-stimulating factor enhances superoxide release in human granulocytes stimulated by the chemotactic peptide. Biochem Biophys res commun 144:1143-1146. doi: 10.1016/0006-291X(87)91430-6.

65. Kodama H, Tijiwa K, Moritomo $\mathrm{T}$ and Nakanishi $\mathrm{T}$ (2002) Granulocyte responses to experimental injection of live and formalin-killed bacteria in carp (Cyprinus carpio). Vet Immunol Immunopathol 90:101-105. doi: 10.1016/S0165-2427(02)00230-1. 


\section{Chapter 4}

66. Aapro MS, Bohlius J, Cameron DA, Dal Lago L, Donnelly JP, Kearney N, Lyman GH, Pettengell R, Tjan-Heijnen VC, Walewski J, Weber DC and Zielinski C; European Organisation for Research and Treatment of Cancer (2011) 2010 update of EORTC guidelines for the use of granulocytecolony stimulating factor to reduce the incidence of chemotherapy-induced febrile neutropenia in adult patients with lymphoproliferative disorders and solid tumours. Eur J Cancer 47:8-32. doi: 10.1016/j.ejca.2010.10.013. 


\section{Supplementary Material: Paralogs of common carp granulocyte colony-stimulating factor (G-CSF) have different functions with regard to development, trafficking and activation of neutrophils}

Supplementary table S4.1. Amino acid sequence identities (upper grids) and similarities (lower grids) of human, mouse, chicken, xenopus, and fish G-CSF orthologues. Amino acid identities and similarities (in percentages) were calculated using the Sequence Manipulation Suite v2: Ident and Sim (http://www.bioinformatics.org/sms2/ident_sim.html) following the multiple alignment with the Clustal Omega (https://www.ebi.ac.uk/Tools/msa/clustalo/) using default parameters. Accession numbers of protein sequences listed are same to Figure 4.1B.

\begin{tabular}{|lcccccccccc|}
\hline & Human & Mouse & Chicken & $\begin{array}{c}\text { African- } \\
\text { clawed } \\
\text { frog }\end{array}$ & $\begin{array}{c}\text { Gebrafish } \\
\text { G-CSFa }\end{array}$ & Carp & Carp & Zebrafish & Carp & Carp \\
Human & & $\mathbf{6 9 . 9 5}$ & $\mathbf{3 5 . 2 1}$ & $\mathbf{2 2 . 8 6}$ & $\mathbf{1 6 . 0 7}$ & $\mathbf{1 4 . 4 0}$ & $\mathbf{1 3 . 8 8}$ & $\mathbf{1 8 . 6 4}$ & $\mathbf{2 0 . 8 1}$ & $\mathbf{2 1 . 8 2}$ \\
\hline Mouse & 76.06 & & $\mathbf{3 3 . 0 2}$ & $\mathbf{2 4 . 0 4}$ & $\mathbf{1 3 . 7 8}$ & $\mathbf{1 4 . 8 1}$ & $\mathbf{1 3 . 3 6}$ & $\mathbf{1 5 . 1 1}$ & $\mathbf{1 6 . 3 7}$ & $\mathbf{1 7 . 7 8}$ \\
\hline Chicken & 49.77 & 49.06 & & $\mathbf{2 5 . 3 7}$ & $\mathbf{1 4 . 2 9}$ & $\mathbf{1 5 . 2 5}$ & $\mathbf{1 4 . 7 1}$ & $\mathbf{1 5 . 2 8}$ & $\mathbf{1 8 . 8 9}$ & $\mathbf{1 9 . 3 5}$ \\
\hline $\begin{array}{l}\text { African- } \\
\text { clawed frog }\end{array}$ & 31.90 & 37.50 & 39.02 & & $\mathbf{1 5 . 8 1}$ & $\mathbf{1 3 . 2 5}$ & $\mathbf{1 3 . 5 6}$ & $\mathbf{1 3 . 6 2}$ & $\mathbf{1 3 . 5 5}$ & $\mathbf{1 4 . 5 5}$ \\
\hline $\begin{array}{l}\text { Zebrafish } \\
\text { G-CSFa }\end{array}$ & 29.91 & 29.78 & 30.41 & 29.77 & & $\mathbf{5 6 . 7 0}$ & $\mathbf{5 1 . 3 2}$ & $\mathbf{1 8 . 8 7}$ & $\mathbf{2 8 . 2 3}$ & $\mathbf{2 7 . 7 5}$ \\
\hline Carp G-CSFa1 & 29.63 & 30.86 & 31.78 & 28.63 & 68.30 & & $\mathbf{6 9 . 7 4}$ & $\mathbf{1 8 . 6 1}$ & $\mathbf{2 8 . 0 7}$ & $\mathbf{2 8 . 9 5}$ \\
\hline Carp G-CSFa2 & 27.35 & 28.34 & 30.67 & 26.69 & 60.96 & 75.44 & & $\mathbf{1 9 . 9 1}$ & $\mathbf{2 8 . 3 8}$ & $\mathbf{2 9 . 2 6}$ \\
\hline $\begin{array}{l}\text { Zebrafish } \\
\text { G-CSFb }\end{array}$ & 30.00 & 26.22 & 30.09 & 23.47 & 34.43 & 34.20 & 35.50 & & $\mathbf{3 4 . 3 4}$ & $\mathbf{3 6 . 3 6}$ \\
\hline Carp G-CSFb1 & 37.10 & 34.51 & 36.87 & 27.10 & 44.98 & 44.30 & 44.54 & 53.54 & & $\mathbf{7 2 . 8 2}$ \\
\hline Carp G-CSFb2 & 36.82 & 34.22 & 34.10 & 30.05 & 45.93 & 44.74 & 45.85 & 52.53 & 82.05 & \\
\hline
\end{tabular}

Supplementary table S4.2. Oligonucleotide primers used for cDNA cloning from carp and construction of expression plasmid vectors.

\begin{tabular}{|lll|}
\hline Primer name & Primer nucleotide sequences (5'-3') & Application \\
\hline g-csfa1 F1 & CACACTTGTGGGAATTGTGG & Partial sequencing \\
\hline g-csfa1 R1 & TACTGCGGAGGATGTCACAG & Partial sequencing \\
\hline g-csfa1_3' F1 & GCATCTGCCCCAATCTCTGACAAAC & 3' RACE \\
\hline g-csfa1_3' F2 & GGCTCAGACAGACCAAGTAAAAGACC & 3' RACE (nested PCR) \\
\hline g-csfa1_5' R1 & ACATGCAGGTTGACAAGCAA & 5' RACE \\
\hline g-csfa1_5' R2 & TCTGATGCCTGCTTTGATGGGTCA & 5' RACE (nested PCR) \\
\hline g-csfa1_full F & ATGGGGACTGCTGCAATCTG & CDS cloning \\
\hline g-csfa1_full R & CATGTATGCTAGGGTACAATGCTG & CDS cloning \\
\hline g-csfa2_full F & CATGTTCTTTTTGATTACTGCTGTG & CDS cloning \\
\hline g-csfa2_full R & AAGGGAGTTCCTAATGTGTTAAATTAA & CDS cloning \\
\hline g-csfb1_3' F1 & CGCGCCACGAGTTTAGCCAAGAAG & 3' RACE \\
\hline
\end{tabular}




\begin{tabular}{|lll|}
\hline Primer name & Primer nucleotide sequences (5'-3') & Application \\
\hline g-csfb1_3' F2 & TCACACAGGACGTCTTCCGCAGTC & 3' RACE (nested PCR) \\
\hline g-csfb1_5' R1 & AGACTGCGGAAGACGTCCTGTGTGAA & 5' RACE \\
\hline g-csfb1_5'R2 & CGGCCACAATACGGCTTAAACTC & 5' RACE (nested PCR) \\
\hline g-csfb1_full F & CGCAATAACGAGACAGCTCA & CDS cloning \\
\hline g-csfb1_full R & CACAGGGTACAACATCTGTCAA & CDS cloning \\
\hline g-csfb2_full F & AGCCTGCTAGAAATCCCTTGA & CDS cloning \\
\hline g-csfb2_full R & AAATATAGCAGGGACTGGTTGG & CDS cloning \\
\hline $\begin{array}{l}\text { G-CSFa1__ } \\
\text { ProExp_Ndel_F }\end{array}$ & CATATGGCCCCAATCTCTGA & $\begin{array}{l}\text { Construction of an expression plasmid } \\
\text { vector }\end{array}$ \\
\hline $\begin{array}{l}\text { G-CSFa1_- } \\
\text { ProExp_ } \\
\text { BamHI_R }\end{array}$ & GGATCCTCATAGACCTGCTTTA & $\begin{array}{l}\text { Construction of an expression plasmid } \\
\text { vector }\end{array}$ \\
\hline $\begin{array}{l}\text { G-CSFb1_ } \\
\text { ProExp_Ndel_F }\end{array}$ & CATATGGCGCCGCTCCAG & $\begin{array}{l}\text { Construction of an expression plasmid } \\
\text { vector }\end{array}$ \\
\hline $\begin{array}{l}\text { G-CSFb1_ } \\
\text { ProExp_ } \\
\text { BamHI_R }\end{array}$ & GGATCCCTAGTTTGATGCATCA & $\begin{array}{l}\text { Construction of an expression plasmid } \\
\text { vector }\end{array}$ \\
\hline
\end{tabular}

Supplementary table S4.3 Oligonucleotide primers used for gene expression analysis with quantitative RT-PCR.

\begin{tabular}{|c|c|c|c|}
\hline $\begin{array}{l}\text { Target } \\
\text { (Accession No.) }\end{array}$ & Primer nucleotide sequences (5'-3') & $\begin{array}{l}\text { Product } \\
\text { length }\end{array}$ & Marker for \\
\hline Carp g-csfa1 & F АСССТСTGCCCCAGTTCTTC & \multirow{2}{*}{134 bp } & \multirow[t]{2}{*}{ G-CSFa1 } \\
\hline (MG882495) & R TCTGAGCCAGTGTGGTTGC & & \\
\hline Carp g-csfa2 & F TGGGCGACAACACGATTAGA & \multirow{2}{*}{136 bp } & \multirow[t]{2}{*}{ G-CSFa2 } \\
\hline (MG882496) & R TGAAGTTGCAGTCCCTTCACC & & \\
\hline Carp g-csfb1 & F TGAAGTTTTGCCTCATTCTTGC & \multirow{2}{*}{135 bp } & \multirow[t]{2}{*}{ G-CSFb1 } \\
\hline (MG882497) & R CAACGTCGCTCAGGATCTTCT & & \\
\hline Carp g-csfb2 & F CCACAGAATCCCAGAAAACCA & \multirow{2}{*}{135 bp } & \multirow[t]{2}{*}{ G-CSFb2 } \\
\hline (MG882498) & R GGCGTAGACTGCGGAAGACA & & \\
\hline Carp il-1 $\beta$ & F AAGGAGGCCAGTGGCTCTGT & \multirow{2}{*}{72 bp } & \multirow[t]{2}{*}{ Interleukin-1 beta } \\
\hline (AJ245635) & R CCTGAAGAAGAGGAGGAGGCTGTCA & & \\
\hline Carp cebpa & F AAGACACCGCTGGAGACCTG & \multirow{2}{*}{128 bp } & \multirow{2}{*}{$\begin{array}{l}\text { Transcription factor } \\
\text { (TF) involved in } \\
\text { myelopoiesis }\end{array}$} \\
\hline$(\mathrm{MH} 262559)$ & R TTGCTTGGAGTTGTTGTGGAA & & \\
\hline Carp pu.1 & F ACCGGGCATCACCTCACTCT & \multirow{2}{*}{125 bp } & \multirow{2}{*}{$\begin{array}{l}\text { TF involved in } \\
\text { myelopoiesis }\end{array}$} \\
\hline (XM_019107899) & R CTGCTGGGGTCATCGTCATC & & \\
\hline
\end{tabular}




\begin{tabular}{|c|c|c|c|}
\hline $\begin{array}{l}\text { Target } \\
\text { (Accession No.) }\end{array}$ & Primer nucleotide sequences (5'-3') & $\begin{array}{l}\text { Product } \\
\text { length }\end{array}$ & Marker for \\
\hline $\begin{array}{l}\text { Carp irf8 } \\
\text { (XM_019088951) }\end{array}$ & $\begin{array}{l}\text { F ACTATGGAGGTCGGCTGGTG } \\
\text { R GATGTTCTGGAGGCTGTCTGG }\end{array}$ & 122 bp & $\begin{array}{l}\text { TF involved in } \\
\text { myelopoiesis }\end{array}$ \\
\hline $\begin{array}{l}\text { Carp gata2 } \\
\text { (AB429308) }\end{array}$ & $\begin{array}{l}\text { F CCСАТСССААССТАСССААС } \\
\text { R TCАСАСАТTСАСGССССТСТ }\end{array}$ & $157 \mathrm{bp}$ & $\begin{array}{l}\text { TF involved in early } \\
\text { hematopoiesis }\end{array}$ \\
\hline $\begin{array}{l}\text { Carp gata1 } \\
\text { (AB429307) }\end{array}$ & $\begin{array}{l}\text { F TGAGCCCTTCATCATTCTCC } \\
\text { R TCCGCAAGCATTACAGAGGT }\end{array}$ & $146 \mathrm{bp}$ & $\begin{array}{l}\text { TF involved in } \\
\text { erythropoiesis }\end{array}$ \\
\hline $\begin{array}{l}\text { Carp gata3 } \\
\text { (AB429311) }\end{array}$ & $\begin{array}{l}\text { F TATCGACGGACAGAGCAACC } \\
\text { R CCATCAAGCCAAGGAAGAGA }\end{array}$ & 132 bp & $\begin{array}{l}\text { TF involved in T } \\
\text { lymphopoiesis }\end{array}$ \\
\hline $\begin{array}{l}\text { Carp pax5 } \\
\text { (AB429310) }\end{array}$ & $\begin{array}{l}\text { F CAGCGTCAGCTCAATCAACA } \\
\text { R TACTGCGGATACCTGGGTCA }\end{array}$ & $121 \mathrm{bp}$ & $\begin{array}{l}\text { TF involved in B } \\
\text { lymphopoiesis }\end{array}$ \\
\hline $\begin{array}{l}\text { Carp csf1r } \\
\text { (AB526448) }\end{array}$ & $\begin{array}{l}\text { F CAGGAAACCGGCCACTACA } \\
\text { R CCCATCTCACCATCGCTACA }\end{array}$ & $106 \mathrm{bp}$ & $\begin{array}{l}\text { Colony-stimulating } \\
\text { factor } 1 \text { receptor } \\
\text { (monocyte/ } \\
\text { macrophage) }\end{array}$ \\
\hline $\begin{array}{l}\text { Carp gcsfr1 } \\
(\mathrm{MH} 262557)\end{array}$ & $\begin{array}{l}\text { F TGTTCATACGATGGGTGGAAG } \\
\text { R AACACAGGCAAACACAACGA }\end{array}$ & $145 \mathrm{bp}$ & $\begin{array}{l}\text { G-CSF receptor } 1 \\
\text { (neutrophil) }\end{array}$ \\
\hline $\begin{array}{l}\text { Carp gcsfr2 } \\
(\mathrm{MH} 262558)\end{array}$ & $\begin{array}{l}\text { F GCTGGGCTCTGTCTCCTGTT } \\
\text { R CACTGAGGGATGCTGGTGTT }\end{array}$ & $92 \mathrm{bp}$ & G-CSF receptor 2 \\
\hline $\begin{array}{l}\text { Carp mpx } \\
\text { (AB429306) }\end{array}$ & $\begin{array}{l}\text { F GTGGTCGTGTCGGTCCTCTT } \\
\text { R GATGCCTTTTGTTTGGTGGTG }\end{array}$ & 118 bp & $\begin{array}{l}\text { Myeloperoxidase } \\
\text { (neutrophil) }\end{array}$ \\
\hline $\begin{array}{l}\text { Carp cxcr1 } \\
\text { (AB010468) }\end{array}$ & $\begin{array}{l}\text { F AGACGAATCACGCCGACATA } \\
\text { R GACCACCAGAGGGAAGAGGA }\end{array}$ & $80 \mathrm{bp}$ & $\begin{array}{l}\text { Interleukin-8 receptor } \\
\text { alpha subunit }\end{array}$ \\
\hline $\begin{array}{l}\text { Carp cxcr2 } \\
\text { (AB010713) }\end{array}$ & $\begin{array}{l}\text { F GCCATCGTGAAAGCAACC } \\
\text { R AGGCACATACCCACACCAAC }\end{array}$ & $82 \mathrm{bp}$ & $\begin{array}{l}\text { Interleukin-8 receptor } \\
\text { beta subunit }\end{array}$ \\
\hline $\begin{array}{l}\text { Carp cxcr4 } \\
\text { (AB012310) }\end{array}$ & $\begin{array}{l}\text { F TGACACGGGCATGAATACGA } \\
\text { R ATGTGCTGAAAGCGGAACAC }\end{array}$ & 87 bp & $\begin{array}{l}\text { Receptor for } \\
\text { CXCL12 }\end{array}$ \\
\hline $\begin{array}{l}\text { Carp gp91phox } \\
\text { (AB290328) }\end{array}$ & $\begin{array}{l}\text { F TCATCAAGTGCCCATCCATC } \\
\text { R CCACGGTTTTGTCACCTCCA }\end{array}$ & $150 \mathrm{bp}$ & $\begin{array}{l}\text { Transmembrane } \\
\text { subunit of NADPH } \\
\text { oxidase }\end{array}$ \\
\hline $\begin{array}{l}\text { Carp p22 } 2^{\text {phox }} \\
\text { (AB290329) }\end{array}$ & $\begin{array}{l}\text { F TATGCGACTGGTGGGATTGT } \\
\text { R CTTTGCCCCGTTTGCTTC }\end{array}$ & $124 \mathrm{bp}$ & $\begin{array}{l}\text { Transmembrane } \\
\text { subunit of NADPH } \\
\text { oxidase }\end{array}$ \\
\hline $\begin{array}{l}\text { Carp p47 phox } \\
\text { (AB290330) }\end{array}$ & $\begin{array}{l}\text { F GTGGGTGGTCAGGAAAGGAG } \\
\text { R GGGCGTTGCGTATGGTAGA }\end{array}$ & $146 \mathrm{bp}$ & $\begin{array}{l}\text { Cytosolic subunit of } \\
\text { NADPH oxidase }\end{array}$ \\
\hline $\begin{array}{l}\text { Carp p67 } 7^{\text {phox }} \\
\text { (AB290331) }\end{array}$ & $\begin{array}{l}\text { F AGGCTCAGTTGGGAAAATGG } \\
\text { R TATGGCTGAACTTGGCGTCT }\end{array}$ & $84 \mathrm{bp}$ & $\begin{array}{l}\text { Cytosolic subunit of } \\
\text { NADPH oxidase }\end{array}$ \\
\hline
\end{tabular}




\begin{tabular}{|c|c|c|c|}
\hline $\begin{array}{l}\text { Target } \\
\text { (Accession No.) }\end{array}$ & Primer nucleotide sequences ( $\left.5^{\prime}-3^{\prime}\right)$ & $\begin{array}{l}\text { Product } \\
\text { length }\end{array}$ & Marker for \\
\hline $\begin{array}{l}\text { Carp p40 phox } \\
\text { (AB290332) }\end{array}$ & $\begin{array}{l}\text { F CCCCCACACGCAAAGTAAA } \\
\text { R CTGCCGCTGAAGTCAAACAC }\end{array}$ & $88 \mathrm{bp}$ & $\begin{array}{l}\text { Cytosolic subunit of } \\
\text { NADPH oxidase }\end{array}$ \\
\hline $\begin{array}{l}\text { Carp } \beta \text {-actin } \\
\text { (M24113) }\end{array}$ & $\begin{array}{l}\text { F CAAGGCCAACAGGGAAAAGA } \\
\text { R AGGCATACAGGGACAGCACA }\end{array}$ & $98 \mathrm{bp}$ & Internal control \\
\hline $\begin{array}{l}\text { Carp 40s } \\
\text { (AB012087) }\end{array}$ & $\begin{array}{l}\text { F CCGTGGGTGACATCGTTACA } \\
\text { R TCAGGACATTGAACCTCACTGTCT }\end{array}$ & $69 \mathrm{bp}$ & Internal control \\
\hline
\end{tabular}

Supplementary table S4.4. Oligonucleotide primers used for gene expression analysis with semiquantitative RT-PCR.

\begin{tabular}{|c|c|c|c|}
\hline $\begin{array}{l}\text { Target } \\
\text { (Accession No.) }\end{array}$ & Primer nucleotide sequences (5'-3') & $\begin{array}{l}\text { Product } \\
\text { length }\end{array}$ & Marker for \\
\hline $\begin{array}{l}\text { Carp gcsfr(1/2) } \\
(\mathrm{MH} 262557 / \\
\mathrm{MH} 262558)\end{array}$ & $\begin{array}{l}\text { F GTTGTGTTTGCCTGTGTTGG } \\
\text { R CTGGTGGAGGGGATGAATG }\end{array}$ & $379 \mathrm{bp}$ & Neutrophil \\
\hline $\begin{array}{l}\text { Carp cebpa } \\
(\mathrm{MH} 262559)\end{array}$ & $\begin{array}{l}\text { F GCTGGAGACCTGAGCGAGAT } \\
\text { R GCGTGGTGTTGAGAGTGGTG }\end{array}$ & $353 \mathrm{bp}$ & Neutrophil \\
\hline $\begin{array}{l}\text { Carp } m p x / m p o \\
\text { (AB429306) }\end{array}$ & $\begin{array}{l}\text { F ACCACAGTATACCAGGCTATAATGC } \\
\text { R GGTTCTCAAACCATAACCTGTCC }\end{array}$ & $270 \mathrm{bp}$ & Neutrophil \\
\hline $\begin{array}{l}\text { Carp csf1r/mcsfr } \\
\text { (AB526448) }\end{array}$ & $\begin{array}{l}\text { F AACTAAAGCTCGGAAAGACTCTGG } \\
\text { R CGCAGGAAGTTCAGAAGATCAC }\end{array}$ & $286 \mathrm{bp}$ & $\begin{array}{l}\text { Monocyte/ } \\
\text { macrophage }\end{array}$ \\
\hline $\begin{array}{l}\text { Carp irf8 } \\
\text { (XM_019088951) }\end{array}$ & $\begin{array}{l}\text { F GTGCCAGAGGAGGAACAGAAG } \\
\text { R GATGTTCTGGAGGCTGTCTGG }\end{array}$ & $414 \mathrm{bp}$ & $\begin{array}{l}\text { Monocyte/ } \\
\text { macrophage }\end{array}$ \\
\hline $\begin{array}{l}\text { Carp lck } \\
\text { (AB429309) }\end{array}$ & $\begin{array}{l}\text { F CGTCGGGTGGCTATCAAGAG } \\
\text { R TGAGCTCATCGGACACCAAA }\end{array}$ & $328 \mathrm{bp}$ & T cell \\
\hline $\begin{array}{l}\text { Carp IgM heavy } \\
\text { chain (ighm) } \\
\text { (AB004105) }\end{array}$ & $\begin{array}{l}\text { F TTCTTCСАССАСССССАС } \\
\text { R GCTGCAATCTTGAATAGGAACTG }\end{array}$ & $353 \mathrm{bp}$ & B cell \\
\hline $\begin{array}{l}\text { Carp gata1 } \\
\text { (AB429307) } \\
\end{array}$ & $\begin{array}{l}\text { F TTCCAGCTCTGAGACTGACTTACTGC } \\
\text { R CCCGTATGGACCCAGCATGT }\end{array}$ & $442 \mathrm{bp}$ & Erythrocyte \\
\hline $\begin{array}{l}\text { Carp } \beta \text {-actin } \\
\text { (M24113) }\end{array}$ & $\begin{array}{l}\text { F GTACGTTGCCATCCAGGCTGTG } \\
\text { R ACGTCACACTTCATGATGGAGTTGAAG }\end{array}$ & $465 \mathrm{bp}$ & Internal control \\
\hline
\end{tabular}


ACTGCTGCAATCTGGTGACTTTGCGCATTTTCTTTTTGATCACTGTTGCTATAATGAACTTCCAGggt. . (intron1; 341

$$
\begin{array}{llllll}
M & \mathrm{~N} & \mathrm{~F} & \mathrm{~A}
\end{array}
$$

bp) . . agCGGCTCTGTTAGTCACACTTGTGGGAATTGTGGCATCTGCCCCAATCTCTGACAAACTGGACATTATGAACAAGG

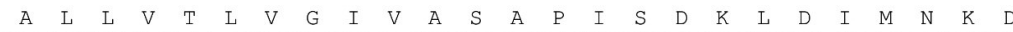
ATACTATTGAACAGGCCCACAGTTTAATCAACAAAATTCTACAAGACATTCCTACAACTCATGCAGCCTGGATAAAGAGCAA $\begin{array}{lllllllllllllllllllllllllllll}T & I & E & Q & A & H & S & L & I & N & K & I & L & Q & D & I & P & T & T & H & A & A & W & I & K & S & K\end{array}$ Ggt... (intron $2 ; 769 \mathrm{bp}$ ) . agAGTCTGACTTTGGGCGACAGCAAGGCTAGgCTGgAGTTGGAGTTCTTGAAGAAAGACA TGTATATACCCTCTGCCCCAGTTCTTCAGCTCATTTCCAATAACTTCAGCATGgt. . (intron 3; 228bp) . agGAAACC $\begin{array}{llllllllllllllllllllll}Y & I & P & S & A & P & V & L & Q & L & I & S & N & N & F & S & M & T\end{array}$ TGCCTTGCAAACATAACGAAGGGACTGCAACTGCATCTCAATCTCTTGAAAGAGATCAGCAATGCAACCACACTGGCTCAGA

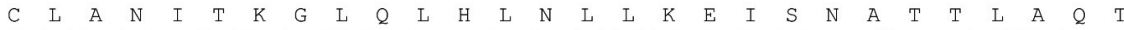

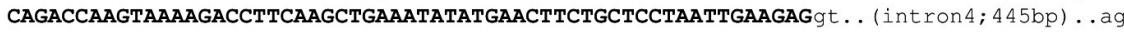

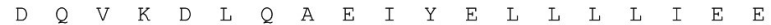
TTGCAGAACCAGGCAGGATTTGACCCATCAAAGCAGGCATCAGATGAGGAGTCGCAGACCCCTGAGCATGATCTGGCCAAGC

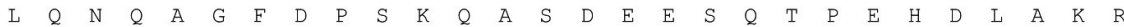
GTCTGACAAATGAATACCTGACCCAGGTGGCAGCCСАCСTCACССTGCAACAGСTTCAGGACTTCAGCTGTGACATCCTCCG

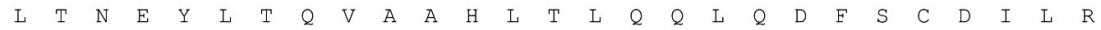
CAGTATTCACAGTATGACCTCCAGCTTGGCAGAGAACCCTAACACTGTGCAGCTCTGCGTAAATAAAGCAGGTCTATGAGCA

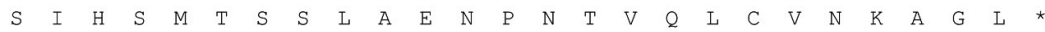
GCCTAGAGTGTAAAGCCCAAAGTATACCTGAGTTTTGACACAAACGCATTGCCTTTCAAAATAAACTTAAAGCCTGCAAAAT TTACTGAAACACATGCAGGTTGACAAGCAAAACTGAAAGTTCCATCTTTGTCTAGTGTCTGTGCTATTCTTCCACAGATGCT AGGAAAATTTGACTTCCCAATGGAAAAAAAAAATAAACCTGGCTGTTGTACTCTATAGAGCATGCATAGATTAAACAGCATT GTACCCTAGCATACATGTCAAAATCAAAGTAAACTTTGGGCTTTACACAAAAACAGATACAGTGACAAGTAAAAAACTAATT AACAGCTGATATGTGTACACAGTCAAATTGTTTGTTGCTGGAATCTTGTTGCACCATCAGTCAGGACATGGAGGCAATGGTA TTTGTTCTTTGTATTTTTCTTCAGATTTGTATTTTATTTATTTATTAATTAGGTTATTTATTTATATTTCACTTGGC АTTTTATTATTATTTAAGTCATTTAGGAACTCCCCTAAATGATTGCATCTTTACAGTCTCATAAAGTAATGTGTTACTTA ATTCACTGTGAAATACTTTACTGTGTCATACAATCTGAATGCATGACCATGTCTCATAACTTGCCAAATGAGAACATGGTCA TTGGATTGATCTCCTGATCTCTGTGAGAACTACACACTGTTTTATTAGTCATTTCATGTGATATTATGAGATATGTAATGT GATATCATACCCATAAAAAGAAGTGAGATTTCAGATGAGTTTACGTATGAATCAGTATTATTTATTTATTTATTAATGTAA CTAAAAATACATGTATTTATTTTTGCCATTATTTGTTATGATATTTTTGGAATATTTGAAGATGTGCCTTATGTTTGATCCA AATAAACATTTCTGATGTGTGTTAAA

Supplementary figure S4.1 Genomic sequences encoding carp G-CSF paralogs. (A) Genomic sequences encoding carp G-CSFa1. LHQP01022752.1, Cyprinus carpio isolate UL-001 Contig22774.

\section{B}

GCTTTGTTACTGCTGGGGTCTGGTGACTTTGTGCATGTTCTTTTTGATTACTGCTGTGATAATGAACTTCCAGggt. . (int M N $\quad \mathrm{F} \quad \mathrm{Q}$ A

ron 1 ; 359bp) . . agCGGCTATATTAGTCACACTTGTGGGAATTGTGGCATCTGCCCCAATCTCTGACAAACTGGACATTTT

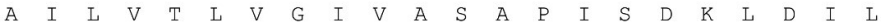
GAACAAGGATACTATTGAACAAGCCCACAGTTTAATCAACAAAATTTTAGAAGATGCTCCTAAAGCTCATGCAGCCTGGATA $\begin{array}{llllllllllllllllllllllllllll}\mathrm{N} & K & \mathrm{D} & \mathrm{T} & \mathrm{I} & \mathrm{E} & \mathrm{Q} & \mathrm{A} & \mathrm{H} & \mathrm{S} & \mathrm{L} & \mathrm{I} & \mathrm{N} & \mathrm{K} & \mathrm{I} & \mathrm{L} & \mathrm{E} & \mathrm{D} & \mathrm{A} & \mathrm{P} & \mathrm{K} & \mathrm{A} & \mathrm{H} & \mathrm{A} & \mathrm{A} & \mathrm{W} & \mathrm{I}\end{array}$ AATAACAAGgt . (intron2; 777bp) .. agGGTCTGACTTTGGGCGACAACACGATTAGACTGCAGCTGAATTACTTGAA

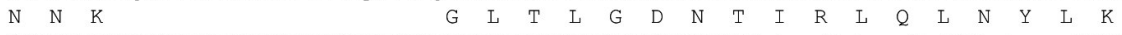
ATCTGTGATACCCTCTGCCCCAGTGCTTCAGAACATCTCCAATATCTCCAGCATGgt. . (intron $3 ; 502 \mathrm{bp}$ ) . . agGAAA $\begin{array}{lllllllllllllllllllllll}S & V & I & P & S & A & P & V & L & Q & N & I & S & N & I & S & S & M & E & \text { I }\end{array}$ CCTGCCTTGCAGACATGGTGAAGGGACTGCAACTTCATCTGAATCTCCTGAATGAGATCATCAAAAAACTGGCCCAGACAGA $\begin{array}{lllllllllllllllllllllllllll}C & L & A & D & M & V & K & G & \text { L } & Q & \text { L } & \text { H } & \text { L } & \text { N } & \text { L } & \text { L } & \text { N } & \text { E } & \text { I } & \text { I } & \text { K } & \text { K } & \text { L } & \text { A } & \text { Q } & \text { T } & \text { D }\end{array}$ CCAAGTGAATGTACTTAAATCTGAAATTCAAGAACTTCATTCCCTAATCAAAAAGgt. . (intron $4 ; 369 \mathrm{bp}$ ) . . agTTGC $\begin{array}{llllllllllllllllllllll}Q & V & N & V & L & K & S & E & I & Q & E & L & H & S & L & I & K & K & & & & \end{array}$ AGAAACAGGCGGGATTTGACCCATCAAAGCATGCAAAAGATGAGCAGTCACAGACCCTTGTGCATGATCTGCACAAGCATCT $\begin{array}{lllllllllllllllllllllllllll}K & Q & A & G & F & D & P & S & K & H & A & K & D & E & Q & S & Q & T & L & V & H & D & L & H & K & H & L\end{array}$ GACAACGGAATTCATGATCCAGGTGGCCGCCCACCTCACCCTGCAACAGCTTCAGGACTTCAGCTGTGACGTCCTCTGCAGT $\begin{array}{llllllllllllllllllllllllllll}T & T & E & E & M & I & Q & V & A & A & H & L & T & L & Q & Q & L & Q & D & F & S & C & D & V & L & C & S\end{array}$ TTTCTTAGTATTCGCAGAATGACCTCCAACATGTCCATGGCAGAGAACCCTAAGACTGTGCAGCTCTGCATAAATGCAGCAG $\begin{array}{lllllllllllllllllllllllllllllllll}F & L & S & I & R & R & M & T & S & N & M & S & M & A & E & N & P & K & T & V & Q & L & C & I & N & A & A & G\end{array}$ GTCTATGAGCAGCGTAGACTGTTAAGCCCAAAGTATACTTGAGTTTTGACACAGAAGCA

$\mathrm{L} *$

Supplementary figure S4.1 Genomic sequences encoding carp G-CSF paralogs. (B) Genomic sequences encoding carp G-CSFa2. LHQP01015831.1, Cyprinus carpio isolate UL-001 Contig15846. 
C

GTGACGCAGCGCTTACGGATGCGGTCTGGGCTTTTTCTCGCTGTTCCTGCAGTGACGAAACAGAAGAATTCCCGGGAGATTI CTCCTTCAGACCCGTCAGATCCGGACCCGAGGGATATTAAACATCTATGACCATACCATTAAATTCGCCCGGAAAATAAGGG GCATTTTGCTGCGGAGCACTIGCTTTGATTCACACAAGAGCAGAACTAAGTTCAAGAAACGTGAAATAATTTCCACTTTTTG GGGCGTTTTCACTCCCCCCCCCCCAAAGAACAGGAGACACAGGAGTGTGTITITCTCCTTGCACCTTTCCTCACTGGTTITT CGCAACGCAGCGTACACGTAATAGATTATAATGTAGTCGCAATAACGAGACAGCTCATCAAAGTTTCCTCAGTTGGACAATC TCAATGGGTTTAAATAGGCTACTAGAATGCAGTAAAATATTCGATACACTCACAACCACAATGAAGTTTTGTCTCAgt. . (I $\begin{array}{lllllll}M & K & F & C & L & I\end{array}$

ntron $1 ; 140 \mathrm{bp}$ ) . . agTTCTTGCAGTGTTGCGCTGCTGTCTGACGCTGGTGGACGCCGCGCCGCTCCAGAAGCAGGAGATG $\begin{array}{llllllllllllllllllllll} & \text { A } & A & V & L & R & C & C & L & T & L & V & D & A & A & P & L & Q & K & Q & E & M\end{array}$ ACGCGCGCTGTGGAGCGCGCCACGAGTTTAGCCAAGAAGATCCTGAGCGACGTTCCCGCGGCGCACCAGGCGTGCGTCAACA $\begin{array}{lllllllllllllllllllllllllllll}T & R & A & V & E & R & A & T & S & L & A & K & K & I & L & S & D & V & P & A & A & H & Q & A & C & V & N & T\end{array}$ cCGCG $g$ t. . (intron $2 ; 220 \mathrm{bp}$ ) . . agGGTTTGGCTCTCTCCAGCGAAGCAGGACACTTGGAGTATTTCTTAAGTGACCTT A G $\quad$ L GGCATCCCCGCGCCGCCGGTGCTCAAGTCAGAGGACCTCAGCATGgt . . (intron $3 ; 420 \mathrm{bp}$ ) . . agGACGTGAGTTTAAG $\begin{array}{llllllllllllllllllllll}G & I & P & A & P & P & V & L & K & S & E & D & L & S & M & D & V & S & L & S\end{array}$ CCGTATTGTGGCCGGTCTGGACCTACACCGTGACCTCCTGCAGGACATCCGAGAGCGCTCGAGCTCCACAGAAGAACTGAGC $\begin{array}{llllllllllllllllllllllllllll}R & I & V & A & G & L & D & L & H & R & D & L & L & Q & D & I & R & E & R & S & S & S & T & E & E & L & S\end{array}$ СTCCTGCTCGCTGATATCACAGACCTGTCTGCTCAGGTCCACCAGgt . . (intron $4 ; 98 \mathrm{bp}$ ) . . agATGCAGCAGCTGGCC

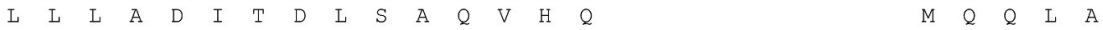
CAGATTCCCAGCACAGTGTCCCAGAAGGCAGCGTTCCCAGCGCTCTCTCCACGGCTCAGCGGTGATTATCAAGTCCAAGTGG

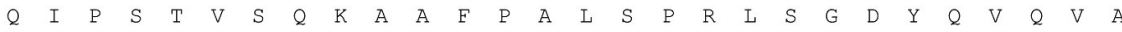
CCATCCATCTTTCTCTTCAGCAGCTGCGCAGCTTCACACAGGACGTCTTCCGCAGTCTGCGCCACATTGCTGCATCAAACTA

$\begin{array}{lllllllllllllllllllllllllllll}I & H & L & S & L & Q & Q & L & R & S & F & T & Q & D & V & F & R & S & L & R & H & I & A & A & S & N & *\end{array}$ GCTGACTTTTCTGTGTGGAGTGTATTTTTGTAAGGCTCACTGGTTGTAAAACATTTGAATGGGTTATCCTTGCTAAGTTTGA GCTGAACTTTGGAATAACTgttgttttaaatggatgtTTTTTATGGTAATTATTAAAGTATTTATTGTTTATTAAATATAT AACTTAAATATTTTTATTGCTGATTTATTTTTATGATGTTATATTGACAGATGTTGTACCCTGTGGGTACTGTGAAGATA TCTATTAAACCTTATTTATTACTGA

Supplementary figure S4.1 Genomic sequences encoding carp G-CSF paralogs. (C) Genomic sequences encoding carp G-CSFb1. LHQP01012478.1, Cyprinus carpio isolate UL-001 Contig12486.

D

TCCGCACGTGTGTGTCAGACAGCAGTTAAACACTACATCATCACATTAATCATCACATTAGTAAAGGGCTITTCGCTGATGA TCGCTTGCTCTGATTCGCGCAAGAGCAGAACTGAGTACAAGAAACGTGAAATAAGGTTTTGGGGCGTTCTCACTTTCCCCCC CACATTTAAAGAACCGGAAACATAGGAGTGTCGTTTTCTCTTTTTTCCCCTATTGGTTTTTCGCAATGCATTGTACGTAATA GGACCACAGTTTAGGTGAAATAGCTAGACAACTCATGGAAGTTTCCCCAGTTGAACAATCTGAACGGGTTTAAATAGCCTGC TAGAAATCCCTTGATCAATGCAGTCAAATATCCCAAACTCTGTGAAGCACAATGCAGCTTTACCTCAgt . . (intron $1 ; 26$

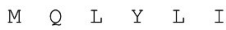

5bp) .. agTTCTCGCAGCGCACTGCTGTCTGACGCTGGTGGACTCCGCGCCGCTCCGGACGCACGAGCTGACGCGCGCCGTG $\begin{array}{lllllllllllllllllllllllllll}I & A & A & H & C & C & L & T & L & V & D & S & A & P & L & R & T & H & E & L & T & R & A & V\end{array}$ GACAGCGCCATGAGTTTAGCCAGGAAGATCCTGAGCGACATTCCCGCAGCGCACGAGGCGTGCGTCAAAGCCACGgt . . (in $\begin{array}{llllllllllllllllllllllllll}D & S & A & M & S & I & A & R & K & I & I & S & D & I & P & A & A & H & E & A & C & V & K & A & T\end{array}$ tron $2 ; 167 \mathrm{bp}$ ) . . agGGTTTGACCCTTTCCAGTGAATCAGAACACTTGGAGTATTTACTGAGTGACATTGGCATCCCTGCG $\begin{array}{llllllllllllllllllllllllllllllll}G & L & T & L & S & S & E & S & E & H & L & E & Y & L & L & S & D & I & G & I & P & A\end{array}$ CCTCCGCTGCTCAAGTCAGAGCACCTCAGCCTGgt . . (intron $3 ; 1591 \mathrm{bp}$ ) . . agGATGTGAGTTTAAGCCGTATTGTAG

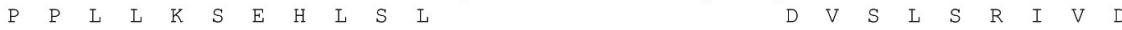
ACGGTTTGGAGCTACACCACAAACTTCTGCAGGAAATTAAAGAGCTCTTGACCTCCACAGAAGAACTGACCCTGCTGCTGGC $\begin{array}{lllllllllllllllllllllllllll}G & L & E & L & H & H & K & L & L & Q & E & I & K & E & L & L & T & S & T & E & E & L & T & L & L & L & A\end{array}$ AGATATTACAGACCTGTCTGCTCAGGTCCATAAGgt. . (intron $4 ; 88 \mathrm{bp}$ ) . . agATGCAGCAGCTGGCCCAGATTCCCAC $\begin{array}{llllllllllllllllllll}D & I & T & D & L & S & A & Q & V & H & K & M & Q & Q & L & A & Q & I & P & T\end{array}$ AGAATCCCAGAAAACCACGTTCCCAGCGATCTCTCCACAGCTCAGCAGTGACTATCATGTCCAAGTGGCCATCCACCTTTGT $\begin{array}{llllllllllllllllllllllllllll}E & S & Q & K & T & T & F & P & A & I & S & P & Q & L & S & S & D & Y & H & V & Q & V & A & I & H & L & C\end{array}$ CTTCAGCAGCTGCGCAGTTTTACTCATGATGTCTTCCGCAGTCTACGCCACATTGCTGCATCAAACTAGTTGACCAGTACTA $\begin{array}{llllllllllllllllllllllllllllllllll}L & Q & Q & L & R & S & F & T & H & D & V & F & R & S & L & R & H & I & A & A & S & N & *\end{array}$ ACCCAACCAACCAGTCCCTGCTATATTTTGTATITTCTGCGTAGAAATGTATTTTTGTAATGCTCACTGTTTGTGTTATCCT TGCTAAGTTTAAGATGAACTTTAGAATAATTTTATTTAATCAGATGTTTTAATTTTAATGTATTTAATATTTA

Supplementary figure S4.1 Genomic sequences encoding carp G-CSF paralogs. (D) Genomic sequences encoding carp G-CSFb2. LHQP01028996.1, Cyprinus carpio isolate UL-001 Contig29026, reverse complement. 

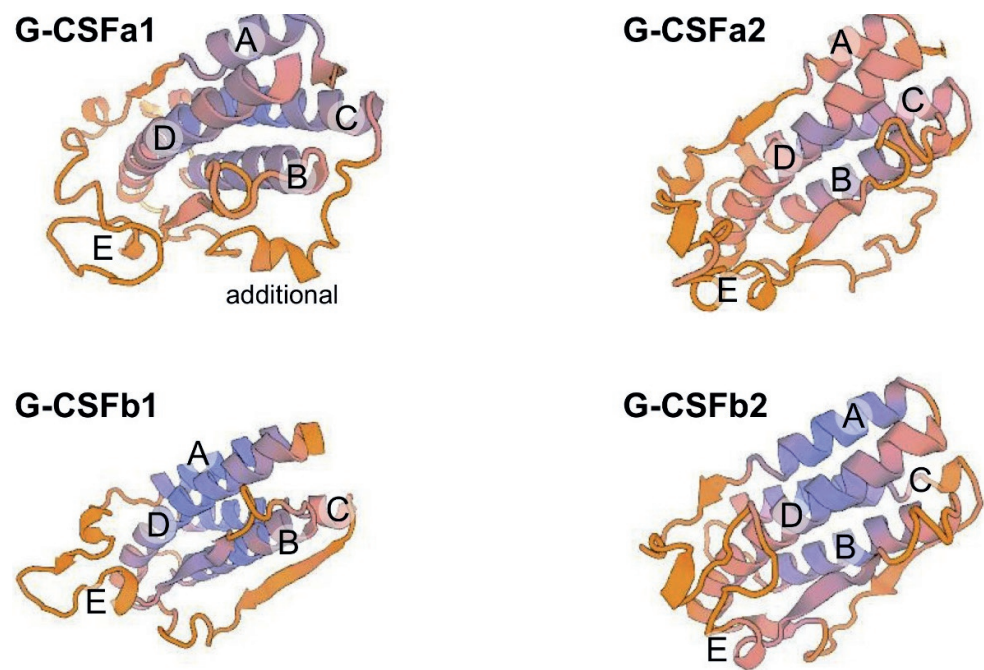

Supplementary figure S4.2 Proposed 3D structure of carp G-CSFa1, G-CSFa2, G-CSFb1 and G-CSFb2. Putative structures are modeled based on the structure of human G-CSF using the SWISSMODEL server (https://swissmodel.expasy.org/).
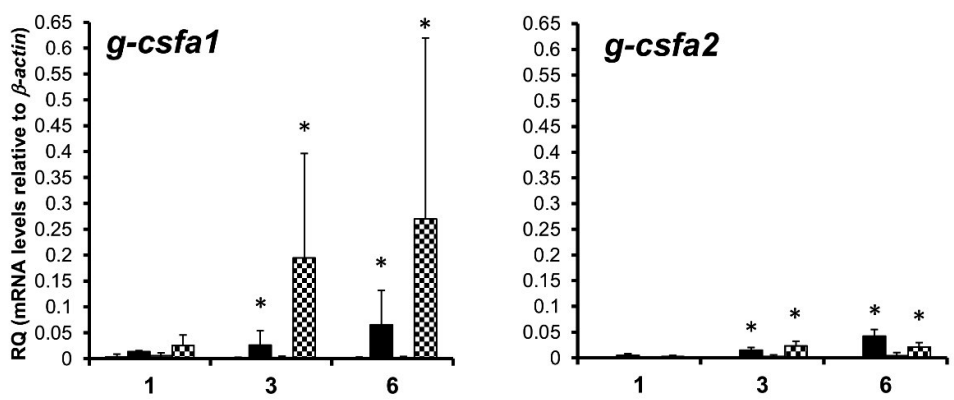

$$
\begin{aligned}
& \text { 口medium } \\
& \text { घPS } \\
& \square \text { polyl:C } \\
& \square \text { ConA/PMA }
\end{aligned}
$$
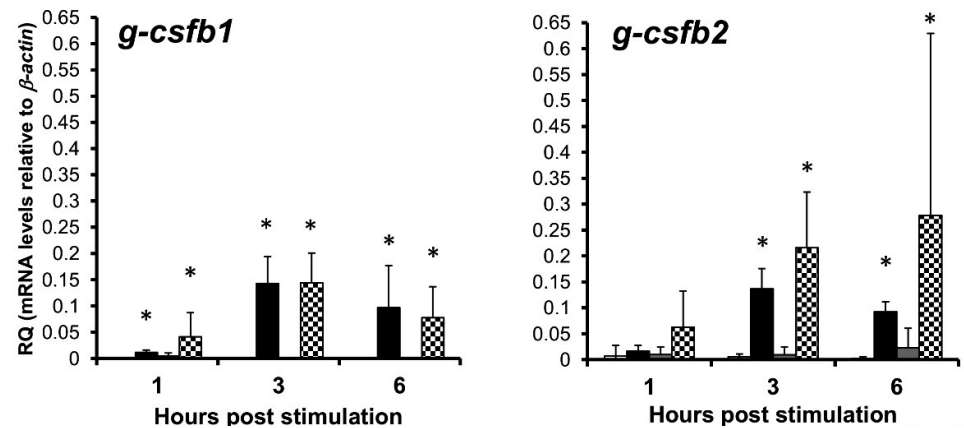

Supplementary figure S4.3 Quantitative mRNA expression analysis of four carp G-CSF paralogs in kidney leukocytes stimulated with mitogens for 1,3 and 6 h. Figure legend on $\mathrm{p} 162$. 
Supplementary figure S4.3 Quantitative mRNA expression analysis of four carp G-CSF paralogs in kidney leukocytes stimulated with mitogens for 1, 3 and $\mathbf{6} \mathbf{h}$. Freshly isolated kidney leukocytes from normal carp were treated with the medium, $50 \mu \mathrm{g} / \mathrm{mL} \mathrm{LPS}, 50 \mu \mathrm{g} / \mathrm{mL}$ polyI:C, or a combination of $10 \mu \mathrm{g} / \mathrm{mL}$ ConA and $1 \mu \mathrm{g} / \mathrm{mL}$ PMA for 1,3 and $6 \mathrm{~h}$. The relative mRNA levels were calculated using $\beta$-actin as reference gene. Data represent mean + standard deviation $(n=3)$. Significant differences compared to the reference sample were determined using one-way ANOVA followed by Dunnet's post hoc test, $(\mathrm{p}<0.05)$ is denoted by $(*)$.
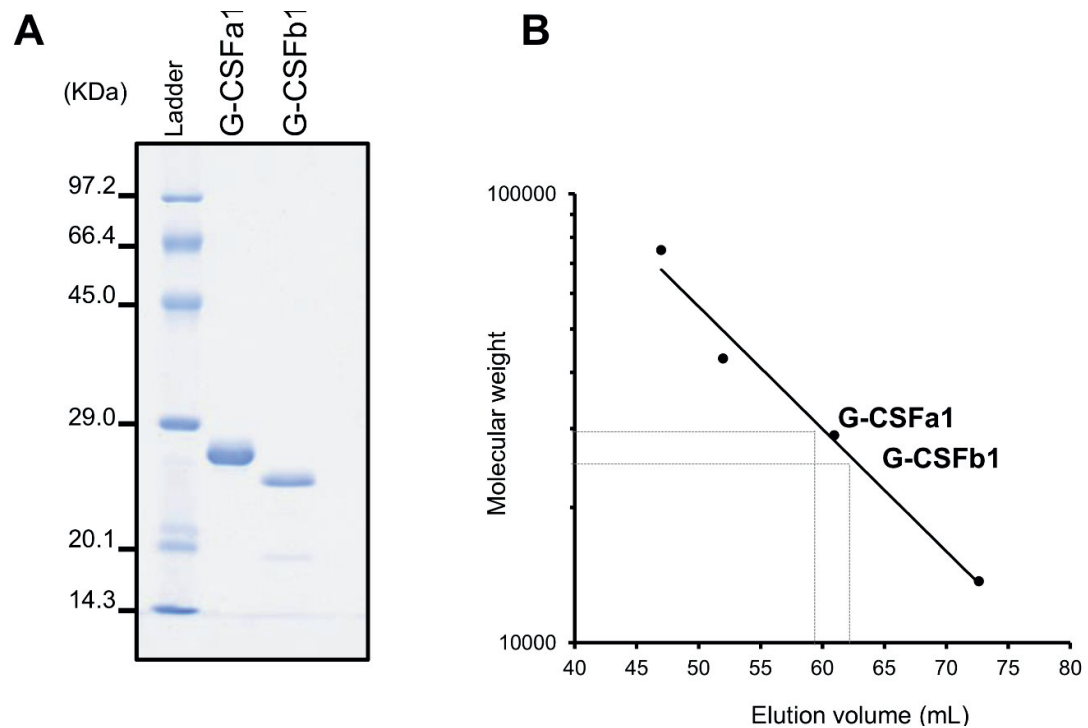

Supplementary figure S4.4. Purification and characterization of recombinant carp G-CSFa1 and G-CSFb1. A) Purified G-CSFa1 and G-CSFb1 were separated on a 12.5\% SDS-polyacrylamide gel under reducing conditions and visualized by staining with Coomassie Brilliant Blue R-250. B) Molecular weight of the recombinant proteins were determined with gel filtration chromatography using a Sephacryl S-100 column (HR 16/60) under native condition. The molecular weights of the standard proteins: conalbumin, 75,000; ovalbumin, 44,000; carbonic anhydrase, 29,000; ribonuclease A, 13,700.

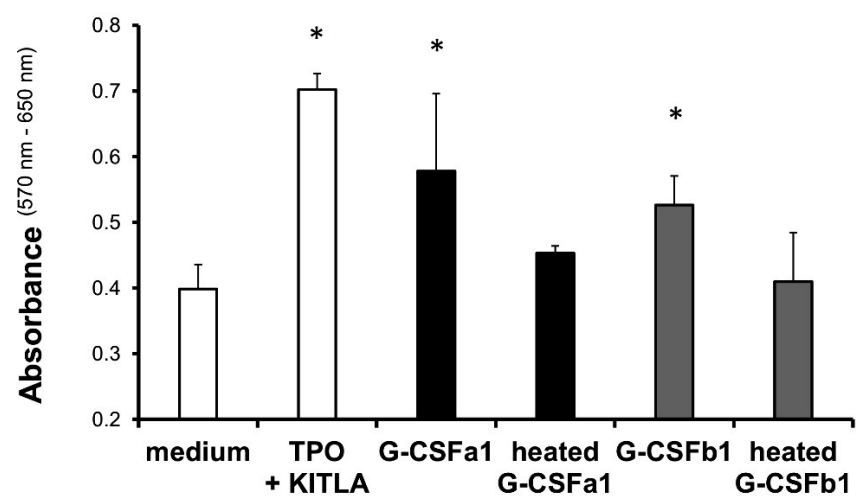


Supplementary figure S4.5 Proliferation of carp kidney neutrophilic granulocyte-like cells. Proliferative response of carp kidney leukocytes $(40,000$ cells) treated with medium alone, recombinant carp TPO (100 ng/mL) plus KITLA (100 ng/mL), recombinant carp G-CSFa1 (100 ng/mL), G-CSFb1 (100 $\mathrm{ng} / \mathrm{mL}$ ) or heat-inactivated G-CSF paralogs. Live cells treated with different stimuli were measured with the MTT assay at day 6 in the culture. Absorbance values at $650 \mathrm{~nm}$ were subtracted from experimental absorbance values at $570 \mathrm{~nm}$ in each well. Data represent mean + standard deviation $(n=3)$. Significant differences compared to the reference (medium) group were determined using one-way ANOVA followed by Dunnet's post hoc test, $(p<0.05)$ is denoted by $\left(^{*}\right)$.

A

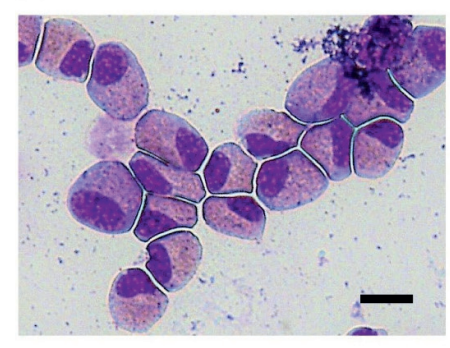

B

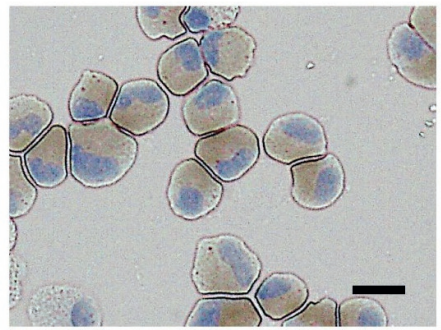

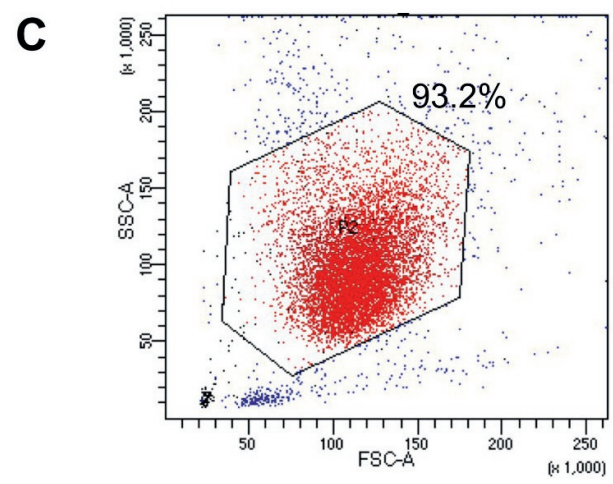

Supplementary figure S4.6 Isolation of kidney neutrophils from carp. A, B) May-Grunwald Giemsa staining 9A) and Peroxidase staining 9B) of isolated kidney neutrophils. Bars indicate $10 \mu \mathrm{m}$. C) Flow cytometric profile of isolated kidney neutrophils. 
2 d before

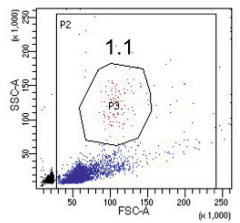

G-CSFa1
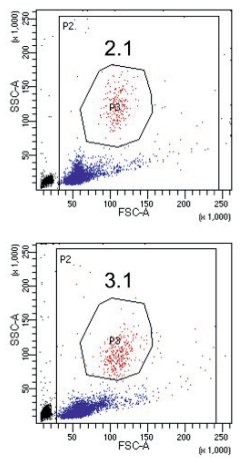

PBS

G-CSFb1

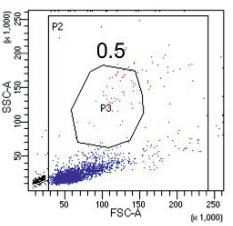

$6 \mathrm{~h}$ after
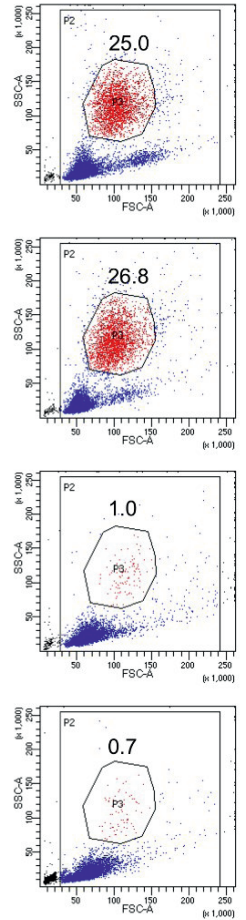

$24 \mathrm{~h}$ after
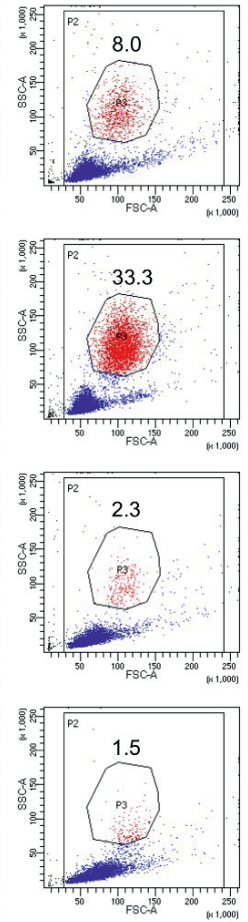

$48 \mathrm{~h}$ after
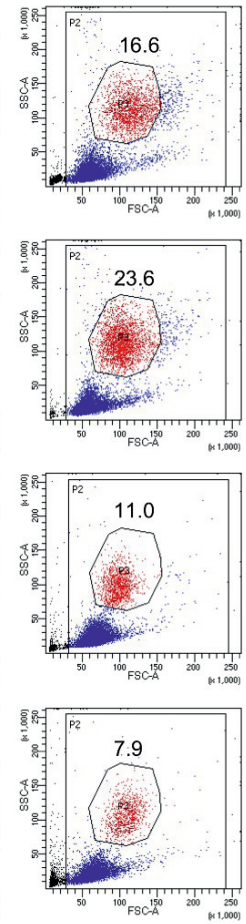

Supplementary figure S4.7 Flow cytometry analysis of peripheral blood leukocytes from carp i.p. injected recombinant G-CSFa1, G-CSFb1 and PBS and uninjected. Peripheral blood leukocytes were collected over time from carp intraperitoneally injected with 1xPBS, recombinant G-CSFa1 and G-CSFb1 and uninjected. Ten thousand leukocytes were analyzed by flow cytometry based on the depiction in forward scatter and side scatter parameters. P3 gates represent the neutrophil population with high side scatter and numbers show percentages of neutrophils in PI- live cells. Representative data in three fish per group are shown. 
Paralogs of carp G-CSF 



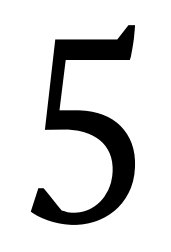

\section{Fish macrophages show distinct metabolic signatures upon uolarization}

Annelieke S. Wentzel ${ }^{1}$, Joëlle J.E. Janssen ${ }^{1,2}$, Vincent C.J. de Boer ${ }^{2}$, Wouter

G. van Veen ${ }^{3}$, Maria Forlenza ${ }^{1}$ and Geert F. Wiegertjes ${ }^{4}$

${ }^{1}$ Cell Biology and Immunology Group, Wageningen University \& Research, Wageningen,

Netherlands

${ }^{2}$ Human and Animal Physiology Group, Wageningen University \& Research, Wageningen, Netherlands

${ }^{3}$ Experimental Zoology Group, Wageningen University \& Research, Wageningen, Netherlands

${ }^{4}$ Aquaculture and Fisheries Group, Wageningen University \& Research, Wageningen,

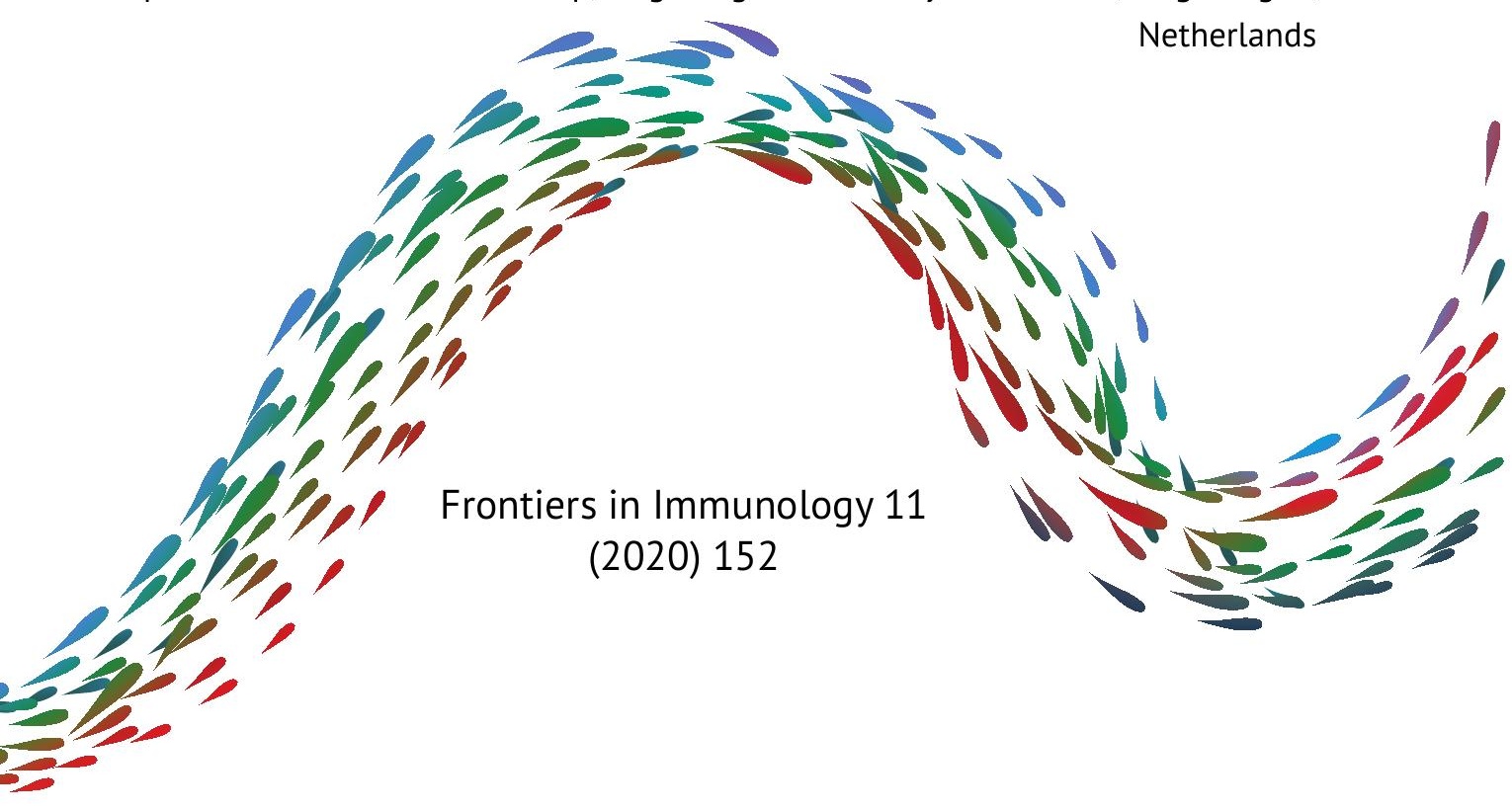




\begin{abstract}
Macrophages play important roles in conditions ranging from host immune defense to tissue regeneration and polarize their functional phenotype accordingly. Next to differences in the use of L-arginine and the production of different cytokines, inflammatory M1 macrophages and anti-inflammatory M2 macrophages are also metabolically distinct. In mammals, M1 macrophages show metabolic reprogramming towards glycolysis while M2 macrophages rely on oxidative phosphorylation to generate energy. The presence of polarized functional immune phenotypes conserved from mammals to fish led us to hypothesize that a similar metabolic reprogramming in polarized macrophages exists in carp. We studied mitochondrial function of M1 and M2 carp macrophages under basal and stressed conditions to determine oxidative capacity by real-time measurements of oxygen consumption and glycolytic capacity by measuring lactate-based acidification. In M1 macrophages, we found increased nitric oxide production and irg1 expression in addition to altered oxidative phosphorylation and glycolysis. In M2 macrophages, we found increased arginase activity and both oxidative phosphorylation and glycolysis were similar to control macrophages. These results indicate that M1 and M2 carp macrophages show distinct metabolic signatures and indicate that metabolic reprogramming may occur in carp M1 macrophages. This immunometabolic reprogramming likely supports the inflammatory phenotype of polarized macrophages in teleost fish such as carp, similar to what has been shown in mammals.
\end{abstract}




\section{Introduction}

Macrophages are essential innate immune cells involved in host defense that play a role in initiating inflammation, but also play a role in the resolution phase of inflammation and in tissue regeneration. These opposing conditions provide microenvironments that drive innate immune cells such as macrophages to display specific effector functions and tailor immune response to either combat pathogens or repair damage. In mammals, depending on the exact microenvironment, an array of different macrophage phenotypes can exist with the most polarized phenotypes termed M1 and M2 [1]. Inflammatory macrophages are commonly associated with $\mathrm{T}$ helper-1 responses (hence M1) and produce pro-inflammatory cytokines, antimicrobial nitric oxide (NO) or other reactive oxygen radicals (ROS) [2-4]. Antiinflammatory macrophages are commonly associated with $\mathrm{T}$ helper-2 responses (hence M2), produce anti-inflammatory cytokines and show increased arginase activity. Hence, M1 macrophages metabolize the amino acid L-arginine to produce NO, while M2 macrophages metabolize the same substrate to produce proline and polyamines [2]. Thus, M1 and M2 macrophages show opposing metabolism of L-arginine.

In mammals, macrophages are also metabolically reprogrammed to enhance opposing pathways to generate energy upon polarization (reviewed by [5, 6]. Most studies addressing macrophage immunometabolism have been performed in mice. IL-4-activated M2 macrophages rely primarily on oxidative phosphorylation (OXPHOS) for energy production, with the exact role of fatty acid oxidation still being debated [6]. In contrast, upon activation with bacterial lipopolysaccharide (LPS) alone or in combination with IFN- $\gamma$, M1 macrophages show metabolic reprogramming from OXPHOS towards glycolysis. Reprogramming of M1 macrophages towards glycolysis is accompanied by two 'breaks' in the tricarboxylic acid cycle (TCA cycle) and inhibition of parts of the electron transport chain (ETC) in the mitochondria [5] (Figure 5.1). The two breaks in the TCA cycle are due to lower activity and expression of isocitrate dehydrogenase and succinate dehydrogenase (SDH) and lead to an accumulation of citrate and succinate (Figure 5.1), which supports important pro-inflammatory immune functions of M1 macrophages. For example, accumulated citrate is shuttled out of the mitochondria and subsequent accumulation in the cytosol contributes to the production of NO, ROS and fatty acid synthesis for membrane and granule formation. Accumulated succinate contributes to ROS production and can stabilize hypoxia-inducible factor 1-alpha 
(HIF1 $\alpha$ ), which activates the glycolytic pathway and drives inflammation through increased expression of IL-1 $\beta$ [7]. Released succinate acts as an alarmin in the extracellular microenvironment and is recycled to generate a feed-forward loop, further increasing IL-1 $\beta$ production [8]. Last but not least, inhibition of the ETC is mediated both by NO and itaconate (Figure 5.1). Itaconate, produced from citrate with the enzyme encoded by irg1, is considered an important regulator of the metabolic reprogramming as it inhibits both ETC and TCA-cycle through (SDH), but is also important to dampen inflammatory functions at later timepoints [6, 9]. Therefore, metabolic reprogramming from oxidative metabolism to glycolysis supports several inflammatory immune functions in M1 macrophages.

Fish macrophages show several of the immune functions typically associated with M1 and M2 macrophages and thus macrophage polarization may be largely conserved [10-12]. For example, M1 macrophages of carp show increased NO production after stimulation with LPS alone [13, 14] or in combination with Ifn- $\gamma$ [15] and show increased expression of il-1 $\beta$ $[10,14]$. Zebrafish macrophages show stabilization of Hif1 $\alpha$ and

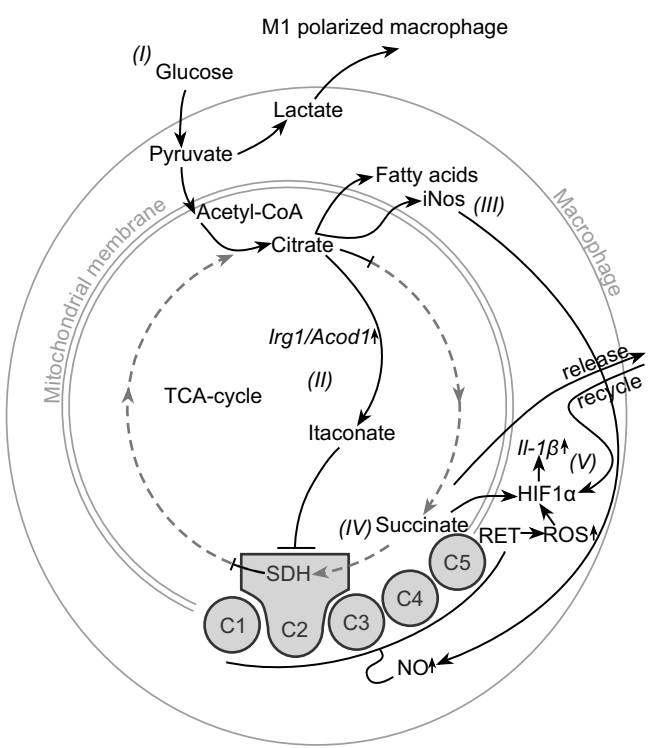

Figure 5.1 Schematic representation of metabolic reprogramming towards glycolysis in M1 macrophages.

Pathways indicated in black are retained or enhanced in M1, pathways indicated in (dashed) grey and are reduced in M1. The uptake of glucose and glycolysis (I) is increased in M1 which results in excess pyruvate that is converted to lactate and released from the cell. In the mitochondria, two breaks in the TCA cycle result in the accumulation of citrate and succinate. Accumulation of citrate contributes to three important changes; 1) Citrate is converted to itaconate by the enzyme encoded by Irg1 (II). Itaconate in turn can inhibit both the TCA cycle and the electron transport chain by blocking succinate dehydrogenase (SDH). 2) Citrate is also shuttled out of the mitochondria where it promotes NO production and fatty acid synthesis (III). 3) Oxidative phosphorylation through the electron transport chain is inhibited, both by itaconatemediated inhibition of SDH and by increased production of NO (IV). This causes increased levels of succinate which stabilize hypoxia-inducible factor 1-alpha (HIF1 $\alpha$ ) and are also released and recycled by the cell. Reactive oxygen species (ROS) generated by reverse electron transport (RET) over the hyperpolarized mitochondrial membrane also stabilize HIF $1 \alpha$ which in turn results in increased Il$1 \beta$ expression (V). Figure based on Van den Bossche et al., 2017. 
il-1 $\beta$ expression following mycobacterial infection [16, 17]. M2 macrophages of carp and goldfish show increased arginase activity after stimulation with cAMP or Il-4 [10, 18]. The apparent conservation of macrophage polarization led us to hypothesize a conservation of the underlying changes in energy metabolism and immunometabolic reprogramming in fish macrophages. We therefore studied mitochondrial function of M1 and M2 polarized carp macrophages under basal and stressed conditions. We determined oxidative capacity by real-time measurements of oxygen consumption, and we measured glycolytic capacity by measuring lactatebased acidification. Our data provide the first evidence that carp macrophages can use different pathways for energy metabolism associated with macrophage polarization in teleost fish. We discuss the implications of our findings for studying macrophage polarization in exothermic aquatic vertebrates.

\section{Materials and methods}

\section{Animals}

European common carp (Cyprinus carpio carpio L.) used for experiments were the offspring of a cross between the R3 strain of Polish origin and the R8 strain of Hungarian origin [19]. Carp were bred and reared in the aquatic research facility of Wageningen University and Research at $20-23^{\circ} \mathrm{C}$ in recirculating UV-treated tap water and fed pelleted dry food (Skretting, Nutreco) twice daily. All experiments were performed with the approval of the Animal Experiments Committee of Wageningen University and Research (Ethical Committee documentation number 2017.W-0034).

\section{In vitro culture and polarization of head kidney-derived carp macrophages}

The head kidney in teleost fish is a primary hematopoietic organ and can be considered the functional equivalent of bone marrow [20]. Head kidney-derived macrophages (hereon referred to as macrophages) were obtained as previously described [10]. After six days of culture at $27^{\circ} \mathrm{C}$, macrophages were polarized to M1 or M2 state. In short, macrophages were harvested by gentle scraping after incubation on ice for 15 minutes. Cells were pelleted at $450 \mathrm{x}$ g for $10 \mathrm{~min}$ at $4^{\circ} \mathrm{C}$ before resuspension in cRPMI+ (RPMI 1640 culture medium with 25 mM HEPES and $2 \mathrm{mM} \mathrm{L}$-glutamine (12-115F, Lonza), supplemented with L-glutamine (2 $\mathrm{mM}$, Gibco), penicillin $\mathrm{G}(100 \mathrm{U} / \mathrm{ml})$, streptomycin sulfate $(100 \mu \mathrm{g} / \mathrm{ml}$, Gibco) and heat-inactivated pooled carp serum $(1.5 \% \mathrm{v} / \mathrm{v}))$. Cells were cultured at $27^{\circ} \mathrm{C}$ in the 
presence of $5 \% \mathrm{CO}_{2}$ in cRPMI+ unless indicated otherwise. Macrophages stimulated for $24 \mathrm{~h}$ with 20 or $50 \mu \mathrm{g} / \mathrm{ml}$ LPS (Escherichia coli, L2880, Sigma-Aldrich) were considered M1. Macrophages stimulated for $24 \mathrm{~h}$ with $0.5 \mathrm{mg} / \mathrm{ml}$ dibutyryl cAMP (N $\mathrm{N}^{6}, 2^{\prime}$-O-dibutryladenosine 3':5'-cyclic monophosphate sodium D0627, Sigma Aldrich, abbreviated as cAMP) were considered M2.

\section{Nitric oxide production}

Nitric oxide (NO) production for confirmation of functional polarization was determined in culture supernatants of polarized macrophages. In brief, $5 \times 10^{5}$ macrophages per well were seeded in 96-wells plates (Corning) in $150 \mu \mathrm{l}$ of cRPMI+. After polarization, NO production was determined as nitrite in $75 \mu \mathrm{l}$ culture supernatant as described previously [21] and expressed in $\mu \mathrm{M}$ using a nitrite standard curve.

\section{Arginase activity}

Arginase enzymatic activity for confirmation of functional polarization into M2 was measured in cell lysates and normalized using a ratio of the sample protein content compared to lysate of control cells. A total of $1.5 \times 10^{6}$ cells polarized for $24 \mathrm{~h}$ in $450 \mu \mathrm{l} \mathrm{cRPMI+}$, were lysed in $100 \mu \mathrm{l}$ of $0.1 \%$ Triton X-100. Protein content of the samples was determined using the Bradford protein dye reagent (Bio-Rad) according to the manufacturers protocol. Arginase activity was measured in $25 \mu \mathrm{l}$ lysate essentially as described previously for $50 \mu \mathrm{l}$ lysate [10], but volumes were scaled down accordingly. Arginase activity was determined as the conversion of $\mathrm{L}$-arginine to urea by arginase and expressed in $\mathrm{nmol} / \mathrm{min} / 10^{6}$ cells.

\section{Extracellular lactate}

The release of lactate into the culture supernatant was measured using a lactate colorimetric assay (Kit II K627, BioVision) in filtered samples (Amicon 10K spin column; Z677108-96EA; Sigma-Aldrich) according to the manufacturer's instructions. Briefly, $1.5 \times 10^{6}$ cells were polarized in $450 \mu \mathrm{l}$ cRPMI+ before culture supernatants from triplicate wells were pooled and filtered. Fifty $\mu \mathrm{l}$ of $25 \mathrm{x}$ diluted culture supernatant was combined with $50 \mu \mathrm{l}$ reaction mix in a 96-well plate and incubated for $30 \mathrm{~min}$ at room temperature. OD was measured at $450 \mathrm{~nm}$ and the concentration of lactate present in culture supernatants was calculated based on a calibration curve supplied by the manufacturer. 


\section{Mito Stress test}

Extracellular flux analysis of polarized macrophages was performed by measuring oxygen consumption rate (OCR) and extracellular acidification rate (ECAR) using a Seahorse XFe96 extracellular flux analyzer (Agilent). We essentially applied the manufacturer's protocol and optimized culture conditions, cell density and carbonyl cyanide-4 (trifluoromethoxy) phenylhydrazone (FCCP) concentrations to measure OCR and ECAR in carp macrophages and adjusted all incubation steps in the protocol to $27^{\circ} \mathrm{C}$. For this, the XFe96 analyzer was kept at room temperature and set to $20^{\circ} \mathrm{C}$, which would keep the analyzer at a stable $27^{\circ} \mathrm{C} \pm 1^{\circ} \mathrm{C}$ during the complete assay.

To measure OCR and ECAR, culture medium of $1 \times 10^{5}$ macrophages/well polarized for $24 \mathrm{~h}$ in XF96 V3 PS Cell Culture Microplates (Agilent) was replaced with 180 $\mu \mathrm{l}$ non-buffered Seahorse XF base medium supplemented with $10 \mathrm{mM}$ D-glucose (Sigma) and $4 \mathrm{mM} \mathrm{L-glutamine} \mathrm{(Gibco)} \mathrm{at} \mathrm{pH}$ 7.4. After incubation without $\mathrm{CO}_{2}$ for $45 \mathrm{~min}$ at $27^{\circ} \mathrm{C}$, OCR and ECAR were measured at basal level and after subsequent addition of $1.5 \mu \mathrm{M}$ oligomycin, $0.2 \mu \mathrm{M}$ (FCCP) and $2.5 \mu \mathrm{M}$ antimycin $\mathrm{A} / 1.25 \mu \mathrm{M}$ Rotenone $/ 40 \mu \mathrm{M}$ Hoechst DNA stain (all from Sigma). The standard 20 min equilibration cycle at the beginning of a Seahorse run was replaced by an incubation for $10 \mathrm{~min}$ without additional mixing before measurements were started. Measurement cycles consisted of 1 min mixing, 1 min waiting and 3 min measuring. A minimum of 4 technical replicates was used for each condition.

To normalize OCR and ECAR measurements, we determined the area covered with Hoechst stained nuclei for each well according the manufacturer's instructions. We subsequently used the ratio for each well compared to the average of all controls for normalization of the OCR and ECAR data. Images were taken with a Cytation 1 plate reader (BioTek) and analyzed using CellProfiler (Version 3.1.9).

\section{Real time activation of macrophages}

To track glycolysis and oxidative metabolism during activation of macrophages in real time, $1 \times 10^{5}$ macrophages/well were plated in XF96 V3 cell culture plates and cultured overnight. The cell culture medium was replaced with $180 \mu$ l Seahorse XF RPMI medium with $10 \mathrm{mM} \mathrm{D}$-glucose and $4 \mathrm{mM}$ L-glutamine (pH 7.4). After incubation without $\mathrm{CO}_{2}$ for $45 \mathrm{~min}$, OCR and ECAR were recorded at basal level and for at least $4 \mathrm{~h}$ after addition of 20 or $50 \mu \mathrm{g} / \mathrm{ml} \mathrm{LPS,} 0.5 \mathrm{mg} / \mathrm{ml}$ cAMP or medium 
as unstimulated controls as the first injection in the Seahorse run. The standard 20 min equilibration cycle at the beginning of a Seahorse run was replaced by an incubation for $10 \mathrm{~min}$ without additional mixing. Measurement cycles consist of $30 \mathrm{sec}$ mixing, $1.5 \mathrm{~min}$ waiting and 3 min measuring. A minimum of 4 technical replicated was used for each condition.

\section{Gene expression analysis of $\operatorname{irg} 1$}

Transcriptome sequencing was performed as described previously [22, 23]. After reads were aligned to the latest genome assembly of common carp (BioProject: PRJNA73579) [22], differential gene expression was analyzed using the bioinformatics package DESeq 2.0 (v1.22.2) and R statistical software (3.5.5) [24] as described before [23]. Statistical analysis was performed using a paired design with unstimulated cells as control and performed for LPS (30 $\mu \mathrm{g} / \mathrm{ml})$ and cAMP $(0.5 \mathrm{mg} /$ $\mathrm{ml}$ ) stimulated macrophages independently ( $n=3$ independent cultures for each stimulus).

\section{Statistics}

The mean of technical replicates was used for paired statistical analysis of $n=6$ biological replicates (NO production, arginase activity and Mito Stress test), $\mathrm{n}=5$ biological replicates (lactate assay) or $\mathrm{n}=3$ biological replicates (gene expression).

Analysis of NO, arginase assays and lactate assays was performed with a repeated measures ANOVA followed by Tukey's post hoc tests to determine significant differences between treatments. Normal distributions were confirmed (Shapiro-Wilk test) and in absence of sphericity (Mauchly's test of sphericity), the GreenhouseGeisser correction was applied. For Mito Stress test analysis, the Friedman's 2-way ANOVA by ranks was used followed by Dunns post-hoc tests for the non-normally distributed samples. Statistical analysis was performed using IBM SPSS statistics Version 26 and GraphPad Prism 5. Gene expression analysis was performed using DESeq2 as described above. Differences were considered significant when $p<0.05$. 


\section{Results}

\section{Metabolic signatures of polarized carp macrophages}

Macrophages were confirmed as polarized prior to determining their metabolic pathways. LPS-stimulated M1 macrophages showed increased NO production compared to unstimulated macrophages while cAMP-stimulated macrophages did not (Figure 5.2A). cAMP-stimulated M2 macrophages showed increased arginase activity compared to unstimulated macrophages while LPS-stimulated macrophages did not (Figure 5.2B).

Metabolic signatures of polarized carp macrophages were examined by measuring extracellular lactate production, expression of irg1 and accumulation of intracellular citrate and succinate. All these parameters were shown to play a role in the metabolic reprogramming of murine M1 macrophages from OXPHOS towards glycolysis. In carp macrophages, increased lactate concentrations were measured in culture supernatants of M1 but not M2 macrophages compared to unstimulated macrophages (Figure 5.2C). Also, gene expression of irg1 was increased to a
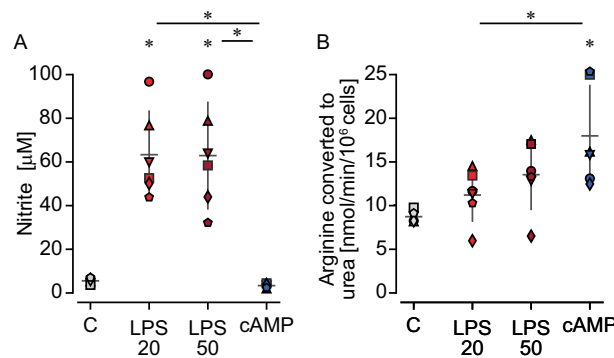

Figure 5.2 Polarized macrophages of carp show indications of distinct metabolic profiles. Carp macrophages were left unstimulated (grey, control) or were polarized for $24 \mathrm{~h}$ with LPS (red; 20 or $50 \mu \mathrm{g} / \mathrm{ml}$ ), or with cyclic AMP (blue; 0.5 $\mathrm{mg} / \mathrm{ml}$ ). A) Nitric oxide production measured as nitrite concentration $(\mu \mathrm{M})$. B) Arginase activity measured as conversion of L-arginine to urea by arginase (nmol/min $/ 10^{6}$ cells). C) Lactate concentration $(\mathrm{mM})$. Shown are individual fish (each indicated by a unique symbol) and the mean

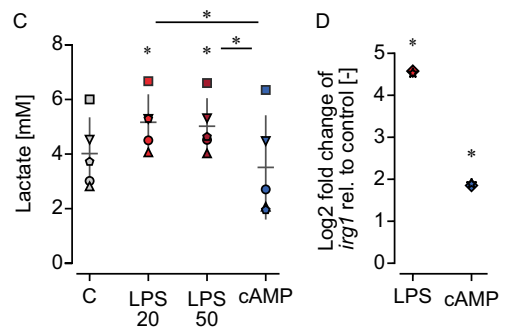
and standard deviation of $n=6(A$ and $B)$ or $n=$ 5 (C) biological replicates. D) Gene expression of two carp irg1 paralogs (cypCar_00026281 (star) and cypCar_00007903 (diamond)) stimulated for 6 $\mathrm{h}$ with $30 \mu \mathrm{g} / \mathrm{ml}$ LPS (red) or with $0.5 \mu \mathrm{g} / \mathrm{ml}$ cAMP (blue) analyzed by DESeq2 after transcriptome sequencing. Gene expression data are shown as $\log 2$ fold change compared to unstimulated controls ( $\mathrm{n}=3$ biological replicates). Data (AC) were analyzed using a repeated measures ANOVA with Geisser-Greenhouse correction followed by Tukey's post-hoc tests. Data (D) were analyzed by DESeq2 as part of a transcriptional study. Differences were considered significant when $\mathrm{p}<0.05$. Asterisks $(*)$ indicate significant differences between stimulated and control groups or between groups (line with asterisk). Since there were no clear differences between the two concentrations of LPS, experiments were continued with $20 \mathrm{mg} / \mathrm{ml}$ LPS. 
much higher extent in M1 than M2 macrophages (Figure 5.2D). Accumulation of intracellular citrate did not show differences between M1 and M2 macrophages, whereas intracellular succinate could not be quantified because levels were below detection limit (data not shown). Overall, the combination of increased lactate production and increased irg1 expression indicated that carp M1 macrophages showed a metabolic reprogramming towards glycolysis.

\section{Oxygen consumption rate (OCR) and extracellular acidification rate (ECAR) of polarized carp macrophages}

To study in detail mitochondrial function and oxidative capacity in polarized carp macrophages, we first optimized the Seahorse Mito Stress test for use with carp macrophages at lower $\left(27^{\circ} \mathrm{C}\right)$ temperature. We optimized cell density to $1 \times 10^{5}$ cells/well and found carp macrophages to be particularly sensitive to FCCP with a relatively low optimum concentration of $0.2 \mu \mathrm{M}$ (tested range $0.1-3 \mu \mathrm{M}$ ). Then, we determined oxygen consumption rate (OCR) (Figure 5.3A) as a measure for oxidative metabolism and extracellular acidification rate (ECAR) (Figure 5.3B) as a measure for glycolysis. M1 and M2 macrophages did not show clear differences in OCR or ECAR at basal level (time range $a$ ). Injection of oligomycin blocks complex $\mathrm{V}$ of the electron transport chain and as such inhibits ATP production. Both M1 and M2 macrophages therefore decreased oxygen consumption while increasing extracellular acidification (time range $b$ ). Disruption of the mitochondrial membrane potential by injection of FCCP induces maximal oxygen consumption. Indeed, after FCCP injection M1 and M2 macrophages both increased oxygen consumption, but M1 macrophages clearly showed much lower OCR than control or M2 macrophages (time range $c$ ). Finally, injection of antimycin A and rotenone inhibits complex IIV and complex I of the ETC, thereby completely blocking the ETC. M1 and M2 macrophages did not show differences in non-mitochondrial respiration after antimycin A and rotenone were injected (time range $d$ ).

Oxygen consumption and extracellular acidification data were used to quantify different metabolic parameters. Basal respiration and ATP-linked respiration (OCR used for ATP synthesis) were not significantly different between control and polarized carp macrophages. However, spare respiratory capacity after injection with FCCP (time range $c$ ), was significantly impaired in M1 carp macrophages, which reflected the impaired capacity of M1 macrophages to increase respiration and meet increased energy demands when stressed. Maximal respiration was therefore 
also significantly reduced in M1 carp macrophages (Figure 5.4A). In contrast, basal acidification or glycolytic reserve did not change with polarization (Figure 5.4B) indicating that the above-discussed reduction in oxidative capacity of M1 was not mirrored by an increase in glycolysis. Taking all parameters together, polarized M1 macrophages of carp clearly show a different metabolic profile compared to control and M2 macrophages (Figure 5.4C).

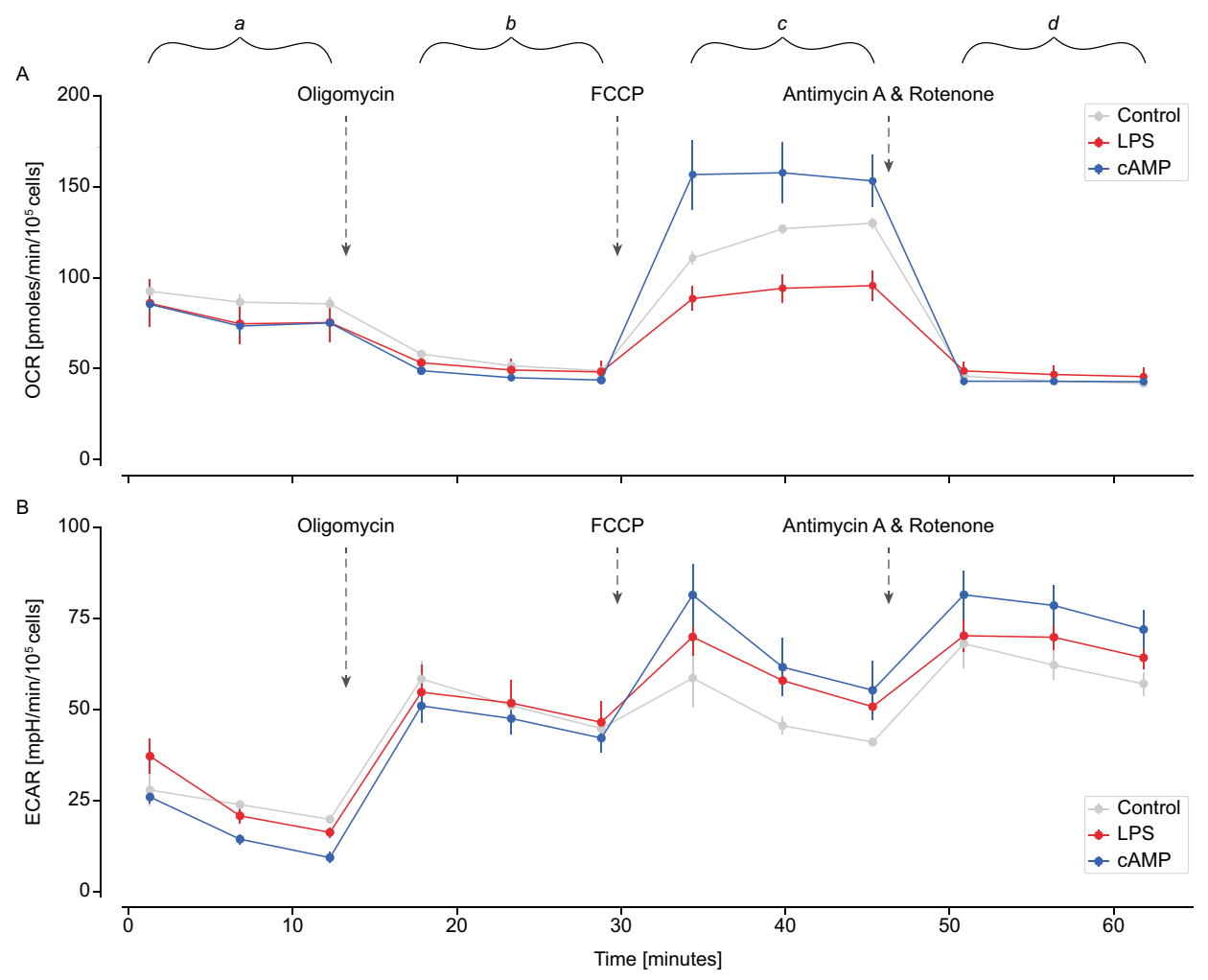

Figure 5.3 Oxygen consumption rates (OCR) and extracellular acidification rate (ECAR) of polarized carp macrophages. Carp macrophages were left unstimulated (grey, control) or were polarized for $24 \mathrm{~h}$ with LPS (red; $20 \mu \mathrm{g} / \mathrm{ml}$ ), or with cyclic AMP (blue; $0.5 \mu \mathrm{g} / \mathrm{ml}$ ). Graphs display Mito Stress test profiles of A) oxygen consumption rate (OCR) and B) extracellular acidification rate (ECAR) at basal level (time range a) and after subsequent addition of oligomycin (time range b), carbonyl cyanide-4 (trifluoromethoxy) phenylhydrazone (FCCP) (time range c) and antimycin/rotenone (time range d). Normalized rates are shown as mean and SEM of one representative experiment out $n=6$ biological replicates. 

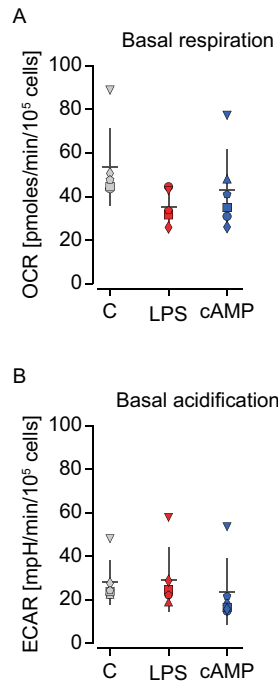
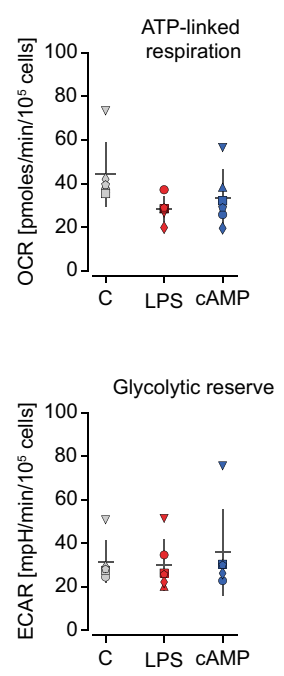
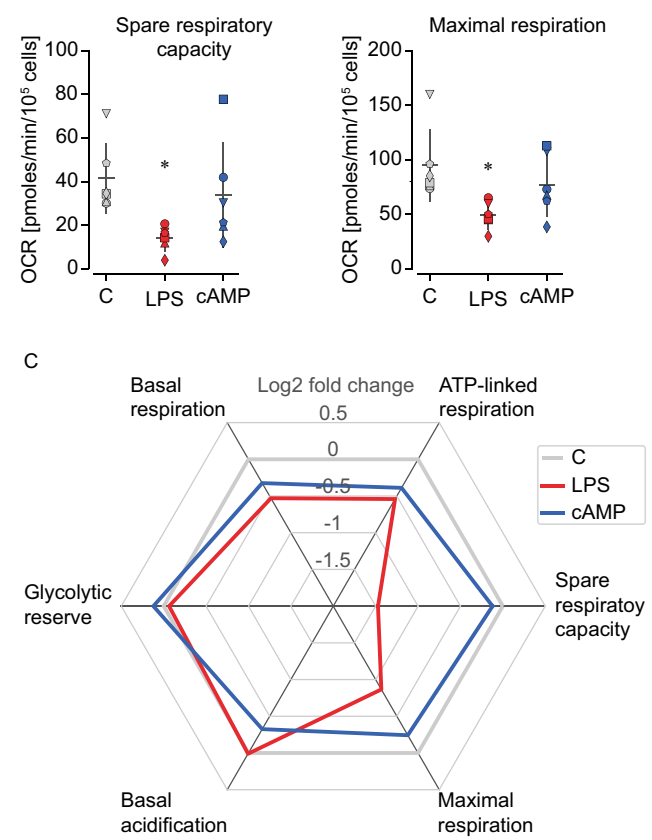

Figure 5.4 Metabolic parameters underline differences in oxidative potential between polarized carp macrophages. Carp macrophages were left unstimulated (grey, control) or were polarized for $24 \mathrm{~h}$ with LPS (red; $20 \mu \mathrm{g} / \mathrm{ml}$ ), or with cyclic AMP (blue; $0.5 \mathrm{mg} / \mathrm{ml}$ ). A) Oxidative parameters based on oxygen consumption rate (OCR) include basal respiration $\left(\mathrm{OCR}_{\mathrm{a}}-\mathrm{OCR_{ \textrm {d } }}\right)$, oxygen used for ATP synthesis $\left(\mathrm{OCR}_{\mathrm{a}}\right.$ - $\left.O C R_{b}\right)$, maximal respiration $\left(O C R_{c}-O C R_{d}\right)$ and spare respiratory capacity $\left(O C R_{c}-O C R_{a}\right)$. B) Glycolytic parameters based on extracellular acidification rate (ECAR) include basal acidification rate $\left(E^{2} \mathrm{AR}_{\mathrm{a}}\right)$ and glycolytic reserve $\left(\mathrm{ECAR}_{\mathrm{b}}-\mathrm{ECAR}_{\mathrm{a}}\right)$. C) Spider plot depicting both, oxidative and glycolytic parameters of polarized carp macrophages (mean log2 fold change compared to respective controls). Metabolic parameters were calculated from normalized Mito Stress test profiles of polarized macrophages and based on the mean of three consecutive measurements as indicated in figure 5.3 with time periods a, $\mathrm{b}, \mathrm{c}$ or d. Normalized rates are shown for individual fish (each indicated by a unique icon) and the mean and SD of $\mathrm{n}=6$ biological replicates. Differences were considered significant when $p<0.05$. Asterisks (*) indicate significant differences between stimulated and control groups.

Although lactate levels were increased in M1 macrophage culture supernatants (see Figure 5.2C), polarized carp macrophages did not show differences in basal extracellular acidification rates (ECAR) nor in glycolytic reserve after $24 \mathrm{~h}$ of polarization (see Figures 5.3B and 5.4B). This could indicate that ECAR normalized after $24 \mathrm{~h}$ to control ECAR levels, and that ECAR peaked at earlier timepoints than $24 \mathrm{~h}$. We thus performed a preliminary real-time measurement of OCR and ECAR before, during and immediately after activation of carp macrophages. We observed a rapid, dose-dependent increase in ECAR that remained high for the duration of the experiment, but only in M1 macrophages (LPS stimulation) (Figure 5.5B). In 
contrast, M2 macrophages (cAMP stimulation) showed a rapid but very short increase in ECAR which rapidly returned to values below controls. No differences in OCR were observed within this time frame (Figure 5.5A). These results suggest that M1 carp rapidly increase their basal glycolysis and that this increase is sustained up to 4 hours but reverts to basal levels at $24 \mathrm{~h}$.

\section{Discussion}

Previous studies have shown a general conservation of carp macrophage immune function with respect to their ability to polarize towards a pro- or anti-inflammatory profile in response to conventional M1 or M2 stimuli. These observations led us to hypothesize the occurrence of metabolic reprogramming of polarized macrophages of carp. To study this hypothesis, we determined oxidative and glycolytic capacity of M1 and M2 carp macrophages by measuring oxygen consumption rates (OCR) and extracellular acidification rates (ECAR) under basal and stressed conditions in real-time. Carp M1 macrophages show i) reduced maximal respiration and ii) reduced spare respiratory capacity, both indicative of a reduction in oxidative capacity. Furthermore, carp

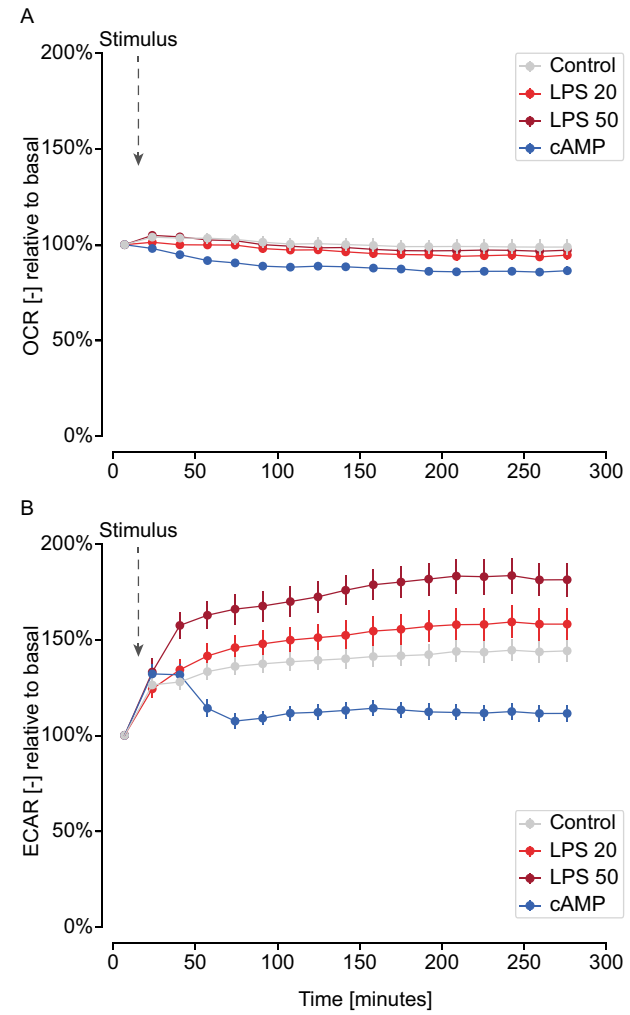

Figure 5.5 Real time measurements of extracellular acidification rate (ECAR) after activation with LPS or cyclic AMP. Carp macrophages were left unstimulated (grey, control) or were stimulated with LPS (red; 20 $\mu \mathrm{g} / \mathrm{ml}$ or $50 \mu \mathrm{g} / \mathrm{ml}$ ), or with cyclic AMP (blue; $0.5 \mathrm{mg} / \mathrm{ml}$ ) by injection after determining basal OCR and ECAR levels. Panels represent real time measurements of OCR (A) and ECAR (B) for one representative fish out of two. Means of three consecutive OCR and ECAR measurements were normalized to basal level for each well using the mean of three basal measurements before injection of the stimulus. Markers represents the mean and SEM of technical replicates expressed relative to basal rates. M1 macrophages show iii) increased lactate production after activation with LPS and a rapid increase in ECAR which is sustained up to $4 \mathrm{~h}$ but not $24 \mathrm{~h}$. Finally, carp macrophages show iv) increased production of nitric oxide (NO) and show v) increased gene expression of irg1, which encodes an enzyme that converts citrate 
to itaconate. Itaconate is a metabolite that can inhibit both the TCA cycle and the electron transport chain, thus contributing to reduced oxidative capacity. Overall, carp M1 but not M2 macrophages show reduced oxidative metabolism and increased glycolysis.

To date, immunometabolic reprogramming of polarized macrophages has been demonstrated primarily in mice, where polarized macrophages show opposing pathways for energy metabolism: M2 rely on oxidative phosphorylation whereas M1 are metabolically reprogrammed towards glycolysis. Our results indicate that carp M1 macrophages alter their energy metabolism in a manner similar to what has been described for murine M1 macrophages. On the other hand, carp M2 macrophages did not significantly alter their energy metabolism from control cells. Using real time measurements similar to the ones applied in the present study for carp, M1 murine macrophages were shown to reprogram their energy metabolism towards glycolysis [12-27].

At basal level, murine M1 macrophages show increased glycolysis and reduced oxidative phosphorylation. When pushed towards maximal capacity, murine M1 macrophages show a drastic decrease in maximal respiration and spare respiratory capacity. This metabolic reprogramming appears to be responsible for their inability to repolarize from M1 to M2, as they do not regain their oxidative capacity upon repolarization, whereas M2 can repolarize into M1 macrophages without problems (Van den Bossche et al., 2016). At basal level, carp M1 macrophages did not show the increased glycolysis and reduced oxidative phosphorylation observed for murine M1 macrophages. This could be because the initial reprogramming of carp LPS-stimulated macrophages towards glycolysis had already been normalized at the start of our measurements. The absence of differences at basal level could be the result of several differences in experimental circumstances between the studies on macrophages of mouse and carp, among which the exact origin of macrophages, stimuli and temperature. However, the absence of difference at basal level may also suggest that carp M1 macrophages were not terminally differentiated by LPS and could possibly still repolarize from M1 to M2, a hypothesis of interest for future studies. Overall, and similar to what has been observed for murine macrophages, carp M1 macrophages show reduced oxidative capacity when pushed to maximal respiration (Figure 5.6A and B). Although the absolute difference between polarized M1 and M2 macrophages appears smaller in carp than in mice, the 
energy metabolism of carp M1 macrophages appears similar to that of murine M1 macrophages.

In this study, we gained important insights into the metabolic pathways used by carp M1 macrophages and compared these to the metabolic pathways described for M1 polarized macrophages of mice (Figure 5.6C). Carp M1 macrophages
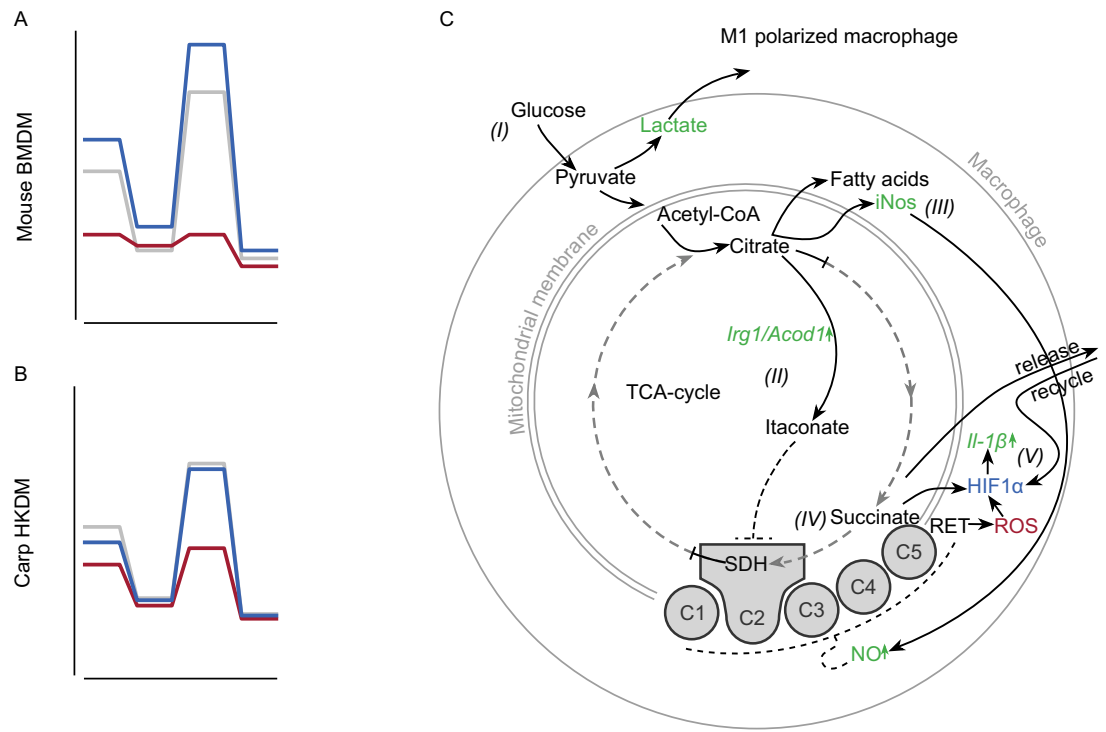

Figure 5.6 Schematic representation of metabolic reprogramming of carp M1 macrophages upon stimulation with LPS. Schematics of oxygen consumption rates (OCR) for (A) murine bone marrow derived macrophages (BMDM; based on Van den Bossche et al., 2015; Van den Bossche et al., 2016) and (B) carp head kidney-derived macrophages (HKDM; this study). Colors represent control macrophages (grey), polarized M1 macrophages (red) or M2 macrophages (blue). (C) Schematic representation of metabolic reprogramming towards glycolysis in murine macrophages, but modified for carp M1 macrophages. Pathways in black are enhanced and pathways in grey with dashed lines are decreased in murine macrophages. Text in green refers to intermediates with regulation in LPS-stimulated carp macrophages similar to regulation in mice. Text in blue refers to intermediates present in zebrafish but not yet studied in carp. Text in red refers to mechanisms present in carp M1 macrophages but not regulated similar to those in mice. Figure based on [6].

Description: (I) Increased lactate in culture supernatants suggests increased glycolysis. (II) LPSstimulated macrophages show increased irg1 expression and (III) increased inos gene expression and NO production. (IV) Reduced oxidative capacity suggests inhibition of the ETC to some degree but the mechanism needs confirmation. Succinate accumulation could not be confirmed at this point. (V) Hypoxia-inducible factor 1-alpha (Hif1 $\alpha$ ) stabilization exists under inflammatory conditions and is linked to increased $i l-1 \beta$ transcription in zebrafish $[16,17]$. Increased $i l-1 \beta$ expression has been shown in carp [14], but causation has yet to be determined. ROS production occurs in carp macrophages but is low after LPS stimulation [28]. Both succinate and ROS can theoretically stabilize Hif-1 $\alpha$, but the mechanisms need to be confirmed for carp. 
increase lactate production and shift towards glycolysis immediately after stimulation with LPS, although the exact kinetics remain to be studied. Although we could not detect differences between M1 and M2 in citrate accumulation, we did detect an upregulation of irg1 expression, which potentially leads to increased itaconate. In mice, both itaconate and NO can contribute to an inhibition of the electron transport chain. Although we can detect increased inos gene expression and increased production of NO in carp M1 macrophages, contribution of itaconate and/or NO to inhibition of the ETC in carp macrophages remains to be studied. Furthermore, although we previously reported an upregulation of il-1 $\beta$ in macrophages stimulated with LPS $[10,14]$ and although it is known that Hif1 $\alpha$ is stabilized and linked to il-1 $\beta$ expression during mycobacterium infection of zebrafish [16, 17], it remains to be confirmed if Hif1 $\alpha$ stabilization is required for il-1 $\beta$ expression in carp. Since we do not generally observe ROS production by carp macrophages in response to LPS [28] and were not able to measure intracellular succinate, it remains to be determined which of the two would contribute to the stabilization of Hif1 $\alpha$. Overall, we provide evidence of clear similarities as well as differences between polarized macrophages of mouse and carp.

Carp M2 macrophages did not show a clear increase in maximal respiration compared to controls. Moreover, differences between basal and maximal capacity appeared to be relatively small when compared to those of mice (Van den Bossche et al., 2016). Again, differences in experimental conditions between the studies on macrophages of mouse and carp, among which the exact origin of macrophages, stimuli and temperature can maybe help explain such differences. However, respiration in carp macrophages may also be regulated within more narrow boundaries than in mice: controlled use of oxygen may be particularly important in animals which breathe under water where available oxygen levels can be more often critical than in air. Studies into the effect of oxygen availability on cellular energy metabolism, in particular the metabolic reprogramming of innate immune cells, may therefore be of high interest for aquatic animals. Furthermore, oxygen availability is inversely related to temperature [29] and temperature can also directly influence mitochondrial function. For example, at lower temperatures composition of the mitochondrial membrane changes to counteract reduced membrane fluidity, which in turn changes ADP affinity of the mitochondria (reviewed by [30]. Temperature may thus play an important role in metabolic reprogramming. Carp are ectothermic fish that can be acclimatized to a large temperature range 
and a large range of oxygen pressure, which makes our model adaptable to study mitochondrial functioning and metabolic reprogramming of innate immune cells under varying environmental conditions.

Our studies confirm the general conservation of carp macrophage immune function with respect to their ability to polarize towards a pro- or anti-inflammatory profile in response to conventional M1 or M2 stimuli, and further studies could refine the extent of this conservation. Our studies also help to improve the understanding of fundamental mechanisms underlying energy metabolism and metabolic reprogramming of immune cells in teleost fish and open a field of comparative immunometabolism for exothermic aquatic vertebrates.

\section{List of abbreviations}

ADP, adenosine diphosphate; ATP, adenosine triphosphate; BMDM, bone marrowderived macrophages;cAMP, $\mathrm{N}^{6}, 2^{\prime}$-O-dibutryladenosine 3':5'-cyclic monophosphate sodium; ECAR, extracellular acidification rate; ETC, electron transport chain; FCCP, carbonyl cyanide-4 (trifluoromethoxy) phenylhydrazone; HIF1 $\alpha$, hypoxia-inducible factor-alpha; KDM, head kidney-derived macrophages; IFN- $\gamma$, interferon-gamma; IL-1 $\beta$, interleukin 1-beta; IL-4, interleukin 4; Irg1/Acod1, immune responsive gene 1/aconitate decarboxylase 1; LPS, lipopolysaccharide; NO, nitric oxide; OCR, oxygen consumption rate; OXPHOS, oxidative phosphorylation; PCS, pooled carp serum; RET, reverse electron transport; ROS, reactive oxygen species; SDH, succinate dehydrogenase; TCA cycle, tricarboxylic acid cycle.

\section{Conflict of interest}

The authors declare that the research was conducted in the absence of any commercial or financial relationships that could be construed as a potential conflict of interest.

\section{Author Contributions}

AW, JJ, VB and GW contributed to the design of the experiments. AW performed experiments and $\mathrm{AW}, \mathrm{WV}, \mathrm{MF}$ and $\mathrm{GW}$ contributed to analysis of data. GW acquired funding. AW drafted the manuscript and GW, WV, MF, JJ and VB critically reviewed the manuscript. 
Chapter 5

\section{Funding}

This research was funded by the European Commission under the 8th (H2020) Framework Program for Research and Technological Development of the European Union (PARAFISHCONTROL Grant No. 634429). JJ was funded by the NWO-WIAS Graduate Programme 2016 Grant (Wageningen Institute of Animal Sciences (WIAS) and the Dutch Organization for Scientific Research (NWO)).

\section{Acknowledgements}

Marleen Scheer is gratefully acknowledged for her assistance in obtaining head kidney leukocytes. 


\section{References}

1. Xue J, Schmidt S V, Sander J, Draffehn A, Krebs W, Quester I, De Nardo D, Gohel TD, Emde M, Schmidleithner L, Ganesan H, Nino-Castro A, Mallmann MR, Labzin L, Theis H, Kraut M, Beyer M, Latz E, Freeman TC, Ulas T, Schultze JL (2014) Transcriptome-based network analysis reveals a spectrum model of human macrophage activation. Immunity 40:274-288. doi:10.1016/j. immuni.2014.01.006.

2. Mills CD, Kincaid K, Alt JM, Heilman MJ, Hill AM (2000) M-1/M-2 Macrophages and the Th1/ Th2 Paradigm. J Immunol 164:6166-6173. 10.4049/jimmunol.164.12.6166.

3. Nathan CF, Hibbs JB (1991) Role of nitric oxide synthesis in macrophage antimicrobial activity. Curr Opin Immunol 3:65-70. doi:https:10.1016/0952-7915(91)90079-G.

4. Fang FC (2011) Antimicrobial actions of reactive oxygen species. MBio 2:e00141-11. doi:10.1128/mBio.00141-11.

5. O’Neill LAJ, Kishton RJ, Rathmell J (2016) A guide to immunometabolism for immunologists. Nat Rev Immunol 16:553-365. doi: 10.1038/nri.2016.70.

6. Van den Bossche J, O’Neill LA, Menon D (2017) Macrophage Immunometabolism: Where Are We (Going)? Trends Immunol 38:395-406. doi:10.1016/j.it.2017.03.001.

7. Tannahill GM, Curtis AM, Adamik J, Palsson-McDermott EM, McGettrick AF, Goel G, Frezza C, Bernard NJ, Kelly B, Foley NH, Zheng L, Gardet A, Tong Z, Jany SS, Corr SC, Haneklaus M, Caffrey BE, Pierce K, Walmsley S, Beasley FC, Cummins E, Nizet V, Whyte M, Taylor CT, Lin H, Masters SL, Gottlieb E, Kelly VP, Clish C, Auron PE, Xavier RJ, O’Neill LAJ (2013) Succinate is an inflammatory signal that induces IL-1 $\beta$ through HIF-1 $\alpha$. Nature 496:238-242. doi:10.1038/ nature11986.

8. Littlewood-Evans A, Sarret S, Apfel V, Loesle P, Dawson J, Zhang J, Muller A, Tigani B, Kneuer R, Patel S, Valeaux S, Gommermann N, Rubic-Schneider T, Junt T, Carballido JM (2016) GPR91 senses extracellular succinate released from inflammatory macrophages and exacerbates rheumatoid arthritis. J Exp Med 213:1655-1662. doi:10.1084/jem.20160061.

9. O’Neill LAJ, Artyomov MN (2019) Itaconate: the poster child of metabolic reprogramming in macrophage function. Nat Rev Immunol 19:273-281. doi:10.1038/s41577-019-0128-5.

10. Joerink M, Ribeiro CMS, Stet RJM, Hermsen T, Savelkoul HFJ, Wiegertjes GF (2006) Head Kidney-Derived Macrophages of Common Carp (Cyprinus carpio L.) Show Plasticity and Functional Polarization upon Differential Stimulation. J Immunol 177:61-69. doi:10.4049/ jimmunol.177.1.61.

11. Forlenza M, Fink IR, Raes G, Wiegertjes GF (2011) Heterogeneity of macrophage activation in fish. Dev Comp Immunol 35:1246-1255. doi:10.1016/j.dci.2011.03.008.

12. Wiegertjes GF, Wentzel AS, Spaink HP, Elks PM, Fink IR (2016) Polarization of immune responses in fish: The 'macrophages first' point of view. Mol Immunol 69:146-156. doi:10.1016/j. molimm.2015.09.026.

13. Saeij JPJ, Stet RJM, Groeneveld A, Verburg-van Kemenade LBM, van Muiswinkel WB, Wiegertjes GF (2000) Molecular and functional characterization of a fish inducible-type nitric oxide synthase. Immunogenetics 51:339-346. doi:10.1007/s002510050628. 
14. Piazzon MC, Savelkoul HFJ, Pietretti D, Wiegertjes GF, Forlenza M (2015) Carp Il10 Has Anti-Inflammatory Activities on Phagocytes, Promotes Proliferation of Memory T Cells, and Regulates B Cell Differentiation and Antibody Secretion. J Immunol 194:187-199. doi:10.4049/ jimmunol.1402093.

15. Arts JAJ, Tijhaar EJ, Chadzinska M, Savelkoul HFJ, Verburg-van Kemenade BML (2010) Functional analysis of carp interferon- $\gamma$ : Evolutionary conservation of classical phagocyte activation. Fish Shellfish Immunol 29:793-802. doi:10.1016/J.FSI.2010.07.010.

16. Elks PM, Brizee S, van der Vaart M, Walmsley SR, van Eeden FJ, Renshaw SA, Meijer AH (2013) Hypoxia Inducible Factor Signaling Modulates Susceptibility to Mycobacterial Infection via a Nitric Oxide Dependent Mechanism. PLoS Pathog 9:e1003789. doi:10.1371/journal. ppat.1003789.

17. Ogryzko N V, Lewis A, Wilson HL, Meijer AH, Renshaw SA, Elks PM (2019) Hif-1 $\alpha$-Induced Expression of Il-1 $\beta$ Protects against Mycobacterial Infection in Zebrafish. J Immunol 202:494502. doi:10.4049/jimmunol.1801139.

18. Hodgkinson JW, Fibke C, Belosevic M (2017) Recombinant IL-4/13A and IL-4/13B induce arginase activity and down-regulate nitric oxide response of primary goldfish (Carassius auratus L.) macrophages. Dev Comp Immunol 67:377-384. doi:10.1016/j.dci.2016.08.014

19. Irnazarow I (1995) Genetic variability of Polish and Hungarian carp lines. Aquaculture 129:215. doi:10.1016/0044-8486(95)91961-t.

20. Zapata A, Amemiya CT (2000) Phylogeny of Lower Vertebrates and Their Immunological Structures BT. In: Du Pasquier L, Litman GW (eds) Origin and Evolution of the Vertebrate Immune System. Springer Berlin Heidelberg, Berlin, Heidelberg, pp 67-107

21. Saeij JPJ, Van Muiswinkel WB, Groeneveld A, Wiegertjes GF (2002) Immune modulation by fish kinetoplastid parasites: a role for nitric oxide. Parasitology 124:77-86. doi:10.1017/ S0031182001008915.

22. Kolder ICRM, van der Plas-Duivesteijn SJ, Tan G, Wiegertjes GF, Forlenza M, Guler AT, Travin DY, Nakao M, Moritomo T, Irnazarow I, den Dunnen JT, Anvar SY, Jansen HJ, Dirks RP, Palmblad M, Lenhard B, Henkel C V, Spaink HP (2016) A full-body transcriptome and proteome resource for the European common carp. BMC Genomics 17:701. doi:10.1186/s12864-016-3038-y.

23. Petit J, Bailey EC, Wheeler RT, de Oliveira CAF, Forlenza M, Wiegertjes GF (2019) Studies Into $\beta$-Glucan Recognition in Fish Suggests a Key Role for the C-Type Lectin Pathway. Front Immunol 10:280. doi:10.3389/fimmu.2019.00280

24. Love MI, Huber W, Anders S (2014) Moderated estimation of fold change and dispersion for RNA-seq data with DESeq2. Genome Biol 15:550. doi:10.1186/s13059-014-0550-8.

25. Van den Bossche J, Baardman J, de Winther MPJ (2015) Metabolic Characterization of Polarized M1 and M2 Bone Marrow-derived Macrophages Using Real-time Extracellular Flux Analysis. J Vis Exp 53424. doi:10.3791/53424.

26. Van den Bossche J, Baardman J, Otto NA, van der Velden S, Neele AE, van den Berg SM, LuqueMartin R, Chen H-J, Boshuizen MCS, Ahmed M, Hoeksema MA, de Vos AF, de Winther MPJ (2016) Mitochondrial Dysfunction Prevents Repolarization of Inflammatory Macrophages. Cell Rep 17:684-696. doi:10.1016/j.celrep.2016.09.008. 
27. Vijayan V, Pradhan P, Braud L, Fuchs HR, Gueler F, Motterlini R, Foresti R, Immenschuh S (2019) Human and murine macrophages exhibit differential metabolic responses to lipopolysaccharide - A divergent role for glycolysis. Redox Biol 22:101147. doi:10.1016/j.redox.2019.101147.

28. Pijanowski L, Scheer M, Verburg-van Kemenade BML, Chadzinska M (2015) Production of inflammatory mediators and extracellular traps by carp macrophages and neutrophils in response to lipopolysaccharide and/or interferon- $\gamma 2$. Fish Shellish Immunol 42:473-482. doi:10.1016/j.fsi.2014.11.019.

29. Wetzel RG (2001) Oxygen. In: WETZEL RGBT (ed) Limnology, Third edit. Academic Press, San Diego, pp 151-168.

30. O’Brien KM (2011) Mitochondrial biogenesis in cold-bodied fishes. J Exp Biol 214:275 LP - 285. doi:10.1242/jeb.046854. 

General discussion

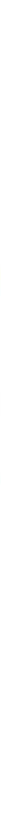




\section{A short recap of outcomes}

To date, mammalian macrophages are recognized to polarize into a whole spectrum of functional phenotypes with the polarized extremes (M1 and M2) showing largely opposite functions. In this thesis, we investigated carp macrophages (Cyprinus carpio) polarized to M1 and M2 phenotypes. We started by reviewing both, the indications for the existence of polarized macrophages in fish and the 'macrophages first' hypothesis in chapter 2 . We also systematically reviewed the evidence in fish for the existence and conservation of conventional polarizing stimuli of mammalian macrophages. Subsequently, we performed extensive transcriptomic analyses to determine candidate markers for M1 and M2 macrophages after polarization with well-characterized stimuli such as LPS (M1) and cAMP (M2) in chapter 3. There, we showed that M1 and M2 carp macrophages are not only functionally different, but also show distinct transcriptional profiles, which appear functionally similar to those of polarized macrophages described for humans and mice. We showed that combination of the T helper 1 cytokine Ifn- $\gamma$ with LPS induces transcriptional profiles similar to LPS, but with fold changes in gene expression that were higher than with LPS alone. This suggests that also in carp, adaptive immune components can enhance already polarized innate immune cells to become even more potent. To increase our understanding of the downstream effects of M1 polarization on other inflammatory innate immune cells, we studied the effect of granulocyte colonystimulating factor (Csf3) on carp neutrophils in chapter 4. We discovered that carp Csf3 is primarily expressed by M1 macrophages, that the two Csf3 paralogs that we studied increase proliferation of hematopoietic stem cells and are chemotactic towards neutrophils. Csf3b specifically increased differentiation of precursor cells towards granulocytes, while the combination of Csf3a and Csf3b induced a mixed macrophage/granulocyte phenotype. Moreover, both Csf3a and Csf3b enhanced the respiratory burst capacity of neutrophils. This indicated that M1 macrophage polarization potentiates the proliferation, activation and inflammatory function of neutrophils. The high degree of functional conservation of M1 and M2 macrophages led us to hypothesize a similarly high degree of conservation of associated metabolic profiles. Therefore, we studied the energy metabolism of M1 and M2 macrophages in real-time in chapter 5 and discovered that carp M1 macrophages show signs of metabolic reprogramming towards glycolysis similar to mammalian M1 macrophages. 


\section{Macrophages play the first violin}

Innate immunity predates the development of adaptive immunity, which only emerged in vertebrate animals, and innate immune responses are of crucial importance to host survival [1-4]. Effective innate immune responses are particularly important for invertebrate hosts without adaptive immunity but also for cold-blooded animals such as teleost fish, for which adaptive immune responses may be delayed or even impaired at lower temperatures [1, 5]. Even if these invertebrates or teleost fish rely primarily on innate immunity, they can still effectively build immune responses to survive infections of different nature including viral, bacterial, helminthic or fungal [6]. These observations gave rise to the idea that polarized states of innate immunity are likely evolutionarily older than polarized states of adaptive immunity and raise the question whether polarized innate responses occur earlier in infection, before fully developed adaptive immune responses are in place. In this scenario, polarized innate immune cells would activate and steer adaptive immune components, which in turn would enhance (already polarized) innate immune cells to become even more potent. This concept became known as the 'macrophages first' theory ([7], chapter 2), because macrophages are considered prime orchestrators of innate immune responses, especially in lower vertebrates. In this thesis, we set out to study polarized innate immune responses using macrophages as primary cell type.

\section{Growth factors, functionally conserved or not?}

Mammalian macrophages can be polarized in vitro when grown in the presence of the colony-stimulating growth factors GM-CSF (M1) or M-CSF (M2), or in the presence of the T helper 1 cytokine Ifn- $\gamma$ (M1) or T helper 2 cytokines IL- 4 or IL13 (M2). The effect of the cytokine Ifn- $\gamma$ on carp macrophages and associated identification of M1 markers has been discussed extensively in chapter 3. To study potential M2 markers it was not evident to copy the experimental approach used for mammalian macrophages, because a clear and functional conservation of M-CSF, IL-4 and IL-13 had not been proven for fish and a clear GM-CSF paralog had not been identified. Nevertheless, future studies on the potential functional conservation of these growth factors and polarizing cytokines in teleost fish and their effect on fish macrophages remain of great interest. 
In mammals, granulocyte macrophage colony stimulating factor (GM-CSF) is a hematopoietic growth factor also known as CSF2 and is part of the Il-5 family together with IL-3 and IL-5. Members of this family exert effects on proliferation, differentiation, effector functions and trafficking of many leukocytes (recently reviewed by [8]) and CSF2 in particular is considered an inducer of M1 macrophages in humans and mice [9]. This particular family of genes appears poorly conserved throughout evolution and although candidate genes have been discovered based on synteny in elephant shark and several teleost fish species [10], no studies have confirmed functional conservation of these Il-5 family members, at least not with respect to M1 polarization of fish macrophages. As such, whether these Il-5 family members exert any of the functions associated with mammalian CSF2, IL-3 or IL-5 has remained undetermined. In zebrafish (danio rerio), there is one candidate il-5 family member identified based on its location next to the il-4 gene [10] (Figure 6.1). In carp, an initial genome BLAST search using the zebrafish il-5 family member revealed the presence of two paralogs, of which at least one paralog appears located to il-4/13b. It would be of great interest to study the modulatory effects of the two 'Il-5 family members' on carp macrophages to define their function as Csf2 (or not) and if so, use Csf2 (Gm-csf) to mediate macrophage polarization to M1 for in vitro studies on fish macrophages.

Macrophage colony stimulating factor (M-CSF) is a hematopoietic growth factor also known as CSF1. In mammals, it is primarily used to induce M2 macrophages and is known to stimulate (in vitro) proliferation, differentiation and activation of monocytes and macrophages [9]. Initially, studies in fish indicated Csf1 could exert primarily inflammatory effects, suggestive of M1 rather than M2 polarization. More recent studies combining recombinant Csf1 with recombinant Il-4 indicate a synergistic proliferative effect on fish macrophages, further spiking our interest in the functional phenotype of such Csf-1-stimulated cells [11]. If Csf1 and Il-4 would also synergize to induce anti-inflammatory functions, this would indicate that Csf1 (M-csf) could be used to mediate macrophage polarization to M2 for in vitro studies on fish macrophages.

\section{Thelper 2 cytokines in fish: interleukin 4 and/or 13}

Interleukin-4 and IL-13 are cytokines with comparable function in mammals that appear the be the result of a tandem duplication of an ancestral gene also present in fish (reviewed by [12]). In fish, this ancestral gene is referred to as $I l-4 / 13$ because 
the sequence shows no greater resemblance to either IL-4 or IL-13. Primarily based on synteny with neighboring genes, il4/13 sequences have now been described for multiple fish species [10,12-15]. The ancestral gene has not only been tandemly duplicated in mammals to form IL- 4 and IL-13 but has also been duplicated by whole genome duplication (WGD) and/or other lineage-specific tandem duplication events in fish. The presence of two copies is common in most teleost fish species owing to the teleost-specific $3^{\text {rd }}$ round of whole genome duplication (3R-WGD) [16]. It is not uncommon to find sub-functionalization of multiple paralogs [17-22] which can be the results of WGD or tandem duplication events and can result in functional differences between paralogs in both function and target.

Sequence similarity of the il4/13 genes in fish to either Il4 or Il13 genes in mammals is low $[12,13]$, making it difficult to predict function based on sequence information only. Recombinant proteins of teleost Il-4/13 have been shown to induce arginase activity in vitro, in macrophages of goldfish (Carassius auratus)[23] and in macrophages of grass carp (Ctenopharyngodon idella) [11], hinting at a conservation of M2-inducing properties of Il-4/13 in fish. Based on the close sequence similarity of an il4/13b described for Asian common carp (AB690830 in Figure 6.2) [24] and a il4/13b1 sequence of European common carp we identified, we used the former Il-4/13b recombinant protein for our studies on carp macrophages (chapter 3). Despite evident bioactivity of the recombinant protein as shown by its proliferative effects on carp B-cells [24] and other modulatory effects on carp leukocytes (our data), Il-4/13b1 did not induce arginase activity or transcriptional changes in carp macrophages. Later, this particular Il-4/13b1 paralog was suggested by others to be linked more to $\mathrm{T}$ cell rather than innate immune activation, at least in sea bass (Dicentrarchus labrax) [15]. This could suggest that the Il-4/13b1 paralog we studied may have sub-functionalized towards activating B or T cells, rather than towards activating macrophages. Similarly, there may be sub-functionalization of Il-4/13 sensitive receptor complexes, a possibility not unlikely but largely unstudied so far.

Teleost fish species generally express at least two paralogs of $i l 4 / 13$ as a result of 3R-WGD, while common carp can be expected to have up to four gene copies due to an additional whole genome duplication in some cyprinids (4R-WGD) [20, 25-28]. If indeed four il4/13 paralogs would be present in the carp genome, it cannot be excluded that one or more of the other (non-investigated) paralogs could be capable of inducing an M2-like polarization. We therefore investigated the presence of more 
paralogs in the draft genome of European common carp [29] using a combination of synteny and blast analysis using published il4/13 sequences of carp and related cyprinid fish species including zebrafish and goldfish [13, 23]. In zebrafish, il13 (better known as il4/13a) is located on chromosome 9 next to Rad50, a syntenic gene to human and mouse $I L-13$ (Figure 6.1). Zebrafish il4 (better known as il4/13b) is located on chromosome 11 next to kif3a, a syntenic gene to human and mouse $I L-4$. We found convincing evidence for the presence of multiple sequences for these molecules in the carp genome (Figure 6.1), where (partial) sequences for $i l 4 / 13 a$ appear present on the same contigs that also contain Il4/13a2 and Il4/13a3. The finding of a tandem duplication for $i l-4 / 13 a$ is not completely unexpected because this was also reported in sea bass [15]. However, it is important to realize that the carp il-4/13 sequences we identified are preliminary and need to be completed and confirmed as their exact organization awaits an improved version of the carp genome.

Phylogenetic comparison of protein sequences from translated genes show that carp Il-4/13a and Il-4/13b cluster together with their cyprinid relatives, including zebrafish, grass carp and goldfish, in both neighbor-joining (Figure 6.2) and

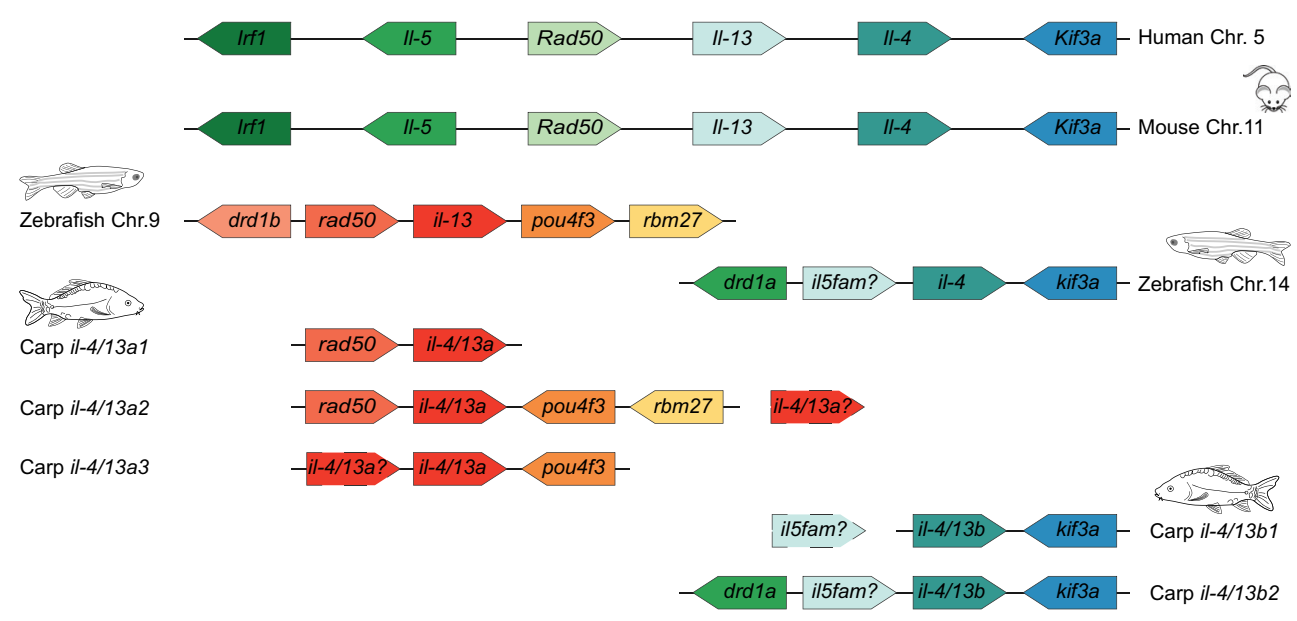

Figure 6.1 Synteny analysis of il-4/13 paralogs from carp shows a high degree of conservation compared to zebrafish and mammals. Equal colors indicate conserved genes and chromosomal representations are not drawn to scale. Genomic organization of human, murine and zebrafish chromosomes were based on ensemble databases and [13]. Partial sequences for an additional il-4/13a gene appear present on the scaffolds of $i l-4 / 13 a 2$ and $i l-4 / 13 a 3$ and are indicated with a question mark (?), but these remain to be confirmed in an improved version of the carp genome. 


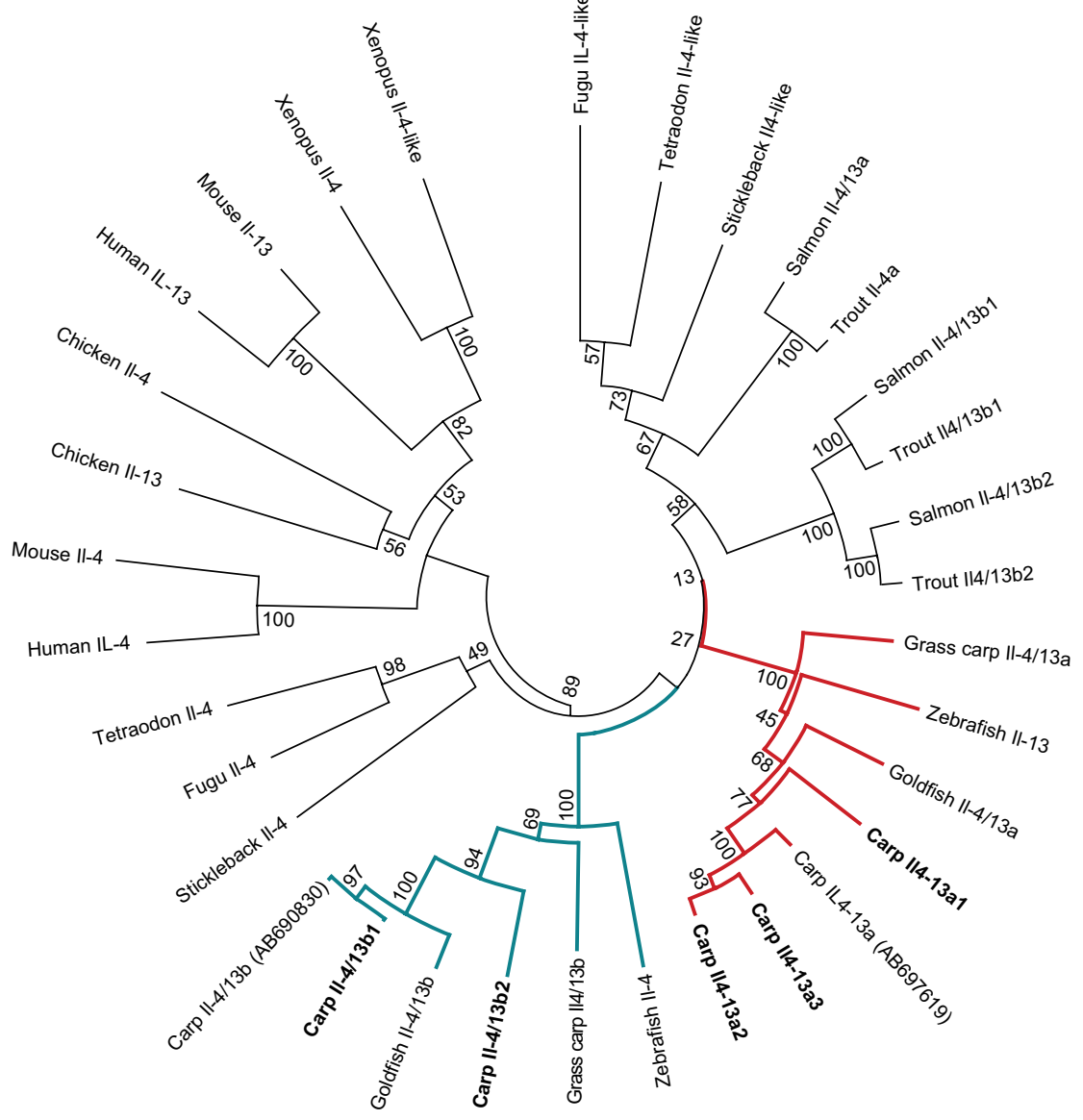

Figure 6.2 Phylogenetic analysis of carp Il-4/13 protein sequences by the neighbour-joining method. Red lined indicate the cyprinid Il-4/13a cluster and blue lines indicate the cyprinid Il-4/13b cluster. Bold sequences indicate carp Il-4/13 consensus sequences derived from genomic analysis, which were completed using PCR analysis. Other carp Il-4/13 sequences represent previously published Il4/13a and Il-4/13b sequences from Asian common carp (Cyprinus carpio) [24, 30]. Tetraodon (Tetraodon nigrovirides), fugu (Fugu rubripes) and stickleback (Gasterosteus aculeatus) Il-4/13 sequences were derived from [13], Genbank accession numbers for other sequences are the following: human (Homo sapiens) IL-4, NP_000580.1; human IL-13 AAC03535.1; mouse (Mus musculus) Il-4, NP_067258.1; mouse Il-13, NP_032381.1; chicken (Gallus gallus) Il-4, NP_001007080.1; chicken Il-13, NP_001007080.1; xenopus (Xenopus tropicalis) Il-4, NP_001107279.1; xenopus Il-4 like, KAE8614542.1; zebrafish Il-4, NP_001164211.1; zebrafish (Danio rerio) Il-13, NP_001186834; trout (Oncorhynchus mykiss) Il-4/13a, NP_001233270.1; trout Il4/13b1, CDK69043.1; trout Il4/13b2, CDK69044.1; salmon (Salmo salar) Il4/13a, NP_001191824.1; salmon Il4/13b1, CDK69045.1; salmon Il-4/13b2, CDK69046.1; goldfish Il-4/13a, AOH73291.1; goldfish (Carassius auratus) Il-4/13b, AOH73292.1; grass carp (Ctenopharyngodon idella) Il-4/13a, QGD14190.1; grass carp Il4/13b, QGD14191.1. Numbers indicate the number of times the associated taxa clustered together in a bootstrapping test (100 replicates). 
maximum likelihood trees. Where Il4/13b sequences align well, Il-4/13a sequences show high sequence diversity (63-86 \% amino-acid identity) with Il-4/13a from Asian common carp (AB697619) [30]. This could help explain why recombinant Il-4/13a from Asian common carp did not exert effects on macrophages from European common carp in our preliminary study (data not shown). In conclusion, it seems obvious that the next approach should be to produce European common carp-specific recombinant proteins, preferably for all paralogs but at least based on il4/13b2 and il4/13a1-3 sequences to further study the effects of Il-4/13 on macrophage polarization in carp.

\section{Inflammatory macrophages: M1 only, or part of a spectrum?}

We scrutinized the robustness of the inflammatory phenotype and marker profile we identified for M1 macrophages polarized by LPS alone and by LPS + Ifn- $\gamma$ (chapter 3 ). For this, we cross-referenced the genes that were highly upregulated by these stimuli against genes highly upregulated in macrophages stimulated by zymosan. Zymosan consists of yeast-derived particles that induce inflammatory responses in macrophages via the NF-kb pathway [31] and appear to signal through a C-type lectin pathway in carp [32]. Thus, we expected zymosan to induce an inflammatory phenotype which could be comparable but not identical to M1 macrophages, and at least different from M2 macrophages that show an anti-inflammatory phenotype.

Indeed, the transcriptional phenotype of zymosan-stimulated carp macrophages proved inflammatory because most of the highly (log2 fold change > 1.5) upregulated genes (37) fell within the (much larger) inflammatory repertoire of LPS (+ Ifn- $\gamma$ ) -induced M1 macrophages (Figure 6.3A). Studying the 'common' inflammatory phenotype, we noticed that of the overlapping subset of 38 genes, 26 could be highlighted as common to inflammatory macrophages (Figure 6.3A). Of these, six genes were previously proposed as candidate M1 markers based on their expression profiles in LPS-stimulated macrophages (Figure 6.3B). Additionally, there were 11 genes upregulated in all activated macrophage sub-types (including M2), 2 of which at least fourfold (log2 fold change $>2$ ) higher in zymosan-stimulated than in M2 macrophages (Figure 6.3B). In conclusion, we could identify eight genes that appear good 'common' markers for inflammatory macrophages (Figure 6.3B). 
The overlap in upregulated genes between LPS- and zymosan-stimulated macrophages indicates that also yeast-derived zymosan could be classified as inflammatory. Therefore, the phenotype of zymosan-stimulated macrophages could be placed towards M1, at the inflammatory side of the macrophage spectrum. There was a subset of eight markers, consistent across all inflammatory stimuli, that appear good 'common' markers for inflammatory macrophages. These include the canonical M1 marker inos (nos2b), but also olfm 4, which is involved in myeloid differentiation and neutrophil inflammation, and two paralogs of the Il1 receptor 2 . Of particular interest is il12p35, previously (chapter 3) highlighted as an interesting marker for M1 macrophages because i) two paralogs were increased in M1 and ii) it was the only candidate (almost) not expressed in control and M2 macrophages (see also Figure 6.4). In combination with macrophage-specific (mpeg) labelling il12p35 could indeed be an interesting marker to label M1 macrophages in zebrafish models. At the same time, the consistent upregulation of il12p35 across all inflammatory stimuli investigated here also indicates $i l 12 p 35$ as a useful and ubiquitous marker for inflammatory macrophages.
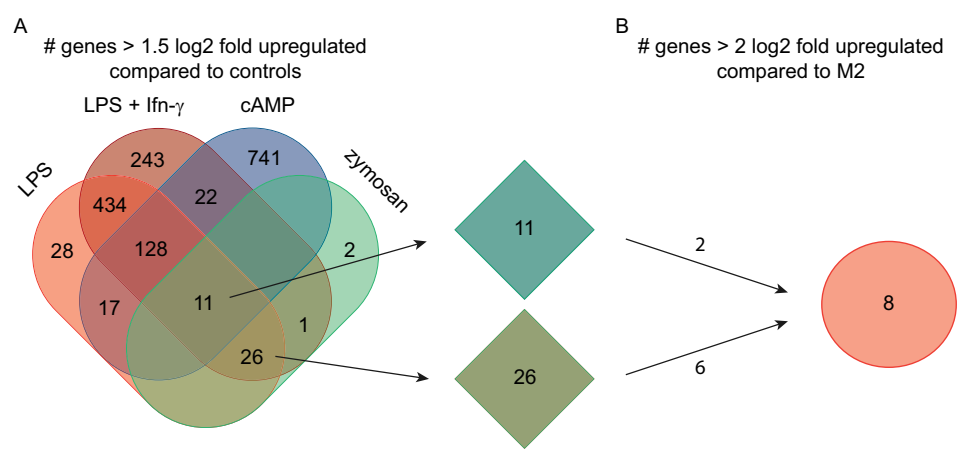

\begin{tabular}{ll} 
cypCar__ & gene \\
\hline $00002502-$ & mecr \\
00002503 & \\
00024970 & $i l 1 r 2$ \\
00015036 & slc2a6 \\
00024699 & il12p35 \\
00033150 & il1r2 \\
00004424 & nos2b \\
00026281 & irg1 \\
00074183 & olfm4 \\
\hline
\end{tabular}

Figure 6.3 Identification of 'common' marker genes for inflammatory macrophages. Only predicted genes that are significantly $\left(p_{\text {adiusted }}=0.05\right.$, analyzed by DESeq2 [33]) and at least $1.5 \log 2$ fold upregulated compared to unstimulated controls are shown for all groups. A) Number of predicted genes upregulated in macrophages polarized towards M1 using LPS+ Ifn- $\gamma$ (dark red) or LPS alone (bright red) or polarized towards M2 (blue) for $6 \mathrm{~h}$, and in inflammatory macrophages stimulated with Zymosan for $6 \mathrm{~h}$ (green). B) Eight potential M1 markers are also valid for Zymosan-stimulated macrophages. These markers are at least $1.5 \log 2$ fold upregulated in all inflammatory groups and not upregulated in M2 macrophages at all $(p<0.05$ or log2 fold change $<1.5$ ) or at least $2 \log 2$ fold lower in M2 macrophages (log2 fold change inflammatory macrophages-log2 fold change M2 > 2). In the case of mecr, two predicted genes encoded by two different cypCar numbers likely represent one actual gene. CypCar numbers represent predicted genes and are accompanied by the abbreviated gene names [29]. 
The use of Il-12p35 as a macrophage marker is complicated by the dimerization of different subunits of the Il-12 family to form multiple cytokines. As such, the p35 subunit might not only function as part of pro-inflammatory Il-12, but may also be expressed as part of anti-inflammatory IL-35 [34] (Figure 6.4). Although IL-35 production has been primarily attributed to T-regulatory cells and thus should not be highly expressed in macrophages, we would still need to confirm whether $\mathrm{p} 35$ expression is solely related to Il-12 production in macrophages. In fact, because of the 3R-WGD in carp one can expect the presence of multiple paralogs of both p35 and p40 subunits. Two distinct p35 subunits and three distinct p40 ( $a, b$ and c) subunits have already been described for multiple teleost fish species, including fugu, tetraodon, stickleback and zebrafish [35]. Preliminary analysis of the carp genome and transcriptome indicates the presence of four p35 paralogs and six p40 paralogs ( $2 \times$ p40a, $2 \times$ p40b and $2 \times$ p40c). Moreover, we identified two ebi3 paralogs (of which only one has been predicted) and two p19 paralogs, all of which are indicated with corresponding cypCar numbers and regulation in M1 and M2 (Figure 6.4). Two closely related p35 paralogs are highly upregulated in M1 macrophages and in concert with multiple p40 paralogs as indicated by the arrows. Although gene expression is sometimes not one to one comparable with protein production and data on the regulation of one ebi3 paralog is missing, there appears to be no expression of ebi3, which may suggest an absence of Il-35 in our macrophage cell culture system. Taken together and certainly in culture systems such as ours, a strong argument for the use of Il-12p35 as an inflammatory marker for macrophages remains.

\section{At the other side of the spectrum: anti-inflammatory M2 macrophages and close colleagues}

On the M2 side of the mammalian macrophage spectrum, the number of characterized subsets expanded over time to include those stimulated with IL-4 or IL-13 (M2a) and also macrophages stimulated with immune complexes or apoptotic cells (M2b) or signals of damage and/or the anti-inflammatory cytokine IL-10 (M2b-c). Even though identification of clear and unambiguous M2 stimuli for fish macrophages have remained challenging, one could argue that macrophages with anti-inflammatory phenotypes different from the typical cAMP-induced M2(a) phenotype should also exist in fish. In mammals the group of regulatory macrophages (M2b-M2c) is primarily characterized by their production of the anti-inflammatory cytokines IL-10 and TGF- $\beta$ [36]. To what degree M2 subsets 
IL-35

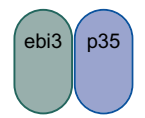

IL-12

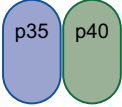

IL-23

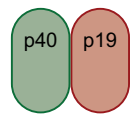

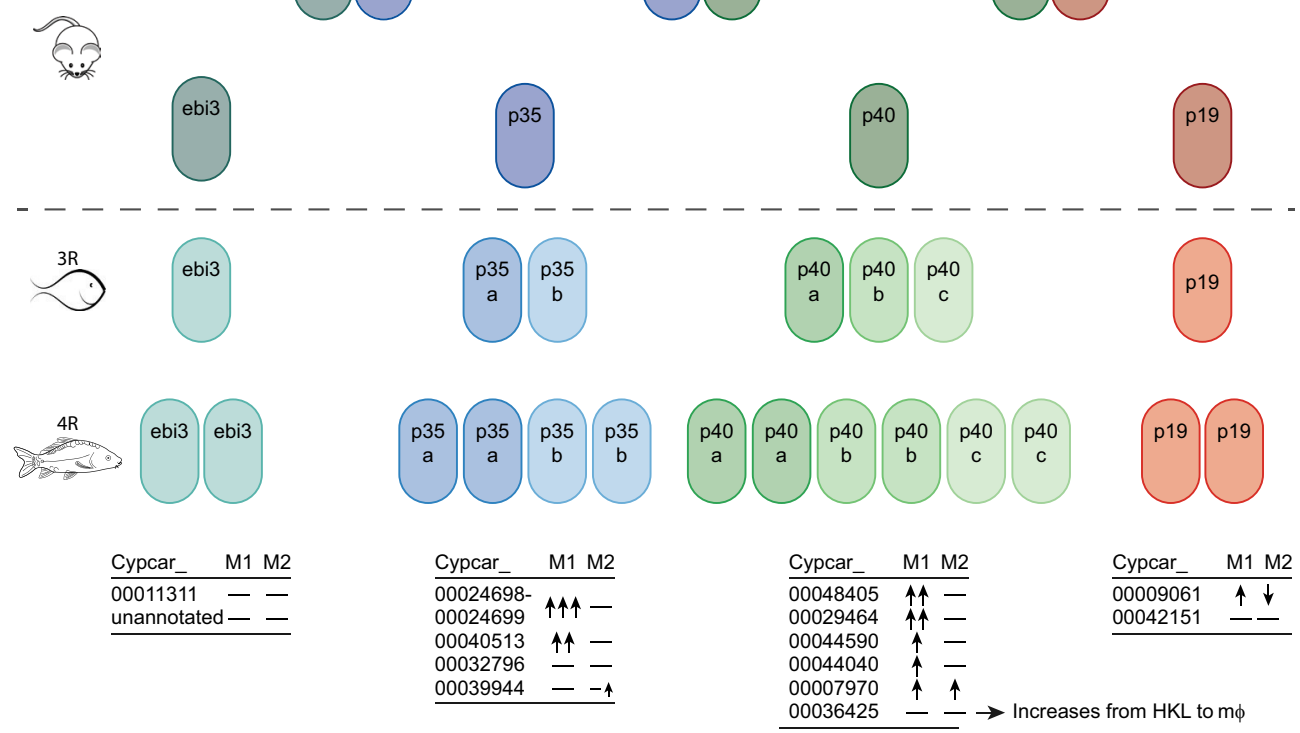

Figure 6.4 Subunits of Il-12 are similarly organized in mammals and fish and dimerize to form Il-12, Il-35 and Il-23. Schematic overview of Il-12 subunits p35 (blue) and p40 (green) and associated subunits ebi3 (teal) and p19 (red) in mammals (top), 3R-WGD teleost fish and 4R-WGD carp (bottom). Arrows indicate the increase in expression of predicted protein sequences (cypCars) encoding the different paralogs upon polarization towards M1 and M2 macrophages. Dashes indicate no significant regulation of the genes upon polarization towards M1 and M2 macrophages and the combination of an arrow and dash indicates significant but small increase and low absolute expression (cypCar_00039944).

of fish macrophages would be conserved has remained largely unknown. To elicit anti-inflammatory or regulatory activities, macrophages can be stimulated with Il-10 itself but also by immune complexes, glucocorticoids, apoptotic cells and prostaglandins [36, 37]. In line with its regulatory activity, carp Il-10 is able to downregulate LPS-induced NO production and pro-inflammatory cytokine production in carp macrophages [38, 39], although without upregulating il10. Likewise, the glucocorticoid cortisol is able to reduce pro-inflammatory responses in head-kidney macrophages/monocytes [40], although without accompanying upregulation of $i l 10$ or $\arg 2$. These data suggest that anti-inflammatory phenotypes of carp macrophages could be broader than the cAMP-induced M2(a) phenotype we described in chapter 3. 
In mammals multiple M2 associated stimuli, including IL-4, exert anti-inflammatory effects through increased intracellular cyclic adenosine monophosphate (cAMP) [41]. This second messenger causes activation of multiple downstream signals including PKA and CREB, which inhibits NF-kB transcription and induces an antiinflammatory phenotype in mammalian macrophages [41, 42] Several mediators associated with tissue damage and inflammation such as prostaglandins [41, 43] and adenosines [44] have been reported to exert their anti-inflammatory effects at least in part through accumulation of intracellular cAMP. This puts increased intracellular levels of cAMP central to activation of M2 macrophages. Indeed, we have confirmed a conserved cAMP-induced anti-inflammatory phenotype in M2 macrophages in carp (chapter 3).

We hypothesized an anti-inflammatory phenotype could be induced by cAMP agonists such as prostaglandins. Prostaglandin E2 is a lipid mediator which is produced by many cell-types and plays a complex role in inflammation. In mammals, the production of its precursor from arachidonic acid is under the control of PTGS1 (COX1) or PTGS2 (COX2) which are also responsible for production of other inflammatory mediators [45]. Final conversion to PGE2 is mediated by PGE synthase (PGES). Particularly PTGS2 is induced in early inflammation and PGE2 has been associated with important pro-inflammatory functions [46] but can also increase intracellular levels of cAMP which can negatively regulate NF-kB mediated inflammatory functions and induce an anti-inflammatory phenotype in macrophages. For example, it induces expression of il10, arg1 and $\operatorname{mrc} 1$ in murine macrophages [47]. Macrophages themselves are also considered an important source of PGE2 in mammals and production of PGE2 is induced by LPS, and associated with a negative feedback mechanism [48].

Several studies have been performed to determine the role of prostaglandin E2 or its stable synthetic analog (16,16-dimethyl-PGE2) in fish although studies in macrophages are mostly performed in trout (Oncorhynchus mykiss) and seabream (Sparus aurata). In trout, PGE2 increased phagocytosis of yeast particles [49] and downregulated the respiratory burst of macrophages [50], which indicates antiinflammatory effects of PGE2. In seabream PGE2 induced expression of il10, arginase ( $\arg )$ and the canonical M2 marker mannose receptor type $c 1$ ( $m r c 1)$ and inhibited il6 and il1 $\beta$ expression induced by bacterial DNA [51]. In our transcriptional datasets of M1 and M2 polarized carp macrophages, we find increased expression 
of PTGS2 (ptgs2a) in M1 macrophages (> 50-fold) and M2 macrophages (> 7-fold). Furthermore, we find increased ptges expression (10-fold) in M1 macrophages, indicating the early induction of prostaglandin E2. Based on these data we hypothesized 16,16-dimethyl-PGE2 (referred to as PGE2) would downregulate inflammatory responses and possibly induce anti-inflammatory phenotype in carp macrophages.

We explored a PGE2-induced M2-like phenotype in carp macrophages while simultaneously aiming to confirm some of the M2 candidate marker genes previously determined in cAMP stimulated M2 macrophages. Indeed, PGE2 downregulated il12p35 and to a degree also il $1 b$ expression induced by LPS, which indicated a negative regulation of inflammatory responses (Figure 6.5A). These results indicated that PGE2 downregulates, in part, canonical M1 markers in carp macrophages and may instead induce an M2-like phenotype. We subsequently studied arginase activity and expression of several M2 candidate marker genes. Of the 11 candidate M2 markers tested, eight were increased in PGE2-stimulated macrophages (Figure 6.5B and $\mathrm{C}$ ), the other three markers (angptl4, ramp2 and cyr61l) showed inconsistent results (data not shown). Although cumulative NO production was not altered by PGE2 and arginase activity was not increased by PGE2 (Figure 6.5F), the preliminary gene expression results point towards the possibility that PGE2 induces an M2-like phenotype in carp macrophages. As neither one of the primary mediators of Il-10 signaling socs $3 b$ nor il10b itself was upregulated by PGE2, PGE2 appears to induce an M2-like phenotype in carp macrophages without upregulating Il-10.

Il-10-stimulated profiles of carp macrophages were investigated. Our data indicate a downregulation of pro-inflammatory responses of not only NO and production of inflammatory cytokines as reported previously [38], but we also see metabolic changes (Figure 6.6A). Preliminary results from real-time metabolic experiments (see also chapter 6) indicate an Il-10-induced increase in oxygen consumption, particularly at basal level and for ATP production, while maximum respiration remains similar to control and M2 macrophages. Preliminary data on the direct effect of IL-10 alone indicate only a modest upregulation of arginase activity (Figure 6.6B) and modest upregulation of some of the proposed M2 markers (chapter 3), including tcima, adam28 and tinagl1b. More interestingly, one of the more classical M2a markers tgm $2 b$ is not upregulated but rather downregulated 
by Il-10 (Figure 6.6C) and co-stimulation experiments with PGE2 indicated Il-10 also downregulated PGE2 induced increases of more classical M2a markers $\operatorname{tgm} 2 b$ and $m r c 1$ (data not shown). Finally, also il10b expression in macrophages is downregulated by Il-10, as we previously observed, indicating a possible negative feedback loop induced by Il-10 itself (Figure 6.6C).
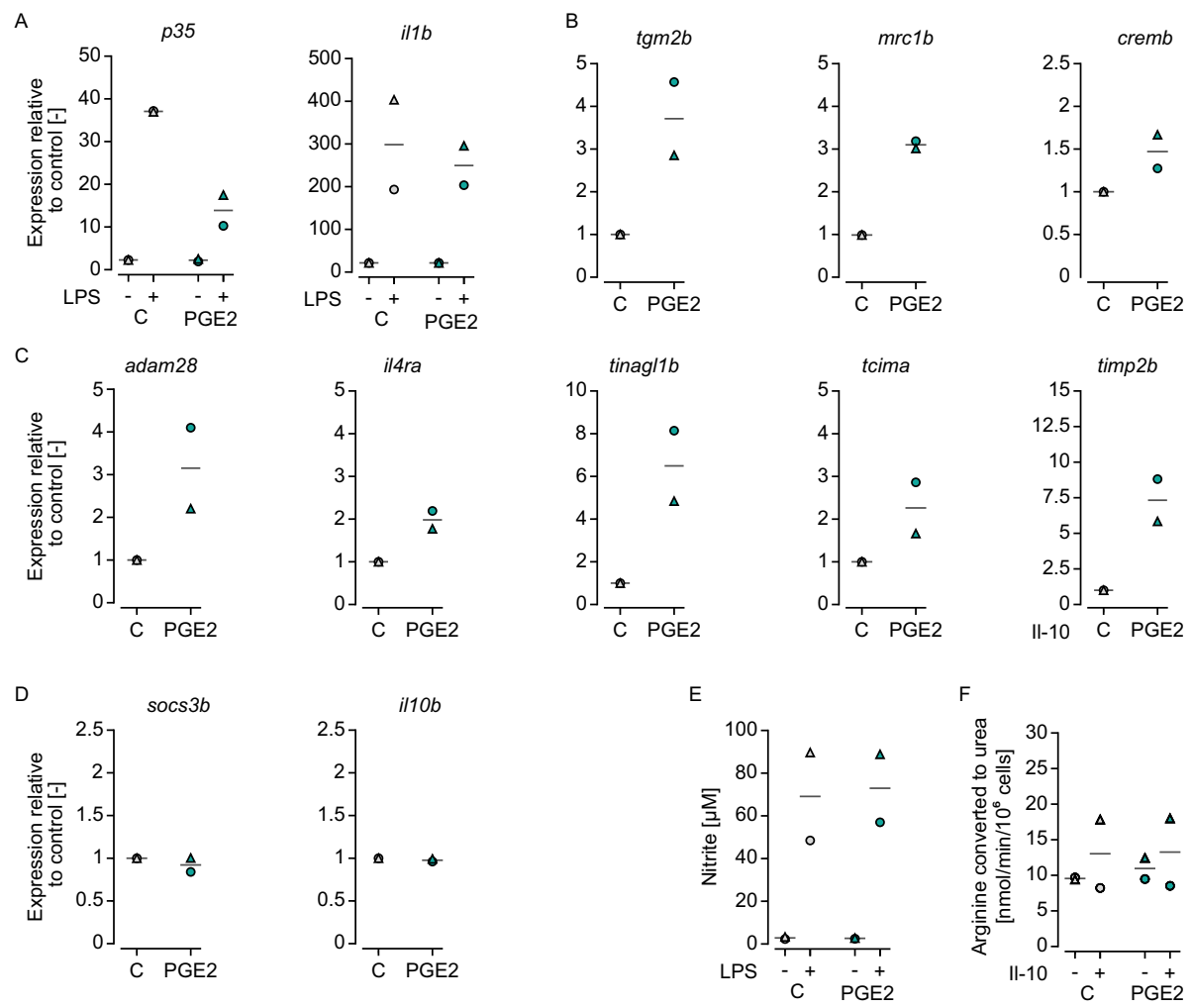

Figure 6.5 Prostaglandin E2 counteracts LPS-induced inflammation in carp macrophages and induces early upregulation of M2 marker genes in carp macrophages. Carp macrophages were stimulated with $10 \mu \mathrm{M}$ of the stable analog 16,16-dimethyl-prostaglandin E2 (PGE2, green) in the presence or absence of $10 \mu \mathrm{g} / \mathrm{ml}$ lipopolysaccharide (LPS, A, C). The PGE2 vehicle (0,0095\% ethanol) was used as control (C, grey). A) Real-time gene expression after $6 \mathrm{~h}$ of pro-inflammatory genes $p 35$ and ill $b$ using common primers. B, C) Real-time gene expression after $6 \mathrm{~h}$ of proposed M2 markers identified in chapter 3 (tgm $2 b$, mrc1b, cremb, adam 28, il4ra, tinagl1b, tcima and timp $2 b$,) using paralog specific primers (except tcima which discriminated tcima paralogs from tcimb). D) Real-time gene expression after $6 \mathrm{~h}$ of $s o c 3 b$ and $i l 10 b$. Gene expression was normalized to the $s 11$ protein of the $40 s$ subunit as a reference gene and shown as the fold change relative to the vehicle controls. E) Nitric oxide production of macrophages measured as nitrite concentration in culture supernatants after $24 \mathrm{~h}$. F) Arginase activity of stimulated macrophages after $24 \mathrm{~h}$, measured in cell lysates as conversion of L-arginine to urea by arginase in $\mathrm{nmol} /$ $\min / 10^{6}$ cells. Data are the mean and $n=2$ individual fish (symbols). 
Whether regulatory macrophages with a more characteristic phenotype (e.g. high expression of $i l 10$ ) would be induced by Il-10 over a longer period of stimulation, or by any of the other anti-inflammatory stimuli mentioned above as well as by a combination of anti-inflammatory signals, remains to be determined. Alternatively, co-activation with TLR ligands or other inflammatory triggers may be required to enhance the regulatory mechanisms. Moreover, a full transcriptional phenotype of Il-10-stimulated carp macrophages would help to determine whether regulatory phenotypes are induced by Il-10 alone or only in combination with inflammatory stimuli and would help completing the picture of the most established macrophage phenotypes in a lower vertebrate. Yet, our preliminary data suggest that Il-10 may induce a macrophage (M2c-like?) phenotype different from the more 'traditional' cAMP-induced M2(a) phenotype.

A

Basal respiration

ATP-linked respiration

Maximum respiration

Proton leak
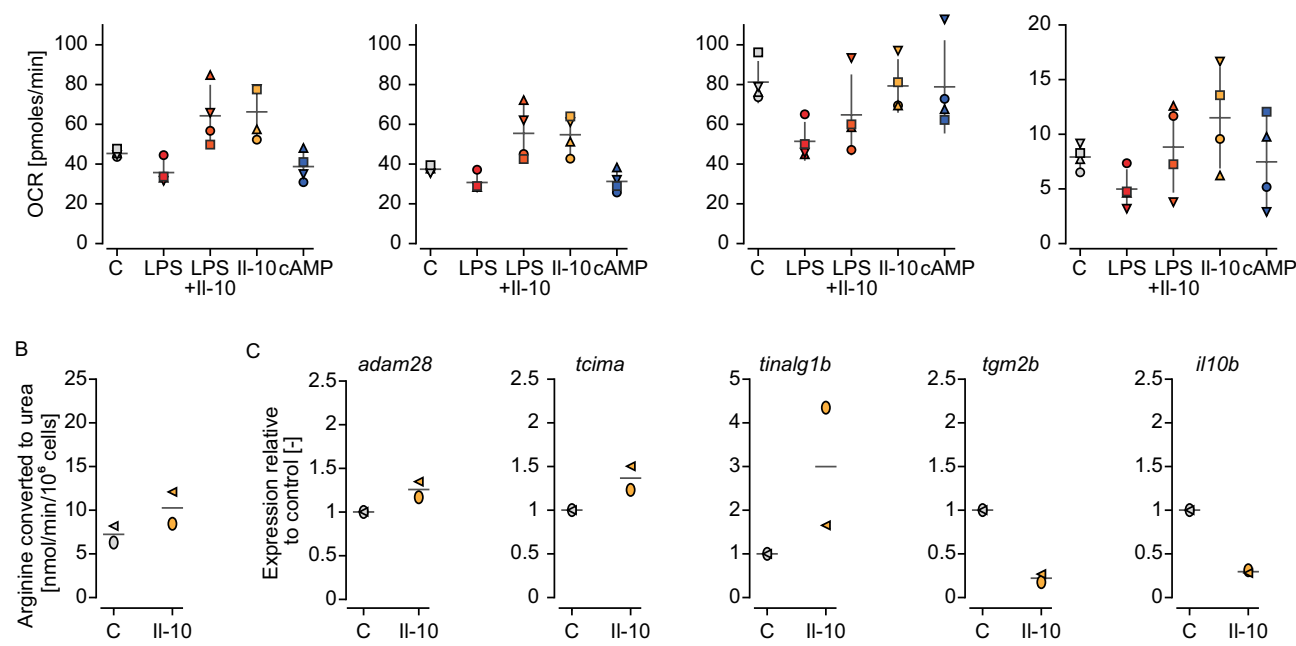

Figure 6.6 Stimulation of macrophages with IL-10 alone shows modest upregulation of arginase activity and upregulation of some M2 marker genes and downregulation of some others. A) Head kidney-derived macrophages were stimulated with $20 \mu \mathrm{g} / \mathrm{ml} \mathrm{LPS}$ (red), $10 \mu \mathrm{g} / \mathrm{ml}$ Il-10 (yellow), $0.5 \mu \mathrm{g} /$ ml cAMP (blue) or a combination of LPS and Il-10 (orange) for $24 \mathrm{~h}$ or kept as unstimulated controls (grey). Oxygen consumption rate (OCR)-derived parameters (Basal respiration, ATP-linked respiration, Maximum respiration and Proton leak) were measured and calculated as described in chapter 6. B) Macrophages were stimulated with $10 \mu \mathrm{g} / \mathrm{ml} \mathrm{Il-10} \mathrm{(yellow)} \mathrm{for} 24 \mathrm{~h}$ or kept as unstimulated controls (grey) to determine arginase activity. C) Macrophages were stimulated for $6 \mathrm{~h}$ to determine relative gene expression by RT-qPCR of proposed M2 markers identified in chapter 3 (adam28, tcima, tinagl1b or $\operatorname{tgm} 2 b$ ) or to determine relative expression of $i l 10 b$. All data are presented as means (horizontal line), standard deviation (A) and as individual fish (symbols). 
Cortisol, similar to Il-10, can reduce pro-inflammatory responses of monocytes/ macrophages at least when freshly isolated from the head kidney of carp. These include a downregulation of LPS-induced expression of il12p35 and il1b [40], although without accompanying upregulation of il10 or arg2. Interestingly, expression of a number of candidate M2 markers increased in Il-10 stimulated macrophages can be seen similarly regulated in cortisol-stimulated monocytes/ macrophages from carp (Figure 6.7). This includes moderate but significant upregulation of adam 28 , tcima, tinagl $1 b$ and $\operatorname{mrc} 1 b$ while timp $2 b$ and $\operatorname{tgm} 2 b$ remain unchanged. Although this cell population cannot be directly compared with the in vitro cultured macrophages studied throughout this thesis, these preliminary findings indicate that cortisol induces a phenotype which may be more similar to the Il-10-induced phenotype (M2c-like?) than to the more 'traditional' cAMPinduced M2(a) phenotype. No matter what, our data suggest that anti-inflammatory phenotypes of carp macrophages could indeed be broader than only the cAMPinduced M2(a) phenotype.
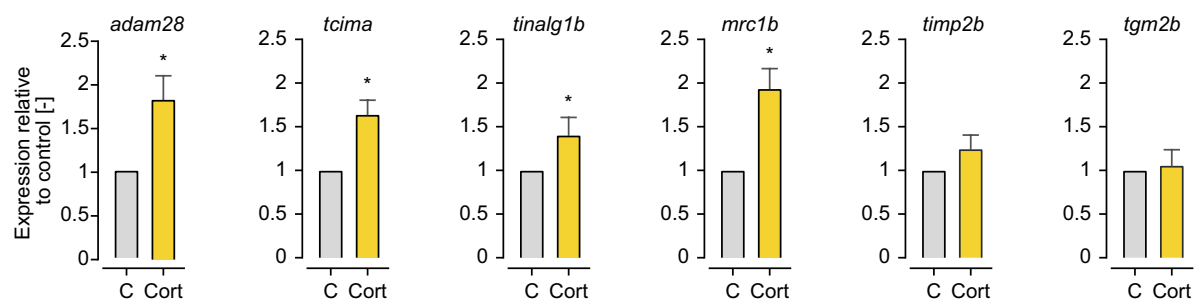

Figure 6.7 Monocytes/macrophages from carp head kidney show modest upregulation of some M2 marker genes. Head kidney monocytes/macrophages were isolated with a percoll gradient $(1.060 \mathrm{~g} /$ $\mathrm{cm}^{3}$ layer) and stimulated for $6 \mathrm{~h}$ with $1 \mu \mathrm{M}$ cortisol (yellow) or kept as unstimulated controls (grey) to determine relative gene expression by RT-qPCR of proposed M2 markers identified in chapter 3 (adam28, tcima, tinagl $1 b$ or tgm $2 b$ ). All data are presented as means and standard deviation. Asterisks (*) indicate significant differences $(p<0.05 \text {, ANOVA, followed by Tukey's post hoc test })^{1 .}$

\section{From single stimuli to complex pathogens: how do macrophage profiles changes during infection}

In vivo, e.g. during infections, macrophages encounter much more complex microenvironments than can be mimicked in vitro. These microenvironments are comprised of (soluble factors secreted by) various local cell types and influenced

Data and analysis obtained from Magdalena Chadzińska, Department of Evolutionary Immunology, Institute of Zoology and Biomedical Research, Jagiellonian University, Krakow, Poland 
by recruited immune cells. It is safe to assume there is complex signaling with a vast number of cytokines, chemokines, growth factors, lipid mediators such as prostaglandins and cell-cell interactions that can all influence the macrophage phenotype. As such, it is of interest to study whether the polarized macrophage profiles based on our in vitro approach could be valuable also as read out for in vivo situations. To examine this, we studied transcriptome sets of carp tissues collected after experimental infection with different microbes.

First, we studied spleen of carp infected with Cyprinid Herpes Virus-3 (CyHV-3) [52]. When looking for candidate M1 and M2 markers in CyHV-3-infected spleen, we noticed patterns of regulation that were predominantly hinting at the presence of M1 macrophages: from 1135 genes significantly upregulated after virus infection, 12 were previously proposed as M1 markers (Figure 6.8). Interestingly, among these genes were slc2a6 and mecr, related to cellular energy metabolism. Of the 12 M1 markers upregulated with CyHV-3, four genes were even > $1.5 \log 2$ fold upregulated and include tissue factor $3(f 3 b)$ and complement factors ( $c d b$ / c2-a3). Moreover, 1 more gene (cxcl11-like or cxcl18b) normally downregulated in M2 macrophages also was $1.5 \log 2$ fold upregulated with CyHV-3. Of the 460 genes significantly downregulated with CyHV-3 most overlapped with markers normally up- and not downregulated in M2 macrophages. Although isolation of macrophages from infected tissues would be necessary to ascertain whether in vivo these genes were expressed by macrophages, our analysis after this viral infection indicates a tipped balance towards an environment which would favor the presence of M1 inflammatory macrophages.

Second, we studied skin of carp infected with the protozoan parasite Ichthyophthirius multifiliis (Ich) ${ }^{2}$. When looking for candidate M1 and M2 markers in Ich-infected skin we were slightly restricted by the dataset with only 2 replicates per time point and thus used $p<0.05$ instead of the corrected $p_{\text {adjusted }}$ for further subsetting after DESeq2 analysis [33]. Only few candidate markers were upregulated after infection with Ich, none of which seemed stable over 5 days post-infection and none seemed part of a clear and consistent pattern rather than a singular upregulated gene. On the other hand, we discovered five M1 markers simultaneously downregulated in Ich-infected skin at 1 day after infection. These markers included two hsp70, two saa

$\overline{2}$ Samples obtained by Csaba and Boglarka, Centre for Agricultural Research, Budapest, Hungary 

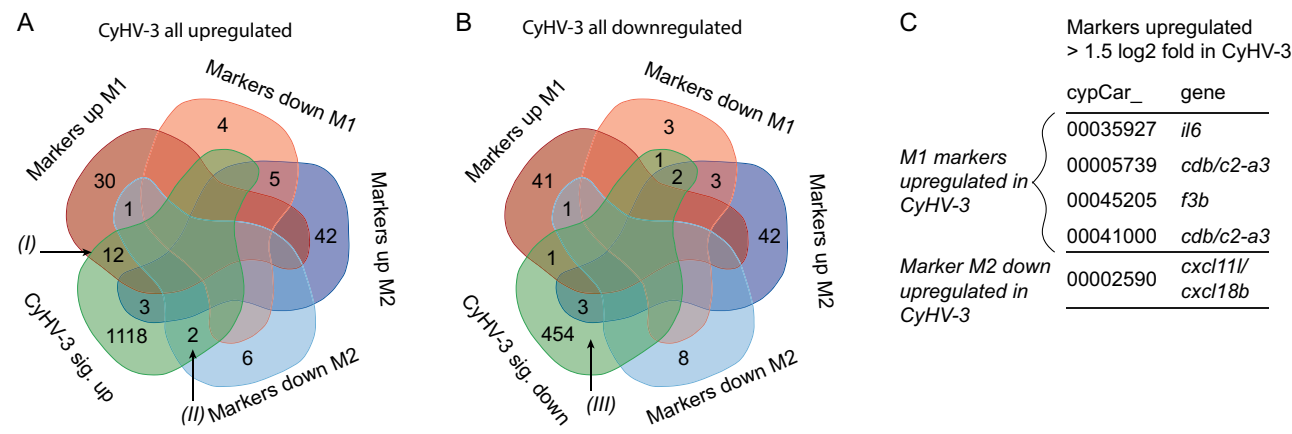

Figure 6.8 Venn diagrams of candidate M1 and M2 markers show the presence of predominantly M1 markers in whole spleen transcriptomes of CyHV-3 infected carp. Predicted genes significantly $\left(p_{\text {adiusted }}=0.05\right.$, analyzed by DESeq2 [33]) upregulated (A) or downregulated (B) in Cyprinid Herpes Virus-3 (CyHV-3) infected spleen of susceptible carp 4 days after infection. Predicted genes are cross-referenced against proposed markers for M1 and M2 macrophages depicted in dark red (M1 up), light red (M1 down), dark blue (M2 up) and light blue (M2 down. Upregulated genes in CyHV-3 infected spleens predominantly overlap with upregulated M1 markers (I) or downregulated M2 markers (II). Downregulated genes in CyHV3 predominantly overlap with downregulated M2 markers (III). C) Markers highly (log2 fold > 1.5) upregulated in CyHV-3 infected spleens. CypCar numbers represent predicted genes [29].

and one complement factor $b(c f b)$ gene, which could suggest a more M2-like profile early during infection. Interestingly, there also appears to be a downregulation of expression of the Il-13 receptor alpha (il13ra1) 5 days post-infection, which may indicate a negative feedback mechanism at a later time point. These very preliminary data indicate that experimental infection with the protozoan Ich may induce a macrophage immune phenotype in skin of anti-inflammatory nature. Examination of macrophages in infected tissue by immunohistochemical or in situ hybridization approaches could possibly provide more detailed information on their polarization state. Taken together, although the exact cell type expressing these markers remain undetermined in such studies, we are confident our panel of candidate markers can provide a useful mean to establish the likely presence of (anti-) inflammatory macrophages in tissue samples collected post-infection.

\section{Can profiling of metabolism replace profiling of immune gene expression?}

In recent decades immunologists have increasingly realized the importance of cellular metabolism for the function of immune cells including lymphocytes and macrophages. For macrophages, the differential metabolism of the amino acid L-arginine has been on the radar for quite some time [53] and since then has been considered characteristic for polarized macrophages of multiple species, including some fish species [23, 54]. In more recent years studies into the role of 
different metabolic processes supporting the functional phenotype of immune cells have expanded into the combined field of immunometabolism [55]. For polarized macrophages, studies predominantly performed in mice have discovered a metabolic switch that occurs in M1 macrophages, which drives them to change energy source from oxidative phosphorylation to glycolysis [56-58]. Further studies shed light on the close connection between energy metabolism and immunity (see also chapter 6). Interestingly, a number of the marker genes we confirmed being highly expressed in inflammatory macrophages are related to macrophage metabolic reprogramming.

Among these are the metabolic genes slc2a6, irg1, inos (nos2b) and mecr (Figure 6.9, see also Figure 6.1). In mice, slc2a6 is also known as glut6 and encodes a lysosomal transporter that stimulates glycolysis in macrophages, is regulated by the NF- $\mathrm{kb}$ pathway and shows increased expression upon stimulation with different TLR ligands [59]. The gene irg1 codes for a protein that catalyzes the production of itaconate which is considered strongly involved in metabolic reprogramming of macrophages. Increased intracellular citrate concentration contributes to the production of $\mathrm{NO}$ and the production of fatty acids, lipids and prostaglandins. The gene inos (nos $2 b$ ) is ultimately responsible for the production of NO, which in turn inhibits the electron transport chain and thus contributes to increased levels of intracellular succinate, Hif1 $\alpha$ stabilization and ROS production. Finally, the switch from fatty acid metabolism to glycolysis allows for fatty acid synthesis and mecr is involved in mitochondrial fatty acid synthesis [60]. The detection of these metabolic markers not only in vitro (Figure 6.9) but also in vivo (slc2a6 and mecr) once again highlights the high degree of conservation of metabolic pathways, at least the one activated in inflammatory macrophages. This conservation of metabolism seems to include conservation between species and conservation between different inflammatory stimuli. Certainly, immunometabolism provides an interdisciplinary route to study conserved polarization of innate immune cells such as macrophages.

Our results in chapter 6 suggest a relatively tight regulation of oxygen consumption increase in carp M2 macrophages. Oxygen saturation in water is lower than air, which may cause the cellular metabolism of fish to be geared to oxygen more sparingly then mammals. Indeed, basal oxygen use of many fish species, including carp, is $50-100 \mathrm{x}$ lower than that of mammals or birds [62]. A more lenient use of oxygen by mammalian immune cells could be the facilitated by adaptations that 
evolved to meet increased oxygen demands for thermal regulation of endotherms. Of particular interest in cold-blooded aquatic species such as carp, is the potential influence of environmental factor such as temperature and oxygen availability on (immuno)metabolism and polarization. In fact, temperature is of direct influence on ADP affinity in the mitochondria (reviewed by [63]) and on oxygen saturation of the water [64]. Especially for fish species such as the common carp that tolerate a large temperature range with correlated differ ences in different oxygen saturation it may be of interest to study how these environmental parameters affect the (immuno)metabolism of innate immune cells such as macrophages. A route not yet explored is measuring macrophage polarization in tissue specific populations, as those residing in the gills' respiratory tissue, where immune cells may be directly influenced by local fluctuations in oxygen availability.

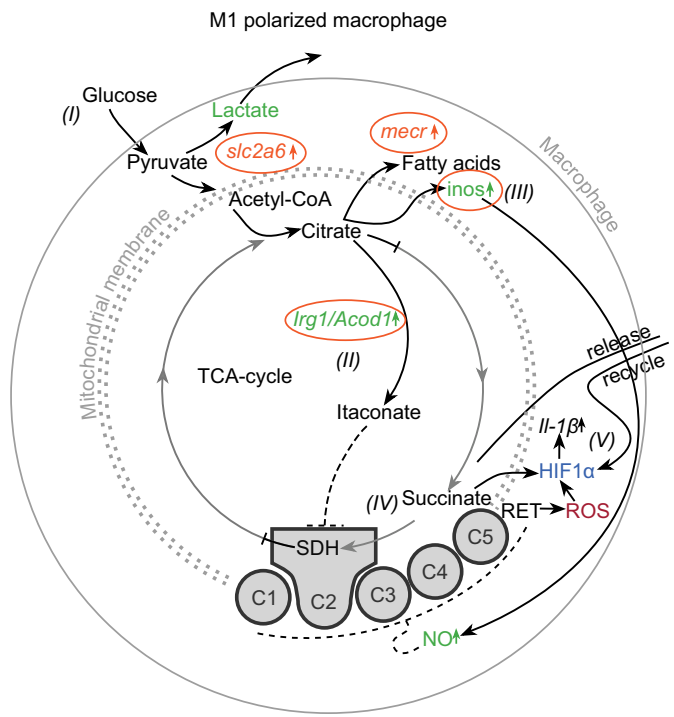

Figure 6.9 Inflammatory macrophage markers can be nearly directly linked to metabolism. Schematic representation of metabolic reprogramming towards glycolysis in murine macrophages, but modified for carp M1 macrophages and with inflammatory marker genes either added or circled in the appropriate pathways in orange. Pathways in black are enhanced and pathways in grey with dashed lines are decreased in murine macrophages. Text in green refers to intermediates with regulation in LPS-stimulated carp macrophages similar to regulation in mice. Text in blue refers to intermediates present in zebrafish but not yet studied in carp. Text in red refers to mechanisms present in carp M1 macrophages but not regulated similar to those in mice. Figure based on Van den Bossche et al., 2017.

Regulation of cell metabolism potentially may be one of the most conserved traits of innate immune responses throughout evolution. Cellular energy metabolism involves processes that are highly conserved across species, as illustrated by the conservation of the glycolytic and aerobic respiration + tricarboxylic acid (TCA)-cycle pathways in all three domains: Archaea, Bacteria and Eucarya [65]. Immunometabolic changes under polarizing conditions are not limited to macrophages but are driven by a need for resources in many different cell types 
(reviewed in [55]). With this in mind, measuring metabolic parameters may be considered one of the most straightforward routes for quantifying polarization of innate immune responses all the way down to invertebrate species including insects, shellfish, starfish and many more. Last but not least, studying macrophage polarization in invertebrates typically without adaptive immunity may provide further experimental support to the 'macrophage first' hypothesis.

\section{Plasticity of macrophage responses}

The inevitable question that many macrophage biologists face and which has occupied the macrophage field for years is whether or not macrophages once polarized, can re-polarize. Our results indicate a possibly relatively flexible phenotype in carp, at least at metabolic level, because LPS-stimulated and thus M1 polarized macrophages showed a reduction in oxygen consumption at basal level early after activation which normalized again after $24 \mathrm{~h}$. If this normalization would not occur in carp M1 macrophages polarized with LPS in combination with Ifn- $\gamma$, it would tell us if Ifn- $\gamma$ is potentially responsible for fate confirmation. Van den Bossche et al. (2016) showed that NO production is responsible for mitochondrial dysfunction in M1 macrophages and prevents effective repolarization towards an M2 phenotype, at least in murine and human macrophages. This could possibly be explained by the interference of NO with iron-sulfur containing complexes in the electron transport chain (ETC) and contribution of NO to blocking of the ETC [6668]. In apparent contrast, a tracking experiment in zebrafish larvae using transgenic $m p e g 1^{+} / t n f a^{+}$macrophages recruited to an injury site showed that this M1 phenotype could be reverted to an intermediate phenotype at later time points [69]. Whether the apparently different outcome is due primarily to the differences in experimental setting or indicates more flexibility in function of polarized macrophages in fish remains to be determined. In all, these macrophage repolarization and NO-induced mitochondrial dysfunction remains to be studied thoroughly in carp macrophages.

This discussion is packed with examples that illustrate an evolutionary conservation of macrophage polarization. The abilities of carp macrophages to polarize into cells with different functional phenotypes, transcriptional profiles and metabolic profiles appear well conserved. With that, we need to mention that although the profiles we observed appear well-conserved, many subsets have only been partly characterized and unanswered questions remain, especially when it comes to inducing M2 polarization and the role of products from fast evolving 
genes like il4/13. Nevertheless, we have observed many subtle differences in expression between macrophages activated with different stimuli that induce pro-inflammatory responses (LPS, LPS + Ifn- $\gamma$ or zymosan) or different stimuli that induce anti-inflammatory responses (cAMP, PGE2, Il-10 or cortisol). Although we cannot exclude for all stimuli that these differences could be dependent on concentration of the stimulus or timing, the arguments for adopting a spectrum view on macrophage polarization in fish become more and more convincing. We would therefore support adopting a nomenclature for polarized fish macrophages that includes the stimulus as proposed by Murray et al. (2014), without adhering strictly to the phenotype associated with mammalian macrophages induced with the same stimulus (Figure 6.10).

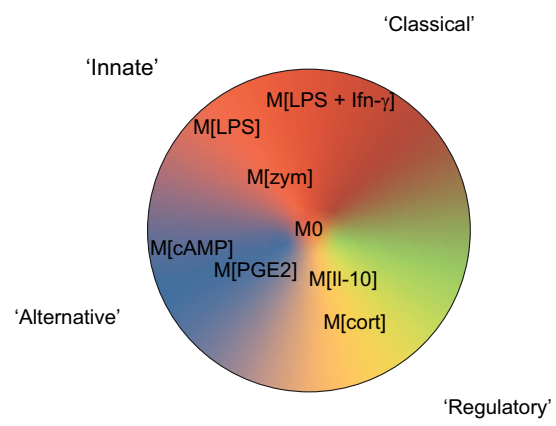

Figure 6.10 Hypothetical spectrum of polarized carp macrophages based on this thesis. Schematic hypothetical overview of polarized macrophage phenotypes examined to different degrees in this thesis including those unstimulated (M0) and stimulated with LPS, LPS + Ifn- $\gamma$, zymosan (zym), cAMP, PGE2, Il-10 and cortisol (cort).

Next to our description of macrophage profiles, with our metabolic analyses we started to define immunometabolism in carp macrophages, which may be one of the most conserved traits of polarized macrophages. Furthermore, we have taken the first steps towards exploring the value of the markers we defined for in vitro situations for in vivo responses following complex stimulation such as viral or parasitic infection. Overall, we have gained important information on the polarized innate immune responses of carp with macrophages as key players, steering innate immune responses to help fish survive in the intense and immunologically challenging environment of aquaculture practice. 


\section{References}

1. Magnadóttir B (2006) Innate immunity of fish (overview). Fish Shellfish Immunol 20:137-151. doi:10.1016/j.fsi.2004.09.006.

2. Danilova N (2012) The Evolution of Adaptive Immunity BT - Self and Nonself. In: López-Larrea C (ed). Springer US, New York, NY, pp 218-235.

3. Ward AE, Rosenthal BM (2014) Evolutionary responses of innate immunity to adaptive immunity. Infect Genet Evol 21:492-496. doi:10.1016/j.meegid.2013.12.021.

4. Netea MG, Schlitzer A, Placek K, Joosten LAB, Schultze JL (2019) Innate and Adaptive Immune Memory: an Evolutionary Continuum in the Host's Response to Pathogens. Cell Host Microbe 25:13-26. doi:10.1016/j.chom.2018.12.006.

5. Abram QH, Dixon B, Katzenback BA (2017) Impacts of Low Temperature on the Teleost Immune System. Biology (Basel) 6:39. doi:10.3390/biology6040039.

6. Magor BG, Magor KE (2001) Evolution of effectors and receptors of innate immunity. Dev Comp Immunol 25:651-682. doi:10.1016/S0145-305X(01)00029-5.

7. Mills CD, Ley K (2014) M1 and M2 Macrophages : The Chicken and the Egg of Immunity. J Innate Immun 6:716-726. doi:10.1159/000364945.

8. Dougan M, Dranoff G, Dougan SK (2019) GM-CSF, IL-3, and IL-5 Family of Cytokines: Regulators of Inflammation. Immunity 50:796-811. doi:10.1016/j.immuni.2019.03.022

9. Ushach I, Zlotnik A (2016) Biological role of granulocyte macrophage colony-stimulating factor (GM-CSF) and macrophage colony-stimulating factor (M-CSF) on cells of the myeloid lineage. J Leukoc Biol 100:481-489. doi:10.1189/jlb.3RU0316-144R.

10. Yamaguchi T, Takizawa F, Fischer U, Dijkstra J (2015) Along the Axis between Type 1 and Type 2 Immunity; Principles Conserved in Evolution from Fish to Mammals. Biology (Basel) 4:814-859. doi:10.3390/biology4040814.

11. Yang K, Feng S, Zhang S, Yin L, Zhou H, Zhang A, Wang X (2019) Characterization of a new il-4/13 homologue in grass carp (Ctenopharyngodon idella) and its cooperation with M-CSF to promote macrophage proliferation. Fish Shellfish Immunol 93:508-516. doi:10.1016/j. fsi.2019.07.070.

12. Wang T, Secombes CJ (2015) The evolution of IL-4 and IL-13 and their receptor subunits. Cytokine 75:8-13. doi:10.1016/j.cyto.2015.04.012.

13. Ohtani M, Hayashi N, Hashimoto K, Nakanishi T, Dijkstra JM (2008) Comprehensive clarification of two paralogous interleukin 4/13 loci in teleost fish. Immunogenetics 60:383-397. doi:10.1007/ s00251-008-0299-x.

14. Dijkstra JM (2014) TH 2 and Treg candidate genes in elephant shark. Nature 511:E7-E9. doi:10.1038/nature13446.

15. Stocchi V, Wang T, Randelli E, Mazzini M, Gerdol M, Pallavicini A, Secombes CJ, Scapigliati G, Buonocore F (2017) Evolution of Th2 responses: characterization of IL-4/13 in sea bass (Dicentrarchus labrax L.) and studies of expression and biological activity. Sci Rep 7:2240. doi:10.1038/s41598-017-02472-y. 
16. Ohno S (1970) Nature's Great Experiment with Gene Duplication during Evolution from Tunicate-like Creatures to Fish BT - Evolution by Gene Duplication. In: Ohno S (ed). Springer Berlin Heidelberg, Berlin, Heidelberg, pp 124-132.

17. Force A, Lynch M, Pickett FB, Amores A, Yan Y, Postlethwait J (1999) Preservation of Duplicate Genes by Complementary, Degenerative Mutations. Genetics 151:1531 LP - 1545..

18. Cresko WA, Yan Y-L, Baltrus DA, Amores A, Singer A, Rodríguez-Marí A, Postlethwait JH (2003) Genome duplication, subfunction partitioning, and lineage divergence: Sox9 in stickleback and zebrafish. Dev Dyn 228:480-489. doi:10.1002/dvdy.10424.

19. Woolfe A, Elgar G (2007) Comparative genomics using Fugu reveals insights into regulatory subfunctionalization. Genome Biol 8:R53. doi:10.1186/gb-2007-8-4-r53.

20. Li J-T, Hou G-Y, Kong X-F, Li C-Y, Zeng J-M, Li H-D, Xiao G-B, Li X-M, Sun X-W (2015) The fate of recent duplicated genes following a fourth-round whole genome duplication in a tetraploid fish, common carp (Cyprinus carpio). Sci Rep 5:8199. doi:10.1038/srep08199.

21. Boldajipour B, Doitsidou M, Tarbashevich K, Laguri C, Yu SR, Ries J, Dumstrei K, Thelen S, Dörries J, Messerschmidt E-M, Thelen M, Schwille P, Brand M, Lortat-Jacob H, Raz E (2011) Cxcl12 evolution - subfunctionalization of a ligand through altered interaction with the chemokine receptor. Development 138:2909 LP - 2914. doi:10.1242/dev.068379.

22. Lv H, Zhou T, Dong C, Kong S, Chen L, Pu F, Li X, Xu P (2020) Genome-wide identification, evolution, and mRNA expression of complement genes in common carp (Cyprinus carpio). Fish Shellfish Immunol 96:190-200. doi:https://doi.org/10.1016/j.fsi.2019.11.032.

23. Hodgkinson JW, Fibke C, Belosevic M (2017) Recombinant IL-4/13A and IL-4/13B induce arginase activity and down-regulate nitric oxide response of primary goldfish (Carassius auratus L.) macrophages. Dev Comp Immunol 67:377-384. doi:10.1016/j.dci.2016.08.014.

24. Yamaguchi T, Miyata S, Katakura F, Nagasawa T, Shibasaki Y, Yabu T, Fischer U, Nakayasu C, Nakanishi T, Moritomo T (2016) Recombinant carp IL-4/13B stimulates in vitro proliferation of carp IgM+ B cells. Fish Shellfish Immunol 49:225-229. doi:10.1016/J.FSI.2015.12.043.

25. David L, Blum S, Feldman MW, Lavi U, Hillel J (2003) Recent Duplication of the Common Carp (Cyprinus carpio L.) Genome as Revealed by Analyses of Microsatellite Loci. Mol Biol Evol 20:1425-1434. doi:10.1093/molbev/msg173.

26. Wang J-T, Li J-T, Zhang X-F, Sun X-W (2012) Transcriptome analysis reveals the time of the fourth round of genome duplication in common carp (Cyprinus carpio). BMC Genomics 13:96. doi:10.1186/1471-2164-13-96.

27. Xu P, Xu J, Liu G, Chen L, Zhou Z, Peng W, Jiang Y, Zhao Z, Jia Z, Sun Y, Wu Y, Chen B, Pu F, Feng J, Luo J, Chai J, Zhang H, Wang H, Dong C, Jiang W, Sun X (2019) The allotetraploid origin and asymmetrical genome evolution of the common carp Cyprinus carpio. Nat Commun 10:4625. doi:10.1038/s41467-019-12644-1.

28. Petit J, David L, Dirks R, Wiegertjes GF (2017) Genomic and transcriptomic approaches to study immunology in cyprinids: What is next? Dev Comp Immunol 75:48-62. doi:10.1016/j. dci.2017.02.022. 
29. Kolder ICRM, van der Plas-Duivesteijn SJ, Tan G, Wiegertjes GF, Forlenza M, Guler AT, Travin DY, Nakao M, Moritomo T, Irnazarow I, den Dunnen JT, Anvar SY, Jansen HJ, Dirks RP, Palmblad M, Lenhard B, Henkel C V, Spaink HP (2016) A full-body transcriptome and proteome resource for the European common carp. BMC Genomics 17:701. doi:10.1186/s12864-016-3038-y.

30. Yamaguchi T, Katakura F, Someya K, Dijkstra JM, Moritomo T, Nakanishi T (2013) Clonal growth of carp (Cyprinus carpio) T cells in vitro: Long-term proliferation of Th2-like cells. Fish Shellfish Immunol 34:433-442. doi:10.1016/j.fsi.2012.11.005.

31. Underhill D (2003) Macrophage recognition of zymosan particles. J Endotoxin Res 9:176-180. doi:10.1179/096805103125001586.

32. Petit J, Bailey EC, Wheeler RT, de Oliveira CAF, Forlenza M, Wiegertjes GF (2019) Studies Into $\beta$-Glucan Recognition in Fish Suggests a Key Role for the C-Type Lectin Pathway. Front Immunol 10:280.

33. Love MI, Huber W, Anders S (2014) Moderated estimation of fold change and dispersion for RNA-seq data with DESeq2. Genome Biol 15:550. doi:10.1186/s13059-014-0550-8.

34. Vignali DAA, Kuchroo VK (2012) IL-12 family cytokines: immunological playmakers. Nat Immunol 13:722-728. doi:10.1038/ni.2366.

35. Wang T, Husain M (2014) The expanding repertoire of the IL-12 cytokine family in teleost fish: Identification of three paralogues each of the $\mathrm{p} 35$ and p 40 genes in salmonids, and comparative analysis of their expression and modulation in Atlantic salmon Salmo salar. Dev Comp Immunol 46:194-207. doi:10.1016/j.dci.2014.04.008.

36. Martinez FO, Gordon S (2014) The M1 and M2 paradigm of macrophage activation : time for reassessment. F1000 Prime Reports 6-13. doi:10.12703/P6-13.

37. Murray PJ, Allen JE, Biswas SK, Fisher EA, Gilroy DW, Goerdt S, Gordon S, Hamilton JA, Ivashkiv LB, Lawrence T, Locati M, Mantovani A, Martinez FO, Mege J-L, Mosser DM, Natoli G, Saeij JP, Schultze JL, Shirey KA, Sica A, Suttles J, Udalova I, van Ginderachter JA, Vogel SN, Wynn TA (2014) Macrophage activation and polarization: nomenclature and experimental guidelines. Immunity 41:14-20. doi:10.1016/j.immuni.2014.06.008.

38. Piazzon MC, Savelkoul HFJ, Pietretti D, Wiegertjes GF, Forlenza M (2015) Carp Il10 Has Anti-Inflammatory Activities on Phagocytes, Promotes Proliferation of Memory T Cells, and Regulates B Cell Differentiation and Antibody Secretion. J Immunol 194:187-199. doi:10.4049/ jimmunol.1402093.

39. Piazzon MC, Wentzel AS, Wiegertjes GF, Forlenza M (2017) Carp Il10a and Il10b exert identical biological activities in vitro, but are differentially regulated in vivo. Dev Comp Immunol 67:350360. doi:10.1016/j.dci.2016.08.016.

40. Maciuszek M, Rydz L, Świtakowska I, Verburg-van Kemenade BML, Chadzińska M (2019) Effects of stress and cortisol on the polarization of carp macrophages. Fish Shellfish Immunol 94:27-37. doi:10.1016/j.fsi.2019.08.064.

41. Luan B, Yoon Y-S, Le Lay J, Kaestner KH, Hedrick S, Montminy M (2015) CREB pathway links PGE2 signaling with macrophage polarization. Proc Natl Acad Sci 112:15642 LP - 15647. doi:10.1073/pnas.1519644112. 
42. Aronoff DM, Canetti C, Serezani CH, Luo M, Peters-Golden M (2005) Cutting Edge: Macrophage Inhibition by Cyclic AMP (cAMP): Differential Roles of Protein Kinase A and Exchange Protein Directly Activated by cAMP-1. J Immunol 174:595 LP - 599. doi:10.4049/jimmunol.174.2.595.

43. Corraliza IM, Soler G, Eichmann K, Modolell M (1995) Arginase Induction by Suppressors of Nitric Oxide Synthesis (IL-4, IL-10 and PGE2) in Murine Bone-Marrow-Derived Macrophages. Biochem Biophys Res Commun 206:667-673. doi:10.1006/bbrc.1995.1094.

44. Csóka B, Selmeczy Z, Koscsó B, Németh ZH, Pacher P, Murray PJ, Kepka-Lenhart D, Morris Jr SM, Gause WC, Leibovich SJ, Haskó G (2012) Adenosine promotes alternative macrophage activation via A2A and A2B receptors. FASEB J 26:376-386. doi:10.1096/fj.11-190934.

45. Murakami M, Kudo I (2004) Recent advances in molecular biology and physiology of the prostaglandin E2-biosynthetic pathway. Prog Lipid Res 43:3-35. doi:10.1016/S01637827(03)00037-7.

46. Osma-Garcia IC, Punzón C, Fresno M, Díaz-Muñoz MD (2016) Dose-dependent effects of prostaglandin E2 in macrophage adhesion and migration. Eur J Immunol 46:677-688. doi:10.1002/eji.201545629.

47. Na YR, Jung D, Yoon BR, Lee WW, Seok SH (2015) Endogenous prostaglandin E2 potentiates anti-inflammatory phenotype of macrophage through the CREB-C/EBP- $\beta$ cascade. Eur $J$ Immunol 45:2661-2671. doi:10.1002/eji.201545471.

48. Tang T, Scambler TE, Smallie T, Cunliffe HE, Ross EA, Rosner DR, O’Neil JD, Clark AR (2017) Macrophage responses to lipopolysaccharide are modulated by a feedback loop involving prostaglandin E2, dual specificity phosphatase 1 and tristetraprolin. Sci Rep 7:4350. doi:10.1038/ s41598-017-04100-1.

49. Knight J, Lloyd-Evans R, Rowley AF, Barrow SE (1993) Effect of lipoxins and other eicosanoids on phagocytosis and intracellular calcium mobilisation in rainbow trout (Oncorhynchus mykiss) leukocytes. J Leukoc Biol 54:518-522. doi:10.1002/jlb.54.6.518.

50. Novoa B, Figueras A, Ashton I, Secombes CJ (1996) In vitro studies on the regulation of rainbow trout (Oncorhynchus mykiss) macrophage respiratory burst activity. Dev Comp Immunol 20:207-216. doi:10.1016/0145-305X(96)00011-0.

51. Montero J, Gómez-Abellán V, Arizcun M, Mulero V, Sepulcre MP (2016) Prostaglandin E2 promotes M2 polarization of macrophages via a cAMP/CREB signaling pathway and deactivates granulocytes in teleost fish. Fish Shellfish Immunol 55:632-641. doi:10.1016/j.fsi.2016.06.044.

52. Tadmor-Levi R, Doron-Faigenboim A, Marcos-Hadad E, Petit J, Hulata G, Forlenza M, Wiegertjes GF, David L (2019) Different transcriptional response between susceptible and resistant common carp (Cyprinus carpio) fish hints on the mechanism of CyHV-3 disease resistance. BMC Genomics 20:1019. doi:10.1186/s12864-019-6391-9.

53. Mills CD, Kincaid K, Alt JM, Heilman MJ, Hill AM (2000) M-1/M-2 Macrophages and the Th1/ Th2 Paradigm. J Immunol. https://doi.org/10.4049/jimmunol.164.12.6166.

54. Joerink M, Ribeiro CMS, Stet RJM, Hermsen T, Savelkoul HFJ, Wiegertjes GF (2006) Head Kidney-Derived Macrophages of Common Carp (Cyprinus carpio L.) Show Plasticity and Functional Polarization upon Differential Stimulation. J Immunol 177:61-69. doi:10.4049/ jimmunol.177.1.61. 
55. O’Neill LAJ, Kishton RJ, Rathmell J (2016) A guide to immunometabolism for immunologists. Nat Rev Immunol 16:553. doi: 10.1038/nri.2016.70.

56. Van den Bossche J, Baardman J, de Winther MPJ (2015) Metabolic Characterization of Polarized M1 and M2 Bone Marrow-derived Macrophages Using Real-time Extracellular Flux Analysis. J Vis Exp 53424. doi:10.3791/53424.

57. Van den Bossche J, Baardman J, Otto NA, van der Velden S, Neele AE, van den Berg SM, LuqueMartin R, Chen H-J, Boshuizen MCS, Ahmed M, Hoeksema MA, de Vos AF, de Winther MPJ (2016) Mitochondrial Dysfunction Prevents Repolarization of Inflammatory Macrophages. Cell Rep 17:684-696. doi:10.1016/j.celrep.2016.09.008.

58. Vijayan V, Pradhan P, Braud L, Fuchs HR, Gueler F, Motterlini R, Foresti R, Immenschuh S (2019) Human and murine macrophages exhibit differential metabolic responses to lipopolysaccharide - A divergent role for glycolysis. Redox Biol 22:101147. doi:10.1016/j.redox.2019.101147.

59. Maedera S, Mizuno T, Ishiguro H, Ito T, Soga T, Kusuhara H (2019) GLUT6 is a lysosomal transporter that is regulated by inflammatory stimuli and modulates glycolysis in macrophages. FEBS Lett 593:195-208. doi:10.1002/1873-3468.13298.

60. Miinalainen IJ, Chen Z-J, Torkko JM, Pirilä PL, Sormunen RT, Bergmann U, Qin Y-M, Hiltunen JK (2003) Characterization of 2-Enoyl Thioester Reductase from Mammals: AN ORTHOLOG OF Ybr026p/Mrf1'p OF THE YEAST MITOCHONDRIAL FATTY ACID SYNTHESIS TYPE II . J Biol Chem 278:20154-20161. doi:10.1074/jbc.M302851200.

61. Van den Bossche J, O’Neill LA, Menon D (2017) Macrophage Immunometabolism: Where Are We (Going)? Trends Immunol 38:395-406. doi:10.1016/j.it.2017.03.001.

62. Brett JR (1972) The metabolic demand for oxygen in fish, particularly salmonids, and a comparison with other vertebrates. Respir Physiol 14:151-170. doi:10.1016/00345687(72)90025-4.

63. O’Brien KM (2011) Mitochondrial biogenesis in cold-bodied fishes. J Exp Biol 214:275 LP - 285. doi:10.1242/jeb.046854.

64. Wetzel RG (2001) Limnology: Lake and River Ecosystems.

65. Peregrin-Alvarez JM, Tsoka S, Ouzounis CA (2003) The Phylogenetic Extent of Metabolic Enzymes and Pathways. Genome Res 13:422-427. doi:10.1101/gr.246903.

66. Cleeter MWJ, Cooper JM, Darley-Usmar VM, Moncada S, Schapira AH V (1994) Reversible inhibition of cytochrome c oxidase, the terminal enzyme of the mitochondrial respiratory chain, by nitric oxide. FEBS Lett 345:50-54. doi:10.1016/0014-5793(94)00424-2.

67. Beltrán B, Mathur A, Duchen MR, Erusalimsky JD, Moncada S (2000) The effect of nitric oxide on cell respiration: A key to understanding its role in cell survival or death. Proc Natl Acad Sci U S A. https://doi.org/10.1073/pnas.97.26.14602.

68. Pearce EL, Pearce EJ (2013) Metabolic pathways in immune cell activation and quiescence. Immunity.

69. Nguyen-Chi M, Laplace-Builhe B, Travnickova J, Luz-Crawford P, Tejedor G, Phan QT, DurouxRichard I, Levraud JP, Kissa K, Lutfalla G, Jorgensen C, Djouad F (2015) Identification of polarized macrophage subsets in zebrafish. Elife 4:1-14. doi:10.7554/eLife.07288. 



\section{Summary (English)}

\section{Samenvatting (Nederlands}

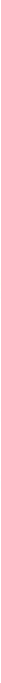




\section{Summary}

Rising demands for animal protein have caused an increase and further intensification of aquaculture over the last decades, which has subsequently led to increased disease pressure. Although preventative vaccines can certainly contribute to the solution, there is also a drive to explore preventative approaches based on immunomodulation of innate immune responses. In either case, it may be clear that a more detailed knowledge of innate immune responses is essential to help combat infectious diseases in aquaculture. Therefore, the overall aim of this thesis is to provide fundamental knowledge of the fish' innate immune system and characterize polarized innate immune responses in carp with the emphasis on macrophages.

In chapter 1 we touch upon the relevance of carp as an aquaculture species and explain why macrophages are considered essential players in innate immune responses, particularly in lower and cold-blooded vertebrates such as fish. We introduce macrophages as cell types that are highly plastic, introduce their activation signals and introduce the concept of macrophage polarization as it has been defined for mammalian macrophages. Then, following a discussion of the framework that will help define macrophage polarization, we briefly summarize existing indications for the presence of polarized macrophages in carp. Finally, we shortly discuss how our findings can aid the development of immunomodulators that could help improve fish health in the context of aquaculture.

We start by thoroughly reviewing the existing literature on macrophage polarization in fish in chapter 2 . We review the stimuli frequently used to polarize macrophages in mammals, as well as the conservation of cytokines often associated with $\mathrm{T}$ helper 1 and $\mathrm{T}$ helper 2 subsets. We discuss approaching macrophage polarization in fish from a 'macrophages first' point of view and consider the plausibility that polarization in fish macrophages could rely primarily on sensing microbial infection or other innate danger signals. Furthermore, we discuss preliminary but promising markers to read out M1 and M2 macrophage responses in fish, including inos as a conserved marker for M1 and arginase 2 as a marker for M2 fish macrophages.

Based on evidence that several key functions of mammalian macrophages are also conserved in fish, we describe comprehensive functional and transcriptional phenotypes of polarized carp macrophages in chapter 3. Here, we combine 
information on established nitric oxide (NO) and arginase assays with morphological differences to first, confirm M1 and M2 macrophage polarization in carp and second, use a sequencing approach to elucidate transcriptional profiles of these M1 and M2 macrophages. We confirm that carp macrophages can polarize into M1- and M2 phenotypes with conserved functions and with transcriptional profiles corresponding to mammalian macrophages. Carp M1 macrophages produce NO and increase expression of pro-inflammatory genes including il6, il12 and saa. Carp M2 macrophages show increased arginase activity and increase expression of anti-inflammatory mediators, including cyr61, timp $2 b$ and tgm $2 b$. Furthermore, we list several candidate markers that can help discriminate between M1 and M2 macrophages of teleost fish. Finally, we touch upon the importance of our findings for the identification of gene targets to generate new transgenic zebrafish for detailed and in vivo studies on M1 and M2 macrophages. Above all, we discuss the striking degree of evolutionary conservation of macrophage polarization in a lower vertebrate.

In chapter 4 we study how M1 macrophages polarized with bacterial lipopolysaccharide (LPS) contribute to neutrophil responses as the major producers of granulocyte colony stimulating factor (Csf3/G-csf). We identify four carp Csf3 paralogs and study basal expression patters in different organs and cell-types. We pinpoint M1 macrophages as the major producers of Csf3 and show that expression is highly increased upon stimulation with mitogens. We characterize both Csf3a and Csf3b as promotors of proliferation in kidney hematopoietic cells, while Csf3b in particular induces neutrophil differentiation. Both CSF3 paralogs were chemotactic for neutrophils and both Csf3a and Csf3b enhance the respiratory burst capacity of neutrophils. The results indicate that M1 macrophage polarization potentiates the proliferation, activation and inflammatory function of neutrophils.

In mammals, M1 macrophages show metabolic reprogramming toward glycolysis, while M2 macrophages rely on oxidative phosphorylation to generate energy. In chapter $\mathbf{5}$, we hypothesize that functional conservation of polarized macrophages in carp could also indicate conservation of associated energy metabolism. Therefore, we study the metabolic profiles of carp macrophages polarized towards M1 and M2 extremes. Using real-time extracellular flux analysis (Seahorse) we determine oxidative phosphorylation and glycolysis in M1 and M2 macrophages. Similar to mammalian M1 macrophages, we found upregulation of irg1 and altered oxidative 
phosphorylation and glycolysis in carp M1 macrophages. In carp M2 macrophages both oxidative phosphorylation and glycolysis were similar to controls. The changes in metabolism of M1 macrophages indicated that metabolic reprogramming may occur in carp M1 macrophages, resulting in distinct metabolic signatures in M1 and M2 carp macrophages. The immunometabolic reprogramming of M1 macrophages likely supports the inflammatory phenotype of these cells in teleost fish such as carp, similar to what has been shown in mammals.

In the General discussion (chapter 6) we discuss our findings primarily in the light of evolutionary conservation of macrophage polarization. We discuss the current knowledge on conservation and use of polarizing cytokines to direct carp macrophages as a next step to expand and refine our understanding of carp macrophage responses. We apply our proposed M1 and M2 markers to elucidate a 'common inflammatory' phenotype and present preliminary results that may indicate that, similar to mammals, the M2 phenotype in fish could include more than just the 'traditional' M2(a) subset. Next, we scrutinize the use of the candidate markers identified in chapter 3 for analysis in tissue collected from in vivo infection studies. We discuss an integrated approach to immunology and metabolism and the potential of immunometabolism as new read-out system for polarized innate immune responses in lower vertebrates. Finally, we discuss the plasticity of fish macrophages and support the adoption of a 'spectrum view' on polarization of fish macrophages.

Overall, in this thesis we describe comprehensive functional phenotypes for M1 and M2 carp macrophages and provide promising candidate markers to study macrophage polarization in carp and possibly also other fish species. We show the ability of polarized M1 macrophages to steer neutrophil responses through the production of Csf3 and indicate that metabolic reprogramming of carp macrophages occurs similar to that in mammalian macrophages. Finally, we discuss future steps to extend our current understanding of polarized innate immune responses in fish with a focus on macrophages and argue in favor of adopting of a spectrum view on macrophage polarization in fish. 


\section{Samenvatting}

Een toenemende vraag naar dierlijke eiwitbronnen heeft geresulteerd in een toename en verdere intensivering van de aquacultuur in de laatste decennia, met als gevolg een verhoogde infectiedruk. Hoewel preventieve vaccins zeker kunnen bijdragen aan het verminderen van infecties, is er ook een motivatie om andere preventieve maatregelen te onderzoeken. Dit zijn bijvoorbeeld benaderingen gebaseerd op modulatie van immuunreactie van het aangeboren immuunsysteem. Ongeacht de beoogde maatregelen, is het duidelijk dat meer gedetailleerde kennis van deze aangeboren immuunreacties essentieel is om infectieuze ziekten in de aquacultuur te bestrijden. Om die reden is het doel van deze thesis om fundamentele kennis van het aangeboren immuunsysteem van de vis te verschaffen en om gepolariseerde aangeboren immuunreacties, met de nadruk op macrofagen, te karakteriseren in de karper.

In hoofdstuk 1 besteden we kort aandacht aan de relevantie van de karper als kweekvis. Daarnaast leggen we uit waarom macrofagen beschouwd worden als essentiële spelers van het aangeboren immuunsysteem, vooral in lagereen koudbloedige gewervelde diersoorten zoals vissen. We introduceren macrofagen als een zeer plastisch celtype, introduceren hun activatiesignalen en introduceren het concept van macrofaagpolarisatie zoals dat is gedefinieerd voor macrofagen van zoogdieren. We zetten het raamwerk uiteen dat ons zal helpen om macrofaagpolarisatie in de vis te definiëren en we vatten kort samen welke indicaties er bestaan voor het optreden van macrofaagpolarisatie in de karper. Als laatste bediscussiëren we kort hoe onze resultaten kunnen helpen om immuunmodulatoren te ontwikkelen die de visgezondheid kunnen verbeteren in de viskweeksector.

Hiervoor beginnen we in hoofdstuk 2 met een grondig onderzoek naar de bestaande literatuur met betrekking tot macrofaagpolarisatie in vissen. We bespreken de stimuli die veelal gebruikt worden om macrofagen van zoogdieren te polariseren en bespreken ook de conservatie van cytokinen die vaak geassocieerd worden met T-helper 1 en T-helper 2 reacties. We bediscussiëren de benadering van macrofaagpolarisatie in vissen vanuit een 'macrophages first' ('macrofagen eerst') perspectief en overwegen we dat het aannemelijk is dat polarisatie van vissenmacrofagen hoofdzakelijk gestuurd wordt door de detectie van microbiële infecties of andere primaire 'gevaarsignalen'. Verder bespreken we preliminaire 
maar veelbelovende markers om M1 en M2 macrofaagreacties te detecteren in vissen. Waaronder inos als geconserveerde marker voor M1 en arginase als geconserveerde marker voor M1 macrofagen in vissen.

Op basis van bewijzen dat enkele belangrijke functies van zoogdiermacrofagen ook voorkomen bij vissen, beschrijven we uitgebreide functionele en transcriptionele fenotypen van gepolariseerde karpermacrofagen in hoofstuk 3. Hier combineren we informatie vanuit gerenommeerde stikstofoxide (nitric oxide, NO) en arginase bepalingen met morfologische verschillen om: als eerste M1 en M2 macrofaagpolarisatie te bevestigen in de karper; en als tweede de transcriptionele profielen van deze M1 en M2 macrofagen op te helderen door middel van RNA sequencing. We kunnen bevestigen dat karpermacrofagen kunnen polariseren in M1- en M2 fenotypen met geconserveerde functies en met transcriptionele profielen die overeenkomen met die van zoogdier macrofagen. M1 karpermacrofagen produceren NO en verhogen de expressie van pro-inflammatoire genen waaronder il6, il12 en saa. M2 karpermacrofagen vertonen verhoogde arginase activiteit en verhogen de expressie van anti-inflammatoire mediatoren waaronder cyr61, timp2b en $\operatorname{tgm} 2 b$. Tevens maken we een opsomming van geschikte kandidaat-markers die kunnen helpen om M1 en M2 macrofagen te onderscheiden in (been)vissen. Als laatste geven we kort aan wat de relevantie is van onze bevindingen voor de identificatie van geschikte doelgenen om nieuwe transgene zebravissen te generen die gebruikt kunnen worden voor gedetailleerde en in vivo studies naar M1 en M2 macrofagen. Bovenal bediscussiëren we de aanzienlijke evolutionaire conservatie van macrofaagpolarisatie in een lagere gewervelde diersoort.

In hoofdstuk 4 bestuderen we hoe M1 macrofagen, gepolariseerd met lipopolysaccharide (LPS), bijdragen aan de reacties van neutrofielen als de primaire producenten van granulocyte colony stimulating factor (Csf3/Gcsf). We identificeren vier Csf3 paralogen in de karper en bestuderen de basale expressiepatronen in verschillende organen en celtypen. We identificeren M1 macrofagen als primaire producenten van Csf3 en laten zien dat de expressie sterk stijgt als gevolg van stimulatie met mitogenen. We karakteriseren zowel Csf3a als Csf3b als promotors van proliferatie in hematopoëtische cellen uit de nier, terwijl met name Csf3b neutrofiel differentiatie induceert. Beide Csf3 paralogen werken chemotactisch op neutrofielen en zowel Csf3a en Csf3b bevorderen de respiratory burst capaciteit van neutrofielen. De resultaten wijzen erop dat M1 
macrofaagpolarisatie de proliferatie, activatie en inflammatoire functie van neutrofielen versterkt.

M1 macrofagen van zoogdieren laten metabole herprogrammering naar glycolyse zien, terwijl M2 macrofagen afhankelijk zijn van oxidatieve fosforylering voor de opwekking van energie. In hoofdstuk $\mathbf{5}$ hypothetiseren we dat de functionele conservatie van macrofaagpolarisatie in karpers erop kan wijzen dat ook het bijbehorende energiemetabolisme geconserveerd is. Daarom bestuderen we de metabole profielen van karpermacrofagen, gepolariseerd naar de M1 en M2 uitersten. We gebruiken real-time extracellulaire flux analyse (Seahorse) om de oxidatieve fosforylering en glycolyse in M1 en M2 macrofagen te bepalen. Vergelijkbaar met M1 macrofagen van zoogdieren vinden we verhoogde expressie van irg1 en aangepaste oxidatieve fosforylering en glycolyse in M1 karpermacrofagen. In M2 karpermacrofagen waren zowel oxidatieve fosforylering als glycolyse vergelijkbaar met de controles. De veranderingen in het metabolisme van M1 macrofagen geeft aan dat metabole herprogrammering wellicht ook plaatsvindt in M1 macrofagen van de karper, wat resulteert verschillende metabole kenmerken in M1 en M2 macrofagen. Dee immuun-metabole herprogrammering van M1 macrofagen ondersteunt naar alle waarschijnlijkheid, net zoals aangetoond in zoogdieren, het inflammatoire fenotype van deze cellen in beenvissen zoals de karper.

In de algemene discussie (hoofstuk 6) bespreken we onze bevindingen met name vanuit het perspectief van de evolutionaire conservatie van macrofaagpolarisatie. We bediscussiëren de huidige kennis over de conservatie en bespreken het gebruik van polariserende cytokinen als volgende stap in het uitbreiden en verfijnen van ons inzicht in de reacties van karpermacrofagen. We passen onze voorgestelde M1 en M2 markers toe om een 'algemeen inflammatoir' fenotype te belichten en kijken vast vooruit met preliminaire resultaten die er wellicht op wijzen dat het M2 fenotype in vissen, net als in zoogdieren, meer behelst dan alleen de traditionele M2(a) subset. Vervolgens onderzoeken we of we de kandidaat-markers uit hoofdstuk 3 kunnen gebruiken voor de analyse van weefsels die zijn verzameld tijdens in vivo infectiestudies. We bespreken een geïntegreerde benadering van immunologie en metabolisme en de potentie van immuunmetabolisme als een nieuwe wijze om gepolariseerde aangeboren immuunreacties uit te lezen in lagere gewervelde diersoorten. Als laatste bespreken we de plasticiteit van vissenmacrofagen en 
onderschrijven we de toepassing van een 'spectrumvisie' op de polarisatie van vissenmacrofagen.

Samengevat, beschrijven we in deze thesis uitgebreide functionele fenotypes van M1 en M2 macrofagen en leveren we veelbelovende kandidaat-markers aan om macrofaagpolarisatie in karpers en wellicht ook andere vissoorten te bestuderen. We laten zien dat gepolariseerde M1 macrofagen het vermogen hebben om reacties van neutrofielen aan te sturen door middel van de productie van Csf3 en we geven aan dat metabole herprogrammering van karpermacrofagen plaatsvindt op een manier die vergelijkbaar is met die in zoogdiermacrofagen. Als laatste bespreken we de toekomstige stappen die nodig zijn om onze huidige inzichten in gepolariseerde aangeboren immuunreacties, met een focus op macrofagen, verder uit te bereiden en pleiten we voor het toepassen van een 'spectrumvisie' als het aankomt op de polarisatie van vissenmacrofagen. 
Summaries 



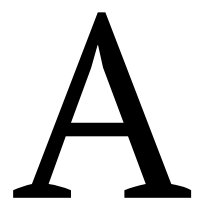

About the author

List of publications

Overview of completed training activities

Acknowledgements 


\section{About the author}

Annelieke Simone Wentzel was born on 24 December 1987 in Apeldoorn, the Netherlands. From the day she was born she has always had an inquisitive mind and an interest in books, nature and animals. At De Werkplaats, her primary and secondary school in Bilthoven, she spend all her early mornings and afternoons taking care of the animals at the schools petting zoo. She felt a great responsibility to take care of the world around her and always helped out animals in any way she could. After getting her Athenaeum degree in 2006 she left home to live and study

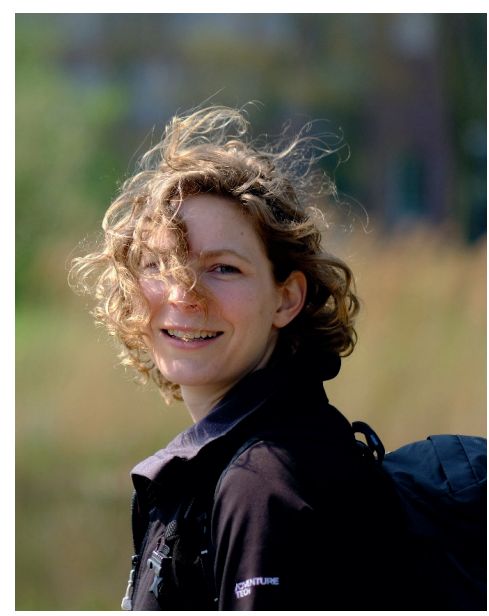
in Leeuwarden. She completed her bachelor's degree in animal management at the Van Hall Institute of Applied Sciences while simultaneously obtaining her paraveterinary degree. After working hours she was running the grand cafe at Van Hall and she participated in many committees of her student association.

After finishing her Bachelor's degree in 2011 she worked for two years, primarily as a veterinary technician in a second-line veterinary clinic, but she was eager to learn more and decided to start studying again. Therefore, she enrolled in the Master program Animal Sciences with a major in Applied Zoology at Wageningen University. Her very first course was the Human and veterinary Immunology course, which she liked so much that she decided to focus her thesis on this topic as well. Her major Master thesis was under supervision of Carla Piazzon-de Haro at the Cell Biology and Immunology Group- studying bioactivity and signaling pathways of Cyprinid Herpes virus 3 (CyHV-3) interleukin 10. This thesis, entitled 'Koi Herpes Virus interleukin-10: bioactivity and signaling pathway' was awarded with the UFW-KLV Thesis Award 2014-2015. Following this path in immunology she performed her minor thesis at the Department of Infectious Diseases and Immunology at Utrecht University. Here she studied the effect of adjuvants on local innate immune responses in a mouse model under the supervision of Susan van Aalst. She graduated cum laude from her Master’s Program in June 2015. 
However, her scientific career had only just started then and she was very happy to be able to continue her research by starting her $\mathrm{PhD}$ project at the Cell biology and Immunology group in September 2015 under the supervision of Geert Wiegertjes and Maria Forlenza in the Horizon 2020 project PARAFISHCONTROL. The aim of her work was to characterize polarized innate immune responses in carp with the emphasis on macrophages. During her research she has collaborated amongst others with her Japanese colleagues at Nihon University, and kept in close contact with her former supervisor Carla Piazzon. The most important results of this research can be found in this thesis. In the future she hopes to continue to learn and develop her skills as a researcher and contribute to a healthy, happy and green society for both humans and animals.

\section{List of publications}

Wentzel, A.S., Petit, J., van Veen, W.G., Fink, I.R., Scheer, M.H., Piazzon, M.C., Forlenza, M., Spaink, H.P. and Wiegertjes, G.F. Transcriptome sequencing supports a striking conservation of macrophage polarization in fish. Submitted for publication (2020).

Wentzel, A. S., Janssen, J., de Boer, V., van Veen, W. G., Forlenza, M. and Wiegertjes, G. F. Fish Macrophages Show Distinct Metabolic Signatures Upon Polarization. Frontiers in immunology 11, article 152 (2020). doi: 10.3389/fimmu.2020.00152.

Korytář, T., Wiegertjes, G. F., Zusková, E., Tomanová, A., Lisnerová, M., Patra, S., Sieranski, V., Šíma, R., Born-Torrijos, A., Wentzel, A. S., Blasco-Monleon, S., Yanes-Roca, C., Policar, T. and Holzer, A. S. The kinetics of cellular and humoral immune responses of common carp to presporogonic development of the myxozoan Sphaerospora molnari. Parasites \& vectors 12, article 208 (2019). doi: 10.1186/s13071-019-3462-3.

Katakura, F., Nishiya, K., Wentzel, A. S., Hino, E., Miyamae, J., Okano, M., Wiegertjes, G. F., and Moritomo, T. Paralogs of Common Carp Granulocyte Colony-Stimulating Factor (G-CSF) Have Different Functions Regarding Development, Trafficking and Activation of Neutrophils. Frontiers in immunology 10, article 255 (2019). doi: 10.3389/fimmu.2019.00255.

Piazzon, M. C., Wentzel, A. S., Wiegertjes, G. F. and Forlenza, M. Carp Il10a and Il10b exert identical biological activities in vitro, but are differentially regulated in vivo. Developmental and comparative immunology 67, p. 350-360 (2017). doi: 10.1016/j.dci.2016.08.016

Wiegertjes, G. F., Wentzel, A. S., Spaink, H. P., Elks, P. M. and Fink, I. R. Polarization of immune responses in fish: The 'macrophages first' point of view. Molecular immunology 69, 146-156. (2016). doi: 10.1016/j.molimm.2015.09.026

Piazzon, M. C., Wentzel, A. S., Tijhaar, E. J., Rakus, K. Ł., Vanderplasschen, A., Wiegertjes, G. F. and Forlenza, M. Cyprinid Herpesvirus 3 Il10 Inhibits Inflammatory Activities of Carp Macrophages and Promotes Proliferation of Igm+ B Cells and Memory T Cells in a Manner Similar to Carp Il10. Journal of immunology 195, 3694-3704 (2015). doi: 0.4049/jimmunol.1500926 


\section{Overview of completed training activities}

$\begin{array}{ll}\text { The basic package } & 3 \text { ECTS }\end{array}$

$\begin{array}{ll}\text { WIAS Introduction Day } & 2015\end{array}$

Ethics and Philosophy in Life Sciences 2016

Course on Essential Skills 2016

$\begin{array}{lr}\text { Disciplinary competences } & 16 \text { ECTS }\end{array}$

IMPRESS genomics and Bioinformatics Workshop (Leiden) 2015

Writing PhD research proposal 2016

Advanced Immunology training course 2016

(Eykman graduate school, Utrecht)

$\begin{array}{ll}\text { Research Training program for young researchers } & 2017\end{array}$

(Nihon University, Japan)

18th International Fish Immunology Workshop (Wageningen) 2017

$\begin{array}{ll}\text { Statistics for the Life Sciences } & 2017\end{array}$

$\begin{array}{ll}\text { Professional competences } & 4 \text { ECTS }\end{array}$

Project \& Time Management 2016

Teaching and supervising Thesis students $\quad 2016$

$\begin{array}{ll}\text { Scientific writing } & 2018\end{array}$

$\begin{array}{ll}\text { Presentation skills } & 4 \text { ECTS }\end{array}$

European Macrophage and Dendritic cell Society, Amsterdam (poster) 2016

International Society of Developmental and Comparative 2018

Immunology Conference, Santa Fe, US (oral presentation)

19th International Fish Immunology workshop, Wageningen (oral presentation) 2018

20th International Fish Immunology Workshop, Wageningen (oral presentation) 2019

3rd International Conference on Fish \& Shellfish Immunology, Las Palmas, Spain $\quad 2019$ 


\section{Teaching competences}

Human and Veterinary Immunology course

Cell Biology course (2x)

Cell Biology and Health course

Supervising major Master thesis $(2 \mathrm{x})$

Practicals supervision 17th, 18th and 20th International Fish Immunology Workshop

Education and Training total:

\section{ECTS}

2015

$2016 / 2017$

2019

2016-2018

2016-2019

Completion of the training activities is in fulfilment of the requirements for the education certificate of the Graduate School of the Wageningen Institute of Animal Sciences (WIAS). One ECTS equals a study load of 28 hours. 


\section{Acknowledgements}

After 4,5 years of pipetting, analysis and writing I am looking back at an amazing time and have learned so much, I do not know where to start thanking all of you. I am really grateful to all of you, and I hope I did not forget to mention this over the years. If that is the case, then many of the statements listed below will not come as a surprise.

Dear Geert and Maria, thank you for sharing your knowledge and experience, for your dedication and enthusiasm and for always making time to discuss and answer quick and not so quick questions. Dear colleagues, thank you for all the good times and gezelligheid, for nice chats over coffee, for all you have taught me inside and outside the lab. Lieve vrienden en familie, dankjulliewel voor alle support, de aanmoediging en het begrip als ik weer eens veel te weinig tijd voor jullie had. Lieve Nimphjes, dankjulliewel dat jullie al die tijd al naast me staan, en nu ook tijdens mijn defence. Daar ben ik heel erg blij mee. Lieve Wouter, dankjewel dat je er altijd voor me bent, dat je al mijn rariteiten accepteert en me altijd aanmoedigt. Ik ben trots op jou en op ons. Ik ben benieuwd wat de toekomst ons gaat brengen, op naar het volgende avontuur.

Thank you all for....

Sharing your knowledge and lab-skills with me - Never losing patience when explaining why we need a certain ionic strength again - Accepting me as one of the boys in the 'mancave' — Always making me laugh — Helping me out with Illustrator or InDesign hacks — Making me (and us) part of your amazing family - Sharing our beloved (and sometimes frustrating) macrophage cultures with me as my macrofagenbuddy - Helping me out with last minute FACS experiments - Meeting me for discussions at my home so I would not have to come to Zodiac - Inventing hilarious songs about me every day _ Amazing logo-design on call - Turning problems into 'challenges' and providing amazing opportunities _ Organizing 'fishy' evenings with nice food and presents — Getting fish for me from Carus when the sun was not even up - Providing me again and again with yet another transcriptome dataset — Amazing E-wing labuitjes and Sinterklaas party's - Bunny-sitting so I can go to conferences and on the odd weekend away/ week of holidays - Always picking out my books because I never know what to read next - Turning in well-written Msc theses that were a joy to read - 
Turning RijnBBQ’s into Gastro Grill Events - Pushing me to start programming and then patiently figuring out what I did wrong and avoiding me throwing my laptop out the window - Organizing your labjournal in such a way that I could find details if I needed them — Sending surprise packages that suddenly show up on my doorstep — Being digitally next to me during my defence — Discussing absolutely everything over way too many coffees - Hilarious times when shooting, editing and watching PhD movies - Bringing me to the other side of the world to share your recombinant-making skills — Making me laugh by racing around and making binkies on the couch - Weekly dinners followed by badly steering drivers - Giving me a talk at the fish workshop twice - Always knowing exactly where to find something in the lab after I could not find it Making trips to the supermarket to provide me with groceries during my cripple period - Video calling at the wee hours in the morning to wish me luck and see the rabbits - Making the effort to understand what it was again that I was studying and still listening even though you were lost long ago / Proberen te begrijpen wat ik ook alweer aan het onderzoeken was, en nog steeds luisteren ook al ben je de draad al lang kwijt — Relaxing and high speed forest rides on horseback — Feeding me the most crazy and delicious foods and sake — Writing the 'About the author' section in this thesis - Supporting me to the top of the island - Accepting that I am not coming/calling because I am working again Het brengen van een gigantische nieuwe koelkast naar mijn nieuwe huis in de auto waar hij eigenlijk net niet in past — Pushing me up the dike in a wheelchair to make sure I got out of the house every now and then - Designing and testing kilo's of primers on an correspondingly large number of sequences - Wheeking like little maniacs when I wake up or come home - Trying to teach me some of your 'not caring too much' - Sharing my knitting hobby and making everything that I did not find the time for — Being my wingwoman on the Il-4/13b1 recombinant journey — For trusting me to get abstracts and talks finished just in time - Teaching me how to make dry-ice bombs — Dancing in Las Palmas Autoruilen zodat ik toch nog ergens heen kon rijden — Racing me through US airports in a wheelchair — Seeing that our story is still more interesting than the one that just came out - Spending over a week full-time pipetting qPCRs because I really wanted to use my new M2 markers - Teaching me the essential wholeoffice-makeover skills — All the feedback on my work and my writing Reminding me that I am (not) crazy - Always making time in your crazy-full agenda to discuss experiments, check-out results, find something in the -80 , solve 
last minute issues - Regularly making modern art to cheer up our house Teaching me how to work with the inverted microscope - Showing me the ropes in Oropesa — Sharing my plant-addiction — Taking good care of our precious carp - Shared lunches and countless trips to the Appie - Teaching me the fingerspitzengefühl of the snufjes and slokjes that you need with FACS stainings and cytospins - Intense and exhausting climbing sessions with the \#Powerpof colleagues - Coming all the way from Utrecht to help me clean my house when I could not walk - Speed-ordering lab reagents and tracking lost orders Answering all my 'quick' questions - All the discussions that come together in this thesis - Helping to eat all the toddler-sized mutant zucchinis from the garden - Making our PhD weekends unforgettable - Considering every day a Friday afternoon when it comes to office music - Joining me in my race to the finish - Absolutely always being there for me, even though you are tired and busy yourself — The strangest discussions, jacuzzi-cultures and mountaingoats come to mind.... - Providing the renowned EZO helpdesk for all computer support - Bringing me to conferences to present my work and meet/wine/dine/ party with the fish people - Radler 0.0 breaks in the late afternoon before continuing work — And helping me finish in time, despite a dew hick-ups ;-) 
About the author

A 


\section{Colophon}

The research described in this thesis was financially supported by the European Commission under the 8th (H2020) Framework Program for Research and Technological Development of the European Union (PARAFISHCONTROL, Grant No. 634429).

Financial support from Wageningen University for printing this thesis is gratefully acknowledged.

Cover design by Annelieke Wentzel

Layout by Annelieke Wentzel

Printed by ProefschriftMaken || www.proefschriftmaken.nl 

